\title{
Tilted Fiber Bragg Grating Active Heater for Controlled and Localised Hyperthermia
}

\author{
by \\ SONDOS ABDULLAH ALQARNI
}

A Thesis Submitted to the Faculty of Graduate Studies and

Research in Partial Fulfillment of Requirements for the

Degree of Doctor of Philosophy

Carleton Institute for Electrical and Computer Engineering

Department of Electronics

Carleton University

Ottawa, Ontario, Canada

September 2018

(C) Copyright by Sondos Alqarni, 2018 


\begin{abstract}
We present data from localized heat inducement studies at both cellular and tissue levels along with a computational model built to predict the temperature increase and damage extent in tissues receiving hyperthermia treatment by a fiber-based active heater. This novel fiber-based active heater serves as a heat source and a temperature sensor. Five important insights are highlighted from this thesis work.

First, heat-induced controlled cell deaths were observed experimentally in the three cell lines with MCF-10A being more susceptible to heat compared to HEK 293 and MCF7 cells. Second, comparison between the phantom tissue and ex vivo experimental and computational results shows a lesion size of $5 \times 12 \mathrm{~mm}$ and $4.87 \times 11.6 \mathrm{~mm}$ in the phantom tissue and $7 \times 15 \mathrm{~mm}$ and $8.8 \times 14.3 \mathrm{~mm}$ in the ex vivo studies at pumping power of $1.8 \mathrm{~W}$ for 10 minutes respectively. Thus, this computational model is able to provide information about the heat transfer characteristics caused by the active heater in living biological tissue.

Third, under similar conditions of pumping power and heating time to that used in the ex vivo experiment, we found that the blood perfusion has a profound effect on the amount of induced heat at the active heater surface (or at the heat source). Because of the small dimension of lethal volume, heat dissipation by blood with a volumetric perfusion rate of $6.4 \times 10^{-3} \mathrm{Kg} / \mathrm{m}^{3} \mathrm{~s}$ in the liver tissues is very small.

Forth, in all the experimental and computational studies, hyperthermia position and damage extent can be controlled by the active heater through managing the temperature increase and the power supply during heating, thereby avoiding the transient effect of heat outside of the target volume. Thus, this hybrid simulation/active heater approach
\end{abstract}


may have the potential to provide reliable temperature increase information before and during a procedure in which controlled localized heating is required.

Finally, we show that when packaged, the fiber-based active heater does not suffer from a significant reduction in heating efficiency. The thermal stability of the heaters was shown to be stable at typical temperatures used in hyperthermia treatment. Hyperthermiainduced lesions by the packaged active heater were in order of $3.9 \times 14.5 \mathrm{~mm}$ and $3.787 \times 5.24 \mathrm{~mm}$. 


\section{Acknowledgments}

I am indebted first to God for giving me all the people whom support me during my $\mathrm{PhD}$ studies. I would like to express my gratitude to my supervisors, Prof. Jacques Albert and Prof. Christopher W. Smelser. I appreciate the freedom you have given me in conducting my experiments and provided me with the guidance and support whenever I needed help. Dr. Jacques, you have always made yourself available to me and to my research. He has provided me with a lot of fruitful comments and questions. His scientific vision and his way in reading reports along with his vast knowledge are very impressive, yet scary! I can literally say that I have always taken something from him each time we talked. Dr. Christopher, you were the first person I met in the group. Chris is someone you will instantly like; he is very kind and lively. I was very lucky that when I started my PhD degree in Fall 2014, you were teaching the adv. topics in electromagnetics course. It was very rich with the necessary information about guiding and coupling waves in general and particularly about the FBGs devices giving me a fast and easy start to my $\mathrm{PhD}$ research. He is always available to write letters for my sponsorship, to fix my writing, to discuss my experiments providing me with insightful comments. Each of you are very big-hearted and have given me a lot from your expertise and time over the last four years, so thank you so much. I express my special appreciation and thanks to my academic advisors Dr. Mohamad Najem and Dr. Yousef Abu-Nada at Saudi Arabian Cultural Bureau in Canada. During my grad studies, they were always keeping in touch with me and had helped me with all the matters I faced. Besides keeping track of my academic progress, they have provided me with unlimited suggestions, supports and advices, "thank you so much". 
I particularly wish to thank Dr. William Willmore, Professor and Director of the institute of Biochemistry, Department of Biology at Carleton University, for collaborating and sharing his knowledge with us and for having welcomed me at his lab. He provided me with all the materials, which I needed, to complete my studies on the cells. Great thanks go to Dr. Martin Bernier, Associate Professor, Department of Physics, Engineering Physics and Optics at Université Laval, for collaborating with us in the thermal studies part of this thesis work. He invested his time in our work, provided us with valuable feedback and fabricated the femtosecond devices for us.

I thank my thesis proposal defence chair Dr. Tom Smy, and committee member Dr. Steven McGarry for all their contributions of ideas, advices, and time to make my thesis proposal defense a productive meeting. Certainly, I am quite appreciative of my thesis defence committee members; Dr. Luc Lévesque, Dr. Anatoli Lanoul, Dr. Rony Amaya, and Dr. Steven McGarry, for generously putting their time and effort to read my thesis and provided me with constructive comments that shaped the final version of my thesis.

Many thanks are required to Dr. Saud al-Lehiani and Dr. Faiz Alghorabie, Professors at Umm Al-Qura University in KSA, and Dr. Young Ki Yoon, Dr. Lan Wei, and Dr. Andrew Brzezinski, Assistant Professors at Waterloo University in Canada, for taking the time to fill out the recommendation form on my behalf.

I would like to thank Albane Laronche and Hubert Jean-Ruel. They helped me to start my experiments. I had never worked with most of the devices in the lab. Without them I would have spent three hundred years in a cave at the library wondering why I could not see the Bragg resonance! 
I acknowledge the financial support which I have received from King Abdullah Scholarship since 2010.

I would like to offer my special thanks to my family and to my family-in-lows, especially my parents, Abdullah and Aisha for always believing in me and for their continuous love.

Finally, my deepest gratitude is to my husband and children. Ahmed, I am very lucky to be married to; I love you. He has been my rock and my inspiration. My $\mathrm{PhD}$ journey was not easy without his encouragement and support I would not be able to complete it. My son, Majody, and my baby girl, Rose, are the joy of my life. They have continually given me the most wonderful time and breaks from my studies and anxiety, thanks my little ones in helping mommy to get her degree. 


\section{Table of Contents}

List of Tables ................................................................................................ List of Illustrations ...................................................................................

1 Introduction............................................................................................

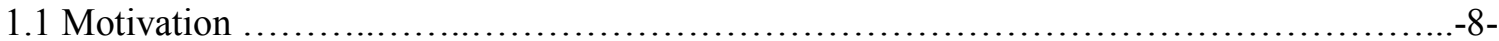

1. 1. 1 Real-time Temperature Monitoring Systems ....................................

1. 1. 2 Achievement of Localized and Control therapy, researchers' goal ......................-9-

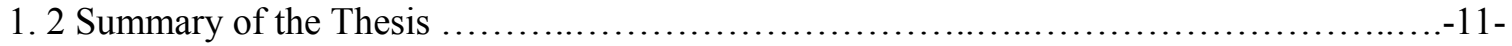

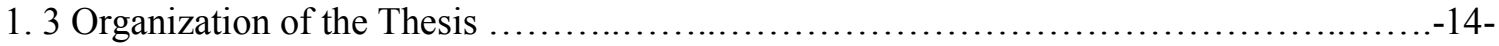

2 Local Hyperthermia Therapy......................................................... 15-

2. 1 Introduction to Local Hyperthermia (LHT) ........................................

2. 1. 1 Local Hyperthermia Based on Indirect Heating ..................................

2. 1. 1. a Microwave heating...........................................................

2. 1. 1. b Radio-frequency hyperthermia .................................................

2. 1. 2 Local Hyperthermia Based on Direct Heating.................................... $21-$

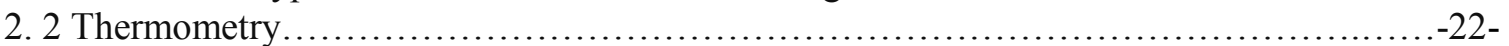

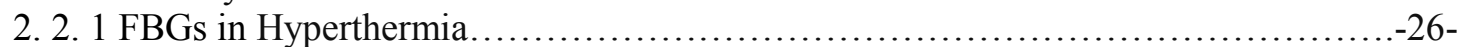

2. 2. 1 a FBGs problems......................................................... $28-$

2. $2.1 \mathrm{~b}$ Role of FBG in the development of hyperthermia............................

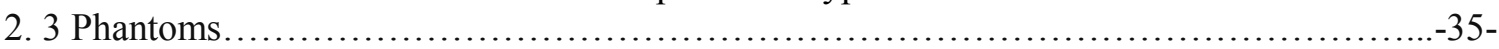

3 Grating-Based Fiber Optic Sensors..................................................42-

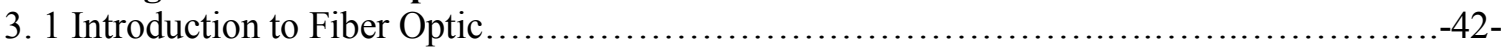

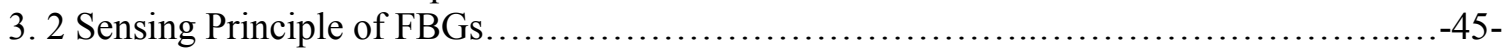

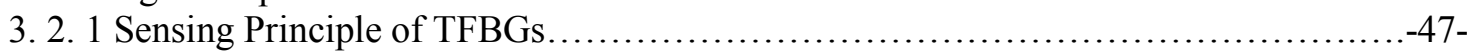

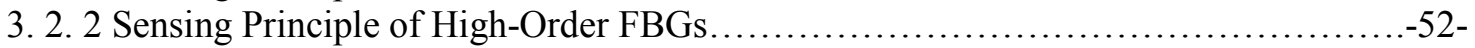

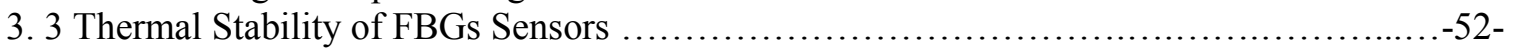

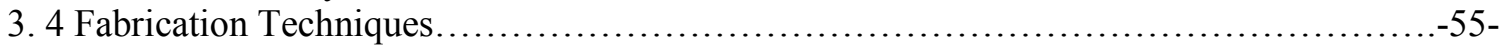

3. 4. 1 Holographic Method............................................................

3. 4. 2 Phase Mask Method.......................................................... $57-$

3. 4. 2. a High Order FBGs.....................................................

3. 4. 2. b TFBGs.................................................................

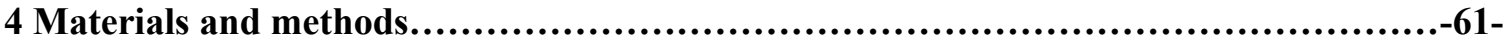

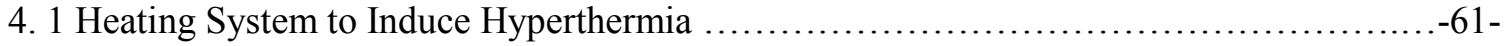

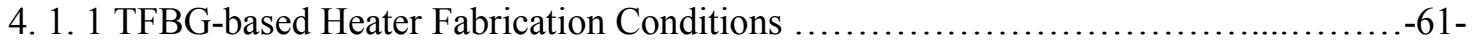

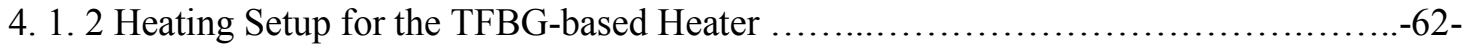

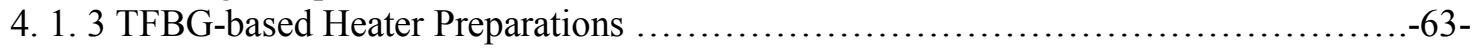

4. 1. 3. a Coating Materials Evaluation for the TFBG-based Heater....................-64-

4. 1. 3. b Packaging the TFBG-based Heater ..................................... 66 -

4. 1. 4 Heated Target Materials ...........................................................

4. 1. 4. a Cell Lines and Cell Cultures ............................................ 67 -

4. 1. 4. b Tissue-Like Materials ................................................... $69-$

4. 1. 4. b. i Gelatine-based Phantom .......................................

4. 1. 4. b. ii Egg White-based Phantom ...................................

4. 1. 5 Ex Vivo Porcine Liver....................................................

4. 2 Annealing System to Evaluate the Gratings Thermal Stability.........................

4. 2. 1 Ultrafast FBGs Fabrication Conditions.......................................

4. 2. 2 Ultrafast FBGs Annealing Setup................................................ 
5 Results and Discussion ...............................................................

5. 1 Optical Properties of the TFBG-based Heater .......................................

5. 2 In Vitro Hyperthermia of HEK293, MCF-10A, and MCF7 Cell Lines ........................-80-

5. 3 Hyperthermia of Egg White Phantom, Gelatine Phantom and Ex Vivo Hyperthermia of

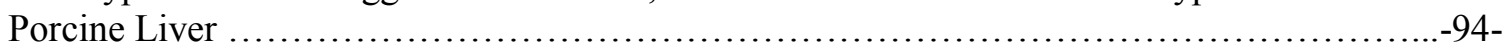

5. 3. 1 Hyperthermia-induced Lesion in Egg White Phantom by the Packaged TFBG-based

Active Heater................................................................ $97-$

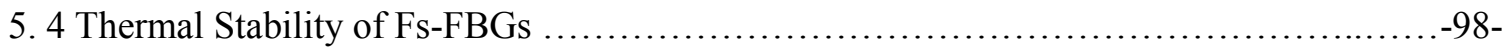

6 Mathematical Modelling................................................................-102-

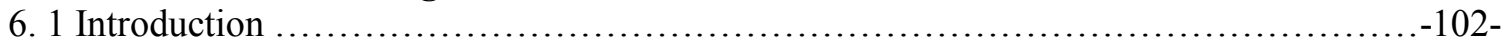

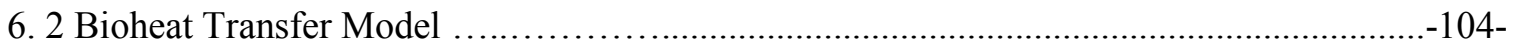

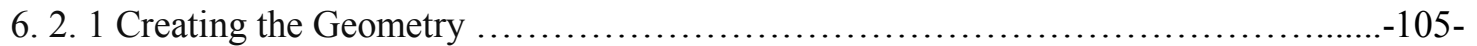

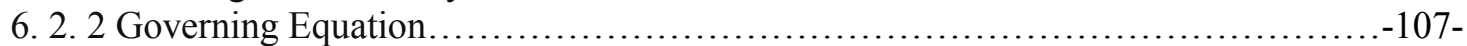

6. 2. 2. a. Prior to Pennes' Work .................................................... $108-$

6. 2. 2. b Pennes' Bioheat Transfer Equation .......................................

6. 2. 2. c Pennes' Bioheat Transfer Equation in Hyperthermia.............................

6. 2. 2. d Forming the Bioheat Transfer Equation for Our Work.........................

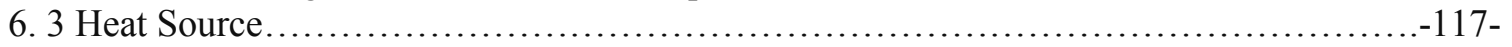

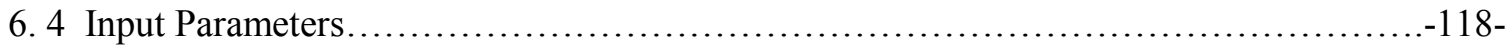

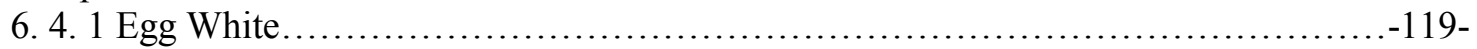

7 Results of Mathematical Modelling...................................................126-

7. 1 Computational Results of Hyperthermia in Egg White Phantom (model validation).......-126-

7. 2 Computational Results of Hyperthermia in Liver Tissues .........................................-131-

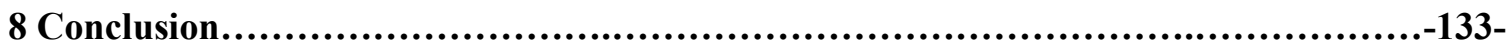

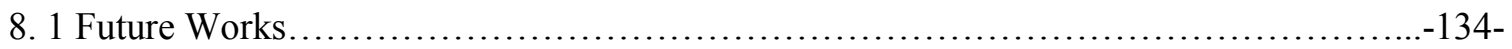

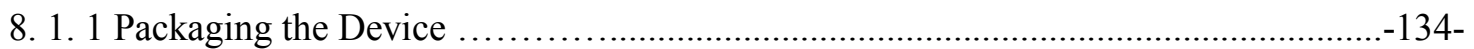

8. 1. 2 Design Multiple Localized Heating Points.......................................................... -135-

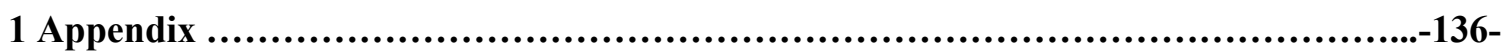

1. 1 Alternative Bioheat Transfer Models ......................................................

1. 1. 1 Specific Absorption Rate (SAR) .......................................... 137-

1. 2. Heat Transfer by Conduction...............................................

2 Appendix.............................................................................-141-

2. 1 Journal and peer reviewed conference publications ......................................

2. 1. 1 Conference publications ................................................ 141 -

2. 1.2 Conference presentations ............................................ 141 -

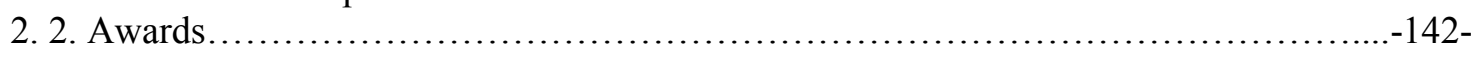

Bibliography....................................................................... 


\section{List of Tables}

Table 1.1. Treatment conditions of two types of hyperthermia; local \& regional, and systemic (the whole body). The local \& regional are selective treatments used to cause direct damage to a tumor tissue, while the systemic treatments are not selective and used to increase the tumor sensitivity

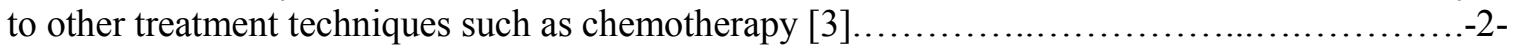

Table 1.2. Biological effects corresponding to each hyperthermic temperature [1] ....................-2-

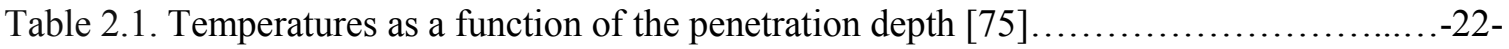

Table 2.2. The Available thermocouples in the market according to the ANSI classification

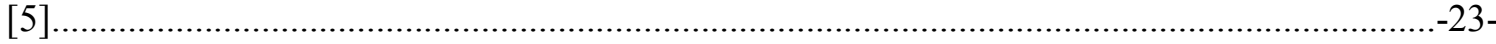

Table 2.3. Diffrecnt type of thermocouples with their sensing characteristics [72]............-24-

Table 2.4. Materials' percentage used to synthesis heterogeneous phantoms [119].............-39-

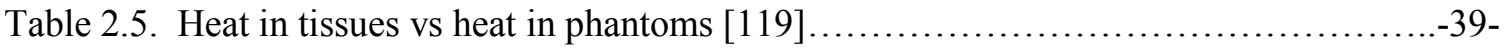

Table 2.6. Approximation of heat capacity of pig, cow, and human blood vs egg white

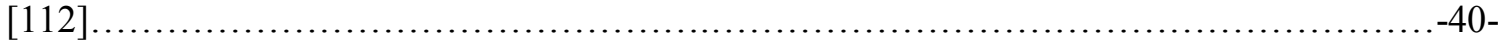

Table 6.1. Geometries and subdomains created in the model............................

Table 6. 2. Heat transport modes in different organs in a living body $\ldots \ldots \ldots \ldots \ldots \ldots \ldots \ldots . \ldots \ldots \ldots \ldots$ - $112-$

Table 6. 3. Thermal properties of the utilized materials in the simulation....................-125- 


\section{List of Illustrations}

Figure 1.1. Difference between performing hyperthermia in combination with radiotherapy and

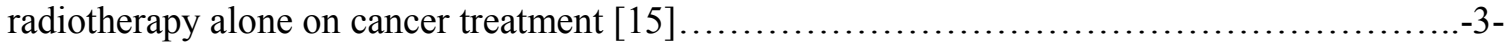

Figure 1.2. Schematic diagram of tumor tissues; tumor tissues are abnormal, meaning that they

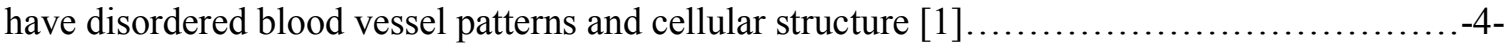

Figure 1.3. An example of one of the treatment' goal in oncology, using nanoparticles to achieve more targeted treatments (by absorbing more energy and hence causing a larger temperature increase ) and less harmful overall to the body. Temperature increase at different depths with and without the nanoparticle (a) simulated results, and (b) experimental results [53] ...............

Figure 2.1. An applicator shape of radiofrequency ablation hyperthermia $[1] \ldots \ldots \ldots \ldots \ldots \ldots-20-$

Figure 2.2. An example of applicator used to perform local hyperthermia based on direct heating

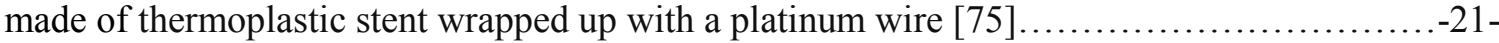

Figure 2.3. Schematic diagram of fluorescent structure, and temperature as a function of the

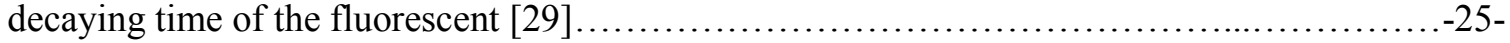

Figure 2.4. Schematic diagram of the applicator used in vivo to measure the temperature increase the kidneys and livers of rabbits during hyperthermia [89]. -27-

Figure 2.5. Compared FBGs (circles) output measurements with those of the Fluor optic probe

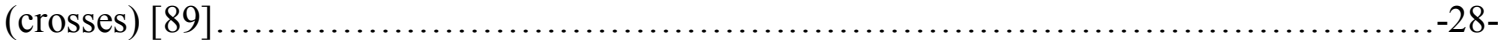

Figure 2.6. Schematic diagram of FBGs thermometry's and their positions in a liver phantom

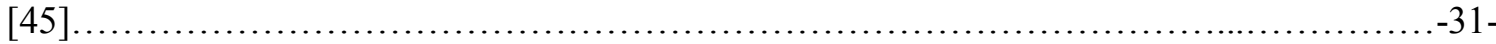

Figure 2.7. Comparison between the theoretical (on the left) and experimental (on the right), temperature measurement at different distance from the applicator, continuous line $(5 \mathrm{~mm})$, dashed line $(10 \mathrm{~mm})$, and dashed-dotted line $(15 \mathrm{~mm})$ [93]

Figure 2.8. Temperature distribution along longitudinal axis as a function of time for three different RFTA experiments with applying the same power in order of $20 \mathrm{~W}$ RF: the temperature disruption along the applicator is not a function of time in the liver due to difference in the liver

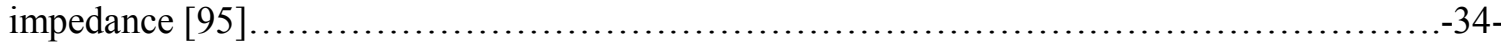

Figure 2.9. Image of TMTC phantom cross section along the RF electrode to simulate the ablation effect on soft tissues such as liver (A) and with the temperature distributions (B) [115] $-36-$

Figure 2.10. Various aluminum and graphite concentrations in polyester resin mixture produce phantoms with different electrical properties; each mixture can be used to mimic the properties of

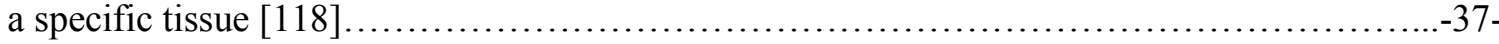

Figure 2.11. Long-time stability up to nine weeks; phantom conductivity after one week of the phantom preparation (solid lines); and after nine weeks of the phantom preparation (dashed lines) [107] $-38-$ 
Figure 2.12. A heterogeneous phantom developed to simulate the dialectical and thermal properties of a human thigh with tumor tissues by Yuan et al [119] ..........................-38-

Figure 2.13. Effective temperature needed to cause irreversible visible lesion [120]...........-41-

Figure 3.1. An index modulation inside SMF-28 optical fiber, and the sensing principal of FBGs based temperature sensors with transmissive or reflective detection options .................. 45 -

Figure 3.2. Bragg guided mode and cladding mode resonances in the transmission spectrum of the TFBG because of the presence of the inclination angle.................................... 48 -

Figure 3.3. An example of the transmission, reflection, and radiation spectra of second-order

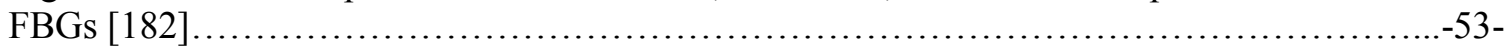

Figure 3.4. The draw-tower setup; the gratings are written at the same time as the fiber get

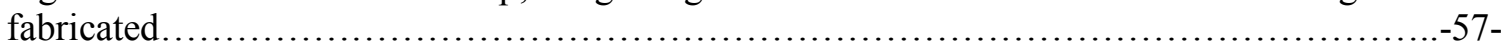

Figure 3.5. The phase mask technique to fabricate TFBGs............................... 59 -

Figure 4.1. Transmission spectrum of the TFBG-based active heater........................62-

Figure 4.2. Schematic diagram of the heating setup................................. $63-$

Figure 4.3. Transmission spectrum of TFBG before and after cleaning taken in the oven......-64-

Figure 4.4. Effect of different type of coatings on the heating efficiency of TFBG-based active heaters as a function of the increase in the pump power in increment of $200 \mathrm{~mW}$ per minute. $-65-$

Figure 4.5. Bragg resonance shift in correspondence to the coated layer temperature; reflection spectrum of the cobalt blue coated TFBG-based active heater. $-66-$

Figure 4.6. Cells grown in $6 \mathrm{~cm}$ plates on top of the active heaters; (a \& b) HEK 293 cells on top of a bare active heater: (a) for just visualisation: we can not see the cells grown on top of the active heater due to the black color of the coating layer; (c) MCF-10A and (d) MCF7 cells grown on top of the coated active heater. (e) HEK 293 (f) MCF-10A and (g) MCF7 cells viability after exposing to heat with maximum temperatures of $54{ }^{\circ} \mathrm{C}$ for 5 minutes; after the treatment we used

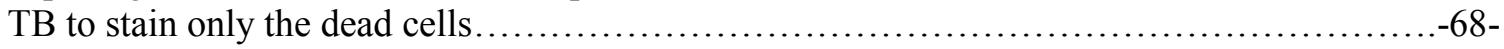

Figure 4.7. TFBG-based active heater enclosed in the needle inside the jelly.................-70-

Figure 4.8. The calculated effect of heating at pump power of $1.8 \mathrm{~W}$ for 10 minutes in the hen egg white, human and porcine liver without considering the blood perfusion effect................-71-

Figure 4.9. Permanent colour changes of the thermochromic ink vs temperatures............-72-

Figure 4.10 Hyperthermia under the same pump power in order of $1.8 \mathrm{~W}$ for 5 - and 10- minutes; top (upper panel) the active heaters were sandwiched between the liver tissues; side (lower panel) lesions number 1 and 2 result from continuous heating for 5 minutes, lesions number 3 and 4 result from continuous heating for 10 minutes. $-72-$ 
Figure 4.11. Exposure conditions used to write the FBGs through the polyimide coating.......-73-

Figure 4.12. The growth behavior of first and third-order gratings........................

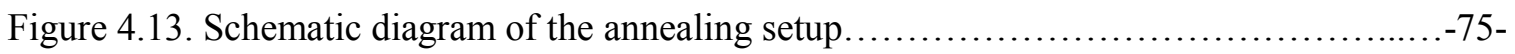

Figure 5.1. A comparison between pumping at single wavelength and over a range of

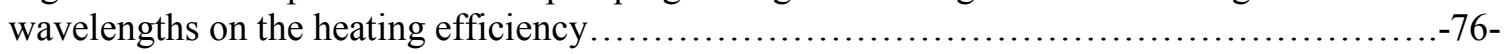

Figure 5.2. Bragg wavelength shift as a function of the pump power increase (left), the corresponding temperature change (right) ......................................................

Figure 5.3. (a) Transmission spectra of TFBGs with different strengths $(6,14, \& 16.7 \mathrm{~dB})$ with their transmission spectra after applying the absorptive coating layer; (b) temperature increase at a fixed power of $1.4 \mathrm{~W}$ as a function of increasing grating strength measured in water. $-78-$

Figure 5.4. Active heater written under the same conditions: (a) reflection spectra of 10 active heaters; (b) the resulting temperature increase .......................................

Figure 5.5. (a \& b) HEK 293 cells viability after exposing to heat with temperatures of 53 for 5 minute. After the treatment we used TB to stain only the dead cells ........................ $81-$

Figure 5.6. MCF7 cells viability as a function of pump power and time................-83 to $86-$

Figure 5.7. MCF-10A cells viability as a function of pump power and time.............-87 to $90-$

Figure 5.8. HEK 293 cells viability as a function of pump power and time..................-91-

Figure 5.9. (a \& b) Survival cells rate as a function of power and time; MCF-10A and MCF7 cells viabilities were normalized to cells grown under the same condition without exposure to

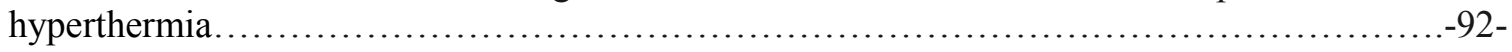

Figure 5.10. The degree of the thermal damage as a function of heating time at a fixed power, and qualitative assessment of temperature gradients in correspondence to the color change of the ink.; 3 temperature regions represented by the symbol $(\times):(\times 1) 139^{\circ} \mathrm{C},(\times 2) 70{ }^{\circ} \mathrm{C}$, and $(\times 3) 50$ ${ }^{\circ} \mathrm{C}$. $-94-$

Figure 5.11. The degree of the thermal damage as a function of heating time at a fixed power in ex vivo porcine liver. $-95-$

Figure 5.12. TFBG-based active heater enclosed in the needle inside the fresh hen egg white

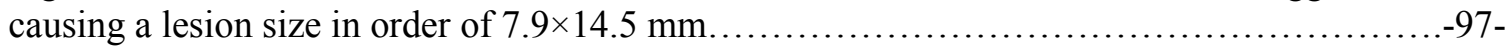

Figure 5.13. Lesion size in order of $7.583 \times 5.24 \mathrm{~mm}$ and the increase in temperature measured by the packaged TFBG-based active heater and the temperature increase in correspondence to Bragg

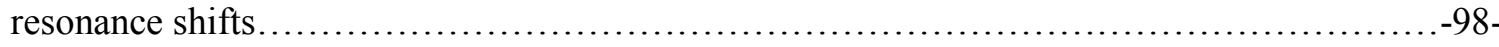


Figure 5.14. Transmission spectrum at room temperature of the (a) first-order grating before and after the regeneration process, and (b) third-order grating before and after annealing............ 99.

Figure 5.15. Comparison of $1^{\text {st }}$ and $3^{\text {rd }}$ order grating's normalized index change during the short

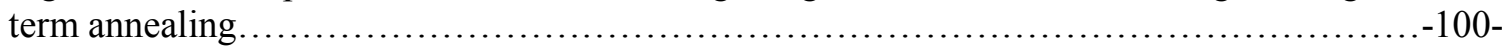

Figure 5.16. Comparison of $1^{\text {st }}$ (blue) and $3^{\text {rd }}$ (green) order grating's normalized index changes

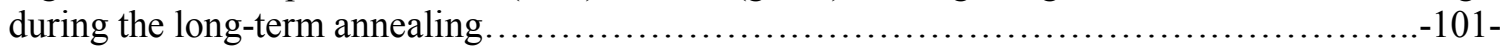

Figure. 6.1. Geometrical shape of the simulated material...............................-104-

Figure. 6.2. Types of mesh in COMSOL: (a) Structured mesh can be used for simple geometries. Also, the number of nodes and elements has to be equal: (b) Unstructured mesh gives high

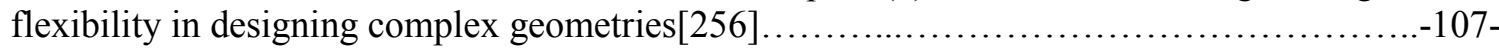

Figure 6.3. Temperature gradients distribution from the surface "skin" down to the axis of the

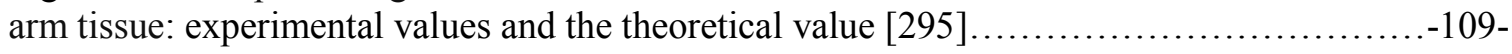

Figure 6.4. Schematic diagram of the egg white denaturation process $[230] \ldots \ldots \ldots \ldots \ldots \ldots . . . \ldots \ldots-120-$

Figure 6.5. Egg white properties as a function of Temperature........................... $123-$

Figure 6.6. Heat flux of the egg white as a function of temperature $[226] \ldots \ldots \ldots \ldots \ldots \ldots \ldots . . \ldots \ldots \ldots . . .123-$

Figure 6.7 Density of the egg white as a function of temperature $[236] \ldots \ldots \ldots \ldots \ldots \ldots \ldots . .124-$

Figure 6.8 . Thermal conductivity of a protein as function of temperature $[260] \ldots \ldots \ldots \ldots \ldots . . .124-$

Figure 7.1. The experimental and calculated effect of heating at pump power of $1 \mathrm{~W}$ for 5 minutes in egg white: (a) comparison of the temperature variations at different locations between the simulation (dotted curves) and the experiment (solid curves) results.................... 127-

Figure 7.2. The experimental and calculated effect of heating at pump power of $1.2 \mathrm{~W}$ for 5 minutes in egg white: (a) comparison of the temperature variations at different locations between the simulation (dotted curves) and the experiment (solid curves) results................... 128 -

Figure 7.3. The experimental (a \&b) and calculated (c \&d) effect of heating at pump power of 1.8 $\mathrm{W}$ for 10 minutes in egg white: the dimensions of the obtained lesion; based on the experiment results, we consider $55^{\circ} \mathrm{C}$ to be the transitional denatured temperature (represented by the blue

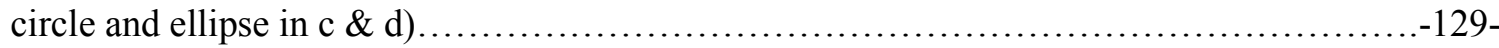

Figure 7.4. The experimental (a \&b) and calculated (c \&d) effect of heating at pump power of 1.8

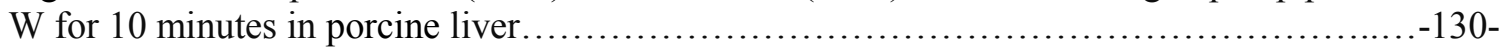

Figure 7.5. Temperature increases along the gratings' surface as a function of time...........-131-

Figure 7.6. Simulate the effect of blood perfusion on the heat-induced damage caused by the active heater in porcine and human livers. $-132-$ 


\section{Chapter 1: Introduction}

The method of treating illness with heat dates back to the $4^{\text {th }}$ century. Both the Greeks and the Romans stated the correlation between controlling one's body temperature and human health [1-5]. Thermal therapy or hyperthermia has long been considered as an alternative to conventional treatments for certain diseases owing to the basic equipment utilized for heat generation or temperature measures, lack of knowledge about the effect of hyperthermia, as well as no qualification requirements imposed on the person who performs the treatment. However, spread of fatal diseases along with increases in health care costs have prompted a need to explore new treatment techniques, one of which is hyperthermia.

Currently, hyperthermia has been applied to treat a number of diseases and conditions such as vision correction [6], local pain [7], prostatic hyperplasia [8, 9], cosmesis such as dermal tightening $[10,11]$, stabilization of skeletal joins $[12,13]$, and cancerous diseases [1-5,14-17]. Developments in "local hyperthermia therapy" combined with imagingguidance is the rationale behind the transformation of the modern thermotherapy.

Hyperthermia in oncology, according to the National Institutes of Health (NIH), is a therapeutic approach that has been used to treat various types of cancer or prevent and limit the further growth of tumor cells through heating the biological tissues of a tumor without damaging the normal tissues $[1-5,14-16,18]$. This procedure consists of elevating the temperature of tumor-loaded tissue from a few to as much as a hundred degrees. The thermal dosage required to perform the treatment depends on the type of the source applied to deliver hyperthermia and the depth, location, and stage of the cancer (see Table 
1.1). Accordingly, the thermal dose regimes have been grouped as follows: i) medium-to high- temperature hyperthermia $\left(39.5{ }^{\circ} \mathrm{C}-41.8{ }^{\circ} \mathrm{C}\right)$; ii) traditional hyperthermia $\left(41.5^{\circ} \mathrm{C}\right.$ $45{ }^{\circ} \mathrm{C}$ ); iii) thermoablation (over $60{ }^{\circ} \mathrm{C}$ ); and iv) thermal gasification (over $200{ }^{\circ} \mathrm{C}$ ). Table 1.2 indicates the biological effect corresponding to each temperature.

\begin{tabular}{|c|c|c|}
\hline \multirow[b]{2}{*}{ Technical parameters } & \multicolumn{2}{|l|}{ Hyperthermia } \\
\hline & Local/regional & Systemic \\
\hline Time to reach the stationer state & $5-50 \mathrm{~min}$ & $30-180 \mathrm{~min}$ \\
\hline Complete treatment time & $30-90 \mathrm{~min}$ & $120-720 \mathrm{~min}$ \\
\hline Maximal safe temperature (in the tumor) & Not limited (incl. ablation) & $42^{\circ} \mathrm{C}$ \\
\hline $\begin{array}{l}\text { Systemic safety control (temperature, } \\
\text { pulse rate, p02, ECG, } \\
\text { blood pressure, electrolytes, etc.) }\end{array}$ & Not relevant & Necessary \\
\hline Local safety control & Necessary & Not relevant \\
\hline
\end{tabular}

Table 1.1. Treatment conditions of two types of hyperthermia; local \& regional, and systemic (the whole body). The local \& regional are selective treatments used to cause direct damage to a tumor tissue, while the systemic treatments are not selective and used to increase the tumor sensitivity to other treatment techniques such as chemotherapy [3].

$\begin{array}{ll}\text { Temperature }\left({ }^{\circ} \mathrm{C}\right) & \text { Heat Effect } \\ 43-45 & \begin{array}{l}\text { Time dependency and reversibility, increase of tissue perfusion and permeability, rise } \\ \text { of drug concentration and/or activation, improvement of drug metabolism rate, } \\ \text { enhancement of cell cycle effect, rise of } \mathrm{pH}, \text { strengthening of the sensitivity of tissues } \\ \text { to radiotherapy } \\ \text { Reduction of enzymatic activity }\end{array} \\ & \text { Denaturization and coagulation of proteins } \\ >47 & \text { Desiccation and dehydration of tissues } \\ 50-60 & \text { Water molecules in tissues start boiling and evaporate, resulting in nonreversible } \\ 90-100 & \text { damages } \\ >100 & \text { Carbonization of tissues } \\ >150 & \text { Vaporization of tissues } \\ >300 & \text { Tissue begins to bum with appearance of firelight } \\ >500 & \text { Data courtesy of Hans Oaw Rolfsnes, Dan Neumann, and Prof. Paul Stauffer, University of California, San Francisco. }\end{array}$

Table 1.2. Biological effects corresponding to each hyperthermic temperature [1]. 
As a further advantage, hyperthermia can be utilized as an adjunct therapy to other conventional cancer therapy techniques including surgery, radiotherapy, and chemotherapy $[1,2,5,19-22]$. The benefit of this combined therapy approach is constructed from various factors including sensitized radiotherapy and chemotherapy along with reduction of toxins and some side effects. As well, if applied prior to surgery, hyperthermia reduces the reoccurrence of tumors and increases the cure rates [14]. Figure 1.1 shows an example of one of the pilot trials conducted to illustrate the effect of utilizing hyperthermia in combination with radiotherapy [15].

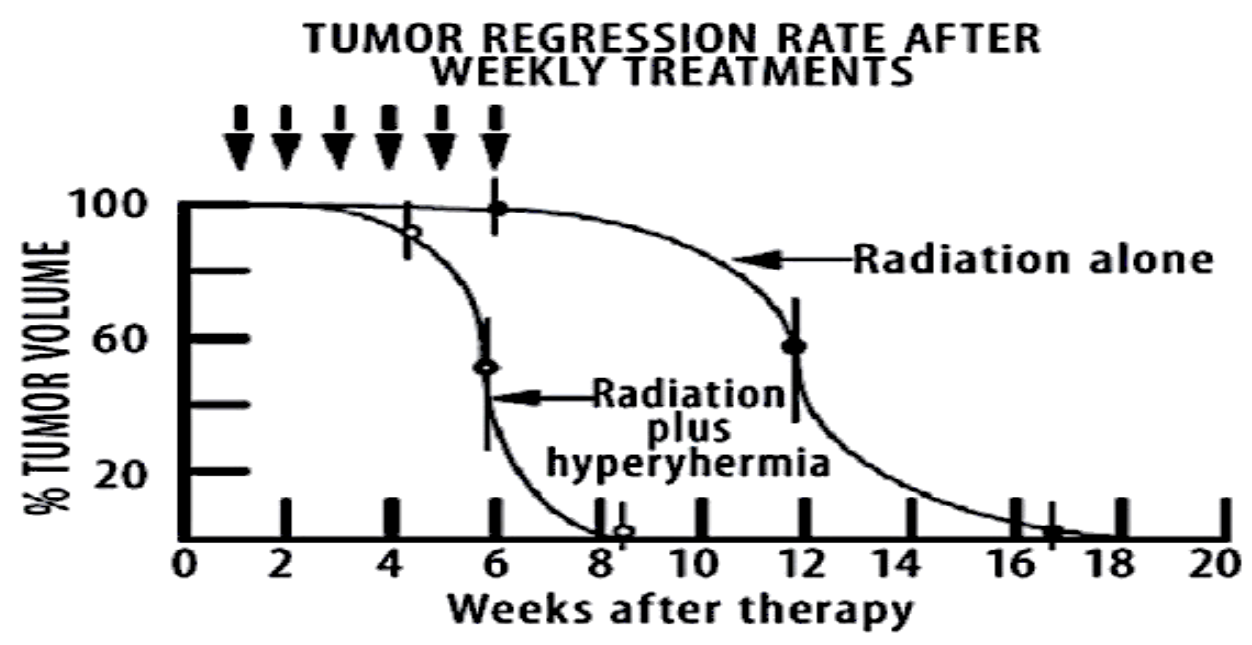

Figure 1.1. Difference between performing hyperthermia in combination with radiotherapy and radiotherapy alone on cancer treatment [15].

The structure of tumor tissues differs from that of normal tissues (Figure 1.2) [1, 3-5, 15, 16]. Tumor tissues are abnormal, meaning that they have disordered blood vessel patterns and cellular structure. This results in numerous abnormal biological activities including insufficient regional distribution of blood vessels which in turn lead to vessel walls with weak structure and basement membrane with no elasticity; cells with overflow blood or even no basal lamina; and weak vascular receptor causing a lack in the cells' ability to control their temperatures. The anomalous properties of tumor tissues produce a hostile 
or non-functional cellular environment comprised of higher temperatures than that of normal tissues, low blood flow, low $\mathrm{pH}$, and lack of oxygen.

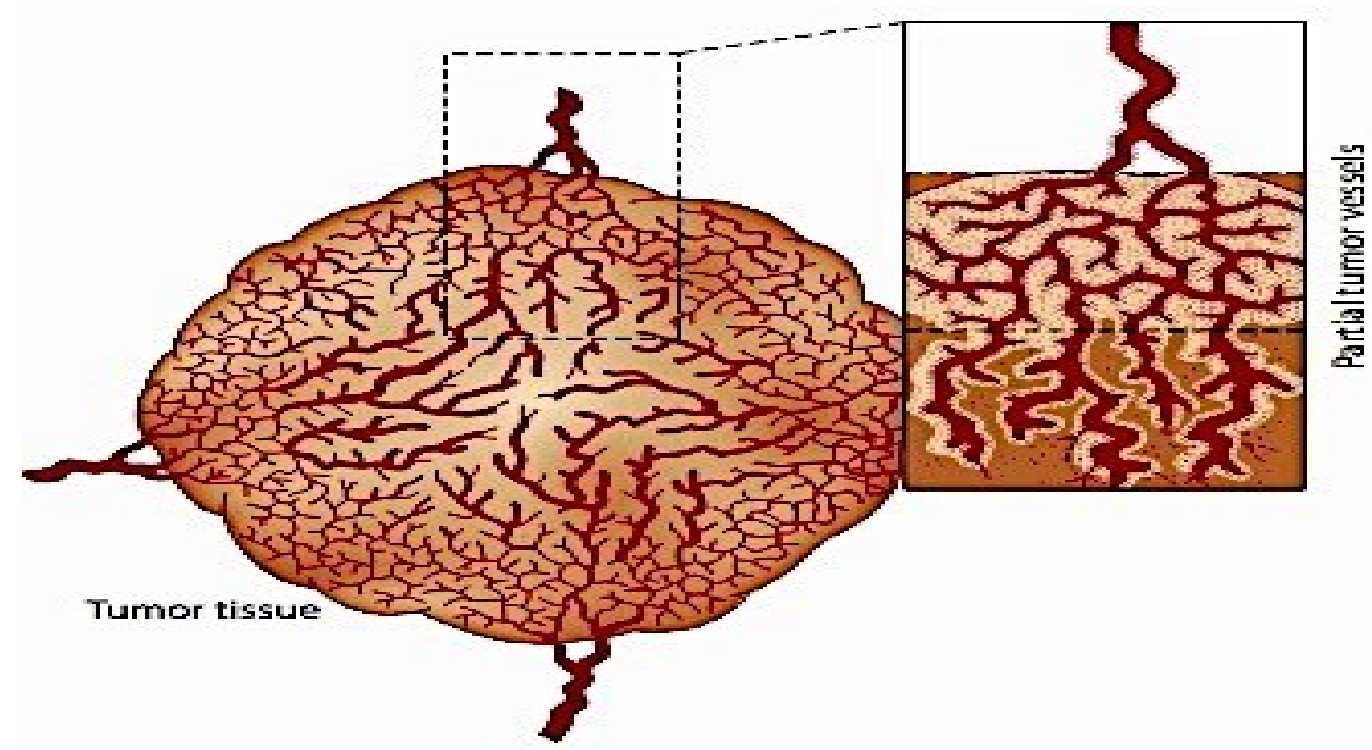

Figure 1.2. Schematic diagram of tumor tissues; tumor tissues are abnormal, meaning that they have disordered blood vessel patterns and cellular structure [1].

Thus, when heat is applied to a specific volume, selective cell damage can be obtained as long as the temperature of the applied therapy is less than $43{ }^{\circ} \mathrm{C}[1,3-5,15,18]$. Besides the ability to develop heat tolerance, normal tissues are able to dissipate heat smoothly. Since normal tissues have a normal and large amount of blood flowing through their cells, under an abnormal rise in the cells' temperature the blood vessels expand to increase the blood flow; thus, the heat is taken away. On the other hand, the blood circulation in tumor cells is very poor with low blood velocity; hence, the heat accumulates inside the cells during hyperthermia. Consequently, the temperature of tumor tissues is approximately $3-7{ }^{0} \mathrm{C}$ higher than that of normal tissues undergoing hyperthermia with the same conditions. Hence, more selective destruction can be achieved. 
Although hyperthermia is an artificial approach to increase temperature in a particular volume, the biological nature of the working principle behind hyperthermia cancer treatment over other cancer treatment techniques justifies its extensive interest and rapid development [1-5]. In addition, hyperthermia cancer treatment is not just an effective approach to remove malignant growth, as it also increases the tolerance of patients through, for example, simulating cancer immunity by helping the formation of heat shock proteins outside of cell membranes [23].

Based on such features, hyperthermia cancer treatment has transitioned from an alternative treatment to a treatment option for some cancerous diseases in many countries including Japan and China, where it is very developed; Germany; and Holland [1-5, 24]. Currently, hyperthermia is considered the fifth therapeutic technique for cancer following the techniques of surgery, chemotherapy, radiotherapy, and biotherapy.

Inducement of heat within living organs can be achieved through various sources including microwave, radiofrequency, endogenetic field, and light beam [1, 3-5, 25]. However, whether the techniques are already available clinically, including radiofrequency hyperthermia and laser-induced interstitial thermotherapy, or whether the techniques are still in the development stages such as microwave hyperthermia and magnetic induction hyperthermia, these techniques share the same problematic issues in controlling and monitoring the temperature distribution in the treated volume. This difficulty may result in the presence of some residual tumor, and hence inadequate therapy. 
A number of factors have caused this bottleneck. First, the non-homogenous temperature distribution is caused by biological activities such as the metabolic rate effect and blood perfusion, and by the tissue's physical properties including conductive or convective heat transformation and different electrical tissue properties [5, 26-29]. These factors affect the reliability of temperature readings from point sensors. For sufficient treatment of a specific volume without damage, the neighbouring healthy tissue requires the following: (i) applying disrupted localized sources to evoke the homogeneity of heat rise, and (ii) a reading of the temperature disruption within the tumor rather than temperature readings over a small volume. Accruing the temperature disruption within the target zone must be between separation points of approximately 0.5 to $1.5 \mathrm{~cm}$ to overcome the non-uniform heat flow resulting from the variation in the physical properties of cells $[20,30]$. These separation points should also have implications on the distance between antennas or electrodes used as a heat source to obtain sufficient heating. Such large number of applicators in close distance are very invasive, risky, and not clinically possible.

Second, there are technical issues with temperature sensors [26-29]. For example, there is uncertainty in the temperature measures obtained from the utilized thermometers due to black pigments in the coating of floroptic sensors and the metal parts in thermocouples. A more significant problem is associated with using the thermocouple as no temperature measurements can be obtained when the heating source is in the on mode. As a consequence, developments and improvements of thermometric techniques have been grouped into two main categories: invasive, including thermocouples, thermistors, and fiber-optic sensors (FOS) [31]; and non-invasive, such as photoacoustic (PA) sensors [32], CT-, MR- [33, 34], and ultrasound-based thermometers [35, 36]. 
The invasive thermometry technique, also known as intrusive thermometry, requires insertion of the sensors into the tissues of the living body after enclosing them into a catheter or needle [26-29]. In principle, a number of complications should be raised since the method of implementing these thermometers is invasive. Each of the available invasive-sensors has advantages and disadvantages with its use based on type of energy source, diameter of the probe, required number of sensors per probe, sensing object size, and location [37].

Currently, Fiber Bragg Grating (FBG) sensors are a focus of research both academically and within the industry. FBG sensors are considered ideal for in vivo sensing on account of the following [5, 24, 26-29]:

(1) Their intrinsic nature simplifies signal processing.

(2) Biocompatibility.

(3) Immunity to electromagnetic interference and small size (far less smaller than the required size of the ideal thermometry in clinical practice which is less than $2 \mathrm{~mm}$ ).

(4) Capability of multiplexing sensing with no need to increase the probe size to provide appropriate temporal and spatial measurements.

(5) High accuracy and real time feedback (higher than the required accuracy which is less than $0.2{ }^{0} \mathrm{C}$ and faster than the required response time which is less than 4 seconds). (6) Low-cost with the ability to be fabricated for mass-production. 


\section{1 Motivation:}

Performing localized treatment for many fatal diseases has become the foremost goal in the medical field as more efficient disease treatment reduces the risk of further short- or long-term complications. Based on the literature review illustrated in chapters 1, 2, and 3 that thermal therapy in oncology has the ability to provide selective localized treatment. However, the accessible clinically applied heating system suffers from some limitations including unsophisticated temperature monitoring devices, no real-time temperature sensing, limited access to the imaging guidance system [35, 38], or difficulty in controlling the heat distribution within specific volume [39]. These limitations restrict its global use in oncology.

Two main themes are prevalent in developing and improving the heating system in hyperthermia localized treatment; (1) the development of real-time temperature monitoring system; and (2) the achievement of localized and control therapy.

\section{1. 1 Real-time Temperature Monitoring Systems:}

Efficient sensing systems in hyperthermia localized treatment are very important to help deal with cancer, the second most common cause of human deaths worldwide according to the annual report of the American Cancer Society in 2018 [40]. Non-invasive thermometry techniques are more desirable than invasive techniques for in vivo sensing. Because the non-invasive thermometry techniques are capable of overcoming some of the challenges associated with the design of the invasive-sensing methods including nontoxicity, biocompatibility, stability over long periods of time, and multipoint measurements [35, 36, 41- 43]. 
Currently, no commercially approved non-invasive sensing system is available due to a number of issues including technical complications, the complex needs of phantoms for calibration, as well as expensive equipment [37]. On the other hand, many of the invasive techniques, FOS-based sensors including FBGs devices, have been approved by Food and Drug Administration (FDA) to be utilized clinically; as well, FBGs sensors are commercially available. Therefore, FBGs sensors are one of the most promising sensing techniques for potential application in the near future. FBGs sensors also satisfy the four properties commonly utilized to evaluate biomedical sensors [29, 44]. These properties are high sensitivity, stability, safety, and selectivity of controllable measurements depending on the applied sensing system.

\section{1.2 Achievement of Localized and Control therapy, researchers' goal:}

The significance of localized therapy approaches resides in the protection of normal tissues, the prevention of further complications which may result in additional required health care, and the maximized efficiency of localized treatment. New cancer heatactivated drugs are being developed to only become active in the target zone undergoing hyperthermia $[1,48,49]$. Carbon nanotubes could potentially be used as a drug delivery vehicle due to its unique electrical properties and large surface-to-volume ratio [52]. Further localized treatment techniques based on heating metal nanoparticles or carbon nanotubes using intense lasers have been widely investigated [53-63]. These metal nanoparticles are usually injected into the target zone to work as energy absorbers and cause localized lethal damage. A recent study has shown a high degree of thermal therapy localization which can be achieved by using gold/gold sulphide (GGS) nanoshells and NIR pulsed-laser [53]. Their numerical simulation enables the ability to estimate the heat 
distribution in a tumor. The validity of the numerical simulation is then investigated experimentally in a phantom made of agarose and intralipid (Figure 1.3). Nevertheless, serious drawbacks must be resolved before enabling their clinical implementation. These drawbacks include non-biocompatibility and possibility of drug leakage before reaching the target.
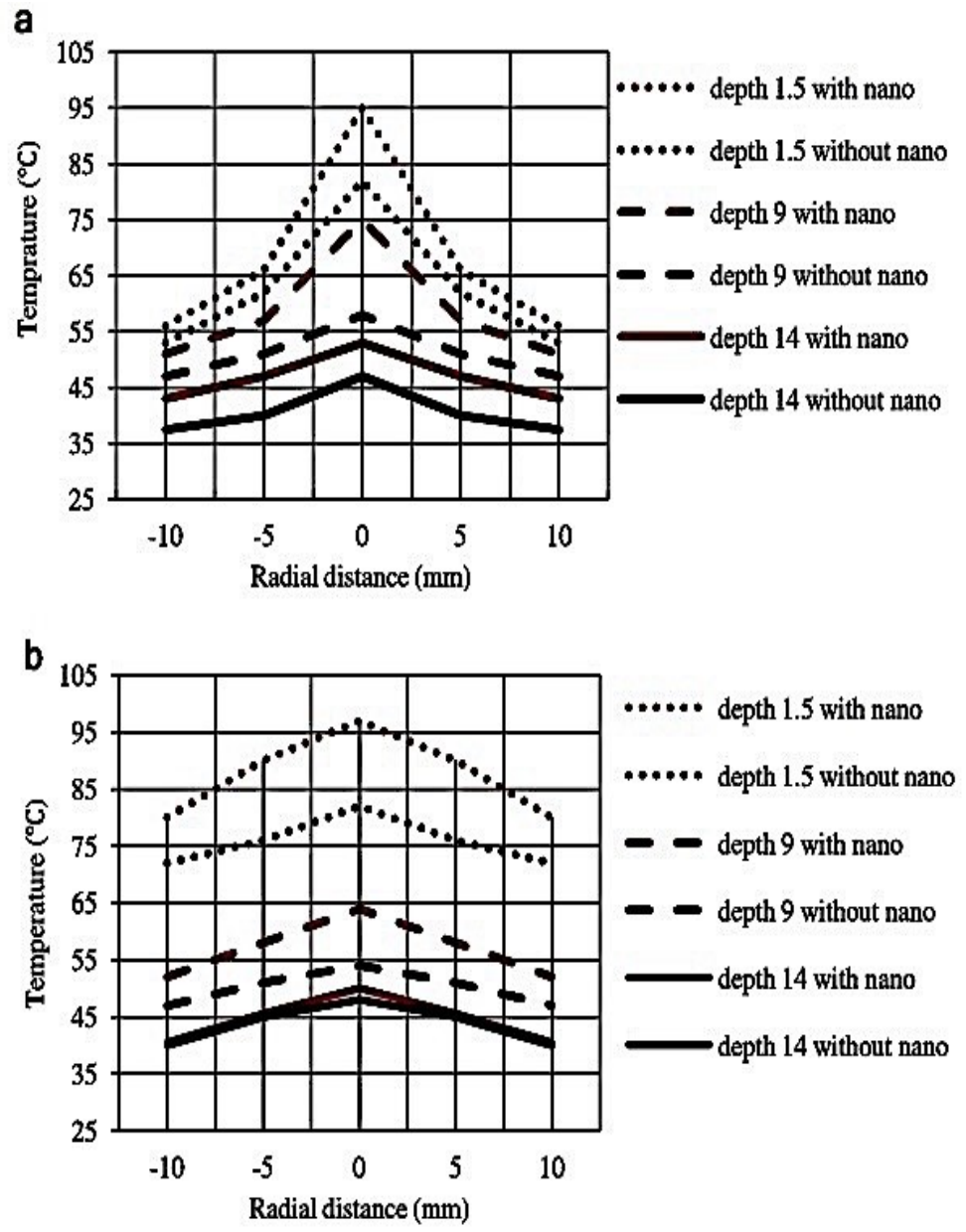

Figure 1.3. An example of one of the treatment' goal in oncology, using nanoparticles to achieve more targeted treatments (by absorbing more energy and hence causing a larger temperature increase ) and less harmful overall to the body. Temperature increase at different depths with and without the nanoparticle (a) simulated results, and (b) experimental results [53].

On another aspect of cancer therapeutics by using non-ionizing radiation, $\mathrm{THz}$ radiation has also been considered in cancer therapy to kill or limit cancer growth and in early 
detection of cancer cells [64]. Molecules including proteins and DNA have distinct signatures to $\mathrm{THz}$ radiation because of their unique molecular vibrations and rotational energy levels between weakly-bound molecular entities with hydrogen bonds and weak interaction (van der Waals forces, for example); these signatures allow $\mathrm{THz}$ rays to directly detect any deficiency in their structure caused by tumor. Under certain conditions including long exposure duration, high power, and specific frequency of $\mathrm{THz}$ radiation has the ability to cause damage in the malignant growth. Researchers are continuously trying to improve the performance of various hyperthermia treatment techniques. Case in point, after almost 59 years of development in magnetic induction hyperthermia technology, it has finally entered a clinical phase and animal-based test stage.

\subsection{Summary of the Thesis:}

FBGs outdo to some extent the performance of many other devices and prove their position in the market because of their characteristics and design feasibility. In addition, FBGs are a powerful device with great potential in various applications. Accordingly, we aim to develop a smart active heater based on one of the configurations of these diffractive gratings: namely tilted-FBGs (TFBGs). This device promotes coupling to certain modes, such as radiation modes, in addition to sharing FBGs' optical properties and advantages. The structural shape and optical properties of the TFBG allow it to be used for two purposes:

(1) as a heat source to induce localized temperature increase.

(2) as a thermometer to control the resulting increase in temperature during hyperthermia. The tilt angle of the grating allows coupling to cladding modes and absorption of light by the amorphous carbon pigment coating material generates heat. Monitoring the Bragg 
resonance shifts of the grating is then enabled the determination of the temperature increase within the treated volume.

We show the efficient performance of this active heater at both cellular and tissue levels. The in vitro hyperthermia-induced cell-killing studies demonstrate that MCF-10A cells are more sensitive to heat than HEK293 and MCF7 cells. Also, we observe that the dead MCF-10A cells are surrounded by a necrotic liquid while the dead HEK293 and MCF7 cells are not indicating different responses to heat resulted in two types of cellular death.

We then show the ability of this device to cause control hyperthermia in tissue-like material and ex vivo studies. Further, we build a computational model to predict the temperature increase and extent of damage in living tissues receiving hyperthermia treatment by the TFBG-based active heater. We obtain a good degree of agreement between the experiment and theory results with a lesion size of $5 \times 12 \mathrm{~mm}$ and $4.87 \times 11.6$ $\mathrm{mm}$ in the phantom tissue and $7 \times 15 \mathrm{~mm}$ and $8.8 \times 14.3 \mathrm{~mm}$ in the ex vivo studies at pumping power of $1.8 \mathrm{~W}$ for 10 minutes respectively.

After obtaining good agreement between the experiment and theory results, we simulate the performance of the TFBG-based active heater to induce localized treatments under the presence of blood perfusion. The blood acts as a heat sink at the gratings' surface by reducing the temperature increase value by $11 \%$ when compared to the same heating parameter without considering the effect of blood perfusion. On the contrary, the effect of blood was insignificant on the temperature increase resulting from the flow of heat from the active heater surface into the heated target and hence on the damaged volume. 
Finally, we also investigate the TFBG-based active heater thermal stability and the heating ability when it has been packaged in a needle filled up with a conductive material. Hyperthermia-induced lesions by the packaged active heater were in order of $7.9 \times 14.5 \mathrm{~mm}$ and $7.583 \times 5.24 \mathrm{~mm}$.

Two laser sources are used to fabricate the gratings utilized in this thesis work. We use the femtosecond laser to write the FBGs used to evaluate the thermal stability of the diffractive gratings. The diffractive gratings used to induce hyperthermia are written by the excimer laser, because the grating written by femtosecond laser tends to be more stable than the devices written by the excimer laser. In the heat-induced hyperthermia studies we did not need high thermal stability devices. However, the thermal stability of the TFBG-based active heater is a very important factor for future practical applications.

For simplicity we evaluated femtosecond FBGs because most of the diffractive gratings written under the same conditions have similar thermal stability. TFBGs written by the femtosecond laser have shown high thermal stability up to $800{ }^{\circ} \mathrm{C}$ [65], which can be increased to $1500{ }^{\circ} \mathrm{C}$ if the tilted gratings are written in sapphire fibers [66]. In addition, the regeneration process has been observed in seed gratings written with various tilt angles including 4 and 8 degrees [67]. In this thesis work, we studied the short- and longterm thermal stability of the third-order gratings because these devices have shown high thermal stability along with a large index change which cannot yet be obtained from the regenerated gratings [70]. The third-order gratings were stable over a short period of annealing at $1000^{\circ} \mathrm{C}$. In contrast, the regenerated gratings were thermally stable over a one-month period of annealing. 


\subsection{Organization of the Thesis:}

A short introduction of hyperthermia therapy techniques, application of thermometer devices, and types of phantoms are described in Chapter 2. The fabrication technique and the work principle of TFBGs are described in Chapter 3. Chapter 4 explains the material and methods used to accomplish this thesis work followed by the experimental results in Chapter 5. The computational model descriptions with a brief introduction of COMSOL are expounded in Chapter 6. I compare the experimental and theoretical results in Chapter 7 and demonstrate the heating efficiency of the TFBG-based active heater under the effect of blood perfusion in human liver. Finally, Chapter 8 concludes this thesis work with some thoughts about the possible future works. 


\section{Chapter 2: Local Hyperthermia Therapy}

With an aim to establish certain conceptual aspects of local hyperthermia therapy, this chapter provides an overview of the state-of-art local hyperthermia cancer treatment systems including heat sources, applied thermometry devices in the thermal therapy field, as well as examples of artificial environment "phantoms" used to evaluate such treatment systems. Stand on the topic of this thesis; the discussion in this section is limited to the invasive thermometry techniques utilized in hyperthermia cancer treatment. Further, since the groundwork of this thesis is based on fiber optic gratings, special focus is given to the role of FBGs' temperature-based sensors and their development in local hyperthermia therapy.

\section{1 Introduction to Local Hyperthermia (LHT):}

Local hyperthermia therapy has been applied to cause a lethal localized volume in a target range between less than 3 to $5 \mathrm{~cm}$ and up to 5 to $6 \mathrm{~cm}$ along the axial side of the applicator $[1,4,5,15-17,25,30,34,39,71,72]$. The location of the treatable cancerous growth by this method is usually superficially, interstitially including head, neck, and prostate; or else within a cavity in the body such as the oesophagus or the rectum. Local hyperthermia can be performed by several means such as microwave, radio frequency, and ultrasonic waves. To ensure deep and homogenous temperature disruption in the treated zone, a cooling system using water for example is often applied to the applicator $[71,73]$. Each one of these means used to generate heat has contributed to hyperthermia therapy in treating specific types of cancerous growth with limitations. However, 
hyperthermia therapy based on radiofrequency is used clinically and is more developed than the other energy sources implemented in local hyperthermia.

In accordance to the temperature regime, local hyperthermia has been classified into the following three types: thermotherapy, high-temperature therapy, and thermal resection therapy $[30,72]$. Thermotherapy is a very prolonged procedure used to cause irreversible damage in tumor tissues via increasing the temperature from $42{ }^{\circ} \mathrm{C}$ to $46{ }^{\circ} \mathrm{C}$. The high temperature hyperthermia therapy rationale, also known as thermoablation, is based on causing necrosis and coagulation of tumor tissues. The range of temperatures applied in

the thermoablation therapy ranges from $46{ }^{0} \mathrm{C}$ to $70{ }^{\circ} \mathrm{C}$. A temperature increase of over 70 ${ }^{0} \mathrm{C}$ is utilized in thermal resection treatment to carbonize the malignant tumor. In the method of inducing heat in a specific zone, local hyperthermia therapy has been separated into two predominate regions: local hyperthermia based on direct heating and indirect heating.

\section{1. 1 Local Hyperthermia Based on Indirect Heating:}

Heating in the target volume obtained from hyperthermia therapy from microwave, radio frequency, and ultrasonic waves is indirect [5]. In other words, heat is induced in the tumor tissues through the deposition of the coherent energy from such sources. Most of the applied modern hyperthermia therapy techniques based on energy transformation introduce heat in a target zone.

\section{1. 1. a Microwave heating:}

Frequencies of $2450 \mathrm{MHz}, 915 \mathrm{MHz}$, and $434 \mathrm{MHz}$ comprise the range involved in 
microwave hyperthermia $[1,4,5,20,25,74]$. Applying microwave hyperthermia in the treatment of water-rich tissues, such as liver tissues, has been proven as an effective therapy because microwave frequency has a selective effect on water and watercontaining substance. Generally, the method of performing local hyperthermia therapy through a microwave source has been divided into two branches: external microwave heating and percutaneous microwave coagulation therapy. The division is based on the therapeutic temperature range and the method of applying the treatment.

Cancer cells undergoing convectional microwave hyperthermia treatment are illuminated by microwave which travel through the skin. Due to the high-absorption of the water molecules to the microwaves, the microwaves suffer some degree of attenuation in the propagation process through the skin and the tissues all the way to the target areas. Thus, this technique is restricted to the treatment of superficial tumors and cavity tumors. Notably, this technique is more effective in cavity tumor therapy because of its ability to implement some microwave generators. The excitation of the polar molecules occurring upon the absorption of the microwave radiation in the target area causes high-speed oscillation, and hence heat is generated.

Compared to the above technique, percutaneous microwave coagulation treatment is a more invasive "use of microwave needle radiator" and it relies on a temperature range more than $50{ }^{0} \mathrm{C}$, yet it is more efficient $[30,74]$. This approach entails the utilization of a guiding technique, such as ultrasound or computed tomography scan, to lead the applicator to the target volume. As a result of the applied high temperature, dose deposition can be achieved in a short time, and hence there is less discomfort to the patients. 
The percutaneous microwave coagulation therapy offers several attractive advantages. Owing to the application of high temperature, it results in direct death to the cancer cells through coagulation and denaturation with a spherical coagulation necrosis volume and clear boundary. For instance, a large lesion in order on $20 \mathrm{~mm}$ has been reported through using a microwave antenna operated at $2450 \mathrm{MHz}$ in porcine liver tissues [74]. This localized necrosis also ensures the relative safety of the neighbouring normal tissues. In addition, multiple applicators can be implemented, depending on the applicator size, to enlarge the necrosis zone and shorten the treatment time.

However, this technique is not yet ready for clinical applications for various reasons. The respiration process significantly restrains the efficiency of a treatment session through introducing artifact and error, for example in puncture needle and puncture operation. The influence of the respiration process also changes and shifts the arrangement of multiple applicators needed for the treatment of large tumors. This may result in incomplete treatments of small foci area and hence hinder and complicate the efficiency of the treatment. In addition, because of the physical properties of microwave radiation, a large number of antennas are required in specific volume with an adjacent spacing of 1 to $1.5 \mathrm{~cm}$ to cause the required therapeutic temperature in the whole treated volume. This is highly invasive and cannot be applied clinically. Further multiple drawbacks must be resolved before its use in practical application including the provision of protection needed for personal safety and establishing a usable data base containing the thermal doses required for particular malignant growths.

\section{1. 1. b Radio-frequency hyperthermia:}

A number of frequencies forming the radiofrequency band are being applied in 
radiofrequency hyperthermia oncology including $100 \mathrm{MHz}, 40 \mathrm{MHz}, 27.12 \mathrm{MHz}, 13.56$ $\mathrm{MHz}$, and $8 \mathrm{MHz}[1,4,33,73]$. Radiofrequency therapy works by inducing vibration of ions, charged molecules, and electric doublets in the target zone. Radiofrequency hyperthermia is the method used clinically for the therapy of some types of cancers. This therapy technique has been grouped into two main categories of external radiofrequency hyperthermia and the radiofrequency ablation (RFA). As is the case with microwave hyperthermia, this division has also been made in regard to the method of applying the treatment and the prescription range of the therapeutic temperature.

The external radiofrequency hyperthermia technique works through the production and dissipation of high-frequency oscillation by both joule heat and dielectric loss factors into the tissues "absorption of the electric energy". This method has certain advantages over the conventional microwave hyperthermia method on account of its ability to induce deeper effect; hence, it has been named RF diathermy. Application of radiofrequency hyperthermia is accomplished through two approaches: capacitive heating and inductive heating. The capacitive heating efficiency is much greater than that obtained from the inductive hyperthermia, and now it is the one being applied clinically.

The capacitive RF hyperthermia acts through generating joule heat by focusing the radiofrequency currents between a number of implemented pairs of electrodes or polar plates into the malignant growth. This joule heat is then absorbed into the tissue, thereby causing the cells' death. The disadvantage of this technique is the need to use nonmetallic thermometry. 
The radiofrequency ablation hyperthermia consists of using iconography to guide the applicator to the target zone and an utmost treatment temperature of $120{ }^{\circ} \mathrm{C}$ (Figure 2.1 ). The cluster electrode at the head of the applicator radiates radiofrequency waves. The intermediate-RF waves induce plasma concussion and pyrexia of the tumor tissues, thereby causing an increase in temperatures. The temperature distribution is very high in the treated zone with a temperature of $120{ }^{\circ} \mathrm{C}$ at the centre of the applicator and a lower temperature of $60{ }^{0} \mathrm{C}$ at the boundaries. In addition to the direct killing of cells, this technique has many further advantages such as restricting the growth and dispersion of cancerous growths, high heat-efficiency, and most importantly it is less harmful with less or even no severe or serious side effects compared to other traditional cancer therapy techniques.

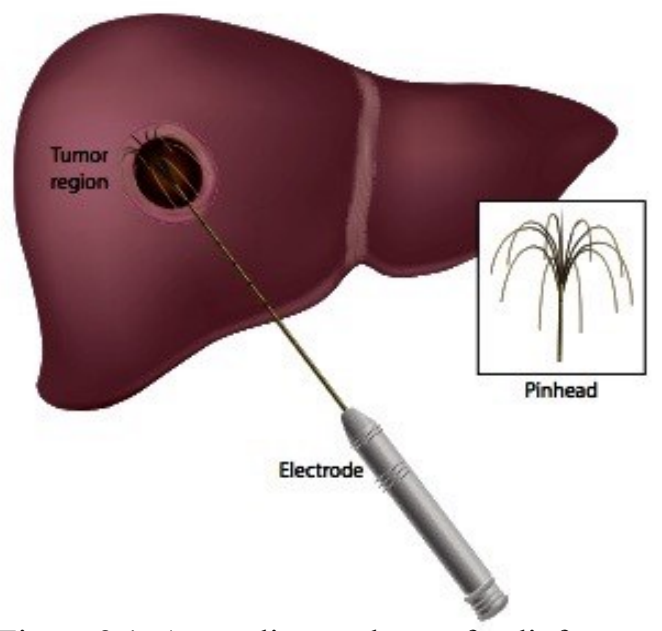

Figure 2.1. An applicator shape of radiofrequency ablation hyperthermia [1].

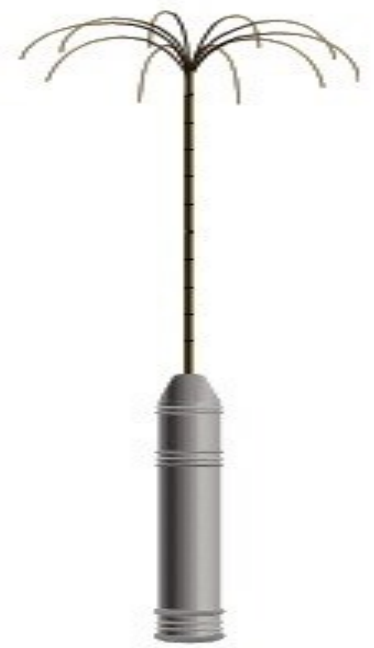

However, the controlling and monitoring devices are not very satisfactory owing to the limitation in measuring the real-time temperature distribution in the treated zone. This affects the efficiency of the procedure. Further, the lesion's maximum diameter which can 
be treated by the RFA technique is less than $5 \mathrm{~cm}$, providing that the location is suitable for implantation such as in the head, neck, or prostate.

\section{1. 2 Local Hyperthermia Based on Direct Heating:}

Direct heating is achieved through direct contact of the human tissue to the heat source “conduction" $[1,5]$. This can be achieved through localized transcutaneous on the skin surface, interstitial tube with turbulent hot water flow or electrically heated probes, heating the whole body in a hot bath, using iron needles, and so on. This process is known as conventional or traditional hyperthermia. This technique is restricted to the depth of treatment (up to $10 \mathrm{~mm}$ with a margin of approximately $20 \mathrm{~mm}$ ) and low therapeutic temperatures.

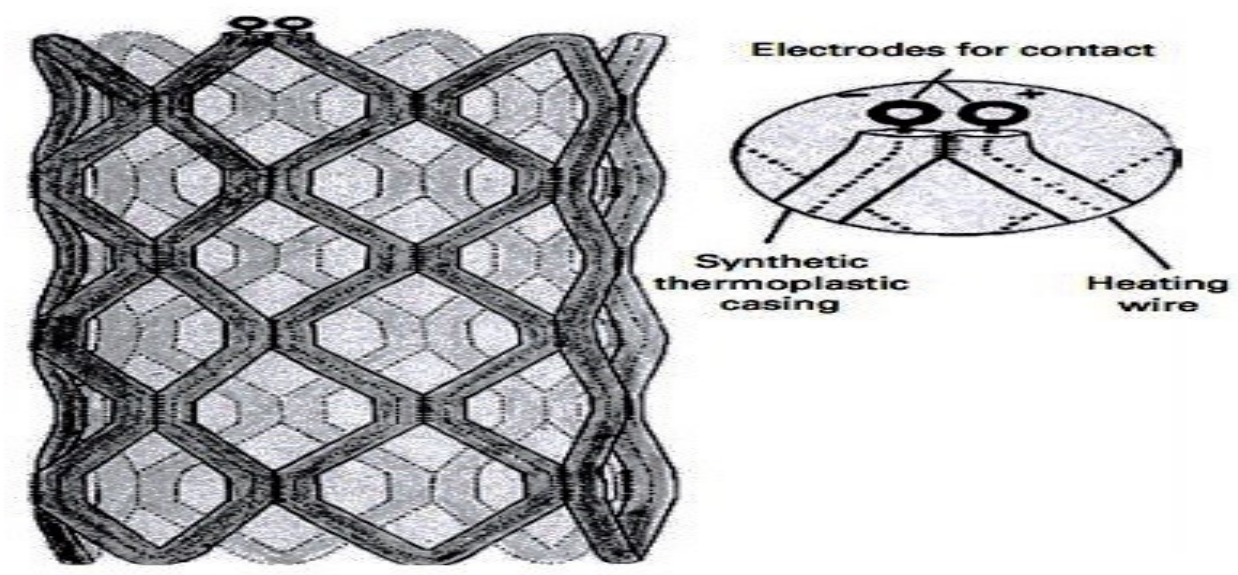

Figure 2.2. An example of applicator used to perform local hyperthermia based on direct heating made of thermoplastic stent wrapped up with a platinum wire [75].

However, in 2006 a group from Germany performed hyperthermia based on applying direct heat [75]. Freudenberg et al. conducted a novel study to examine the therapeutic effect on esophageal cancer of applying hyperthermia through a heatable stent. The researchers assembled the heating device from a thermoplastic stent wrapped up with a platinum wire (Figure 2.2). The heating was induced by applying a voltage in order of $9 \mathrm{~V}$ 
on both ends. The utmost applied temperature without affecting the healthy tissues that was sufficient enough to cause cell death was $46.5{ }^{\circ} \mathrm{C}$, which led to an increase in the tissue's temperature to $42.5{ }^{\circ} \mathrm{C}$ and $41{ }^{\circ} \mathrm{C}$ at a depth of $12 \mathrm{~mm}$ and $16 \mathrm{~mm}$ respectively (Table 2.1).

\begin{tabular}{lcl}
\hline $\begin{array}{l}\text { Temperature } \\
\text { of the stent }\end{array}$ & $\begin{array}{l}\text { Penetration } \\
\text { at } 42.5^{\circ} \mathrm{C}\end{array}$ & $\begin{array}{l}\text { Penetration } \\
\text { at } 41^{\circ} \mathrm{C}\end{array}$ \\
\hline $44^{\circ} \mathrm{C}$ & $7 \mathrm{~mm}$ & $10 \mathrm{~mm}$ \\
$46.5^{\circ} \mathrm{C}$ & $12 \mathrm{~mm}$ & $16 \mathrm{~mm}$ \\
$48^{\circ} \mathrm{C}$ & $14 \mathrm{~mm}$ & $17 \mathrm{~mm}$ \\
$50^{\circ} \mathrm{C}$ & $17 \mathrm{~mm}$ & $20 \mathrm{~mm}$ \\
$52^{\circ} \mathrm{C}$ & $20 \mathrm{~mm}$ & $23 \mathrm{~mm}$ \\
\hline
\end{tabular}

Table 2.1. Temperatures as a function of the penetration depth [75].

\subsection{Thermometry:}

The development of temperature monitoring tools for hyperthermia originated in the 1970s [1, 3- 5, 26-29, 44, 76]. Accurate monitoring of the temperature distribution in a target allows for control of the volume of coagulation without damaging the healthy neighbouring cells. Thermocouple is one of the oldest applied techniques of temperature measurements in hyperthermia cancer treatment. This technique is still widely used for medical temperature measurements owing to its low cost, reliability, and rapid response $[27,31,72,77,78]$. However, because thermocouple consists of metallic parts such as a receiver antenna, this technique is not adequate for use in a number of applications including microwave ablation, radiofrequency ablation, or laser hyperthermia therapy. These applications require invulnerability from electromagnetic interference of current and voltages and energy absorption to avoid error in the temperature measurements. Energy absorption by the metallic parts causes a significant increase in the sensor 
temperature, thus resulting in a much higher reading temperature than the actual heating temperature.

Two approaches have been considered to resolve this issue $[3-5,19,26,27,76,79,80]$ : 1) a thermocouple with some modifications to overcome error in the measurements due to self-heat or electrical interference; and 2) fiber-optics based sensors, either glass or plastic.

\section{Type Metal (Positive-Negeative)}

\section{Setetedks Coefficient (uV/C) NIST Range ('C)}

\begin{tabular}{|c|c|c|}
\hline \multicolumn{2}{|c|}{ Platinum (30\% Rh)-Platinum (66\% Rh) 5.96 a a $6000^{\circ} \mathrm{C}$} & $0+1820$ \\
\hline Cromel-Costantatana & 58.67 to $10^{\circ} \mathrm{C}$ & $.270+1000$ \\
\hline Irön-Costantana & 50.38 a $10^{\circ} \mathrm{C}$ & $-210+1200$ \\
\hline Cromel-Alumel & 39.45 at $0^{\circ} \mathrm{C}$ & $.270+1372$ \\
\hline Copper-Coslantana & 38.75 औ $10^{\circ} \mathrm{C}$ & $-270+400$ \\
\hline
\end{tabular}

Table 2.2. The available thermocouples in the market according to the ANSI classification [5].

The thermocouple is a non-passive transducer which relies on the thermoelectric effect of their circuit to obtain a temperature-dependent voltage produced between the two wires' junction. Table 2.2 indicates some types of thermocouples that are available in the market according to the ANSI classification $[5,72]$. The T-type thermocouple is utilized in hyperthermia cancer treatment. With this thermometry technique, an amplifier must be used in the temperature measuring system in order to amplify the output voltage. This inevitably increases the output voltage from $0.23 \mathrm{mV}$ to $2 \mathrm{mV}$ and hence obtains an accuracy of $0.1{ }^{0} \mathrm{C}$ in the measured temperature. The usual sensible temperature range is 
between -200 to $1500{ }^{\circ} \mathrm{C}$ (Table 2.3). Further, no real time temperature measurements can be obtained, meaning temperature measurements must be accomplished when the heating source power is in the off mode. In addition, some modifications must be made on such an active thermocouple to insulate the actual measured temperature from uncertainty or noise from certain factors which may be present in the sensing environment. For example, a thin layer of epoxy is applied on a T-type thermocouple to provide electrical insulation while studying the effect of a near-infrared laser beam when focused on a rat brain [81].

\begin{tabular}{clcl}
\hline Type & Thermocouple & Sensitivity $\mu \mathrm{V} /{ }^{\circ} \mathrm{C}$ at $37^{\circ} \mathrm{C}$ & Useful range ${ }^{\circ} \mathrm{C}$ \\
\hline Type T & Copper/Constantan & 42 & -150 to +350 \\
Type J & Iron/Constantan & 53 & -150 to +1000 \\
Type K & Chromel/Alumel & 41 & -200 to +1200 \\
Type E & Chromel/Constantan & 62 & -200 to +900 \\
Type S & Platinum/Platinum-rhodium & 6 & 0 to +1500 \\
\hline
\end{tabular}

Table 2.3. Diffrecnt type of thermocouples with their sensing characteristics [72].

Fluorescence-based temperature sensor is one of the earliest fiber optics sensors which was patented by Luxtron Corporation of Mountain View in the early 1980s [80]. Now, LumaSense Technologies have commercialized fluoroptic sensors for medical applications including temperature monitoring during MRI hyperthermia [27, 82, 83]. Its sensing principle is based on analyzing the change in temperature by monitoring the decaying time of the luminescence which becomes excited by radiating rare-earth fluorescent elements through an LED radiation (e.g. Figure 2.3) [84]. The fluoroptic sensors are able to perform temperature measurements from $-25{ }^{\circ} \mathrm{C}$ to $300{ }^{\circ} \mathrm{C}$ with 
accuracy of $0.2{ }^{0} \mathrm{C}$, resolution of $0.1{ }^{\circ} \mathrm{C}$, and response time of approximately 1 second [77, 82]. Although the measurements suffer from some artifacts, fluoroptic sensors are still in use. However, their applications are limited by the artifact on the temperature measurements caused by the self-heating of black-pigments which are used to coat the fluoroptic sensor [27].

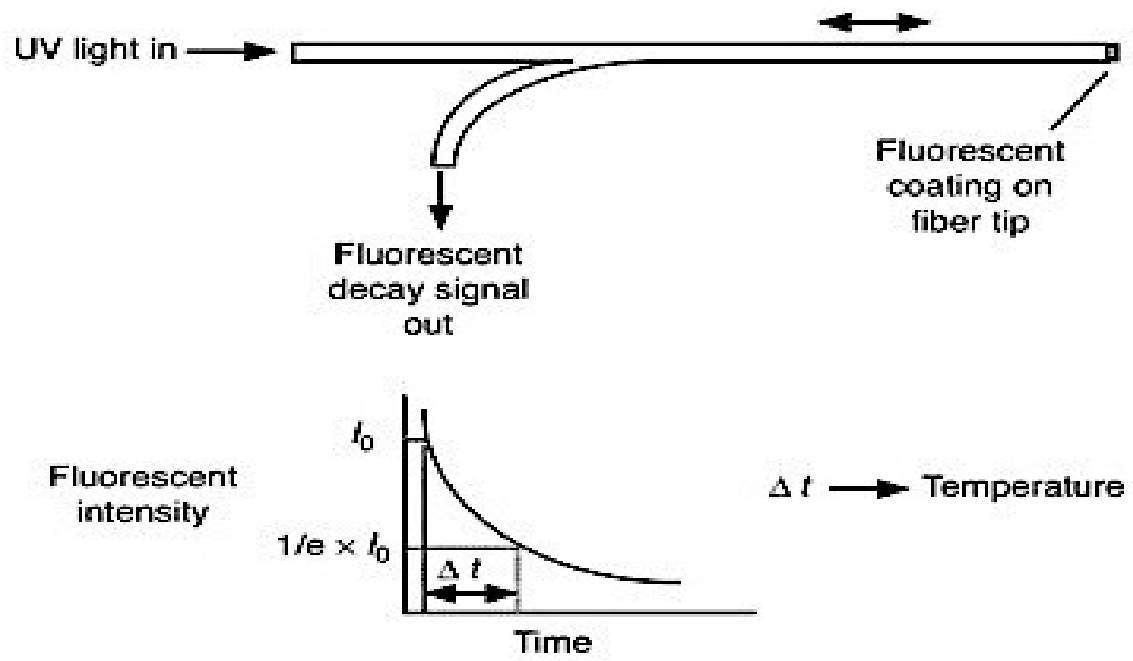

Figure 2.3. Schematic diagram of fluorescent structure, and temperature as a function of the decaying time of the fluorescent [29].

In addition to the complications associated with these sensors, a serious problem has been raised consisting of the operating nature of these common sensors including thermocouples and fluoroptic sensors. They are point sensors delivering measurements from only one point in the treated area, meaning temperature readings over a small volume in the tumor rather than temperature disruption within the tumor $[1,3-5,19,37]$. This feedback is neither reliable nor informative, because it depends on the sensor size and position on the implemented area. Temperature measurements from multipoint "special temperature readings" are indispensable for sufficient treatment and protection of healthy tissue. 
Correspondingly, thick bundles of single point temperature readings have been designed to fulfill this need. For example, fluoroptic sensors are being used as a multipoint-sensor system through multiple-channels pumped by the same source [27]. However, this development requires increasing the probe size, which has resulted in discomfort and serious risks to the patient in addition to severe restrictions applied by the clinical health requirements and the high cost.

FBG-based temperature sensor is an ideal sensor for this task due to its biocompatibility, capability of multiplexing sensing, immunity to electromagnetic interference, small size, and so on $[85,86]$. But most importantly, FGB-based temperature sensor has the ability to provide a real time feedback in order of number of milliseconds after packaging. The response of a specific volume undergoing hyperthermia is not as predictable as in the case of theoretical simulation because of various factors. For example, the perfusion rate is a function of heat, and so blood flow varies at different points during the heating. Although various theoretical methods have been proposed and used to estimate the temperature distribution around the applicator, they are not very reliable in clinical applications, and hence the need for real-time monitoring arises [87].

\section{2. 1 FBGs in Hyperthermia:}

In 1997, Rao et al. were the first to recognize the potential of FBG sensors in such applications. They developed a novel FBG sensor array of 'a strain-free probe' to assess the sensors' performance and monitor temperature in-situ under the application of high magnetic field [88]. The FBG sensors are enclosed in a protective sleeve of a diameter of 
$0.5 \mathrm{~mm}$ for direct temperature measurements with a resolution of $0.1{ }^{0} \mathrm{C}$ and an accuracy of $0.2{ }^{\circ} \mathrm{C}$ over a temperature range between $30{ }^{\circ} \mathrm{C}$ to $60{ }^{\circ} \mathrm{C}$. The thick diameter of the sleeve is chosen to tolerate the transverse stress without transferring it to the probe. Twelve years later, Intelligent Optical System, Inc developed and commercialized the optical fiber temperature (OFT) multiple point sensors [27]. The OFT sensors can elevate temperature monitoring and profiling in a treated area of approximately $0.5 \mathrm{~cm}$ by providing feedback from 10 points with high-resolution without requiring thick bundles of point sensors. This can reduce the treatment complications one of which damaging the healthy tissue surrounding the tumor. Also, owing to its small size ( $0.25 \mathrm{~mm}$ thick), OFT sensors can be utilized in a broad range of clinical conditions.

The only in vivo trial took place 16 years ago at the Cancer Research Institute in Perth, Australia [89] where temperature profiling took place from multipoints in the kidneys and livers of rabbits undergoing hyperthermia treatment by inductive heating of metallic implants. The probe was designed by placing the multi-grating FBGs sensors in a $1 \mathrm{~mm}$ outer diameter surgical catheter (Figure 2.4).

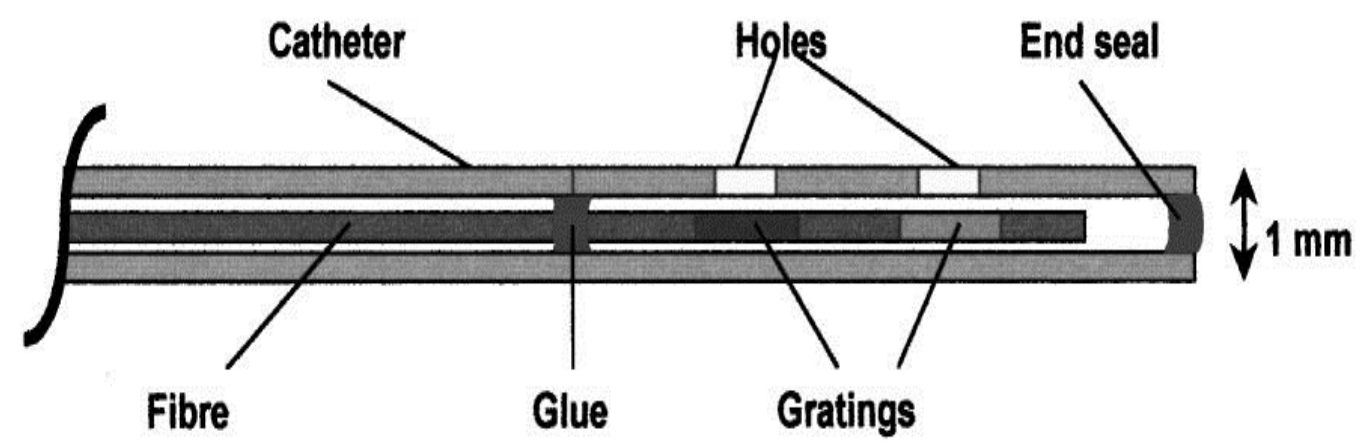

Figure 2.4. Schematic diagram of the applicator used in vivo to measure the temperature increase the kidneys and livers of rabbits during hyperthermia [89]

Above each grating a hole in order of $1 \mathrm{~mm}$ was cut through a portion of the surgical catheter wall in order to increase the sensitivity and the recompense time of the 
temperature measurements. Then the probe was inserted within a hypodermic needle just to enable the insertion of the probe into the rabbits' kidneys and livers. The treatment and hence the temperature monitoring was started after withdrawing the needle and leaving the probe in the desired place. To ensure the device performance, the output measurements of the sensors array were compared with those of the Fluor optic probe (Figure 2.5). This study highlighted crucial points to consider in future FBGs sensing system design, including: (1) place the instrument of FBGs monitoring signal away from the treating site to avoid interference, in this case, between the spectrometer and the large current inside the inductive heating system; and (2) more in vivo study as well as the need for developing theoretical models to estimate the effect of the background tissue temperature on the real increase in the induced temperature.

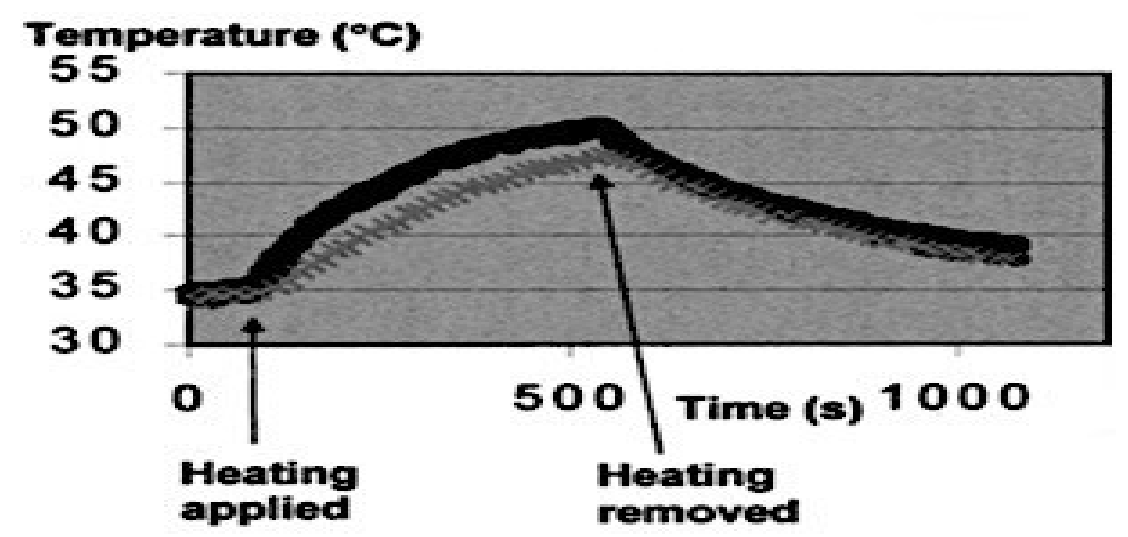

Figure 2.5. Compared FBGs (circles) output measurements with those of the Fluor optic probe (crosses) [89].

\section{2. 1 a FBGs problems:}

One problem that impacts the use of FBGs as temperature sensors is the high cost of the equipment required for the signal detection [3, 4, 26-29, 78]. Recently, an additional important issue has been addressed by D. Polito et al. related to packaging of FBGs sensors [45]. Currently the first issue, the high cost of the equipment required for the 
signal detection to prevent thermal crosstalk, is not a significant issue in the medical field because hyperthermia is an essential therapeutic technique in oncology; in addition to the urgent need to such temperature sensor regardless to its invasive nature. However, it is noteworthy to mention some of the efforts engaged to economize the sensing system cost.

The high cost of instruments imposes from the need to use multiplexed sensors, because spectrometer with resolution of sub-picometer is required for wavelength shift detection. The resolution in many of the conventional spectrometers is in the range of 0.01 to 0.1 $\mathrm{nm}$; therefore, multiplexed sensors system suffers from a thermal cross talk between adjacent signals which govern the spatial resolution of the system $[78,90]$. Other approaches have been taken into account to overcome both problems, and hence enable the use of FBGs array in practical applications. These techniques can be classified based on their operating principles including edge filter, tunable filter, inter-ferometric scanning, and dual-cavity interferometric scanning methods [77]. For instance, one study adopted a fiber interferometer method of unbalanced Mach-Zehnder interferometer which have been applied in the sensing system, also, to deal with strain-temperature discrimination $[77,91]$.

To avoid temperature crosstalk between the adjacent signals, a monochromator was added to the Michelson interferometer for demultiplexing the return signals from the FBGs sensors [77, 91]. Including the monochromator in the sensing system enabled the recovery of the wavelength shifts between the adjacent signals. Thus, the spatial separation between two neighboring diffraction spots focused on the output plane of the monochromator is much larger than the size of the spots and the effective detection area of the detectors. Their sensing system is capable of thermal characterization with a 
resolution of $0.1{ }^{\circ} \mathrm{C}$ and an accuracy of $0.2{ }^{\circ} \mathrm{C}$ over a temperature range of 30 to $60{ }^{\circ} \mathrm{C}$. Later, the same researchers applied fiber interferometer Fabry-Perot filter (FPF) for wavelength shift detection and wavelength division multiplexing of the FBG sensors. The tunable FPF is utilized for temperature measurements ranging from $25{ }^{\circ} \mathrm{C}$ to $60{ }^{\circ} \mathrm{C}$ under a magnetic field of $4.7 \mathrm{~T}$ using a Nuclear Magnetic Resonance (NMR) [88, 92]. The simplicity and compactness are the most distinctive merits of this sensing system. The sensing device shows a temperature resolution in order of $0.1{ }^{0} \mathrm{C}$ over time measurements of 1.6 second, but the accuracy is reduced to $0.8{ }^{\circ} \mathrm{C}$. The cause of the reduction in accuracy is interpreted as resulting from the nonlinearity of the piezoelectrical transducer (PZT) in the FPF. An easier and more cost-effective approach was introduced by G. C. Askins et al. [90]. Their approach is based on utilizing a linear CCD array and A/D converter in the sensing system in which the FBG signal is recorded by the A/D converter and the center of each FBG is determined by fitting the obtained spectrum using a standard Gaussian function. The sensing system shows a resolution of $0.1{ }^{\circ} \mathrm{C}$ and an accuracy of $0.2{ }^{\circ} \mathrm{C}$ over a temperature range of $20-50{ }^{\circ} \mathrm{C}$, yet the measurements speed is reduced. Later this system has been tested in vivo on tumors in the livers of rabbits undergoing hyperthermia therapy [89].

The packaging matter has been overlooked in most studies of the development of FBGs sensors for medical applications. In many studies, FBGs have usually been inserted into a catheter by opening some punctures to avoid isolating the gratings from the sensor medium and then inserted into a needle or a catheter filled up with certain conductor materials [48]. However, insufficient information has been provided about the properties of these materials. 
One of the important reasons has been identified as the small size of FBGs sensor, it has been intended to either attach the sensor to the applicator needle, like Radiofrequency needle, or through a separate insertion. In addition to that, some studies have investigated the influence of the strain in the temperature measurements though testing FBG performance in direct contact with an ex vivo tissue [27]. It was concluded that the error resulting from the mechanical strain is negligible on the temperature measurements. However, FBGs sensors cannot be applied for clinical practice without appropriate packaging.

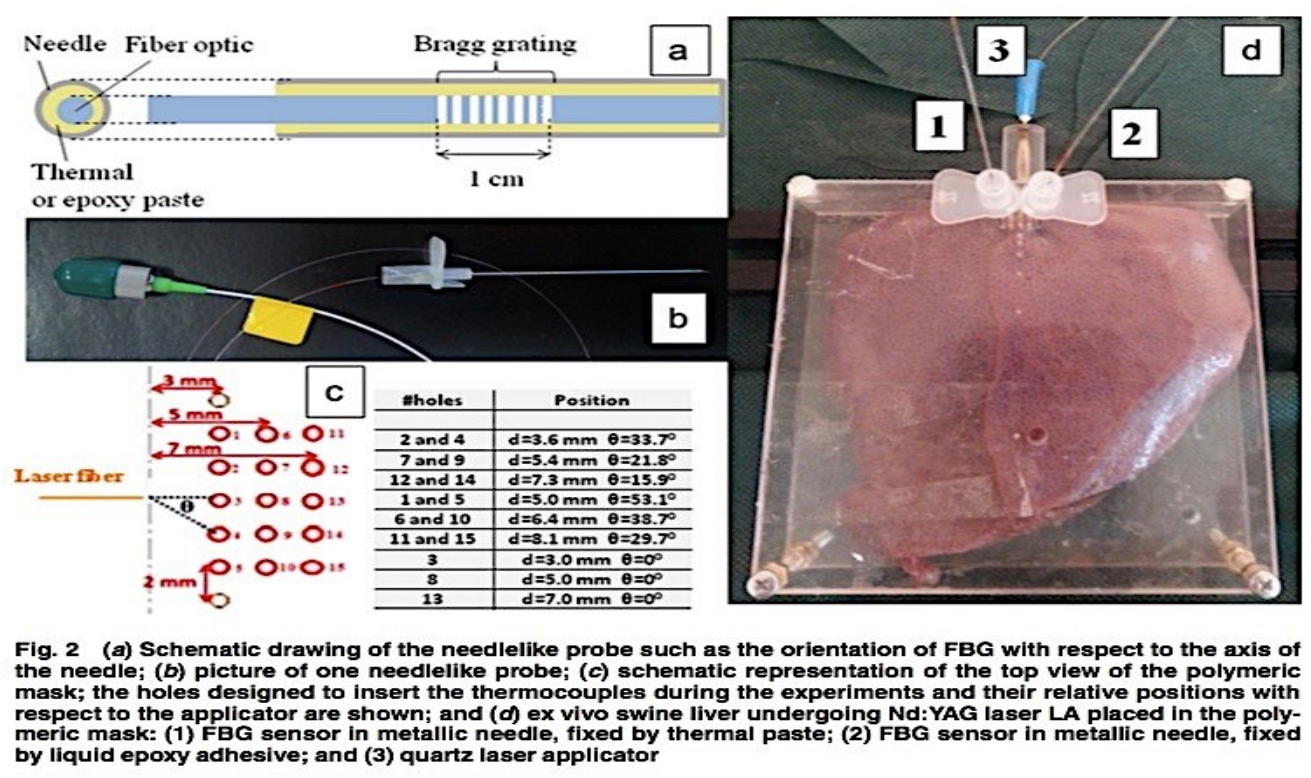

Figure 2.6. Schematic diagram of FBGs thermometry's and their positions in a liver phantom [45].

Polito et al. have investigated the thermal sensitivity and response time of FBGs inserted into a swine liver at different positions undergoing laser ablation hyperthermia (Figure 2.6) [45]. To achieve the optimum thermal sensitivity out of their proposed probe, the needle was filled up by conducted materials: an epoxy liquid and a thermal paste. The measured thermal sensitivities are $0.027 \mathrm{~nm} /{ }^{0} \mathrm{C}$ and $0.01 \mathrm{~nm} /{ }^{0} \mathrm{C}$ respectively for both 
conducted materials. These devices manifest a response time in order of $100 \mathrm{~ms}$ which is lower than that of bare FBGs by $57 \mathrm{~ms}$. This is still very fast compared to the sufficient required response time in the field which is less than 4 seconds [5].

\section{2. 1 b Role of FBG in the Development of Hyperthermia:}

Researchers are continuously trying to improve the performance of various hyperthermia treatment techniques $[48,85]$. Many of these studies have used FBGs sensors owing to their appealing properties which may reduce the system size and cost, provide real time feedback, and facilitate an understanding of the response of a cancer to therapy. To optimize the thermal dosimetry of an applied hyperthermia treatment technique, an assessment of the thermal effects on a target area undergoing hyperthermia - such as estimation of the treated volume size and temperature distribution within and around that target - is necessary. For example, one study used FBG sensors to explore the performance of LITT ablation on pancreas tumor treatment. Because of the high accuracy and sensitivity of FBG sensors, the tissue temperature distribution around the applicator as a function of the laser power was obtained experimentally with a good agreement with the theoretical model (Figure 2.7) [93].

Experimentally validating this theoretical model can allow for an estimation of the optimal laser settings such as the required time and power to treat a specific volume. That is an essential factor in the development of hyperthermia treatments techniques. Recently, double-applicator LITT model has been proposed to increase the treatment efficiency by increasing the lesion size and reducing the treatment time [94]. 12-FBG sensors with 1.5T-MR imaging system have been utilized to assess the performance of this model. Within 

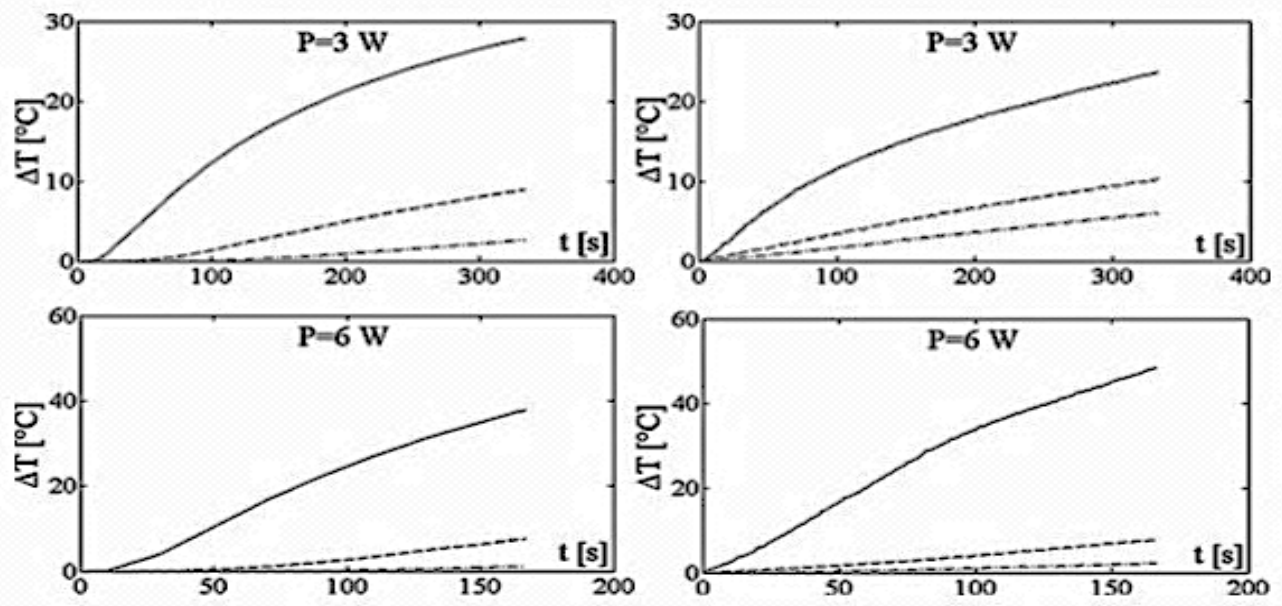

Figure 2.7. Comparison between the theoretical (on the left) and experimental (on the right), temperature measurement at different distance from the applicator, continuous line $(5 \mathrm{~mm})$, dashed line $(10 \mathrm{~mm})$, and dashed-dotted line (15mm) [93].

a volume of $\left(0.91 \pm 0.09\right.$ vs. $\left.0.95 \mathrm{~cm}^{3}\right)$, two temperature distribution profiles around the applicator have been observed: (1) maximum increase in temperature of order of $60{ }^{\circ} \mathrm{C}$ in the area facing the end part of the applicator tips; and (2) increase in temperature of order of $40{ }^{\circ} \mathrm{C}$ and $30{ }^{\circ} \mathrm{C}$ along the other end of the applicators. Further, it has been anticipated that different energy can be deposited at different locations in the tumor as needed by changing the distance between the laser applicators. This could potentially solve the issue with the pullback technique which is the method that has been used clinically up until now to increase the volume of the treated area and to deal with the different shapes of tumors in the expense of treating time. 
Real-time temperature profiling by an array of FBGs sensors has been applied in a study to understand the response of the liver (porcine samples) undergoing Radiofrequency thermal ablation $[20,95]$. The device consists of five gratings written in a single fiber with a length of $0.5 \mathrm{~cm}$ and with $1 \mathrm{~cm}$ spacing between them. This distance between the separation points are in the range of the required distance ' in order of 1 to $1.5 \mathrm{~cm}$ for sufficient treatment. It has been found that the temperature disruption along the applicator is not a function of time in the liver (Figure 2.8). That was attributed to the difference in the electrical properties of the liver (tissue impedance). Thus, the efficiency of Radiofrequency thermal ablation treatment may be improved by monitoring the treatment procedure in real-time and/or designing a tool to estimate the tissue impedance before starting the treatment.

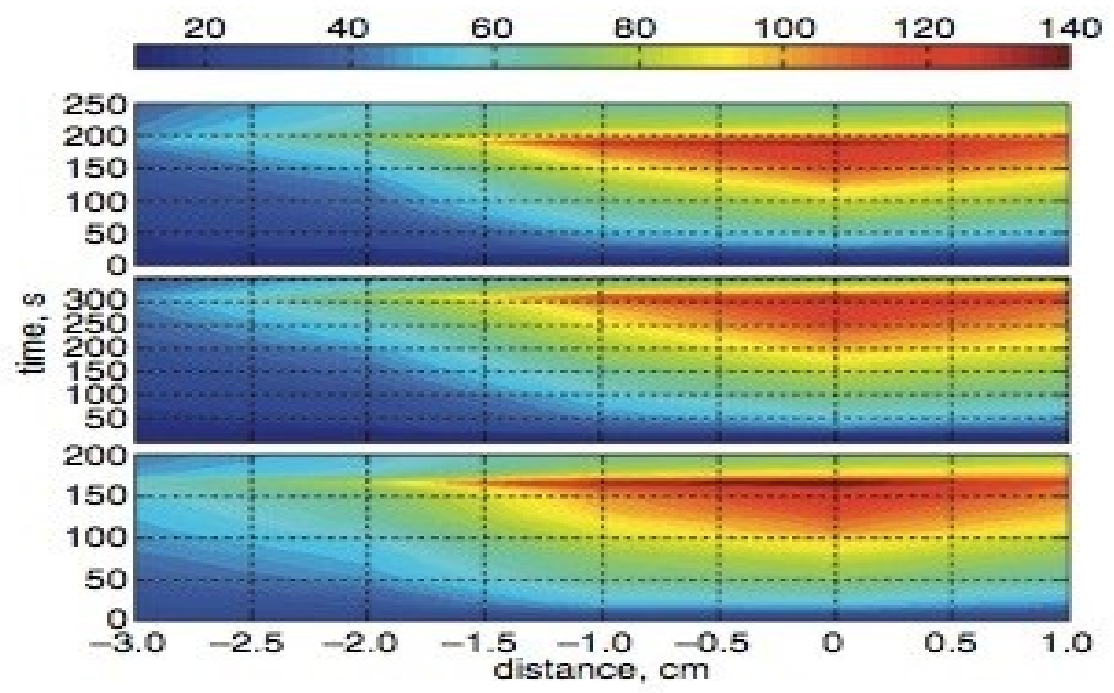

Figure 2.8. Temperature distribution along longitudinal axis as a function of time for three different RFTA experiments with applying the same power in order of $20 \mathrm{~W}$ RF: the temperature disruption along the applicator is not a function of time in the liver due to difference in the liver impedance [95]. 


\subsection{Phantoms:}

Phantoms, also known as false tissues, are made from a wide range of materials to mimic certain characteristics of human tissues including thermal, optical, chemical, and mechanical properties [54, 96-111]. Phantoms have been utilized and developed to validate and invoke a number of the sensing, imaging, diagnostic, and treatment techniques. In accordance with the requirements of an application, phantoms can be liquid, solid, or gelatinous.

Phantoms in hyperthermia oncology have also played a profound effect on the development of heating and temperature monitoring devices, treatment schemes, modalities, and planning software [39, 96-114]. This is accomplished through measuring the power deposition factor, known as local specific absorption rate (SAR), of the electromagnetic energy into a tissue-equivalent material. The result geometry as a function of time and temperature are then evaluated. Performing tests on a proposed device or therapeutic approach is a crucial step for quality assurance and hence validation for clinical use in the medical field [96].

For example, exhaustive studies on polyacrymlamide (PAG) phantom have been carried out to evaluate and validate many equipment and treatment schemes in various types of hyperthermia cancer treatment techniques [97, 100, 109, 115-117]. PAG phantom has many advantages such as various polyacrymlamide phantom preparing conditions resulting in different physical properties to mimic a wide range of tissue properties and types; its ability to be used under high-temperature thermal therapies; as well as thermal lesion visualisation. An important aspect of developing a diagnostic or treatment schema 
is defining the geometry of the affected zone. For example, phantom synthesizers from PAG gel and thermochromic ink are used to acquire absolute temperature measurements along with the geometry of the thermal damage caused by radiofrequency ablation [115]. Upon heating within the range of $40{ }^{\circ} \mathrm{C}$ to $67^{\circ} \mathrm{C}$, the thermochromic ink changes its colour (Figure 2.9). The ablation effect on soft tissues such as liver can be estimated, thus entitling the development of such treatment schemes. A non-ionic surface-active agent (NiSAA) has been included in PAG phantom preparation as a temperature-sensitive indicator [116]. NiSAA also helps to recover information of the cooling processes because of its non-permanent change in colour.
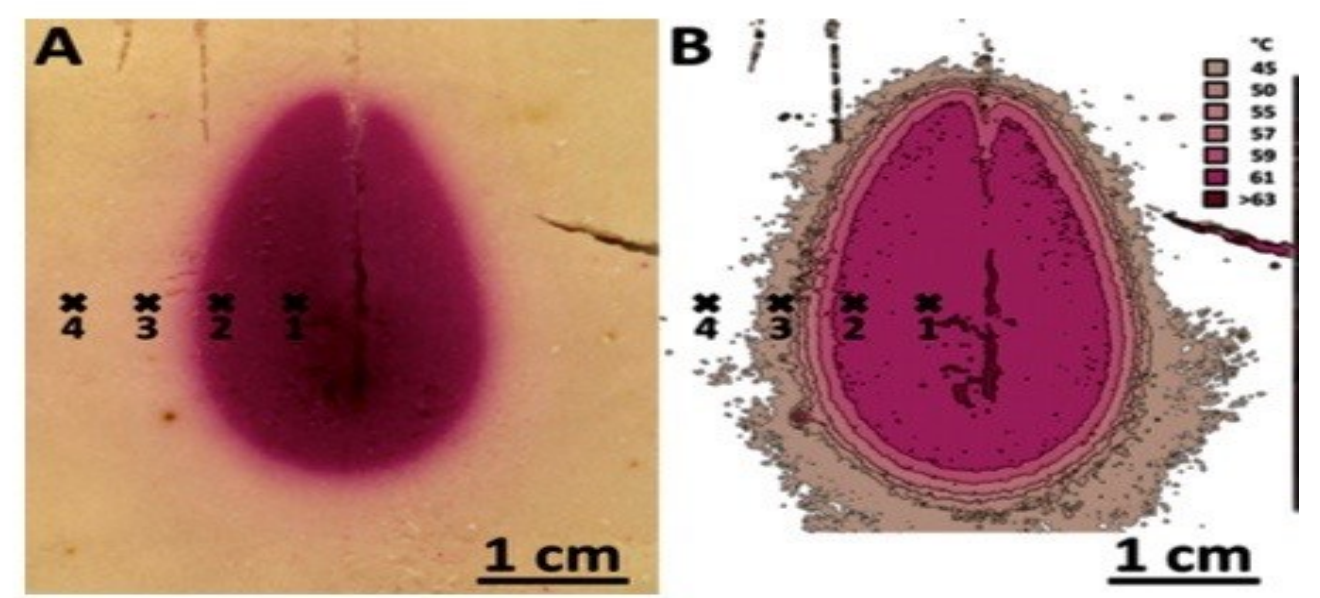

Figure 2.9. Image of TMTC phantom cross section along the RF electrode to simulate the ablation effect on soft tissues such as liver (A) and with the temperature distributions (B)[115].

Moreover, in 1991 LED phantoms were introduced to form a better understanding of the effect of RF hyperthermia therapeutic technique on some cancerous growths (Figure 2.10). The advantages of the LED phantoms are that they allows visualization of the specific absorption rate of "electric field distribution" for a false tissue undergoing hyperthermia, hence enabling the two- and three-dimensional analysis [108, 118]. Gelatine composed of peptides and proteins has been produced through collagen 


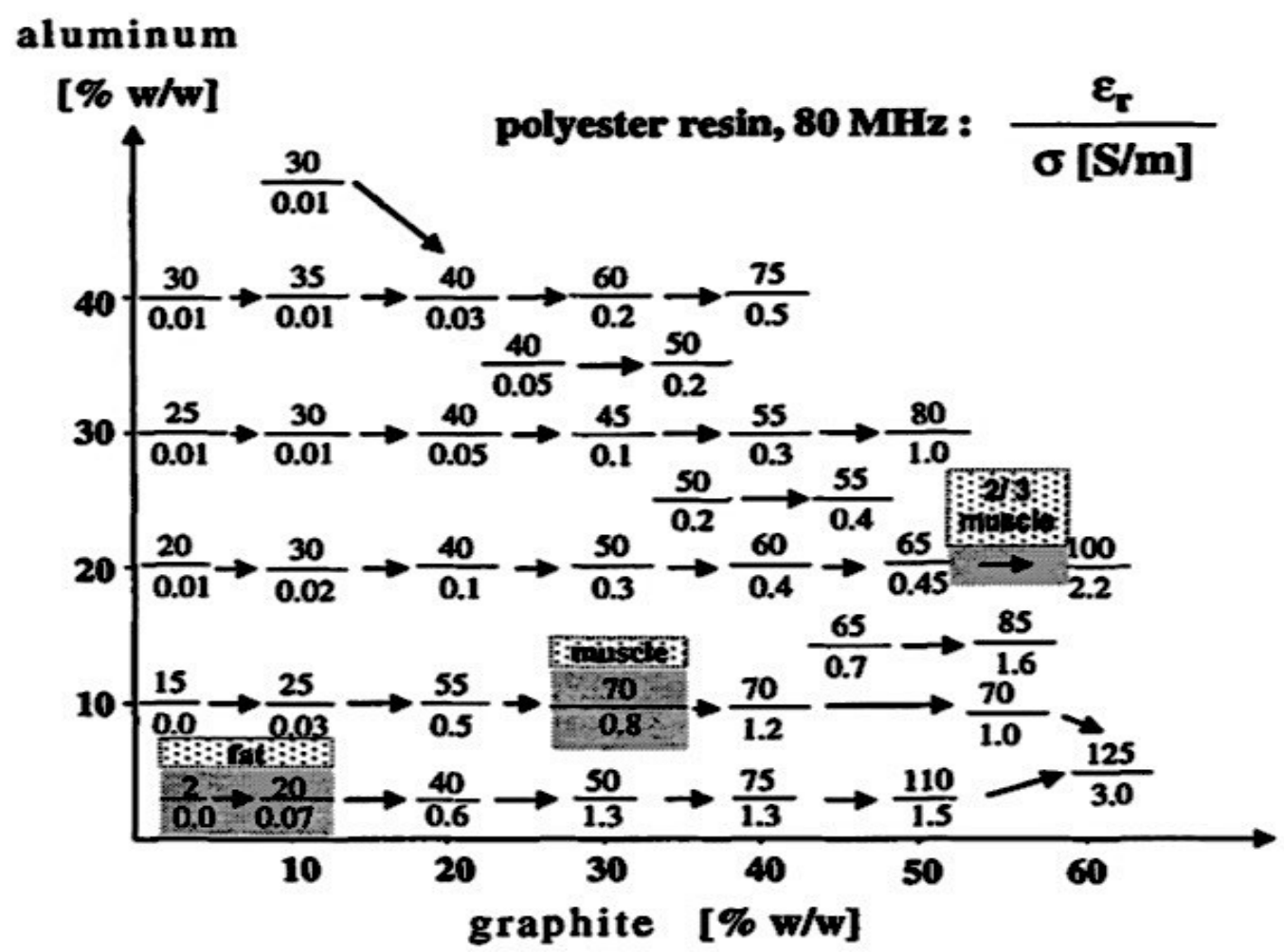

Figure 2.10. Various aluminum and graphite concentrations in polyester resin mixture produce phantoms with different electrical properties; each mixture can be used to mimic the properties of a specific tissue [118].

degradation [97, 106, 107, 119]. The degradation process is either physically, thermally, or chemically dependent on the applications. Gelatine has been utilized widely in food preparation and in the medical and pharmaceutical fields. Simple gelatine-based materials have also been adopted to mimic certain dielectric properties of a variety of high- or lowwater content tissues, and human skin depending on the type of components added to the preparation recipe. A wide range of components included in the gelatine-based phantoms such as water, oil, saline, honey syrup, and/or $\mathrm{NaCl}$ have been characterized and paralleled to simulate specific types of human tissues. Overall, the stability of gelatinebased phantoms' mechanical properties and the simplicity of their fabrication processes make them ideal phantoms to explore the microwave effect on biological tissues (Figure 2.11). 


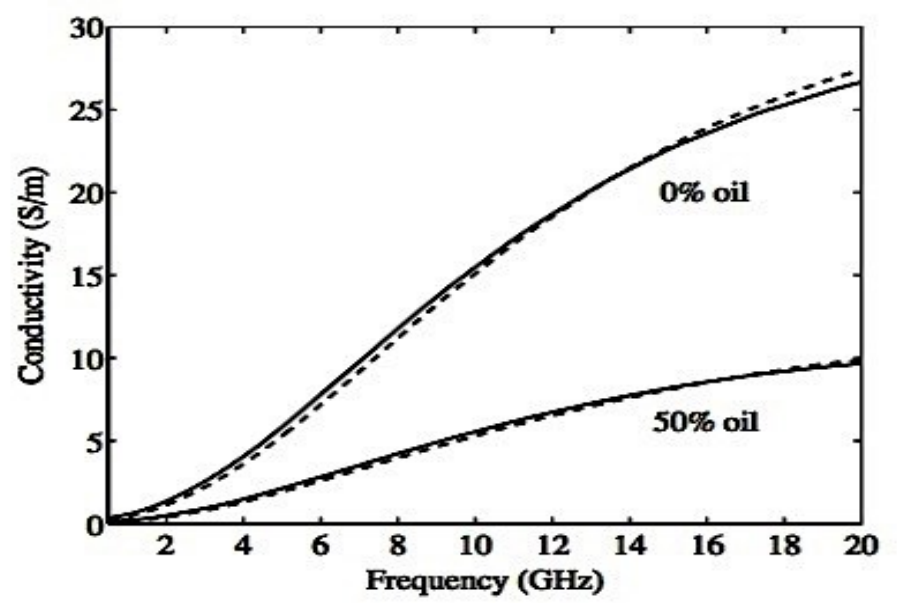

Figure 2.11. Long-time stability up to nine weeks; phantom conductivity after one week of the phantom preparation (solid lines); and after nine weeks of the phantom preparation (dashed lines) [107].

Figure 2.12 indicates a heterogeneous phantom developed by Yuan et al. to simulate the dialectical and thermal properties of a human thigh with tumor tissues [119]. The researchers intended to execute some modifications in the heterogeneous phantoms and test it with a hyperthermia therapy technique consisting of RF heating source and MR thermometry to resolve some of the issues with the available heterogeneous phantoms.

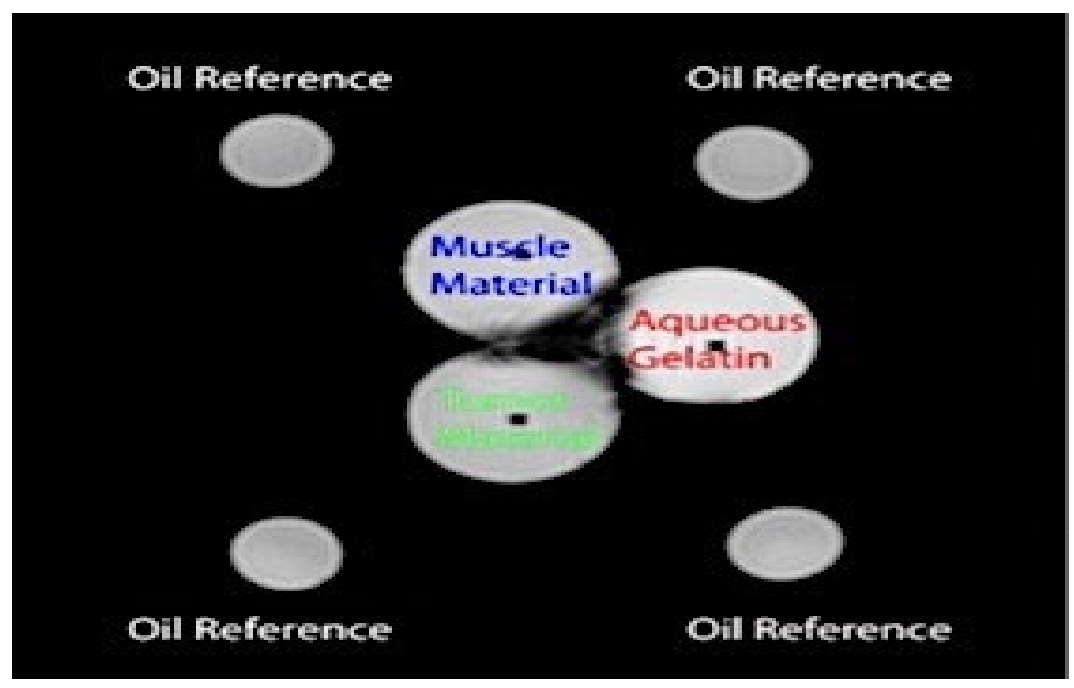

Figure 2.12. A heterogeneous phantom developed to simulate the dialectical and thermal properties of a human thigh with tumor tissues by Yuan et al [119]. 
The available heterogeneous phantoms lacked the ability to provide accurate simulation of the dielectric and thermal properties of human tissues. Through constructing the heterogeneous phantoms from the material indicated in Table 2. 4, the researchers were able to achieve a result comparable to the well-accepted results of malignant growth undergoing the same treatment technique see Table 2.5.

\begin{tabular}{llll}
\hline & \multicolumn{2}{c}{ Oil-in-gelatin dispersions } & \\
\cline { 2 - 3 } & & & Saline concentration g/1000 ml \\
Material type & Volume\% aqueous gelatin & Volume\% oil & DI water at room temperature \\
\hline Tumor & 95 & 5 & 7.5 \\
Muscle & 90 & 10 & 6.0 \\
Fat (bone marrow) & 15 & 85 & 0.24 \\
\hline
\end{tabular}

Table 2.4. Materials' percentage used to synthesis heterogeneous phantoms [119].

\begin{tabular}{lll}
\hline & $\begin{array}{l}\text { Human tissue specific heat at } 37^{\circ} \mathrm{C} \\
\left.\text { in J }(\mathrm{g} \mathrm{K})^{-1} \text { (Giering et al } 1996\right)\end{array}$ & $\begin{array}{l}\text { Phantom material specific } \\
\text { heat at } 37^{\circ} \mathrm{C} \text { in J }(\mathrm{g} \mathrm{K})^{-1}\end{array}$ \\
\hline Tumor & $3.51-3.80$ & 3.71 \\
Muscle & 3.55 & 3.48 \\
Fat & $2.22-2.43$ & 2.17 \\
Bone cortex & 1.3 & 1.24 \\
\hline
\end{tabular}

Table 2.5. Heat in tissues vs heat in phantoms [119].

Egg white phantoms have been used for thermal lesion visualisation to mimic the thermal conductivity of tumor tissues undergoing various types of hyperthermia cancer treatments $[111-112,120]$. Similar to human blood which fills tissues, water is the major constituent (in order of $90 \%$ ) of the hen egg white dissolved into proteins. 
Thus, the approximate thermal conductivity and heat capacity of some human tissues can be mimicked through using fresh egg white (Table 2.6). The egg white phantom is utilized either individually or in combination with other materials as a temperature sensitive indicator.

\begin{tabular}{lccccccc}
\hline \hline & \multicolumn{3}{c}{ Pig/cow/human blood } & & \multicolumn{3}{c}{ Egg white } \\
\cline { 2 - 5 } \cline { 6 - 8 } & $10 \mathrm{~s}$ & $30 \mathrm{~s}$ & $10 \mathrm{~min}$ & & $10 \mathrm{~s}$ & $30 \mathrm{~s}$ & $10 \mathrm{~min}$ \\
\hline $55^{\circ} \mathrm{C}$ & 1 & 1 & 1 & & 1 & 1 & 1 \\
$65^{\circ} \mathrm{C}$ & 1 & 1 & 2 & & 2 & 2 & 2 \\
$75^{\circ} \mathrm{C}$ & 2 & $2 / 3$ & 3 & & 2 & 3 & 3 \\
$85^{\circ} \mathrm{C}$ & 2 & 3 & 3 & & 3 & 4 & 4 \\
$95^{\circ} \mathrm{C}$ & 3 & 3 & 3 & & 4 & 4 & 4 \\
Blood & 1. Original red blood 2. Red mucus/viscous liquid 3. Chocolate solid gel \\
Egg white & 1. Original clear liquid 2. White liquid 3. White mucus/viscous liquid 4. White solid gel \\
\hline \hline
\end{tabular}

Table 2.6. Approximation of heat capacity of pig, cow, and human blood vs egg white [112].

Figure 2.13 shows the effective temperature needed to cause irreversible visible lesion [120]. This phantom is synthesized from polyacrylamide gel and fresh hen egg white. The gel mixture in this study is needed to characterize the acoustic properties of the high intensity focused ultrasound traveling in such a phantom, in addition to acquiring the induced thermal effect of this therapeutic technique. In microwave and high intensity focused ultrasound hyperthermia, the egg white manifests its ability to be used as an alternative available temperature sensitive indicator to a number of indicators such as bovine serum albumin (BSA) [120]. 


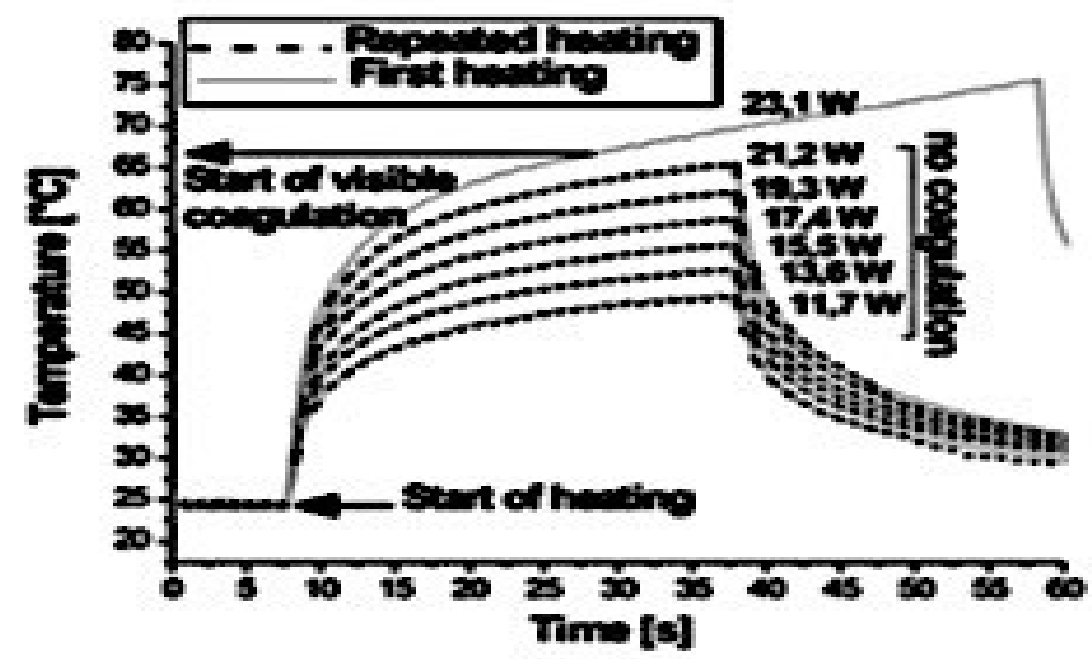

Figure 2.13. Effective temperature needed to cause irreversible visible lesion [120].

A number of phantoms are commercially available including urethane-rubber based from ATS labs, Zerdine from CIRS Inc., and condensed milk from Gammex-RMI [97, 121].

There are similarities between certain mechanical and physical properties of these phantoms and human tissues. However, some limitations exist in the available data about their performance; for example, exposure at various frequencies to different temperatures. 


\section{Chapter 3: Grating-Based Fiber Optic Sensors}

A revolution in fiber fabrication techniques along with the various appealing characteristics of FBGs technology have given birth to diffractive gratings with various structures including slanted or tilted fiber Bragg gratings (TFBGs), chirped fiber Bragg gratings (CFBGs), and chirped tilted fiber Bragg gratings (CTFBGs). In addition to such configurations, FBG within itself can be written with different grating pitches to obtain high order gratings or long fiber Bragg gratings (LFBGs). Besides the Bragg-guided mode resonance, each of these structures suppresses, fosters, and/or enhances special mode coupling mechanisms. Considering the topic of this thesis, our discussion is restricted to TFBGs and the thermal stability of first- and third- order fs-FBGs. The thermal stability of the diffractive gratings is a further essential point to the work in this thesis as the human tissues are usually exposed to extreme conditions in localized thermal therapy.

First the sensing principle and the fabrication methods of FBGs are briefly described. These sections are followed by a description of the additional optical properties of TFBGs and thermal stability of first- and third- order fs-FBGs.

\section{1 Introduction to Fiber Optic:}

A pronounced revival in technological advancements has been identified in the literature since the discovery of fiber optic technology in the early 1970s, especially in the telecommunication industry which enables the transmission of information over large distances [131-135, 137-139]. Sensing technology has been influenced by the revolution of fiber optic technology. Sensor-based fiber optics have built their position in the 
sensing field on account of many factors including their passivity, reliability, long life expectancy of "more than 20 years", stability with "no need for calibration", and small size accompanied by low cost.

Three major configurations, interferometric sensors, distributed sensors, and gratingbased sensors represent the fiber optic sensors (FOS) field [83, 85, 87, 131-132, 140]. Fiber Bragg Grating is the most fully developed technique in grating-based sensors technology. FBG sensors have already proven their industrial utilization, for example through their commercialization by many companies such as HBM. In addition, owing to the development of new materials and improvement in fabrication techniques, FBGs have great potential in a variety of fields [141-144]. For example, utilization of a special type of coating on the fiber enables the measurement of DC and AC magnetic fields, ultrasonic imaging, dynamic strain measurements, and so on.

A FBGs sensing method differs from other kinds of fiber-optic sensors $[6,83,85,87$, $131-136,138,145]$. The sensing method of most fiber-optic sensors is based on monitoring the modulation of light properties such as phase or intensity which are susceptible to intensity changes received by the detector. Many factors may cause intensity losses including losses at fiber couplers, fluctuation in the source power, or fiber bending. In contrast, the change in the Bragg wavelength is not reliant on the overall light levels. Thus, the intrinsic method is more desirable to be utilized over intensity-based sensing method for a wide range of applications.

One factor which must be taken into account is the influence of a quasi-static variation of the strain and temperature on measurements and vice versa as both measures cause a shift 
in Bragg wavelength. With the FBGs temperature sensor, this cross-sensitivity problem is avoidable by isolating the sensors form strain. On the other hand, the temperature effect on FBGs strain sensors is difficult to avoid. The most common solution relies on colocating two signals by hybridizing two different types of gratings, such as FBG and a long period fiber grating (LPFG) written in an adjacent section of fiber.

In addition to robustness to noise and power fluctuation, a number of gratings can be embedded into a single fiber for use as a multiplexed sensor web where various parameters are measured such as different stresses or temperatures at different locations [89, 131-136]. Further attractive advantage consists of the real time feedback obtained from FBG-based sensor to assess and monitor the sensing environment. Actually, these features cause FBGs sensor to stand out among other FOS sensing technologies in numbers of field including the medical field. However, the equipment cost is high in order to prevent the decrease of the multiplexed sensors' performances in the areas of resolution and accuracy.

Advancements in the field of endoscopic imaging (imaging sensors) comprise the most significant impact of the fiber optic technology in the medical field and the most developed technique [87]. Alternative uses of FOS in the biomedical community include physical parameter sensors (temperature, pressure, etc.) and biochemical sensors ( $\mathrm{pH}$, species constriction, etc.). Unlike biochemical sensors, physical parameter sensors are currently more developed and available in the market $[146,147]$. By way of illustration, FBG-based temperature sensors have been considered in the development of intelligent clothing [146]. Intelligent clothing is a type of regular human clothing which has been equipped with sensors to monitor, for example, human body temperature, and to provide 
data in real time. This type of technology could have a significant impact on the early detection of diseases accompanied by fever resulting from non-infectious diseases including tumors and metabolic diseases or from infectious diseases such as influenza. The early detection of changes in human body temperature is vital, especially when timing can make a considerable difference in the treatment and recovery process in young children and older people.

\section{2 Sensing Principle of FBGs:}

The basic sensing principle of FBGs is based on a periodic and permanent perturbation of refractive index within the core of an optical fiber which has a special resonance known as Bragg wavelength resonance (Figure 3.1) [131-135, 148]. The behaviour of such gratings is similar to that of a multilayer mirror, meaning that light carrying specific

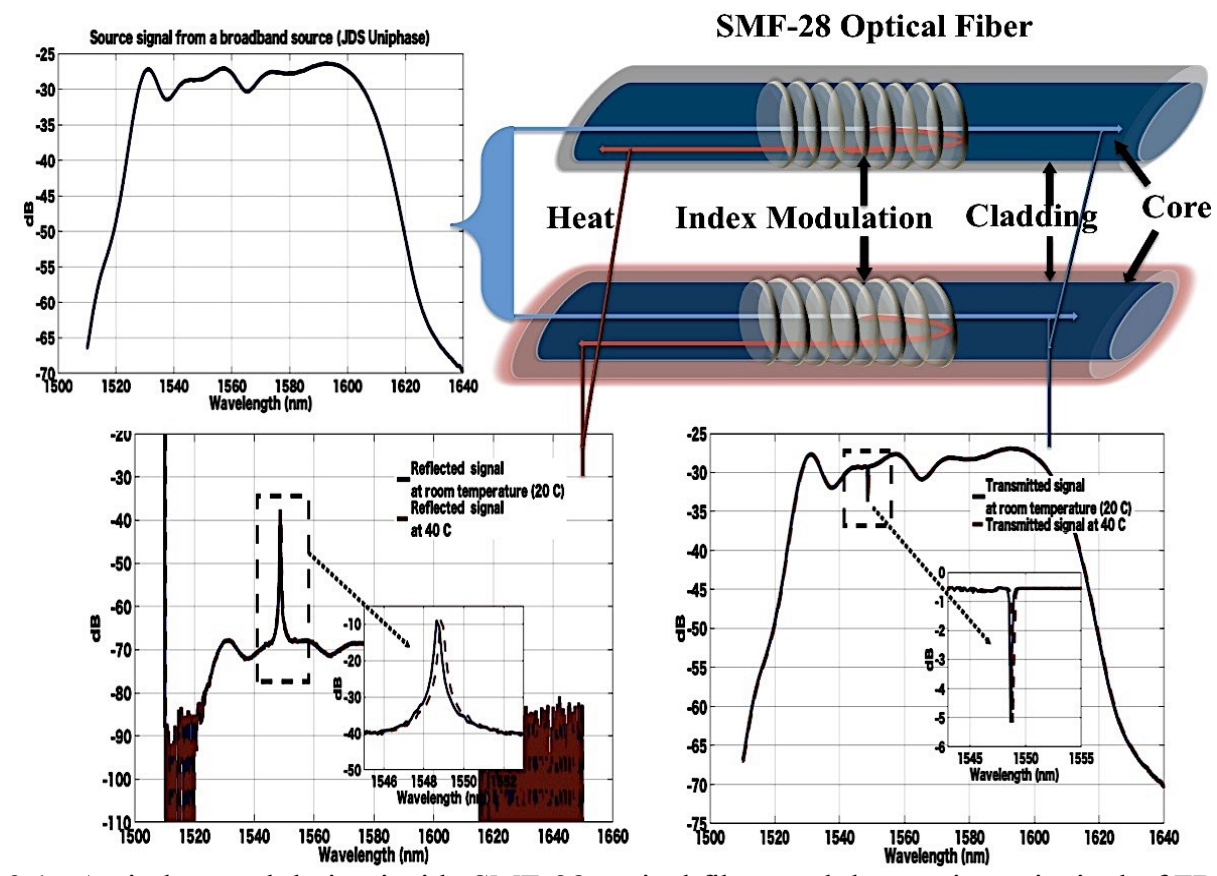

Figure 3.1. An index modulation inside SMF-28 optical fiber, and the sensing principal of FBGs based temperature sensors with transmissive or reflective detection options.

narrow wavelengths is reflected from a series of reflected planes with the phase fronts which are perpendicular to the fiber's longitudinal axis, followed by transmitting all other 
wavelengths of the light. The central reflected wavelength matches the following equation:

$$
\lambda_{B}=2 n_{e f f}^{c o} \wedge
$$

where $\lambda_{B}$, and $n_{e f f}^{c o}$ refer to the resonant Bragg wavelength and the effective core refractive index respectively. $\wedge_{g}$ is the grating spacing which can be expressed as $\wedge=\frac{\wedge g}{\cos \xi}$ in the presence of a tilt in the fringes of the gratings $\xi$. From this straightforward description based on Bragg diffraction theory, the fundamental coupled mode theory can be implemented to monitor the above parameters in practical applications. The reflectivity to Bragg wavelength of such structure is usually expressed as follows:

$$
R(l, \lambda)=\frac{k^{2} \sinh ^{2}(\rho l)}{\Delta \beta^{2} \sinh ^{2}(\rho l)+\rho^{2} \cosh ^{2}(\rho l)}
$$

where $k, l$, and $\Delta \beta$ are the coupling coefficient, the grating length, and the detuning wave vector, respectively. $\rho$ is equal to $\sqrt{k^{2}-\Delta \beta^{2}}$.

Therefore, the reflectivity, bandwidth, or coupling efficiency between the forward propagating and counter-propagating modes of the grating is proportional to the length (l) of the grating and to the strength $(\Delta n)$ and the periodicity $(\Lambda)$ of the induced index of refraction [131-135]. The values of these parameters, which can be designed as needed, usually range between $200 \mathrm{~nm}$ to $1 \mu \mathrm{m}$ for $\wedge$, a few millimetres to a few centimetres for length $(l)$, and $10^{-5}$ to $10^{-2}$ for strength $(\Delta n)$. 
A weak grating is more favourable for use in sensing applications because of the required precise determination in the change of the Bragg wavelength from the obtained narrow spectrum [87, 131-135]. A reflectivity of $95 \%$ or less gives a narrow reflected spectrum in comparison to the almost $100 \%$ reflectivity from the strong grating. In contrast, extremely short grating is more desirable in the application of FBG-based strain sensor due to deformation of the Bragg signal caused by the effect of micro-strains [149]. This ability to tolerate the resonant Bragg wavelength of FBGs allows for their widespread utilization in sensing applications.

FBG is intrinsic sensor meaning that the device itself is the sensing element $[28,29,131$ 135]. For example, a shift in the Bragg wavelength resonance which corresponds to temperature change $(\Delta T)$ around the sensor results from the changes in the effective refractive index and the gratings period (Figure 3.1). Further, this device is sensitive to any mechanical change in the medium. In this case, the shift in Bragg resonant results from the changes in the gratings period and in the inducement of the photoelastic change in the effective reflective index due to applying axial strain $(\epsilon)$ on the fiber. The thermal and mechanical sensitivities of this device range from $6.8 \mathrm{pm} / \mathrm{C}$ to $13 \mathrm{pm} / \mathrm{C}$, and $0.64 \mathrm{pm} / \mu \varepsilon$ to $1.2 \mathrm{pm} / \mu \varepsilon$, where $\mu \varepsilon$ denotes to the microstrain, respectively [28, 29, $131,133]$.

\section{2. 1 Sensing Principle of TFBGs:}

TFBGs possess the same sensing principle with more complex spectral behaviour because of the permitted coupling between modes of dissimilar azimuthal symmetry (Figure 3.2) [152-161]. In other words, with the presence of the inclination angle between the grating plane and the fiber cross-section, cladding mode resonances are observed 


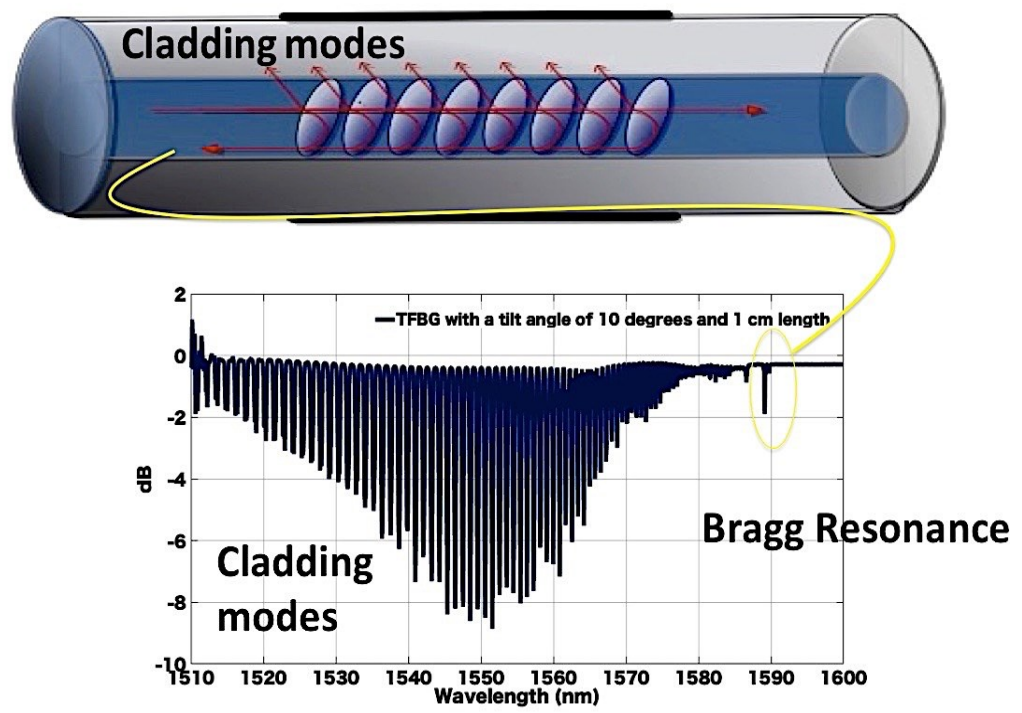

Figure 3.2. Bragg guided mode and cladding mode resonances in the transmission spectrum of the TFBG because of the presence of the inclination angle.

alongside the Bragg guided mode in the transmission spectrum of the TFBGs:

$$
\lambda_{C l, i}=\left(n_{e f f}^{c o}+n_{e f f, i}^{c l}\right) \wedge
$$

Where $n_{e f f, i}^{c l}$ is the effective refractive index of the $i$ th cladding mode. Accordingly, the cause of this series of discrete cladding modes is based on mode couplings between the diffracted light of the forward propagating core mode and backward-propagating cladding modes which have been allowed by writing this grating with some degree of an inclination angle. Also, radiation modes have been observed in such configurations resulting from coupling between forward-propagating high order cladding modes into backward-propagating radiation modes, hence leaking into the environment.

Coupled mode theory is a fundamental technique which has also been implemented to analyze the TFBG spectra theoretically. This old technique has been adopted and 
modified by Erdogan et al. to tackle the TFBG spectra $[153,159,161,162]$. Some researchers have also implemented the volume current method to evaluate the spectral characteristics of TFBGs including the phase-matching condition, wavelength dependence, and angular distribution of TFBG [163-165]. The volume current method is considered by the literature to be a fundamental approach alongside the well-established coupled mode theory. This method was introduced in 2001 by Li et al. to investigate spectral characteristics of TFBG. A number of spectral characteristics of TFBG have been described by using the antenna theory whether based on the far or near field. Through implementing this method along with the coupled mode theory, the dependency of the radiation mode on the fiber orientation has been proven [166].

In addition to the presence of Bragg guided resonance, the cladding and radiation modes are becoming the rationale of the research engine both academically and within the industry of developing multi-dimensional photonic devices on account of the following $[154,165-171]:$

1) Through manipulating the gratings' tilt angle, the width of the cladding mode resonance can be controlled and monitored. Hence, the separation wavelengths region between the utmost radiation's wavelength and the Bragg-guided wavelength can be monitored, and hence TFBG can function by filtering and wavelength division multiplexing (WDM). For example, mode conversion and demultiplexing can be obtained by weakly tilted-FBG as demonstrated by Ya G et al. Mode conversion with up to $99.5 \%$ efficiency from $\mathrm{LP}_{01}$ to $\mathrm{LP}_{11}$ is achievable when using TFBG with a tilt angle of 1.6 degree [172].

2) The significant influence of the cladding effective refractive index through the 
variation in the environmental refractive index make TFBG suitable for use as a temperature-insensitive quasi-distribution TFBG refractometer, optical spectrometer label-free biosensor, and relative humidity sensor.

3) Cladding modes and the core mode sensitivity to temperature changes around the fiber are the same, yet they possess different responses to strain and refractive index variations. The sensitivity of the cladding mode resonances differ according to the order of the mode, as each mode is characterized by its own effective refractive index. This means that higher order modes have greater sensitivity than lower order modes to interruptions from the surrounding environment. Therefore, highly accurate measurements with an ability to eliminate the temperature cross sensitivity through measuring the relative cladding wavelengths' shift to the core wavelength enable TFBG-based sensors to serve as a low cost multi-functional device for simultaneous measurements of strain and temperature, discrimination of bending and temperature, and refractive index and temperature. Note that this sensitivity of TFBG modes is present when the grating is embedded into, for example, a SMF-28 fiber. On the other hand, reaction of TFBG modes differs when the grating is written in, for example, a plain-wave composite material.

4) Radiation modes of TFBG propagate along different directions for different wavelengths [173-175], thereby making it suitable for TFBG-based wavelength interrogation through side radiation, and optical spectrometers.

5) TFBGs with large angles $\left(\theta_{B}>15^{0}\right)$ are sensitive to the polarization state of incident light; hence, they can be used as polarimeters and twist sensors. 
Furthermore, due to the promising potential of TFBG-based sensors, TFBG sensors have been implemented in various other applications $[87,83,131-135,176]$. Huy et al. have illustrated the tilted angle role in optimizing the gratings' sensitivity, written in PCF, to the variation of the ambient refractive index [170]. Compared to conventional FBGs, the noticeable improvement in the performance of the developed device is a result of optimizing the mode coupling between the core-guided mode and the higher order cladding modes. As a further example, researchers have devoted a great deal of interest in investigation of plasmonic resonance in TFBG-based biosensors $[152,154]$. This may entail the investigation of a wide range of biochemical processes including molecular interactions, concentration, and dynamic behaviour.

The degree of the tilt angle and the strength and the length of the refractive index modulation determine the coupling efficiency, the bandwidth of the cladding mode resonances, and hence the device sensitivity $[152,154-157,160,166]$. Generally, as the tilted angle of the gratings increases, the TFBG transmission spectrum extends to the short-wavelength side away from the Bragg wavelength which results in more coupling between the higher order cladding modes and the radiation modes. To obtain objective appraisal of an application, these parameters can be manipulated.

Besides the three factors described above, other parameters may also have an impact on the device performance such as the type of fiber and the size of the cladding region [152$154,177,178]$. For example, one proposed approach toward enhancing TFBG sensitivity is by reducing the cladding diameter through the hydrofluoric (HF) acid etching method. As the cladding diameter is reduced, more cladding mode resonances leak to the sensing environment and thereby improve the sensitivity of the cladding mode resonance to the 
changes in the surrounding environment. The device's sensitivity to any mechanical interruption remains constant.

\section{2. 2 Sensing Principle of High Order FBGs:}

High order fiber Bragg gratings are diffractive gratings written with a grating pitch of more than one micrometer [136, 161-184]. Besides the direct coupling between the forward-backward modes to obtain the Bragg resonance, a further distinctive feature of the high-order gratings is the indirect coupling between the two contra-propagating guided lights resulting in the excitation of the radiation waves' "radiation modes" [181]. In other words, increasing the grating period causes enhancement of the radiation coupling modes. The effects of the length $(l)$ of the grating and the strength $(\Delta n)$ of the index modulation on the refractive power are similar to first order grating, described above. However, certain optical characteristics of high order gratings differ from those of first order devices. For example, high order FBGs possess a weaker and narrower Bragg resonance. The radiation modes are important in a number of applications including grating-outcoupled surface-emitting laser, laser pumping sensors, and free space communications $[183,184]$. Researchers have found that radiation power of $0.012 \mathrm{~mW}$ from a second-order fiber grating can be emitted to free space through the cladding region (Figure 3.3) [182].

\section{3 Thermal Stability of FBGs Sensors:}

The well-known FBGs-based sensors are often called Type I gratings. Type I gratings have low thermal stability manifested by decreasing the sensing signal associated with drift in Bragg wavelength until it is erased completely at an approximate temperature of 


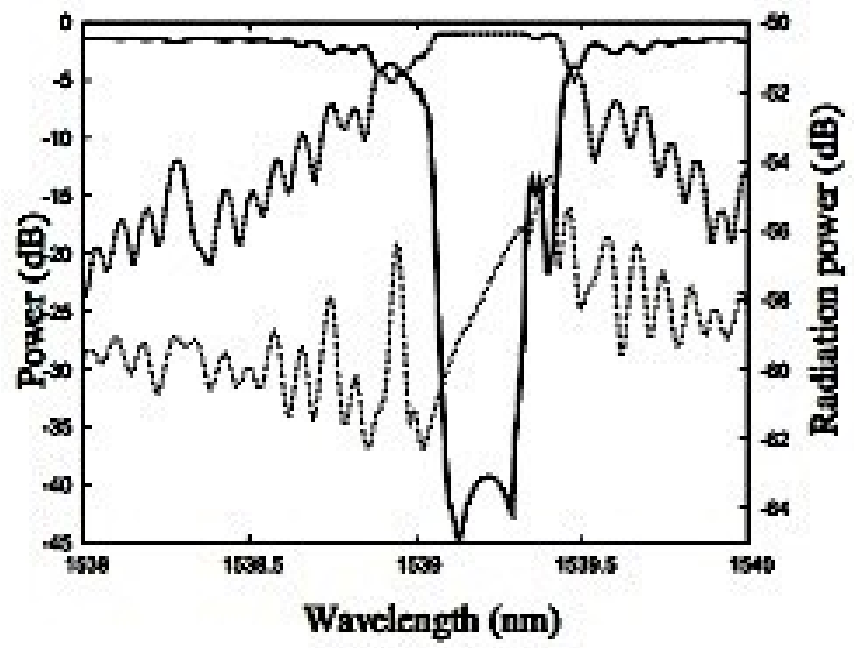

Figure 3.3. An example of the transmission, reflection, and radiation spectra of second-order FBGs [182].

$500{ }^{\circ} \mathrm{C}$. In contrast to Type I gratings, Type II gratings and RFBGs have shown thermal stability up to $1000^{\circ} \mathrm{C}$, a characteristic which makes them ideal for use in harsh environments [132-135, 185]. As opposed to Type I gratings, Type II gratings do not have many sub-class types because their spectra are not as easily tailored. This limitation associated with the origin of Type II index modulation results from writing the device at a high power above the damage threshold of glass; hence, they are usually referred to as damaged gratings.

Regenerated gratings are created after Type I gratings are erased through a thermal regeneration process [186-191, 195-200]. Different thermal stabilities of the regenerated gratings have been reported depending on the writing conditions and the type of the fiber. The highest reported thermally stable RFBGs works at $1400{ }^{\circ} \mathrm{C}$ written in erbium-doped yttrium stabilized zirconia-calcium-alumina-phosphor silica (Er-YZCAPS) glass [192]. Despite the writing conditions and the type of the fiber utilized to write the regenerated gratings, most of the reported RFBGs have the same good controllable spectral response 
which is comparable to type I gratings; therefore, regenerated gratings ideal for narrow band or multiplexed sensors. On the other hand, as a consequence of Type II damage growth mechanism, they exhibit significant out of band losses. This characteristic limits their use as a multiplexed sensor.

Accordingly, a great deal of research has been carried out to comprehend [135, 186-194], and optimize the regenerated gratings, and hence enable their use in industrial applications such as coal gasification, turbines and aluminum smelting. Reasons behind the thermal stability of RFBGs are still not fully understood. However, the influence of various parameters on parts of RFBGs optical characteristics are being comprehensively illustrated in the literature such as the presence of hydrogen, the fiber type, the fiber doping type, and the laser type used in the gratings inscription [186-191, 195-200].

The main issue of the regenerated gratings is the final small index change limited their use in practical applications. In 2009, Smelser et al. reported that higher order gratings have the ability to retain a larger portion of their initial index modulation with high stability at $1000{ }^{\circ} \mathrm{C}$. The high-order gratings were written using a femtosecond laser in a hydrogen-loaded SMF-28 [70]. Further, these gratings did not generate during the annealing process, and were written with a laser energy that was lower than the threshold damage energy of the glass. Hence, these high order gratings are not regenerated gratings, nor are they Type II gratings, yet they are stable at $1000^{\circ} \mathrm{C}$. High order gratings' thermal stability is correlated to the large pitch of the gratings. 


\section{4 Fabrication Techniques:}

The discovery of FBG dates back to the 1970s when researchers at the Communication Research Centre Canada first noticed the formation of a locally periodic modification in the core of a standard telecommunication optical fiber upon exposure to a single mode argon ion laser operating at $488 \mathrm{~nm}[29,131-135]$. However, further interest in this discovery was staved off because the reflected wavelength by the grating was the same wavelength of the used argon ion laser beam, and no significant use to the device was noticed.

The introduction of FBG in the sensing field began in 1989 [65-69, 131-135]. The ability to inscribe gratings in the fiber core is based on the photosensitivity of germanium oxide dopants in the core of telecommunication optical fiber to the exposure wavelength. Since then, a number of techniques including standing wave, holographic, phase mask, and point-by-point writing have been applied to manufacture gratings.

The holographic and phase mask methods are suitable for practical applications because of their ability to produce gratings with good performance and a capability of being implemented for mass production [131-135, 148, 201-204]. Although these methods have different fabrication techniques with various features, the writing approach of both methods is based on an optical interferometer pattern.

\section{4. 1 Holographic Method:}

The holographic writing technique had been reported by Meltz et al [131-135]. First, a beam splitter is utilized to divide a UV beam into two parts. These two beams are then collimated and interfered at the fiber. The Bragg resonant resulted from the created 
sinusoidal refractive index pattern in the fiber core depends on the UV-source wavelength and on a mutual angle as follows:

$$
\lambda_{B r a g g}=\frac{n_{e f f} \lambda_{u v}}{n_{u v} \sin (\theta / 2)}
$$

where $\lambda_{\text {Bragg }}$ and $\lambda_{u v}$ refer to the Bragg reflection wavelength and the writing wavelength, $n_{e f f}$ and $n_{u v}$ are the effective mode index and the refractive index of silica in the $\mathrm{UV}$, and $\theta$ is the mutual angle of the UV-beams. An essential requirement of this technique is the utilization of a high coherent laser source, like frequency doubled $\mathrm{Ar}^{+}$. Such a laser is needed to avoid changes in the quality of the interferogram which may be caused from the light low spatial coherence, light traveling along the path in air, or by some mechanical vibration in the system. Therefore, this method is mostly applied for short gratings written by a single-pulse.

The most attractive merit in the holographic writing technique is the flexibility in writing FBGs of an adjustable period, unlike the case with the phase mask. As a result, the drawtower method, or the direct writing technique, has adopted this writing technique with some modulation and pushed this sensor toward industrial applications [135, 148].

As can be seen in Figure 3.4, the gratings are written at the same time as the fiber get fabricated $[135,148]$. As a result, arrays of grating are produced with high intrinsic strength and optical properties. Further, gratings with variable reflection wavelengths and with small distances between adjacent gratings of approximately $5 \mathrm{~mm}$ can be generated. It is worth to mention that even though this technique was initially developed to produce Type II gratings, because of the applied high-powered laser pulses, standard FBGs are 
much more commonly used in the industry.

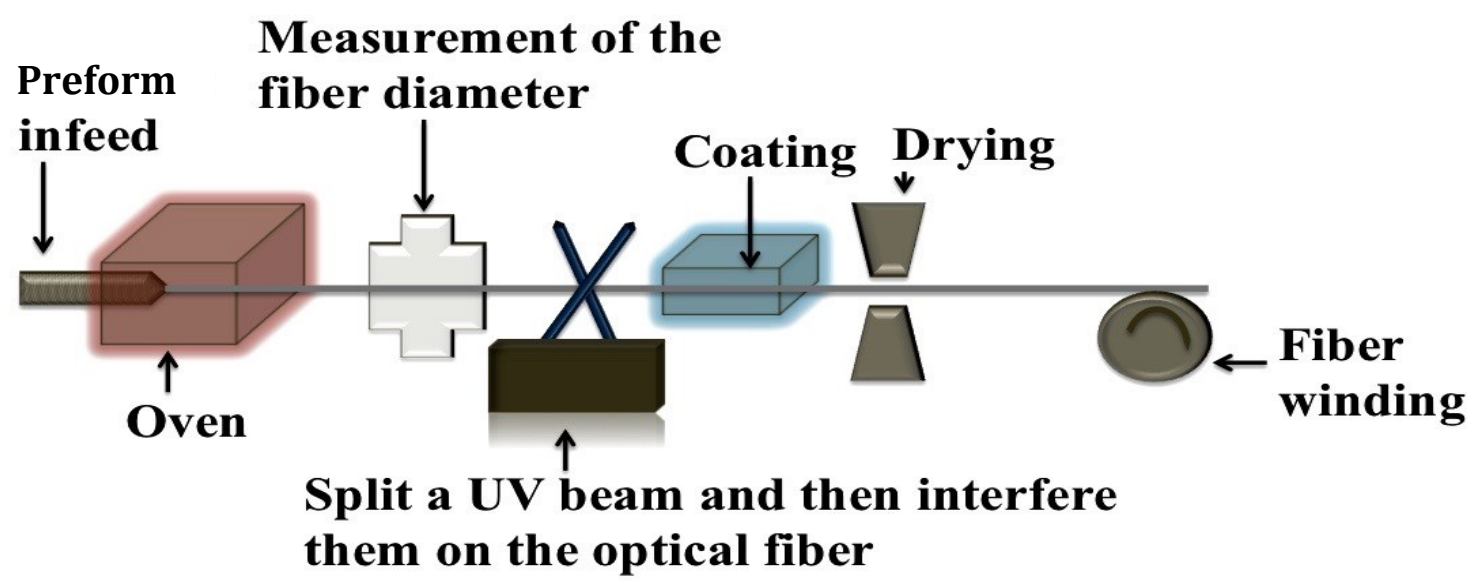

Figure 3.4. The draw-tower setup; the gratings are written at the same time as the fiber get fabricated.

TFBGs with distinguishable high performance can be obtained from the holographic writing technique [154]. However, the main limitation in this technique is the dependence of the grating period on the tilt angle of the gratings. On the other hand, no dependence is observed when using the phase mask inscription approach to fabricate the TFBGs. Hence, the phase mask inscription approach is more favourable than the holographic technique on account of its simplicity and flexibility.

\section{4. 2 Phase Mask Method:}

The development of FBGs in sensing applications is derived from the phase mask fabrication technique [65-69, 131-135, 201-204]. This technique simplifies the laboratory fabrication of the device to explore its properties; moreover, it offers high volume and low cost for mass production. The phase mask technique works by interfering diffractive orders of light $(m=0, \pm 1, \pm 2 \ldots)$ to create the sinusoidal intensity pattern in the fiber core which is generated by the diffractive phase mask. In this case, the period of the 
gratings does not depend on the source wavelength. Thus, the Bragg wavelength can be directly calculated from Bragg's law:

$$
\lambda_{\text {Bragg }}=2 n_{e f f} \wedge_{p m}
$$

where $\Lambda_{p m}$ is the period of the phase mask, given by:

$$
\Lambda_{p m}=\frac{m \lambda_{U V}}{\sin \left(\frac{\theta_{m}}{2}\right)-\sin \left(\theta_{i}\right)}
$$

where $\frac{\theta_{m}}{2}$ is the angle of the diffracted order, and $\theta_{i}$ is the angle of the incident UV beam. Another attractive feature of this technique is that the use of the phase mask enables grating inscription through low spatial and temporal coherence sources, unlike the holographic method, due to improvement in writing set-up alignment [131-135]. However, the gratings pitch is fixed by the amplitude of the phase mask patterns $\Lambda_{p m}$. The fact that various masks require different gratings is the main downside of utilizing the phase mask approach.

\section{4. 2. a High Order FBGs:}

Producing gratings with a specific strength from a phase mask with larger $\Lambda_{p m}$ "such as mask for second or third order FBGs" requires longer exposure time and/or higher exposure energy when compared to a phase mask with smaller $\Lambda_{p m}$. The energy carried by \pm 1 orders light diffracted from a phase mask with $\wedge_{p m}$ equal to one $\mu m$ is more than $35 \%$ of the light incident on the phase mask. This percentage is much less when the period of the phase mask $\wedge_{p m}$ increases to three micrometers, for example, in order to 
obtain third-order FBGs. Thus, femtosecond laser source is more favorable to be applied in high order FBGs fabrication than UV laser sources.

\section{4. 2. b TFBGs:}

The phase mask approach can also be applied to inscribe gratings with tilt angle [152, 154, 178, 204]. Two methods have reportedly been used to write TFBG: 1) rotating the phase mask and the fiber; and 2) rotating the phase mask around the axis of the laser beam while keeping the fiber and the phase mask perpendicular to the laser (Figure 3.5).

Each technique has advantages and disadvantages. While ease of use is the most important feature provided by the latter technique, we use the former method to write gratings in our photonic lab at Carleton University [152]. This method allows a flatter fringe pattern inside the fiber with very strong and narrow cladding mode resonances due to distorting the beams diffracted by the mask raised from rotating the fiber with respect to the phase mask.

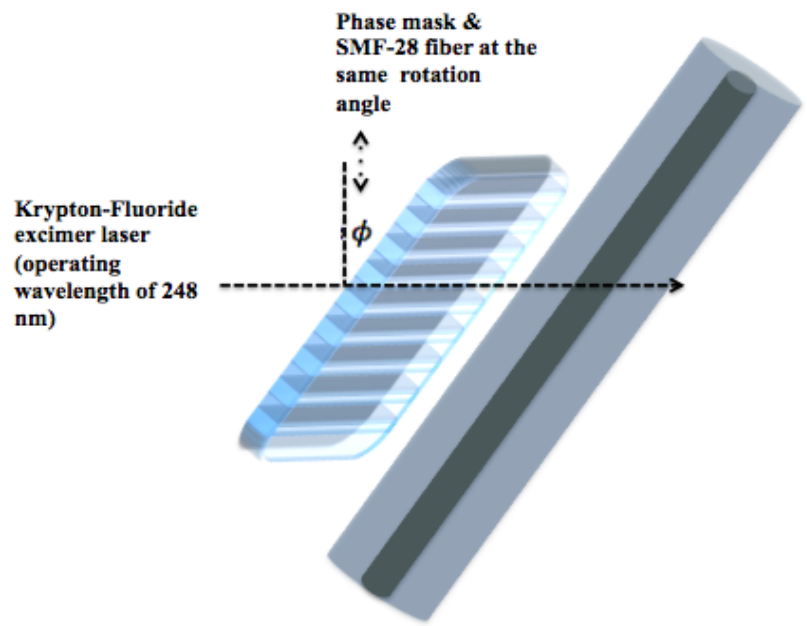

Figure 3.5. The phase mask technique to fabricate TFBGs. 
Depending on interference between plus- and minus-one orders on the fiber, for example, which is placed behind the phase mask at an azimuthal tilt angle $\theta$ with respect to the phase mask rotation angle $\phi[152,154,178,201-204]$, the tilt angle of the inscribed gratings $\theta_{B}$ can be obtained by controlling $\phi$. In this case, the utmost achievable $\theta_{B}$ of the gratings is restricted to Snell's law. On the other hand, if large tilted angle $\theta_{B}$ is desired, the handling of both angles of the fiber and the phase mask must be considered. For further details about employing the phase mask technique in writing complex Bragg grating structures, please refer to a recent comprehensive study conducted by Melo et al. [202].

Depending on which laser is used, the fabrication conditions, as well as the optical fiber type, FBGs have been divided into several types [131-135, 185]: Type I, Type II, and regenerated gratings (RFBGs). Different mechanisms responsible for the formation of FBG types result in disparity among the physical properties, including thermal stability. 


\section{Chapter 4: Materials and Methods}

This part of the thesis consists of two main experimental systems: 1) the heating system to induce hyperthermia in cells and tissue-like materials; and 2) the annealing system to evaluate the thermal stability of fs-FBGs. For the heating systems, we describe the writing conditions of TFBGs, the heating setup, the coating and packaging materials, and the heated target materials including three cell lines, gelatine- and egg white-based phantom, and porcine liver. For the annealing system, we illustrate the writing conditions of first- and third-order fs-FBGs and the annealing setups.

\section{1 Heating System to Induce Hyperthermia:}

\section{1. 1 TFBG-based Heater Fabrication Conditions:}

One-centimetre-long TFBGs were inscribed in Corning SMF-28 single mode optical fiber of the kind used for telecommunications at near infrared wavelengths. The fiber was hydrogen-loaded by diffusion at room temperature for 10 days at $16 \mathrm{MPa}$ in a pressurized tube in order to increase its photosensitivity. Prior to writing the grating, the protective polymer jacket of the fiber was removed over a few centimetres to expose the glass surface of the fiber cladding. This section of fiber was then exposed to the beam from a Krypton-Fluoride excimer laser (operating wavelength of $248 \mathrm{~nm}$ ) after passing through a diffractive phase mask and focused along the fiber axis to produce a periodic intensity pattern. The periodic light pattern gets permanently imprinted in the fiber core as a modulation of refractive index with an amplitude of the order of 0.001 after a few minutes of irradiation at 100 pulses per second and a fluence of about $20 \mathrm{~mJ} / \mathrm{pulse} / \mathrm{cm}^{2}$. The grating period of $548.88 \mathrm{~nm}$ and tilt angle of 10 degrees relative to the fiber cross- 
section ensured that the input light guided by the core was coupled to cladding modes with an efficiency of at least $98 \%$ between wavelengths of $1543 \mathrm{~nm}$ and $1552 \mathrm{~nm}$ as shown in the transmission spectrum of Figure 4.1.

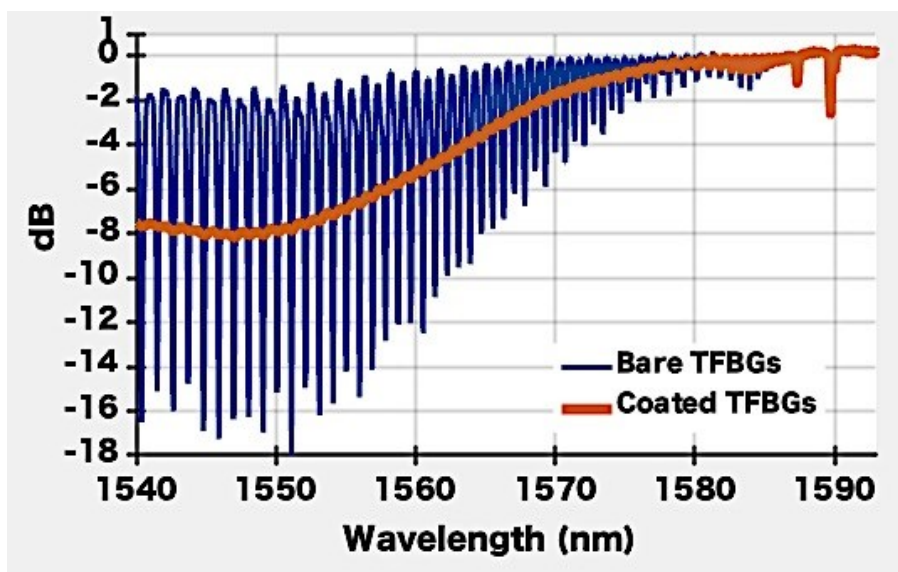

Figure 4.1. Transmission spectrum of the TFBG-based active heater.

\section{1. 2 Heating Setup for the TFBG-based Heater:}

The heating source in the experimental setup consists of a C-band tunable laser and an erbium-doped fiber amplifier (Figure 4.2). TFBG-based heaters couple the amplified light to the coating layer in which it gets absorbed and turned into heat. The changes in temperature are then monitored using $\mathrm{C}+\mathrm{L}$ band broadband source and optical spectrum analyser in reflection through observing the shifts in the TFBG-based heaters Bragg resonance. We use the Lab View to record the temperature increase as a function of the pump power and time. 


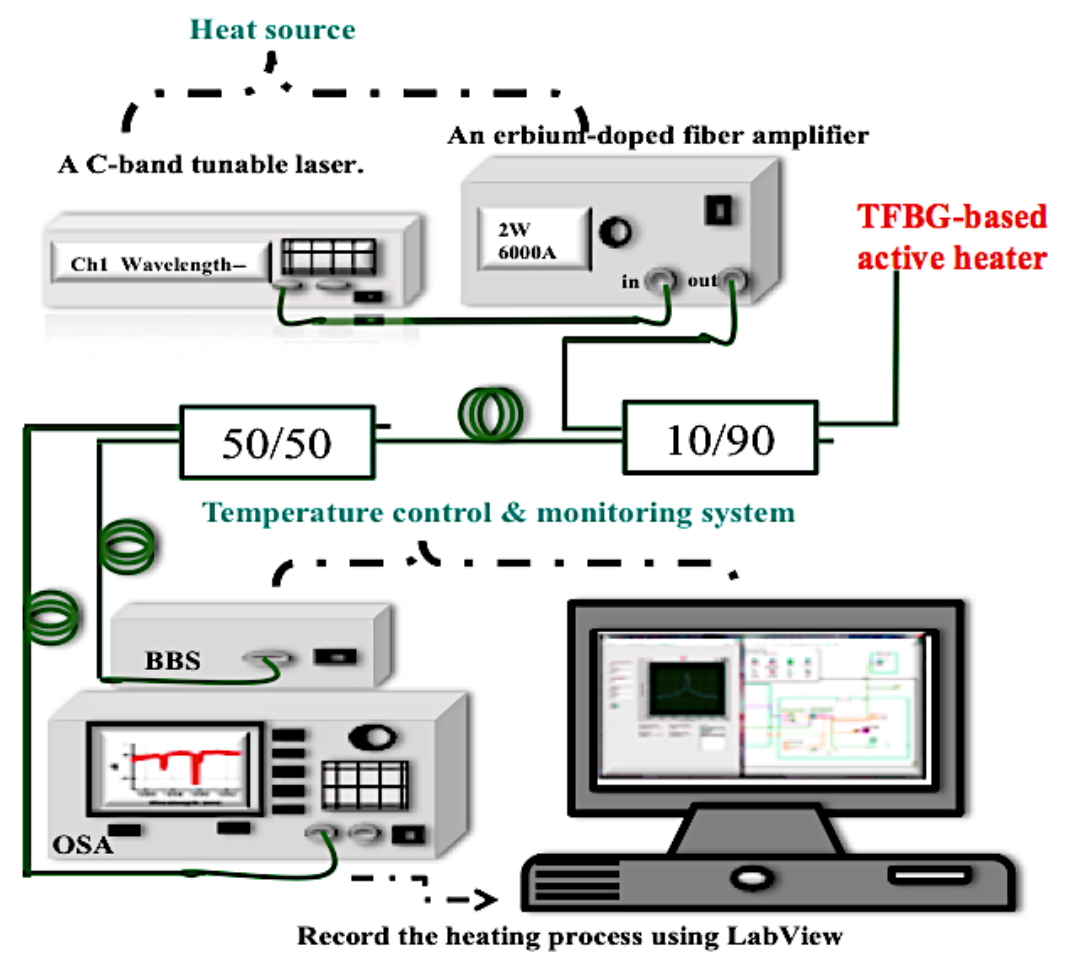

Figure 4.2. Schematic diagram of the heating setup.

\section{1. 3 TFBG-based Heater Preparations:}

After cleaning all of the devices by the piranha solution, TFBGs are either coated directly by a near infrared absorptive material or else packaged in a stainless-steel needle. The preparation process begins with cleaning TFBGs in piranha solution for one hour. The piranha solution is a 3:1 synthesis mixture of sulfuric acid $\left(\mathrm{H}_{2} \mathrm{SO}_{4}\right)$ with hydrogen peroxide $\left(\mathrm{H}_{2} \mathrm{O}_{2}\right)$. We have included this primary step in our work after encountering anomalous results in the heating efficiency of TFBGs devices, even when using the same device to conduct the heating. As an additional challenge, the coating material blows off the fiber surface easily while running the experiment. These two problems had not been encountered when running the experiments at cold and low humidity in the lab. 
Thus, we took the transmission spectrum of a bare TFBG before and after cleaning by the piranha solution (Figure 4.3). The spectra are taken after placing the TFBG in an oven with a temperature setting of $25^{\circ} \mathrm{C}$. We observed shifts to longer wavelengths in the high order modes of the TFBG due to removing the water adsorption on the fiber surface which depends on the relative humidity of the surrounding air. This behaviour is in agreement with the reported results in the literature. Silicon dioxide is an active material in the presence of water molecules in the air [207]. Dioxide-air interference can affect the electrical conductivity of the silicon dioxide, thereby restricting its direct use in some applications including gate field-effect transistor. In our case, this layer may cause part of the large deviation in the heating efficiency, an effect which we had experienced previously between the experiments performed in the summer and in the winter.

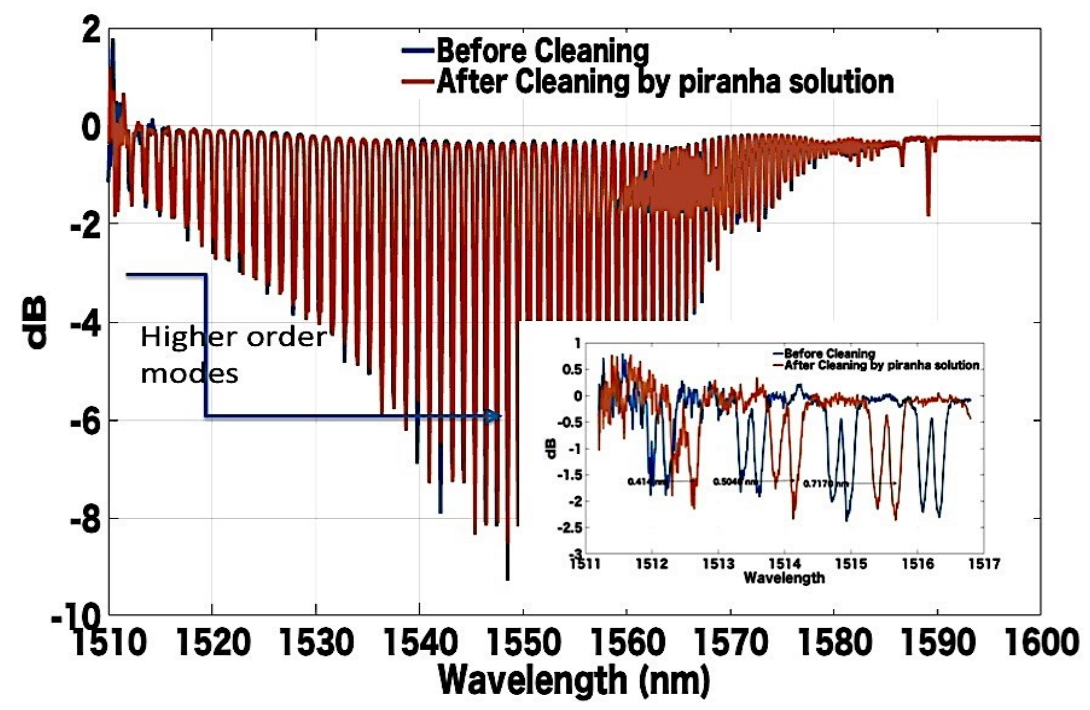

Figure 4.3. Transmission spectrum of TFBG before and after cleaning taken in the oven.

\section{1. 3. a Coating Materials Evaluation for the TFBG-based Heater:}

We have evaluated the heating efficiency of two types of coatings containing NIR absorptive materials, a coating containing NIR reflective material, and two different 
thicknesses of gold films. The dip coating technique is applied while coating the NIR absorptive and refractive materials. The gold films are deposited using the electroless deposition approach.

NIR absorptive materials are oxides of cobalt and aluminum "known as a cobalt blue" and amorphous carbon pigments. These materials are deeply coloured from very dark blue to black and show intense absorption in the NIR region from 1500 to $2100 \mathrm{~nm}$. They are sometimes used as colouring agents. We purchased the cobalt blue and the carbon pigment from M. Graham Watercolors. Both coatings are used as a means to devise the experiment conditions and the device performance as a function of the pumping power and time. As shown in Figure 4.4, the cobalt blue and the carbon coating show an increase in temperature up to 73 and $31^{\circ} \mathrm{C}$ respectively in $45 \mathrm{ml}$ water contained in the test tube and placed in the oven with a temperature setting of $22^{\circ} \mathrm{C}$. Figure 4.5 shows the Bragg resonance shift in correspondence to the coated layer temperature.

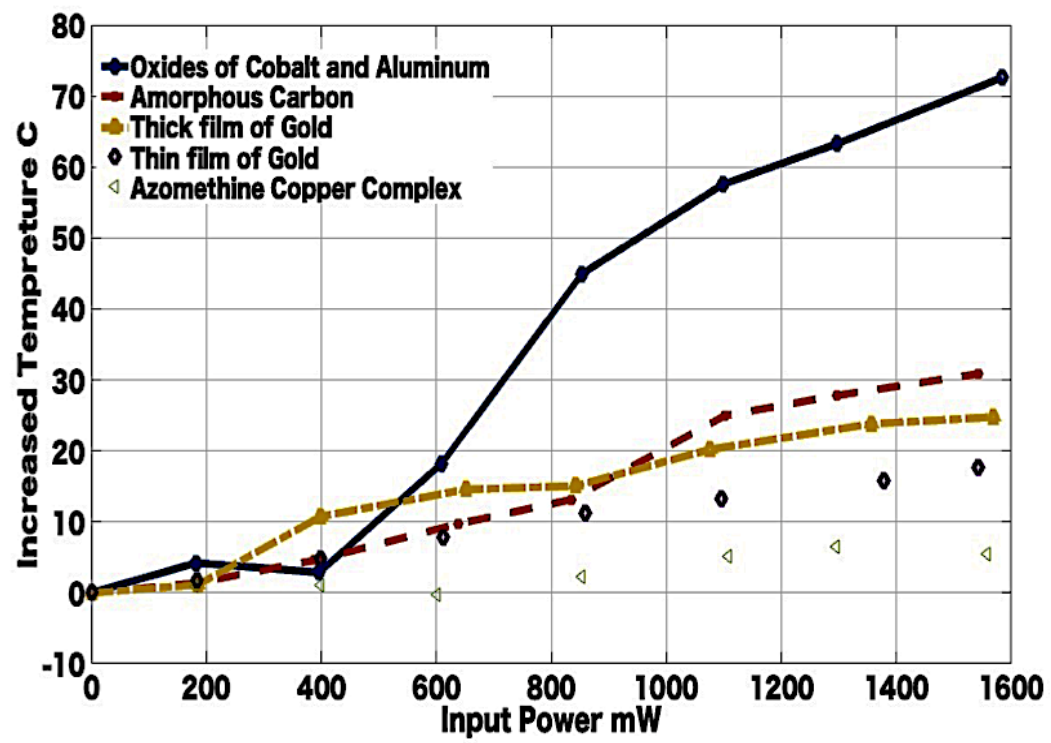

Figure 4.4. Effect of different type of coatings on the heating efficiency of TFBG-based active heaters as a function of the increase in the pump power in increment of $200 \mathrm{~mW}$ per minute. 


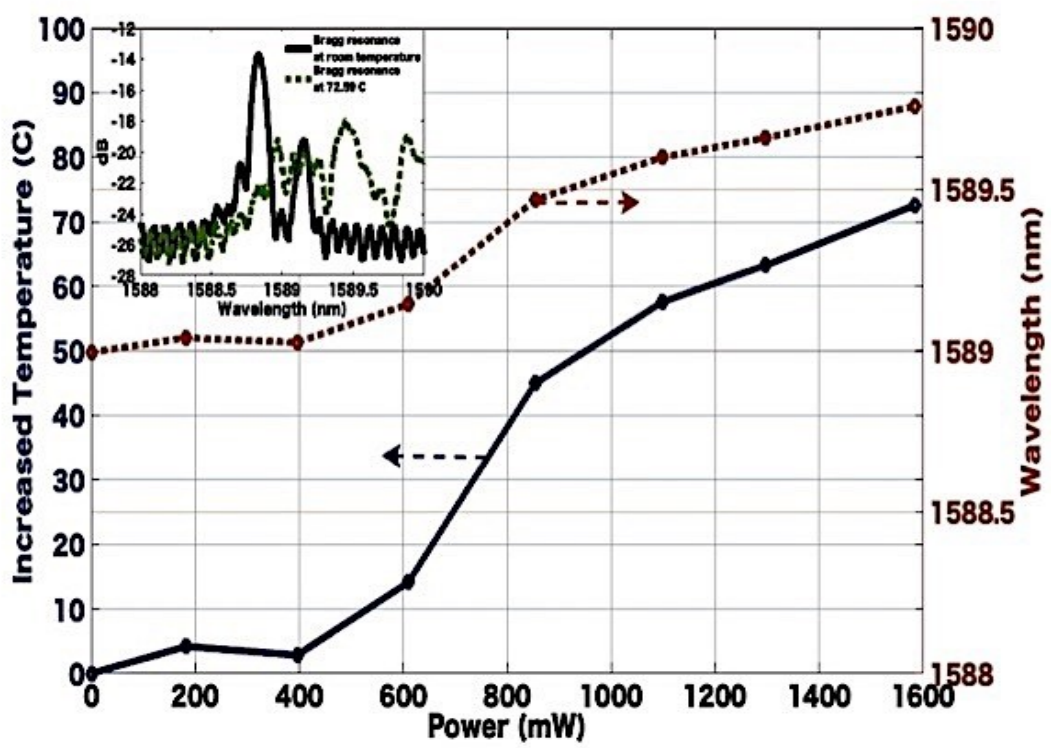

Figure 4.5. Bragg resonance shift in correspondence to the coated layer temperature; reflection spectrum of the cobalt blue coated TFBG-based active heater.

NIR reflective materials are azomethine copper complex pigment and gold films. These reflective materials manifest different levels of heating efficiency (Figure 4.4). Negligible heat was observed from the azomethine copper complex pigment. In comparison, heating achieved through coated TFBGs by gold films are in order of 18 and $24{ }^{\circ} \mathrm{C}$ from the thin and thick films respectively. The utilization of gold film coating as a heater was excluded due to poor adhesion to the fiber surface and low heating efficiency compared to that obtained from the NIR absorptive pigments.

\section{1. 3. b Packaging the TFBG-based Heater:}

The task of packaging the TFBGs was challenging because the needle has to be filled by a conductive material while the fiber is inside the needle. The needle's outer and inner diameters are approximately 508 micrometers and 260 micrometers respectively. Thus, the large force which must be applied when pushing the conductive material to the small diameter needle increases the risk of breaking the gratings. This conductive material is 
needed to remove the air gap, which acts as an isolated layer, between the TFBG and the needle wall.

\section{1. 4 Heated Target Materials:}

\section{1. 4. a Cell Lines and Cell Cultures:}

HEK 293, MCF-10A, and MCF7 cells were purchased from American Type Culture Collection (ATCC). HEK 293 embryonic kidney cells were cultured in $90 \%$ DMEM and $10 \%$ fetal bovine serum (FBS). MCF-10A epithelial breast cell line was cultured in Mammary Epithelial Cell Growth Medium (MEGM) Bulletkit. MCF7 breast cancer cell line was cultured in $90 \%$ DMEM, $10 \% \mathrm{FBS}$, and $10 \mu \mathrm{g} / \mathrm{mL}$ insulin. All three cell lines were maintained at $37{ }^{\circ} \mathrm{C}$ with a $5 \% \mathrm{CO}_{2}$ humidified environment.

To prepare the cells for the thermal therapy, the active heaters were fixed in $6 \mathrm{~cm}$ plates. All three cells were then subcultured on top of the active heaters (Figure 4. 6). After the treatments, the cells' media were changed and the plates were incubated for 3 to 4 hours at $37^{\circ} \mathrm{C}$ and $5 \% \mathrm{CO}_{2}$ humidity. Trypan blue (TB) dye was then used to stain only the nonviable cells. The effects of hyperthermia on the cells were captured by taking microscopic images. All experimental groups were compared to their own cell line control of cells not treated with heat. We then used ImageJ toolbox to determine the length of the killing zones associated with a range of hyperthermic temperatures. 


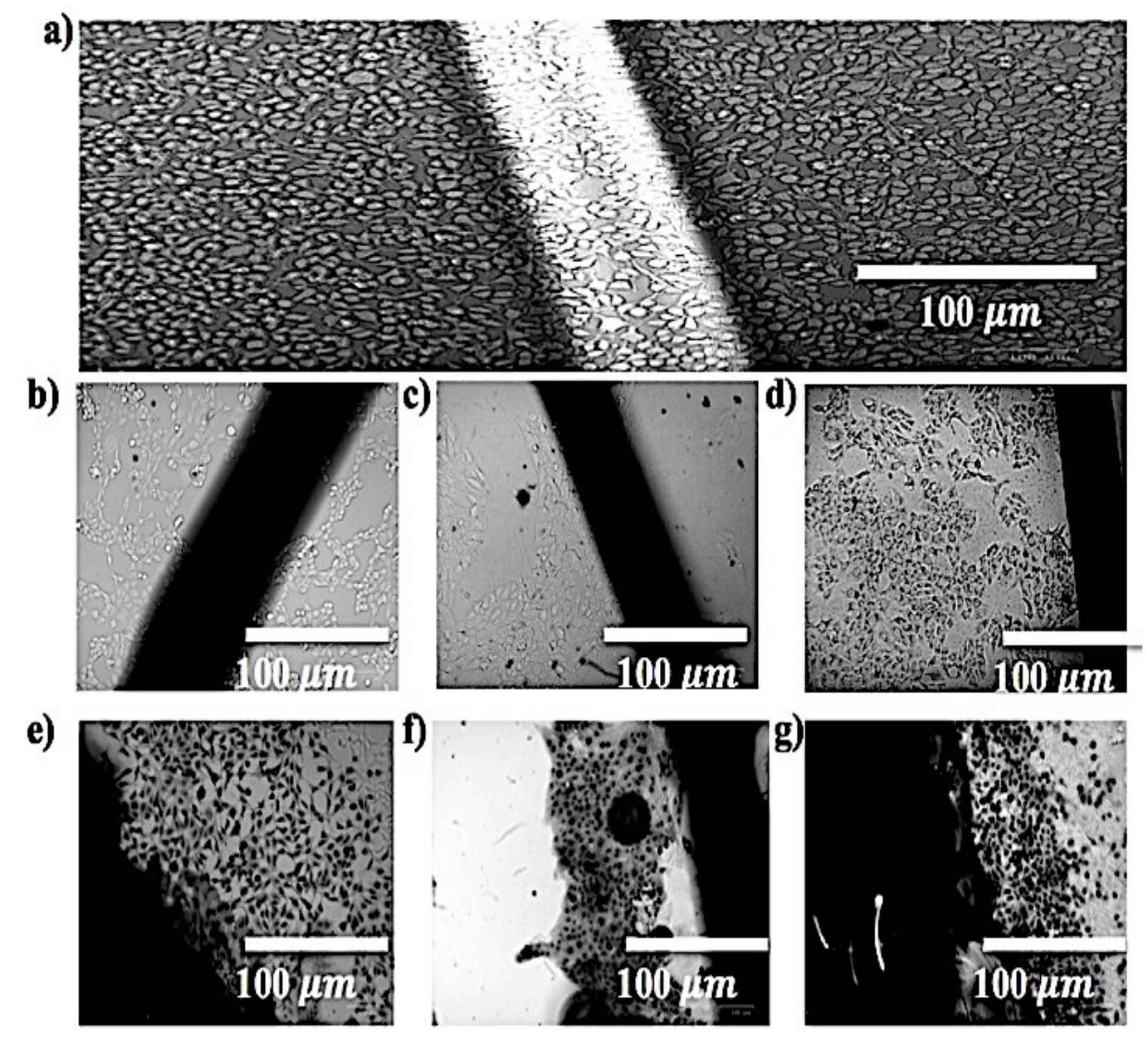

Figure 4.6. Cells grown in $6 \mathrm{~cm}$ plates on top of the active heaters; (a \& b) HEK 293 cells on top of a bare active heater: (a) for just visualisation: we can not see the cells grown on top of the active heater due to the black color of the coating layer; (c) MCF-10A and (d) MCF7 cells grown on top of the coated active heater. (e) HEK 293 (f) MCF-10A and (g) MCF7 cells viability after exposing to heat with maximum temperatures of 54 ${ }^{\circ} \mathrm{C}$ for 5 minutes; after the treatment we used TB to stain only the dead cells.

Cells counting were used to conduct further assessment of the MCF-10A and MCF7

cells' sensitivity to hyperthermia. MCF-10A and MCF7 cells were cultured on top of the

active heater in $6 \mathrm{~cm}$ plates. After undergoing hyperthermia, the plates were incubated for

3 to 4 hours at $37{ }^{\circ} \mathrm{C}$ and $5 \% \mathrm{CO}_{2}$ humidity. Cells were then gently washed twice with 1 
$\times$ phosphate buffer solution (PBS) and trypsinised. When the cells started to become rounded and detached from the plates, media was added to neutralize the trypsin. We counted the surviving cells manually under the microscope using hemocytometer after the cells had been stained by TB to differentiate between the dead and live cells. Cell viabilities were normalized to cells grown under the same condition without exposure to hyperthermia.

\section{1. 4. b Tissue-Like Materials:}

The ability of the TFBG-based active heater to cause damage in cancer tissues and provide real-time feedback about the degrees of temperature increase is investigated experimentally in phantoms. Two types of materials - egg white and gelatine - used to mimic the heat conductivity of human tissues are chosen from the literature. These two phantoms are favoured over other types of false tissues due to their transparency for lesion visualization, their wide availability, and their low cost.

\section{1. 4. b. i Gelatine-based Phantom:}

Gelatine-based phantom is prepared from $30 \mathrm{ml}$ water and jello powder (Figure 4.7) The jelly was frozen, so it was left at room temperature for 30 minutes prior to running the experiment to obtain a soft high-water concentration phantom. 


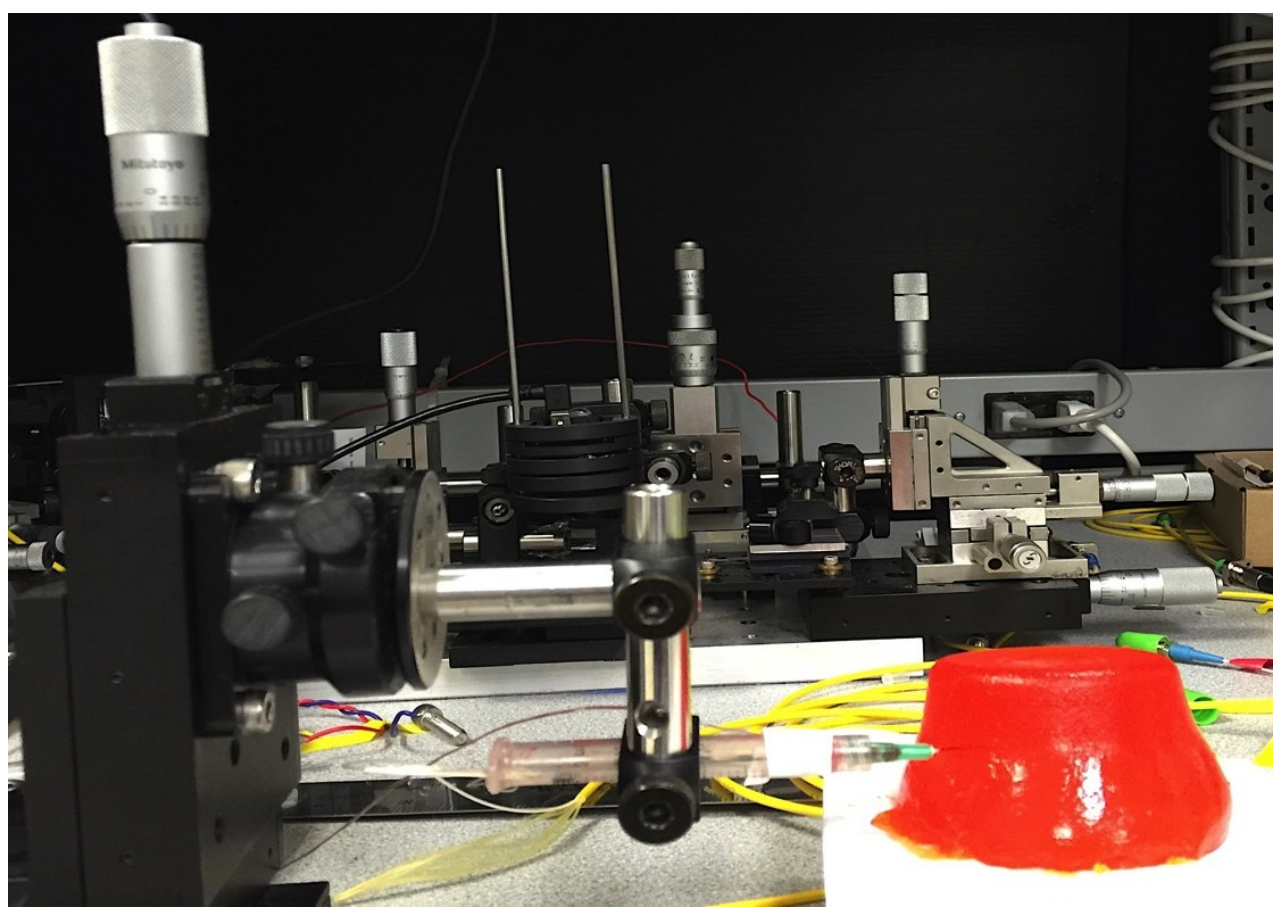

Figure 4.7. TFBG-based active heater enclosed in the needle inside the jelly.

\section{1. 4. b. ii Egg White-based Phantom:}

We used the egg white-based phantom to emulate the performance of the active heater in quasi- conductor media. The approximate thermal conductivity and heat capacity of some human tissues with a high concentration of water, such as the liver, can be mimicked by using fresh egg white. Similar to human blood which fills tissues, water is the major constituent (of the order of $90 \%$ ) of the hen egg white dissolved into proteins. Figure 4.8 shows the calculated effect of heat-induced hyperthermia in the hen egg white, porcine liver, and human liver under similar conditions to the experimental setup with heating for 10 -minute at $1.8 \mathrm{~W}$ without a source of heat loss which is the blood perfusion in living tissue. 


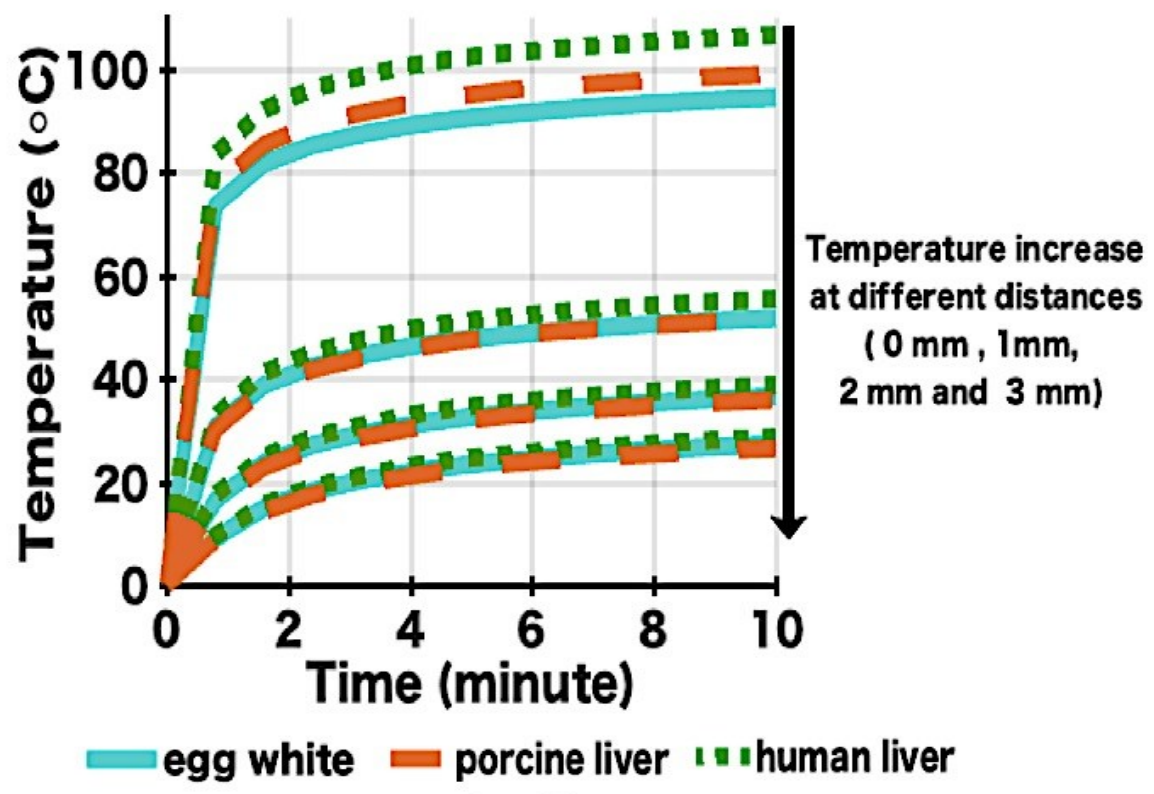

Figure 4.8. The calculated effect of heating at pump power of $1.8 \mathrm{~W}$ for 10 minutes in the hen egg white, human and porcine liver without considering the blood perfusion effect.

Characterization of thermochromic ink colour change: The thermochromic ink permanently and gradually changes colour from white to magenta when exposed to heat as the temperature increases from room temperature until the colour saturates at $70{ }^{\circ} \mathrm{C}$. We calibrate the colour change of the ink by using capped vials containing DI water and the thermochromic ink at a ratio of 3:1 submerged in a water bath. This ratio was selected experimentally so that the ink concentration was modified while still producing good colour intensity. We measured the temperature of the solution every fifteen minutes using a thermocouple. When the desired temperature was reached, the capped vial was taken out and left to cool to room temperature. Figure 4.9 indicates the permanent colour change of the thermochromic ink in response to the change in temperature from $23^{\circ} \mathrm{C}$ to $70^{\circ} \mathrm{C}$. 


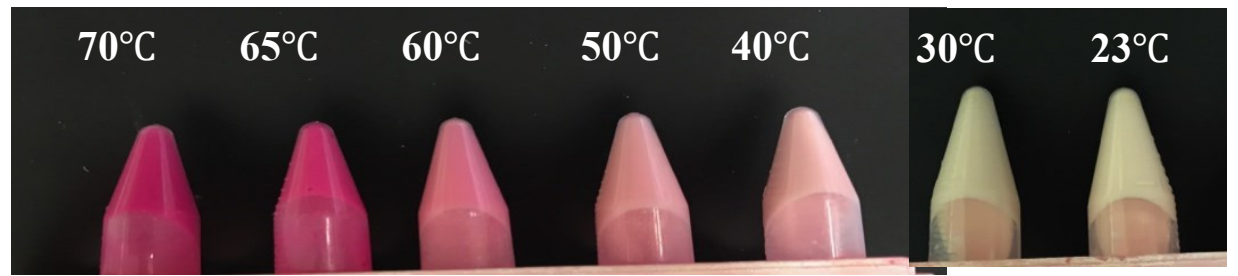

Figure 4.9. Permanent colour changes of the thermochromic ink vs temperatures.

The phantom material was then prepared from the egg white and thermochromic ink using the same ratio of 3:1. We used these vials for qualitative assessment of temperature gradients of the phantom material after heating by the active heater.

\section{1. 5 Ex Vivo Porcine Liver:}

For the ex vivo studies, the active heaters were sandwiched between porcine liver tissues which were purchased from local store (Figure 4.10). The thermal therapy was performed at pump power of $1.8 \mathrm{~W}$ for 5 - and 10-minutes at room temperature. We choose the

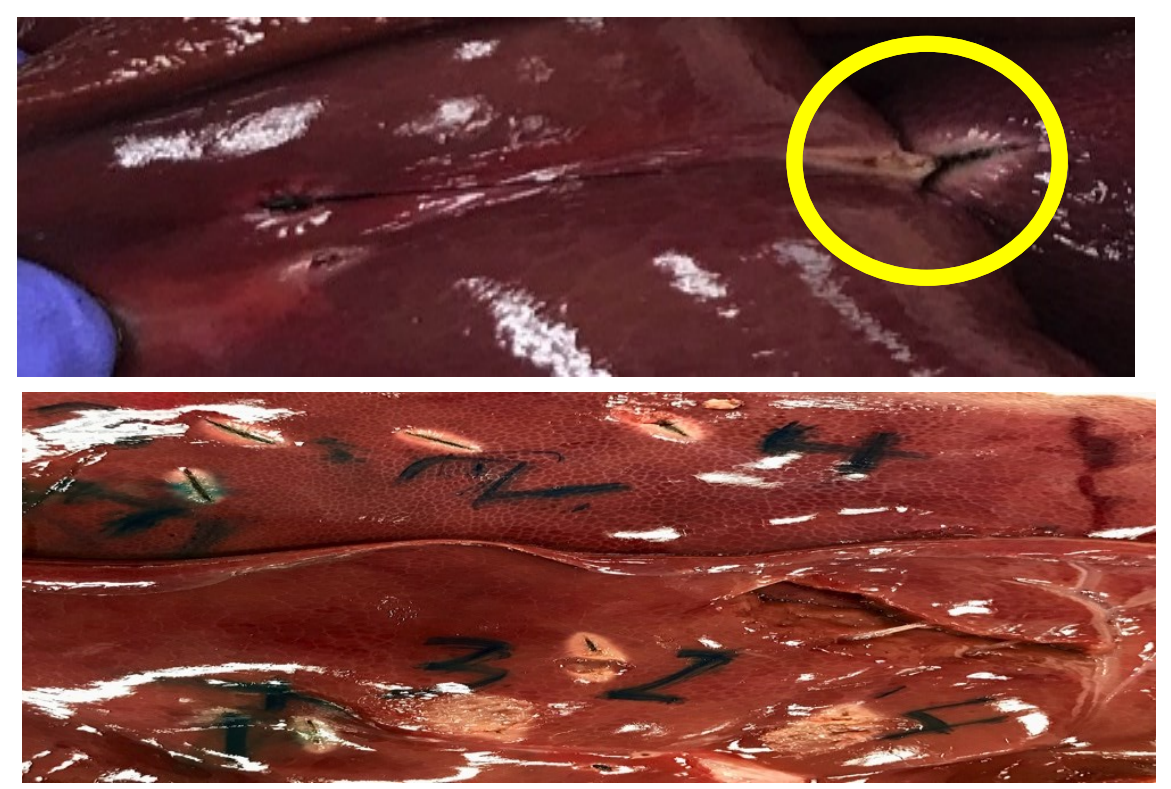

Figure 4.10 Hyperthermia under the same pump power in order of $1.8 \mathrm{~W}$ for 5 - and 10- minutes; top (upper panel) the active heaters were sandwiched between the liver tissues; side (lower panel) lesions number 1 and 2 result from continuous heating for 5 minutes, lesions number 3 and 4 result from continuous heating for 10 minutes.

porcine liver when performing the ex vivo studies because this mammalian species is

known to have metabolic and physiological similarities to humans. Figure 4.8 shows the 
similarities of the heat-induced lethal volume in the porcine liver and human liver.

\section{2 Annealing System to Evaluate the Gratings Thermal Stability:}

\section{2. 1 Ultrafast FBGs Fabrication Conditions:}

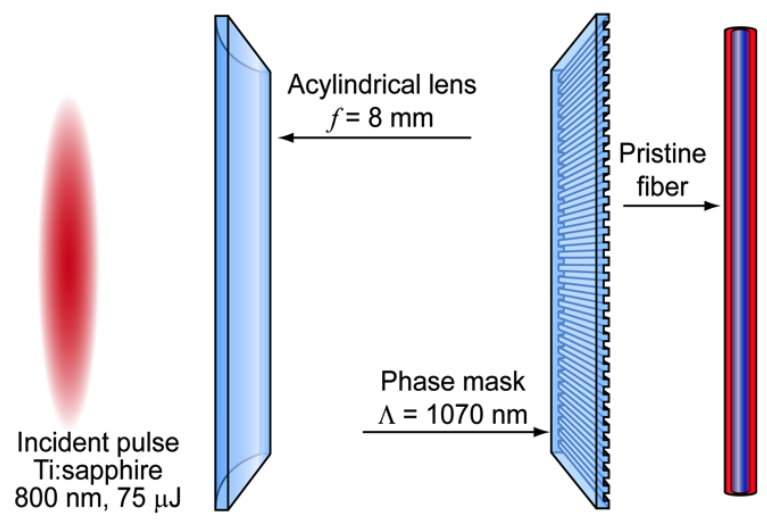

Figure 4.11. Exposure conditions used to write the FBGs through the polyimide coating.

Figure 4.11 shows the schematic of the FBG writing setup for both the $1^{\text {st }}$ and $3^{\text {rd }}$ order devices. A Ti:sapphire regenerative amplifier system (Coherent, model Legend-HE) with a repetition rate of $1 \mathrm{kHz}$ and a central wavelength of $806 \mathrm{~nm}$ was utilized to write the gratings in SMF-28 fiber [212]. Prior to writing, the fiber was loaded with hydrogen in a pressurized chamber at $2000 \mathrm{psi}$ and room temperature for 14 days. The pulse energy was set to $90 \mu \mathrm{J}$ and $120 \mu \mathrm{J}$ for the first and third order devices respectively. These writing conditions, even though the energy was slightly higher for the $3^{\text {rd }}$ order devices, produce intensities well below the type II damage threshold $[213,214]$. The output laser beam having a diameter of $8.5 \mathrm{~mm}$ at 1/e 2 intensity was directly focused using a $8 \mathrm{~mm}$ focal length acylindrical lens (Thorlabs AYL108-B) through the phase-mask having a pitch of respectively 1.07 and $3.21 \mu \mathrm{m}$ for the first and third order gratings. The $1^{\text {st }}$ order-devices could easily be written through the fiber jacket as expected [212, 322], while the $3^{\text {rd }}$ order-devices required the jacket to be stripped on account of damage to the acrylate coating. The fiber was placed at a distance of approximately $125 \mu \mathrm{m}$ away from the 
phase-mask. The fiber sample was scanned vertically by $\pm 10 \mu \mathrm{m}$ with a piezoelectric stage at a frequency of $1 \mathrm{~Hz}$ so that the beam subtended the fiber core. The first order grating will grow monotonically until it reaches its saturation point. While the $3^{\text {rd }}$ order device will grow initially until it is arrested, begins to erase, and then grows again before reaching its saturation point (which is not necessarily the same as that of the first order devices) (Figure 4.12).

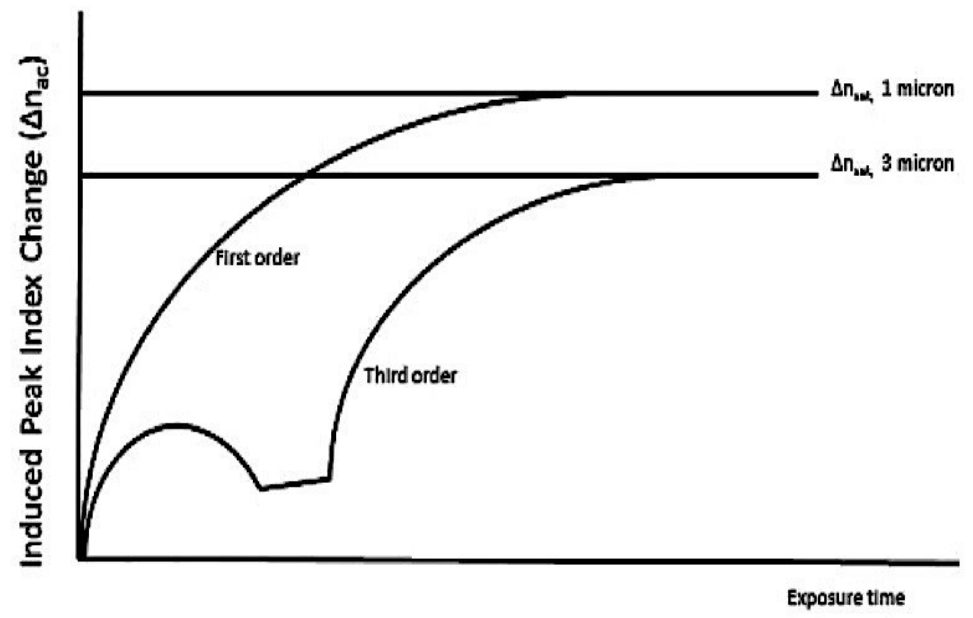

Figure 4.12. The growth behavior of first and third-order gratings.

\section{2. 2 Ultrafast FBGs Annealing Setup:}

The first and third order gratings were heated isothermally to $950{ }^{\circ} \mathrm{C}$ in increments of 10 ${ }^{\circ} \mathrm{C}$ per minute. After this, gratings were kept at $950{ }^{\circ} \mathrm{C}$ and $1000{ }^{\circ} \mathrm{C}$ for 12 hours before cooling down to room temperature. The annealing was performed in ambient atmosphere. The gratings were situated loosely in a middle of the $60 \mathrm{~cm}$ Carbolite tube furnace as shown in Figure 4.13. 


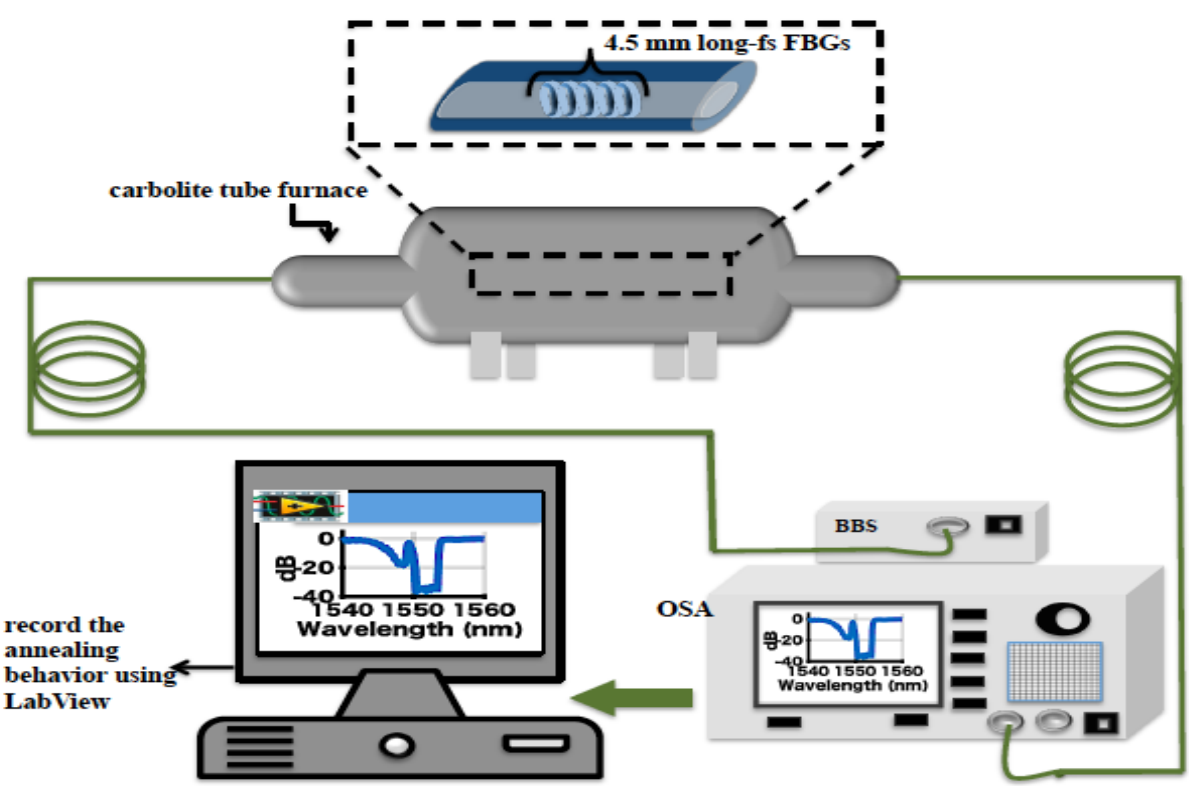

Figure 4.13. Schematic diagram of the annealing setup.

The effect of the annealing process on the transmission spectra of both gratings was monitored by a broadband light source (AFC/BBS 1550 A-TS) together with an optical spectrum analyzer (Agilent 86142B) adjusted at a resolution bandwidth of $0.06 \mathrm{~nm}$.

During the experiment, the transmission loss at the Bragg resonance of the gratings in the furnace was recorded through the GPIB interface between the Agilent 86142B and a LabView computer package at three minutes intervals. 


\section{Chapter 5: Results and Discussions}

We start this chapter by showing the effect of the optical properties of the TFBG-based active heater on heating efficiency. These primary experiments are performed in water. We then show the in vitro hyperthermia-induced cell-killing results followed by the heating results at the tissue level. Finally, we finish this chapter by describing the packaged TFBG-based active heater and its thermal stability.

\section{1 Optical Properties of the TFBG-based Heater:}

We performed primary tests on the TFBG-based heater in $45 \mathrm{ml}$ of water contained in the test tube and placed on the lab bench to find the optimal heating setup, the TFBGs' properties, and the effect of pump power modes on the heating efficiency. Figure 5.1 compares the effect of two pump modes, namely sweep mode over range of wavelengths and single wavelength mode, on the heating efficiency of the active heater. Heat

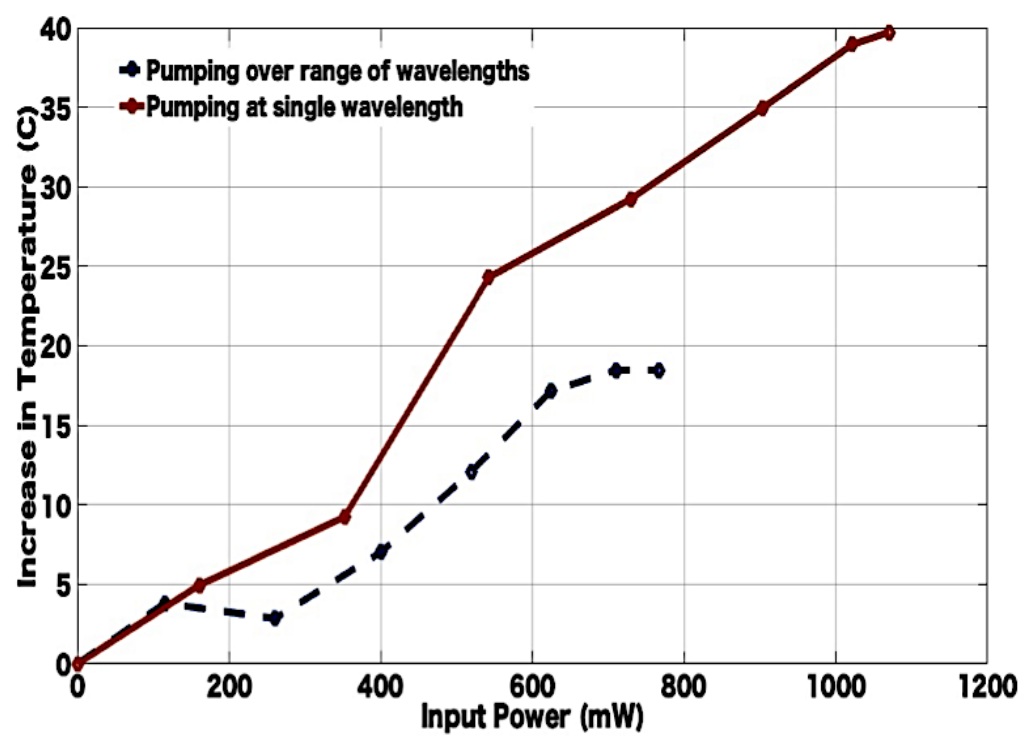

Figure 5.1. A comparison between pumping at single wavelength and over a range of wavelengths on the heating efficiency. 
generates from the heater as the pump power passes through the fiber, which causes a shift in the TFBGs' spectra, core mode, and cladding modes, to longer wavelengths. Pumping at a single wavelength of $1547 \mathrm{~nm}$ provides better heating efficiency than pumping over the range of wavelengths of 1547 to $1552 \mathrm{~nm}$. Even though we had used the same setting in the amplifier, the maximum pump power obtained from pumping over the range of wavelengths was on order of $766 \mathrm{~mW}$. The laser power is divided into this range of wavelengths and the slow sweeping cycle of the NIR laser, the total input power becomes low.
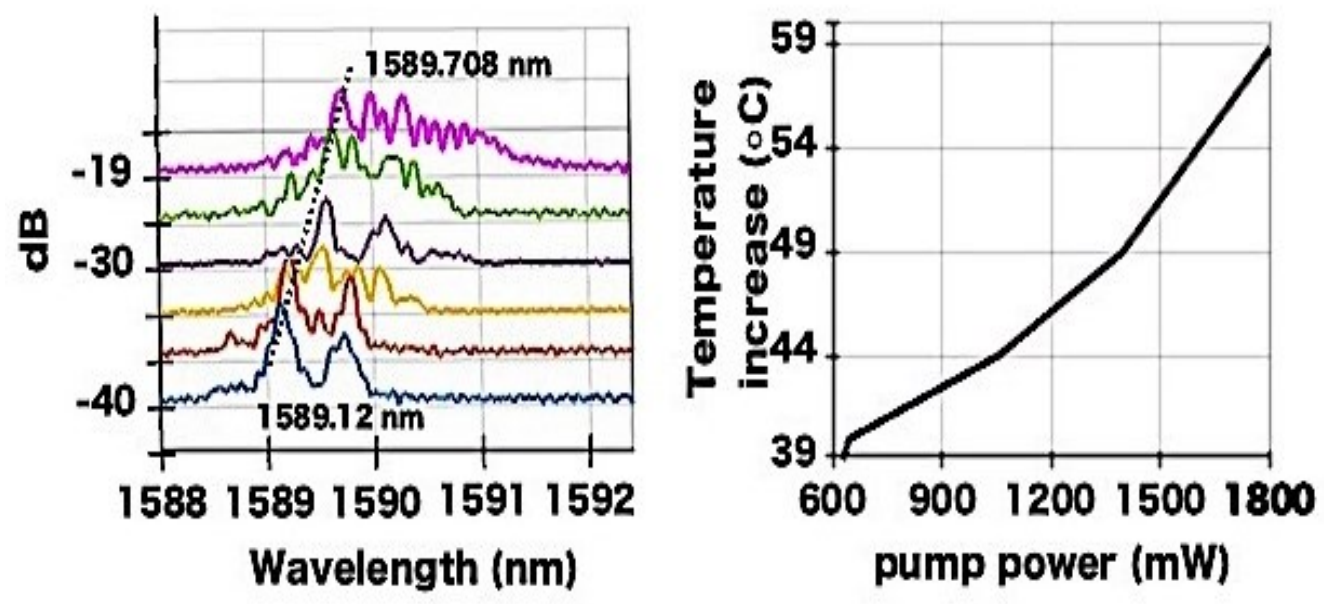

Figure 5.2. Bragg wavelength shift as a function of the pump power increase (left), the corresponding temperature change (right).

Figure 5.2. shows that heat produced by the active heater is proportional to the pump power. A pump power of $1.4 \mathrm{~W}$ results in a temperature increases of $38{ }^{\circ} \mathrm{C}$ in water. The strength of the index modulation also influences the coupling efficiency of the cladding mode resonance, thereby, the amount of power used for heating. TFBGs with strong index modulation couple more power to the coating layer compared to the gratings with moderate and weak index modulation. After coating the active heaters, cladding mode 
resonances with a reflectivity of $75 \%, 96 \%$, and $98 \%$ turned into radiation modes with a reflectivity of $60 \%, 75 \%$, and $84 \%$ respectively (Figure $5.3 \mathrm{a}$ ). Thus, $60 \%, 75 \%$, and $84 \%$ of the pump power is used to generate heat in water. Temperature increases of $20.4,37$, and $48.5^{\circ} \mathrm{C}$ when pumping at $1.4 \mathrm{~W}$ are obtained from these heaters as their index modulation strength increases (Figure 5.3b).

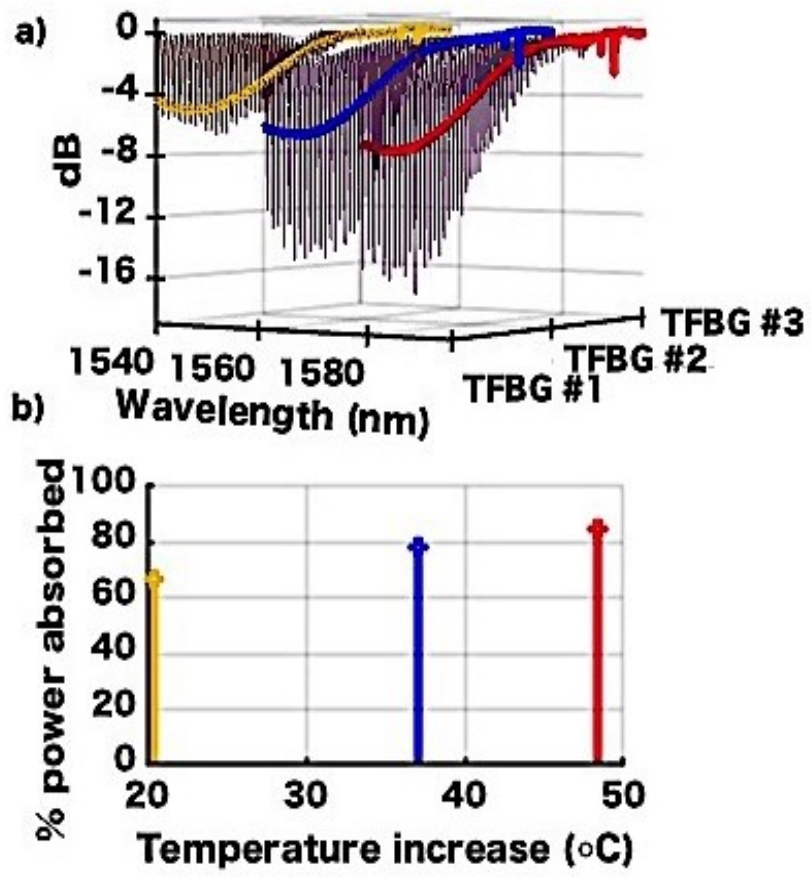

Figure 5.3. (a) Transmission spectra of TFBGs with different strengths $(6,14, \& 16.7 \mathrm{~dB})$ with their transmission spectra after applying the absorptive coating layer; (b) temperature increase at a fixed power of $1.4 \mathrm{~W}$ as a function of increasing grating strength measured in water.

Using the same conditions to write these active heaters produces the same heating profile, as shown in Figure 5.4. An approximate of $60 \%$ of $1.44 \mathrm{~W}$ pump power is converted from light to heat by the coating layer on the active heaters which have similar strength. Similar amount of heat is generated from these active heaters. Chart $5.4 \mathrm{~b}$ indicates total temperature difference of $\pm 4{ }^{\circ} \mathrm{C}$ and a minimum temperature of $22{ }^{\circ} \mathrm{C}$ is obtained from these active heaters under the same pump power. FBGs thermometers are also used here to confirm the temperature reading from the active heaters. 
The degree of the tilt angles in the TFBGs influences the bandwidth of the cladding mode resonances. The 10 degrees tilt angle of our active heater was selected to allow pumping wavelengths away from the Bragg resonance. Also, the length of the device can be changed according to the required size of treated volume.

a)
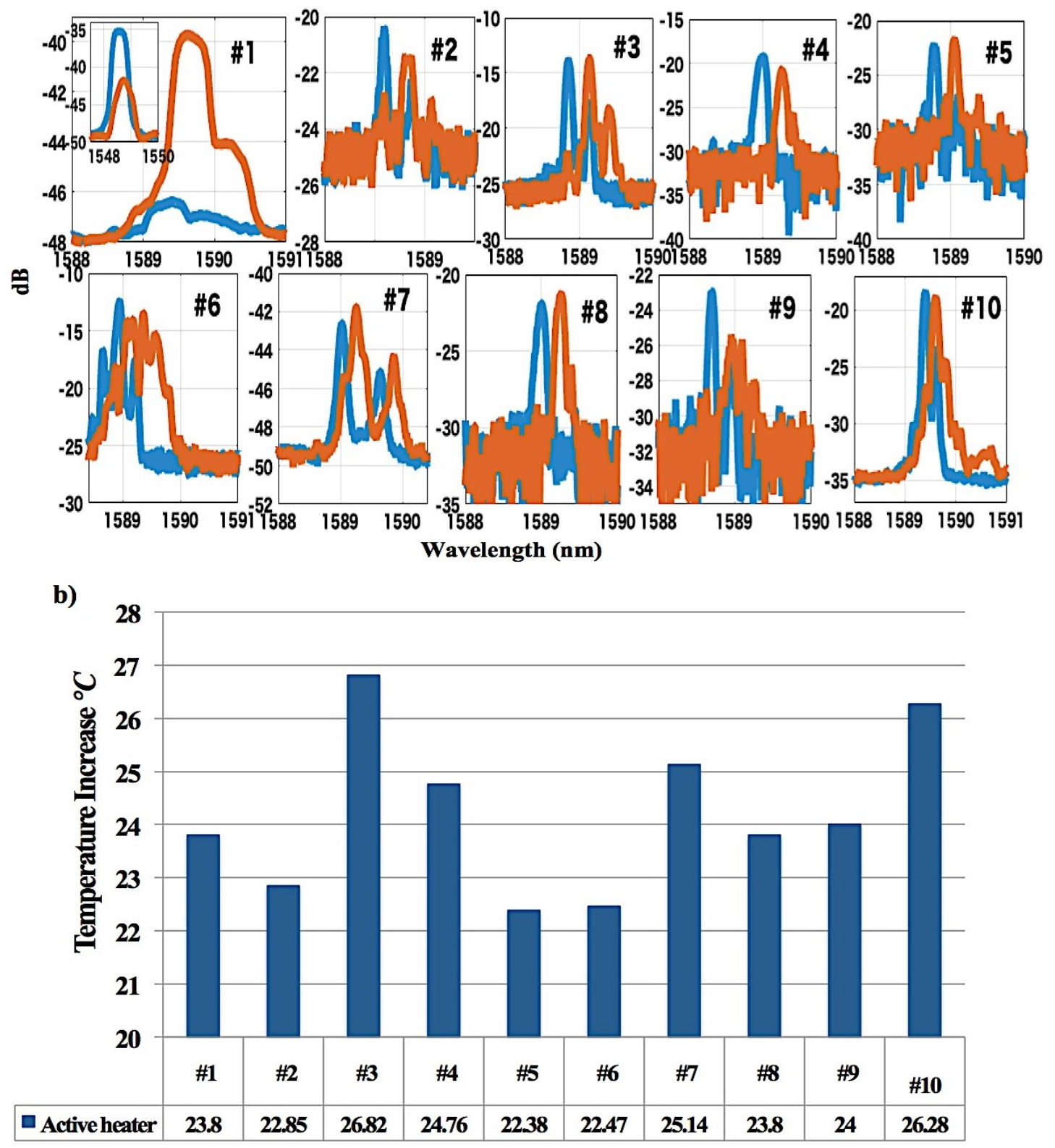

Figure 5.4. Active heater written under the same conditions: (a) reflection spectra of 10 active heaters; (b) the resulting temperature increase 


\section{2 In Vitro Hyperthermia of HEK293, MCF-10A, and MCF7 Cell Lines:}

In this study we focused on the direct effect of thermal therapy on cells. Cell killing is the direct effect of exposing the cells to therapeutic temperatures for different lengths of time. In thermal dosimetry, temperature and time affect the cells undergoing hyperthermia. The complete effect of exposing cells to hyperthermia is usually determined by studying the colony formation and growth rate of the cells such as clonogenics assay. At the cellular level, direct cell-killing is inevitable upon exposure to temperatures above $50{ }^{\circ} \mathrm{C}$ caused by effecting and damaging proteins responsible for DNA replication and DNA repair, thereby DNA damage [215-217]. However, hyperthermia-induced cell-killing also occurs at low therapeutic temperatures ranging from 40 to $47^{\circ} \mathrm{C}$, fever-range hyperthermia, and traditional hyperthermia regime respectively. The amount of damage depends on the cell line and on the combination between time and temperature "thermal dose". The type of source that is used to induce hyperthermia is a further factor that highly influences the amount of damage at those low therapeutic temperatures. Fast and localized temperature increases induce cell killing more than bulk heating, for example, in a water bath. Generally, the fever-range hyperthermic temperatures are toxic to the majority of cell lines by altering their cellular distribution, inhibiting their activates, or developing heat resistance [215-218].

All cell lines were grown on top of the TFBGs and left to reach 70-80 \% confluency before undergoing hyperthermia as described in the Chapter 4. Inducement of heat within the cells by the active heater increases the temperatures of the area around the fiber to a maximum of $54^{\circ} \mathrm{C}$. 
For the HEK293 cells, figure 5.5. shows the ability to produce controlled heat and hence selective and directed HEK293 cell damage. In real time, we control the heat supply to the target volume by monitoring the Bragg resonance shift during treatment for 5 minutes. Therefore, uniform heat is emitted from the heater causing a steady temperature increase during the treatment. This leads to sharp boundary between the stained (dead) cells and the clear cells (living) indicates that the lethal heat zone was confined to a distance of about $200 \mu \mathrm{m}$ on either side of the TFBG heater, as desired in localized hyperthermia applications.

a)

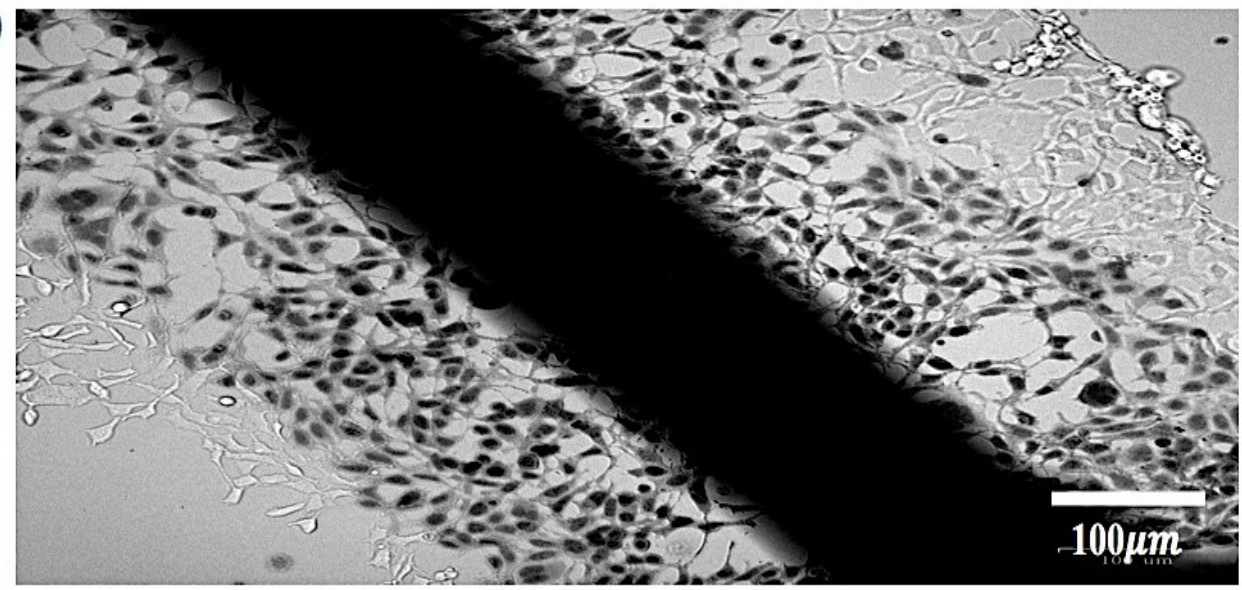

b)

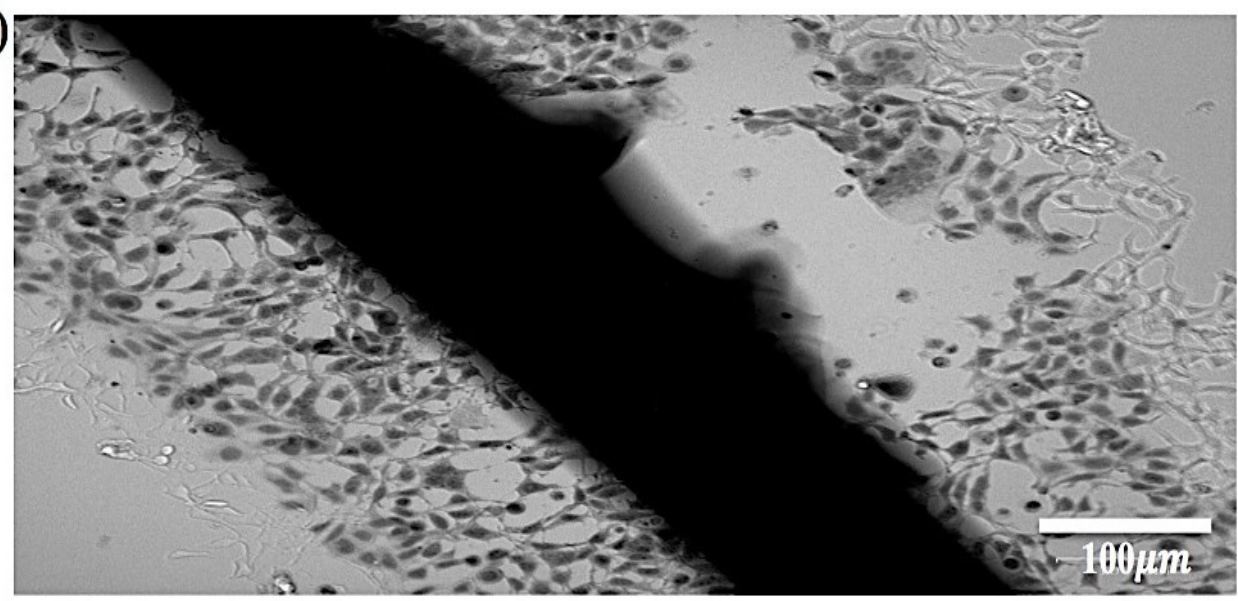

Figure 5.5. (a \& b) HEK 293 cells viability after exposing to heat with temperatures of 53 for 5-minute. After the treatment we used TB to stain only the dead cells. 
In a conduction heat transfer problem, the value of the temperature increase at any locations can be obtained by measuring the rate of heat transfer. Considering a constant change in temperature within a specific distance, from the active heater surface to the FBG's probe located at a distance of $1 \mathrm{~mm}$, we calculate the heat transfer rate in a cell media from the temperature reading at these two points: at the active heater surface and at the FBG's probe. Heat produced by the active heater drops to $23.5^{\circ} \mathrm{C}$ at $1 \mathrm{~mm}$ from the temperature at the active heater's surface $37.71{ }^{\circ} \mathrm{C}$ with heat dissipation rate of $37.5 \%$. Thus, we used the temperature obtained from the active heater with the heat reduction rate to estimate the temperature increase at any points within a distance (a diameter) of 2 $\mathrm{mm}$ around the active heater. Tables in Figures 5.6 5.7, and 5.8 show the minimum toxic temperatures at the points separating the viable cells and the stained dead cells obtained from reduction rate and the reading temperature at the active heater. The locations of these points are determined by using ImageJ toolbox. 
a)

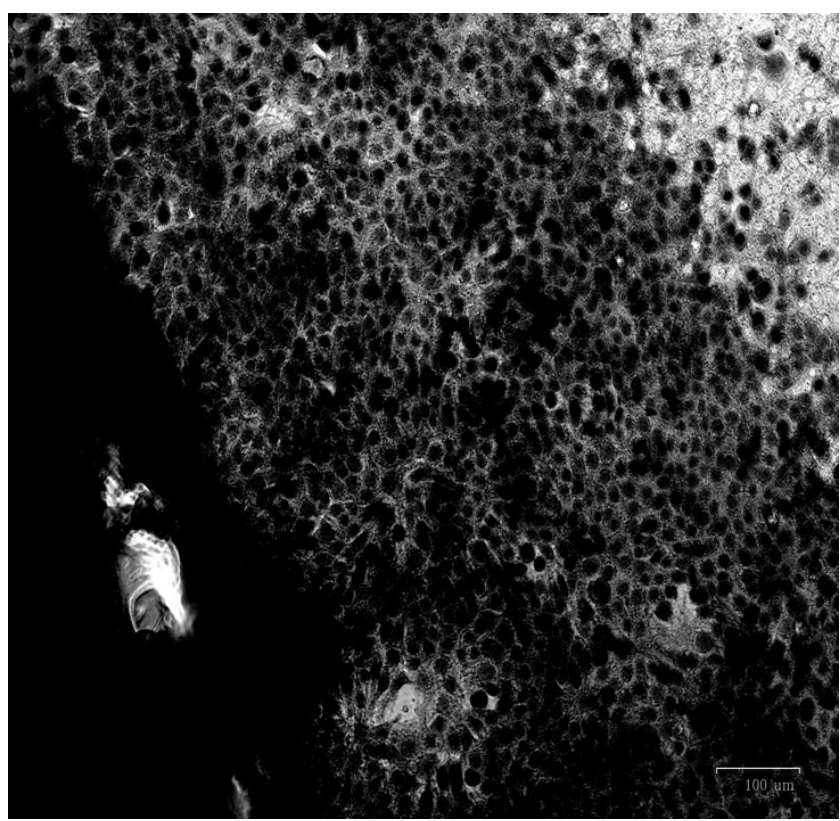

$1.8 \mathrm{~W}$

15 minutes

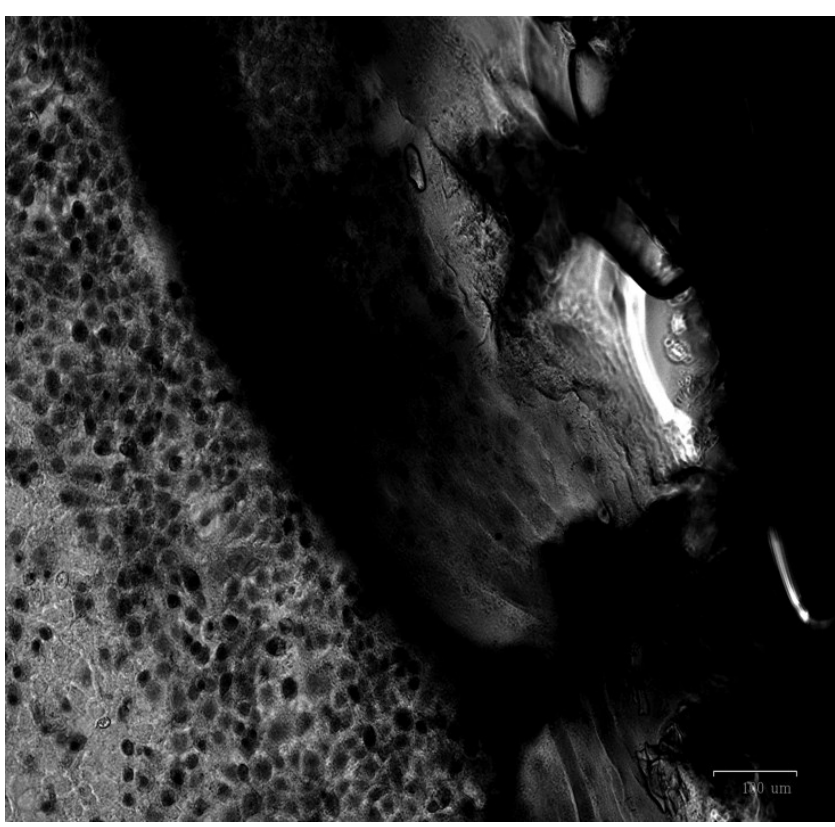

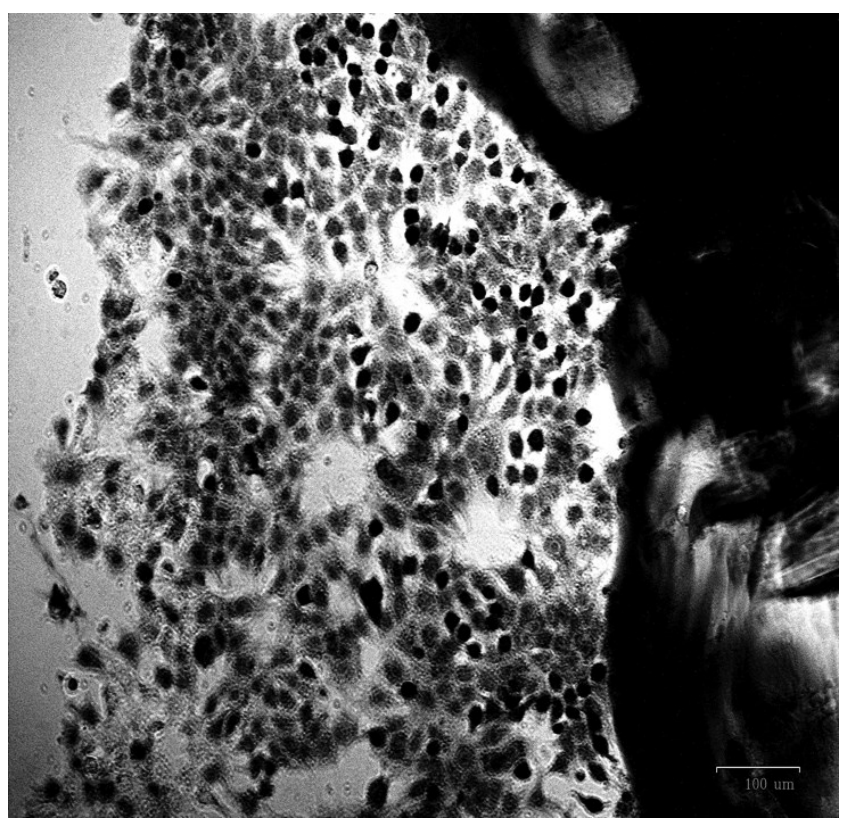

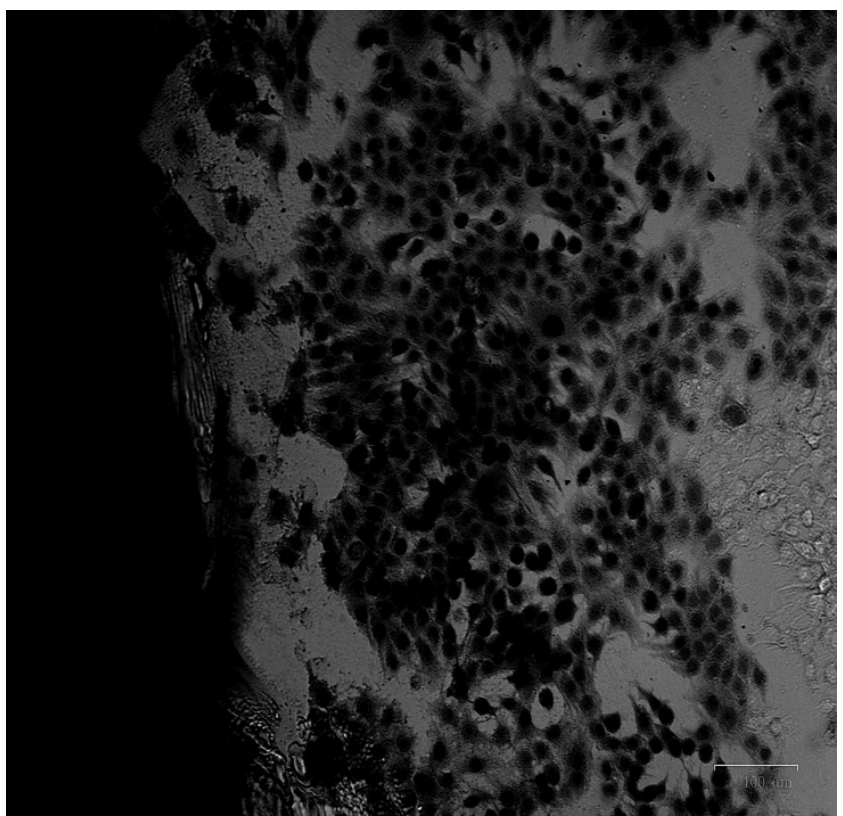


$1.62 \mathrm{~W}$

15 minutes

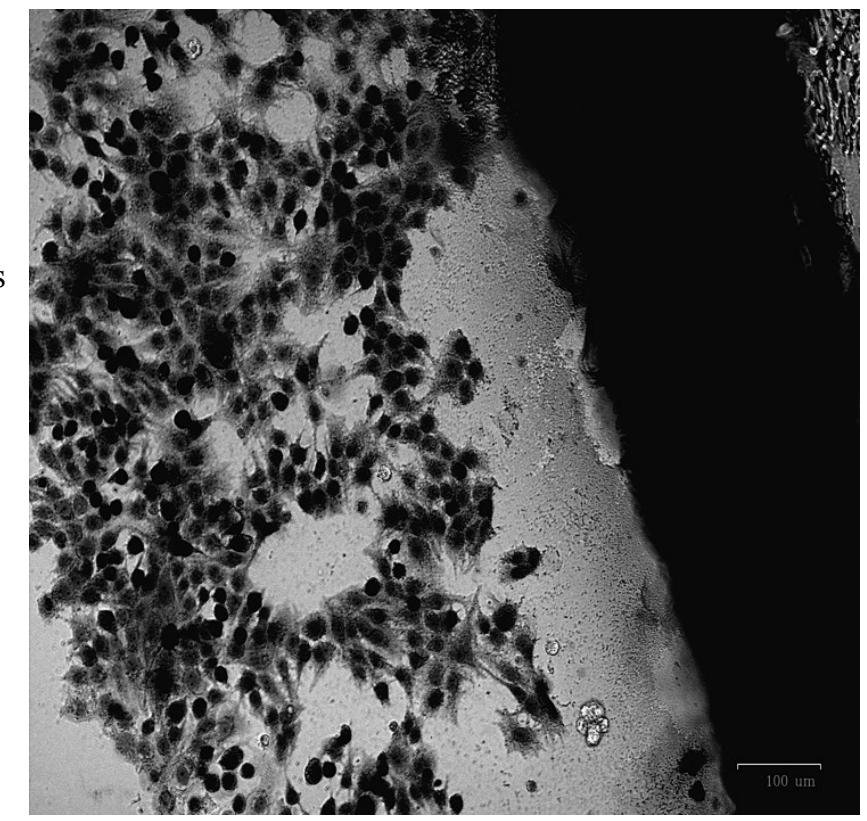

d)

$1.62 \mathrm{~W}$

5 minutes

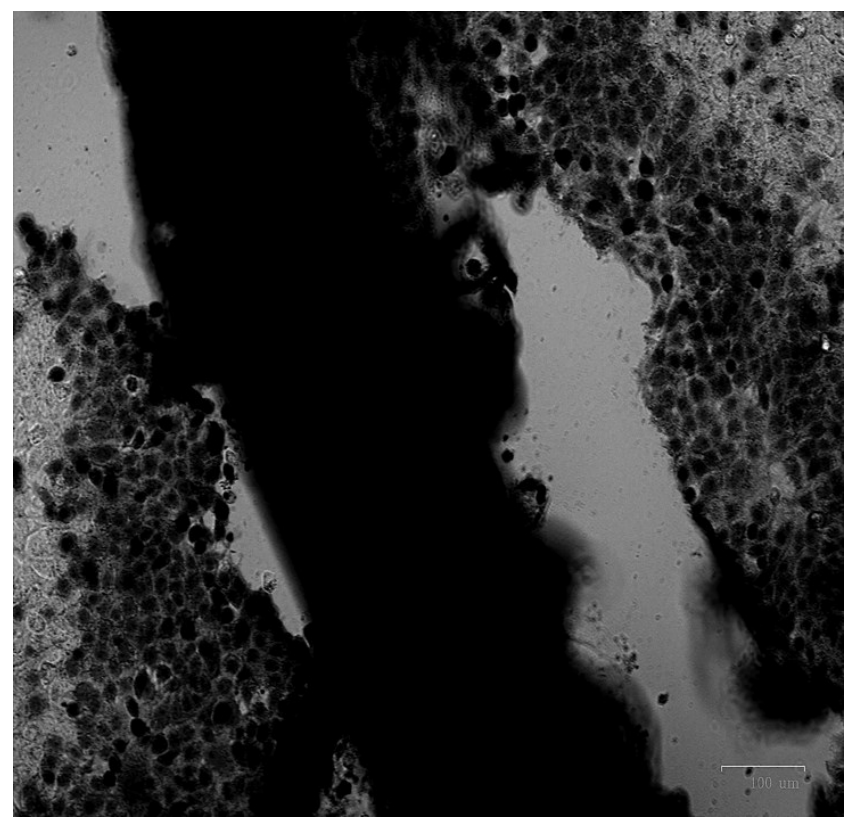

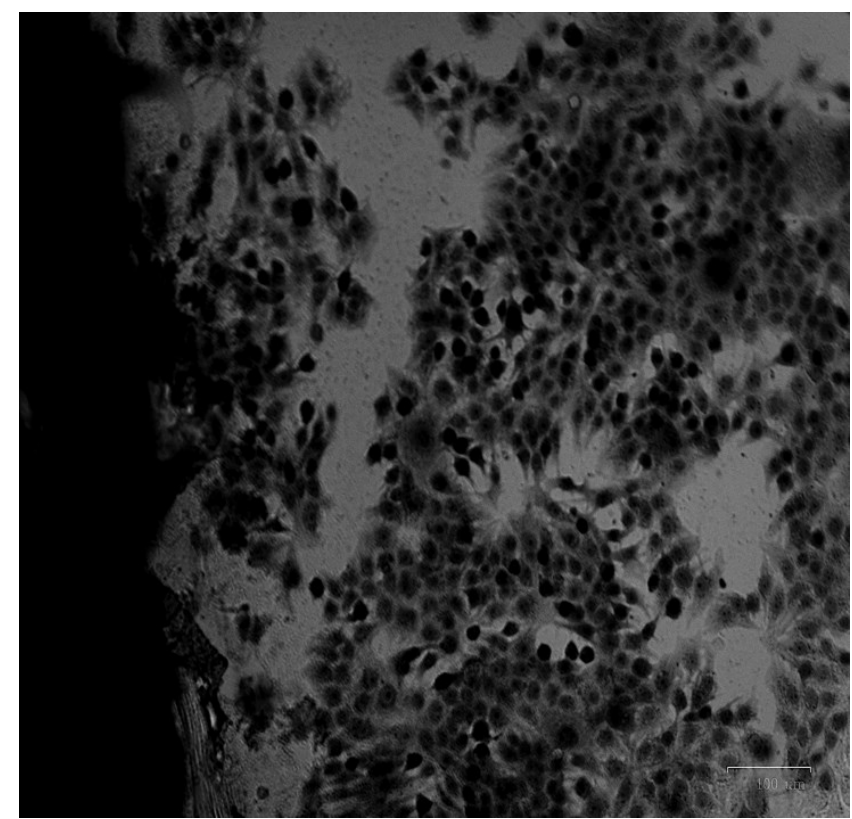

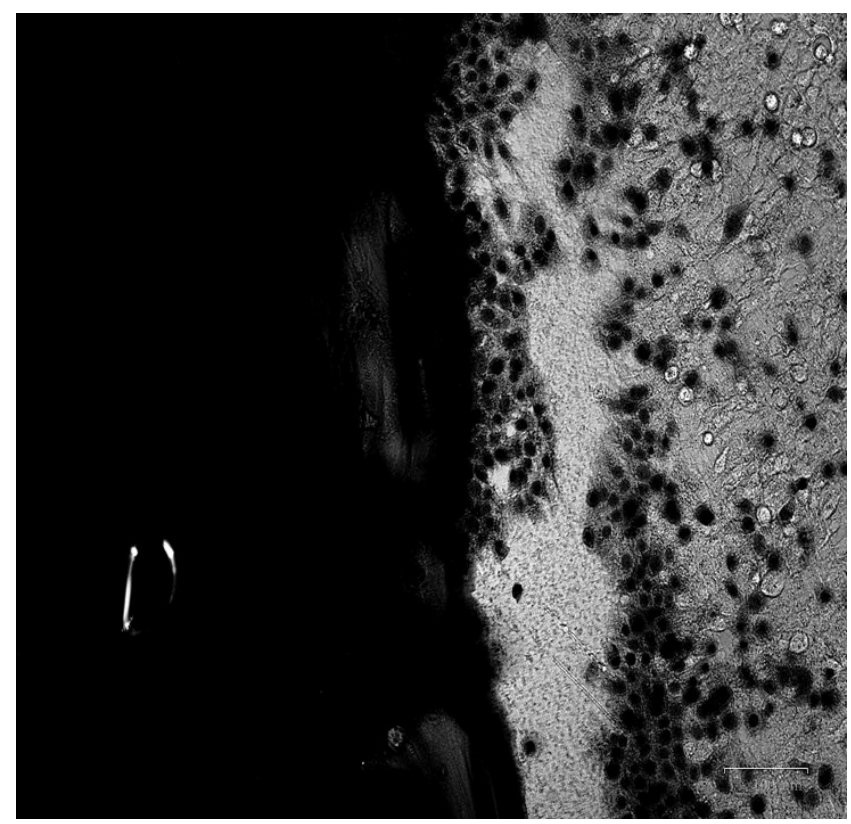



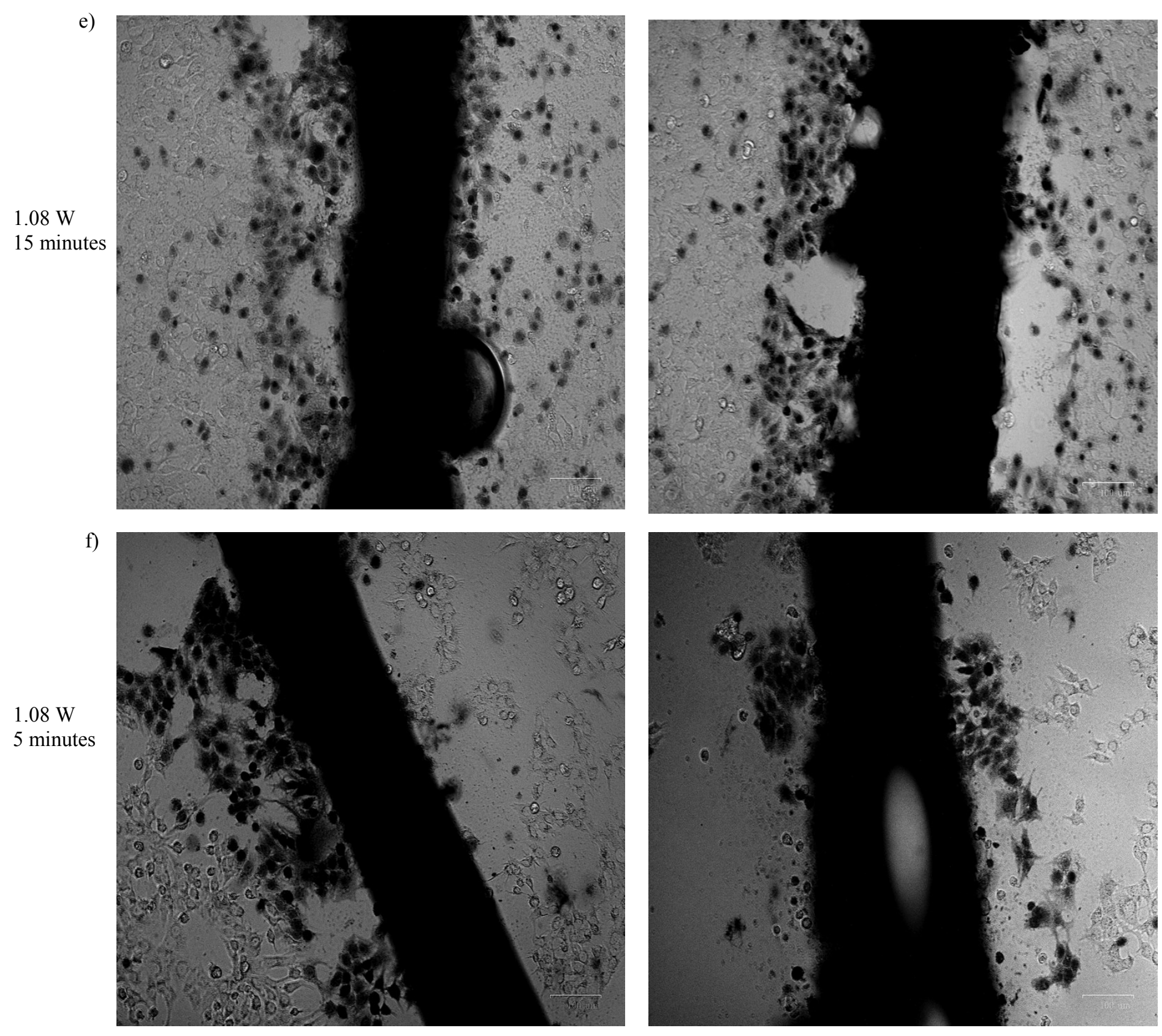


\begin{tabular}{c|c|c|c|c} 
Image & $\begin{array}{c}\text { Treatment time } \\
\text { (minute) }\end{array}$ & $\begin{array}{c}\text { Temperature } \\
\text { at the active } \\
\text { heater surface }\end{array}$ & $\begin{array}{c}\text { Diameter of } \\
\text { the killing } \\
\text { zone }\end{array}$ & $\begin{array}{c}\text { Temperature at } \\
\text { outer points of } \\
\text { the killing zone } \\
\text { radius }\end{array}$ \\
\hline a & 15 & $66.02{ }^{\circ} \mathrm{C}$ & $1696.408 \mu m$ & $45.02{ }^{\circ} \mathrm{C}$ \\
\hline b & 5 & $62.013^{\circ} \mathrm{C}$ & $1494.101 \mu m$ & $44.641{ }^{\circ} \mathrm{C}$ \\
\hline c & 15 & $57.74{ }^{\circ} \mathrm{C}$ & $1318.101 \mu m$ & $43.474{ }^{\circ} \mathrm{C}$ \\
\hline d & 5 & $54.4502^{\circ} \mathrm{C}$ & $1209.844 \mu m$ & $42.11^{\circ} \mathrm{C}$ \\
\hline e & 15 & $50.43{ }^{\circ} \mathrm{C}$ & $820.196 \mu m$ & $42.538^{\circ} \mathrm{C}$ \\
\hline f & 5 & $46.86^{\circ} \mathrm{C}$ & $468.368 \mu m$ & $42.77^{\circ} \mathrm{C}$
\end{tabular}

Figure 5.6. MCF7 cells viability as a function of pump power and time. 



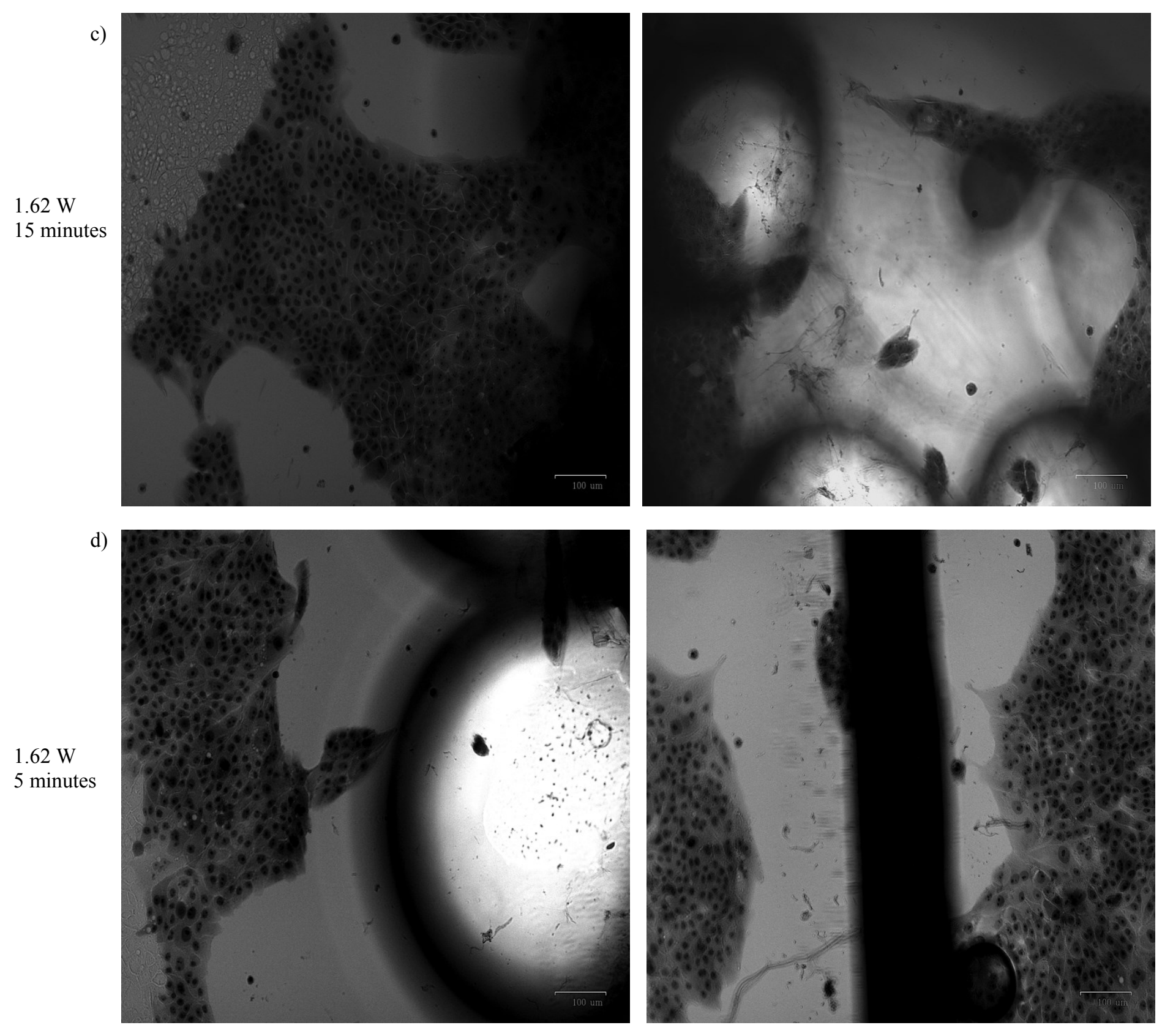

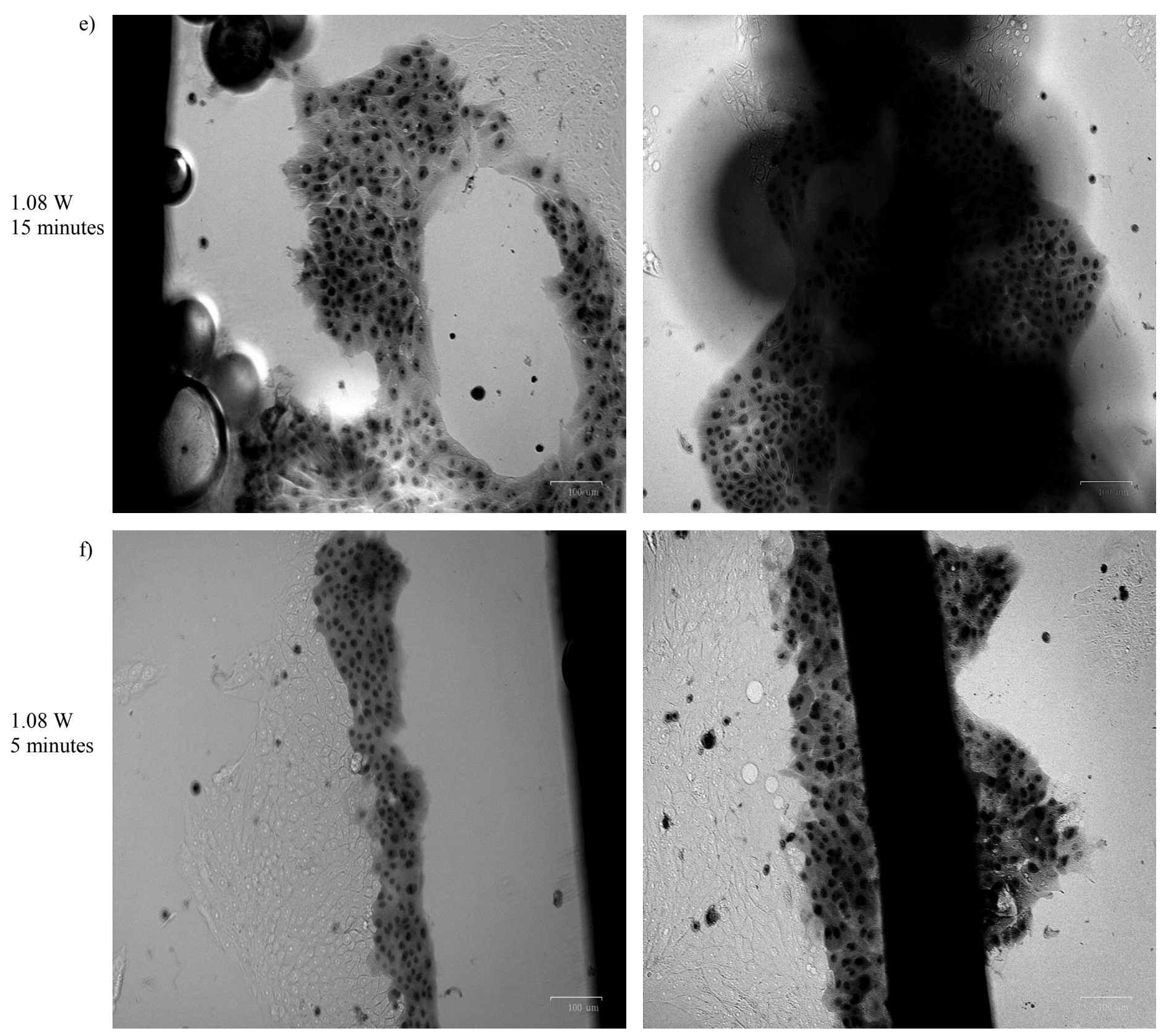


\begin{tabular}{c|c|c|c|c} 
Image & $\begin{array}{c}\text { Treatment time } \\
\text { (minute) }\end{array}$ & $\begin{array}{c}\text { Temperature } \\
\text { at the active } \\
\text { heater surface }\end{array}$ & $\begin{array}{c}\text { Diameter of } \\
\text { the killing } \\
\text { zone }\end{array}$ & $\begin{array}{c}\text { Temperature at } \\
\text { outer points of } \\
\text { the killing } \\
\text { zone's radius }\end{array}$ \\
\hline a & 15 & $66.52{ }^{\circ} \mathrm{C}$ & - & - \\
\hline b & 5 & $62.2476^{\circ} \mathrm{C}$ & $1863.263 \mu m$ & $40.515{ }^{\circ} \mathrm{C}$ \\
\hline c & 15 & $57.2476^{\circ} \mathrm{C}$ & $1615.656 \mu m$ & $39.922{ }^{\circ} \mathrm{C}$ \\
\hline d & 5 & $52.5238^{\circ} \mathrm{C}$ & $1173.192 \mu m$ & $40.98^{\circ} \mathrm{C}$ \\
\hline e & 15 & $50.5952{ }^{\circ} \mathrm{C}$ & $1040.262 \mu \mathrm{m}$ & $40.72{ }^{\circ} \mathrm{C}$ \\
\hline f & 5 & $47.13{ }^{\circ} \mathrm{C}$ & $761.3 \mu \mathrm{m}$ & $40.41{ }^{\circ} \mathrm{C}$
\end{tabular}

Figure 5.7. MCF-10A cells viability as a function of pump power and time. 
a)

c)
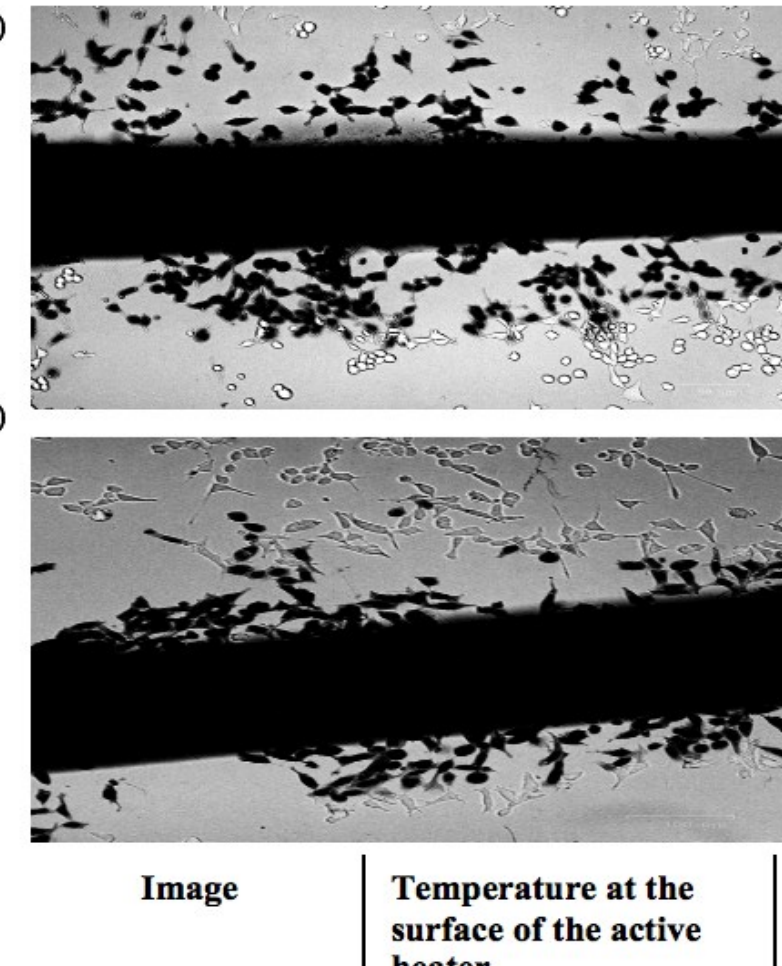

\begin{tabular}{l|l}
\multicolumn{2}{|l}{ heater } \\
\hline a & $44.62^{\circ} \mathrm{C}$
\end{tabular}

\begin{tabular}{c|c|c|c}
\hline b & $43.84^{\circ} \mathrm{C}$ & $401.409 \mu m$ & $40.43^{\circ} \mathrm{C}$ \\
\hline c & $43.4857^{\circ} \mathrm{C}$ & $407.53 \mu m$ & $40.17^{\circ} \mathrm{C}$ \\
\hline d & $42.87^{\circ} \mathrm{C}$ & $356.494 \mu m$ & $40.008{ }^{\circ} \mathrm{C}$
\end{tabular}

Figure 5.8. HEK 293 cells viability as a function of pump power and time.

To confirm the reliability of our measurements, we examined the effect of a close range

of temperatures $\left(43.8,42.8,44.6\right.$, and $\left.43.2^{\circ} \mathrm{C}\right)$ on HEK293 cells. Table in figure 5.8. displays the total length of the killing zone across the active heater. The length of the killing zone is proportional to the amount of heat produced by the active heater. Although HEK293 cell deaths are confined around the active heater with a minimum toxic temperature in order of $40^{\circ} \mathrm{C}$, this instant cell death is considered significant given the short treatment time of 5 minutes. Unlike bulk heating, localized hyperthermia can cause 
cell death at these temperatures, even when applied for only a short time $[61,217,218]$.

In case of bulk heating, cellular deaths in HEK293 cells can be observed after 48 to 72 hours post-exposure to $42{ }^{\circ} \mathrm{C}$ for 20 - 90 minutes. Minimum temperatures ranging from 39.5 to $40{ }^{\circ} \mathrm{C}$ are reported to cause upregulation in cyclinD1 and $\mathrm{Cdc} 25 \mathrm{~A}$ and hence cause stress in HEK293 cells.

Figures 5.9 compares the viability of the two breast cell lines, one non-cancerous (MCF10A) and one cancerous (MCF7), after undergoing hyperthermia for 5 and 15 minutes.

Viability was determined by counting dead cells fractions relative to those measured from control culture plates not exposed to heat. We exposed MCF-10A and MCF7 cells to continuous heat produced by a range of pump powers $(1.8,1.5$, and $1 \mathrm{~W})$ for 5 and 15 minutes. The results show that MCF7 cells are more resistant to heat than MCF-10A

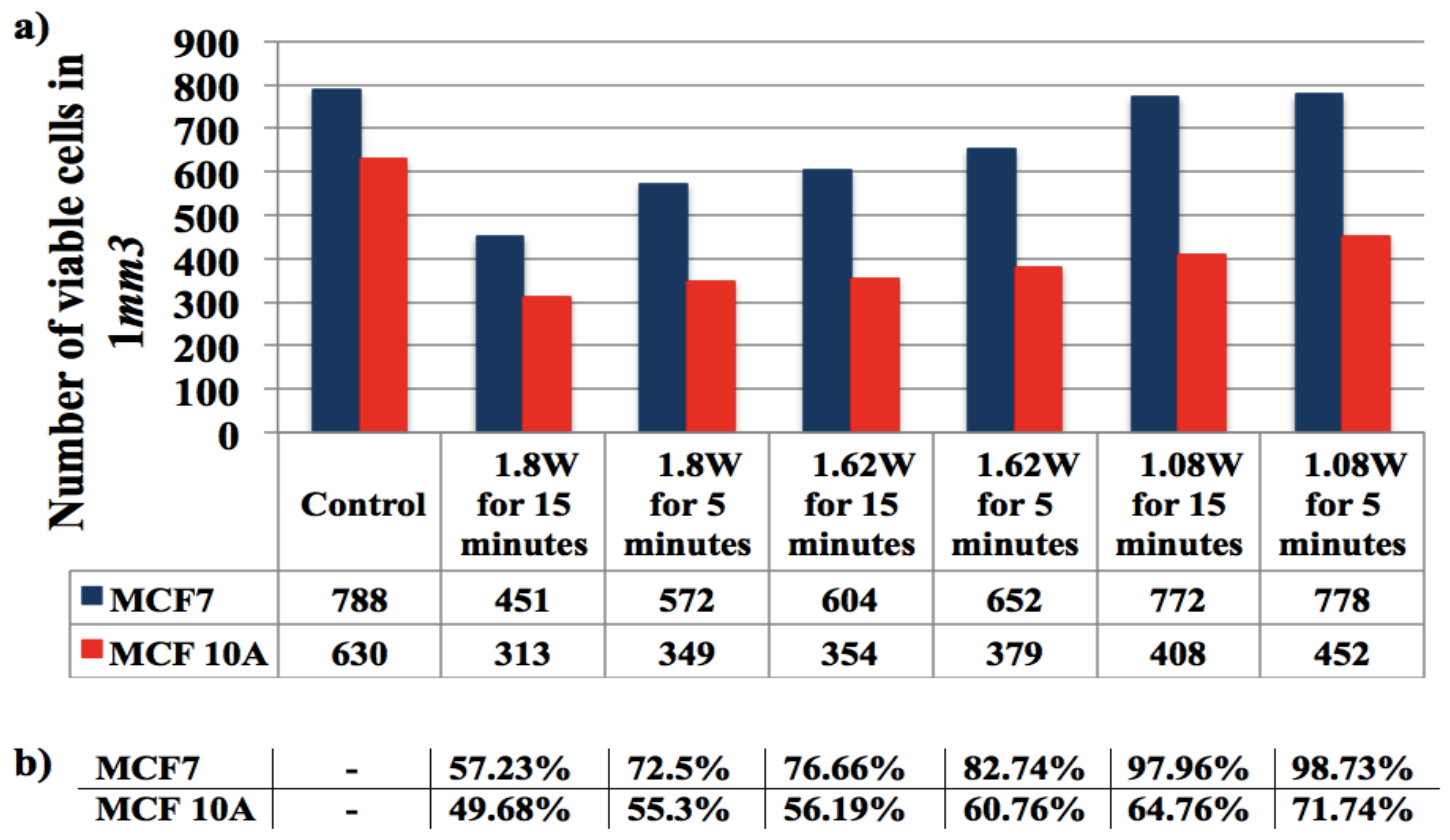

Figure 5.9. (a \& b) Survival cells rate as a function of power and time; MCF-10A and MCF7 cells viabilities were normalized to cells grown under the same condition without exposure to hyperthermia. 
cells, with survival rates between 8 and 33\% better for the tests conditions presented. On the other hand, increasing the treatment durations from 5 to 15 minutes did not lead to important differences in cell viability (no more than about 5-7\%) except for the $1.8 \mathrm{~W}$ treatment of the MCF7 cell line. The low-thermal sensitivity of MCF7 cells, which we encountered, supports the findings of other works when compared to other breast cell lines, MCF-10A cells and MDA-MB-231 breast cancer cells [61], or other cell lines including HEK293 [218].

Some of these considerations are further supported by microscope images of the corresponding cell culture dishes after treatment, as shown in Figure 5.6, 5.7 and 5.8 The stained nonviable MCF-10A cells are surrounded by a necrotic liquid while the stained nonviable HEK293 and MCF7 cells are not. This indicates the cellular death in MCF10A cells is caused by colliquative necrosis. Parts of the cells are packaged up and come off due to the rupturing of the cell membranes. Thus, heat causes MCF-10A cells to die and release the cells' contents, as can be seen by the clear line between viable MCF-10A cells and stained nonviable MCF-10A cells. In contrast, the heat causes stress in HEK293 and MCF7 cells leading to controlled cell death from apoptosis (no cell membrane rupture). 


\section{3 Hyperthermia of Egg White Phantom, Gelatine Phantom and Ex Vivo Hyperthermia of Porcine Liver:}

For Gelatine-based phantom, which made from the jelly powder as described in

Chapter.4, the heat was induced in the Gelatine-based phantom causing a temperature

increase of $120 \mathrm{C}$. However, it was hard to acquire the shape and the dimension of the damaged volume because of the elasticity of the jelly.

Figure 5.10. illustrates the cross sections of the lethal volume of the egg white phantom and the ex vivo liver exposed to hyperthermia for 10 minutes at $1.8 \mathrm{~W}$. The lethal localized diameters for the egg white phantom and the ex vivo porcine liver were similar with lesions of about; $5 \times 12 \mathrm{~mm}$ in the egg white phantom and $7 \times 15 \mathrm{~mm}$ in the porcine

5 minutes

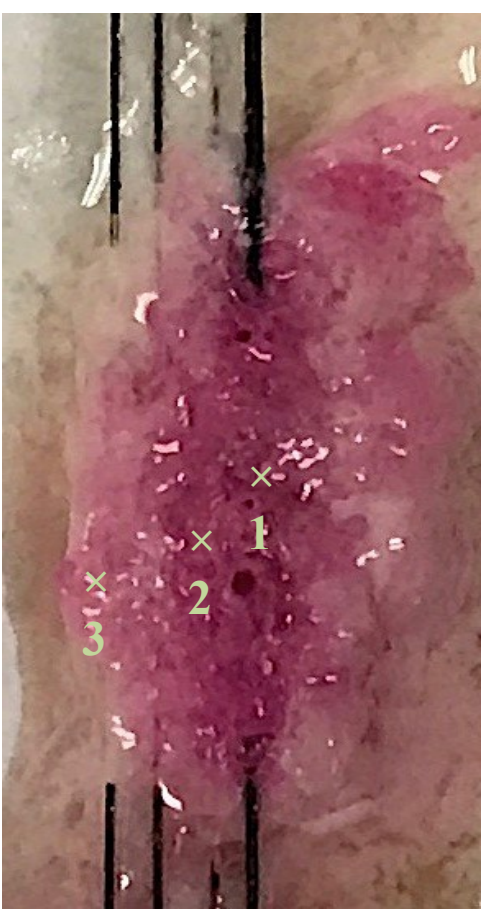

10 minutes

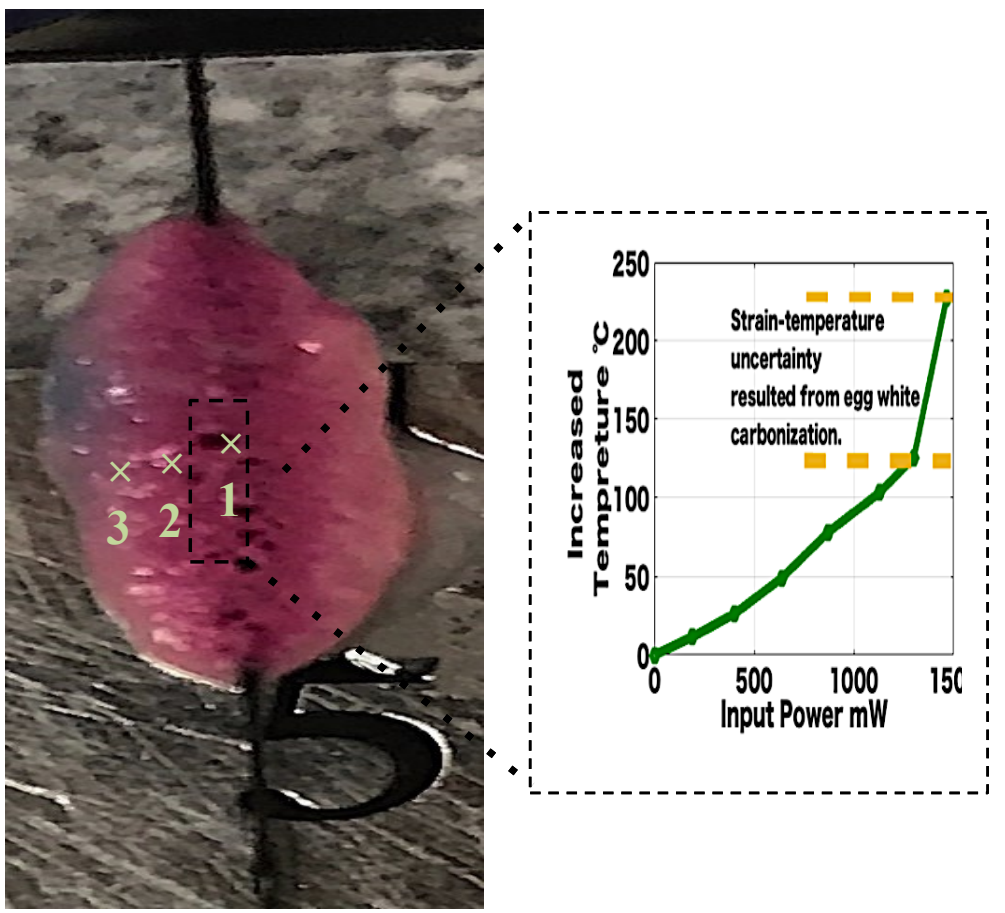

Figure 5.10. The degree of the thermal damage as a function of heating time at a fixed power, and qualitative assessment of temperature gradients in correspondence to the color change of the ink.; 3 temperature regions represented by the symbol $(\times):(\times 1) 139^{\circ} \mathrm{C},(\times 2) 70^{\circ} \mathrm{C}$, and $(\times 3) 50{ }^{\circ} \mathrm{C}$. 
liver resulted from 10 minutes of continues heating and $4.6 \times 10 \mathrm{~mm}$ in the egg white phantom and $6 \times 13 \mathrm{~mm}$ in the porcine liver resulted from 5 minutes of continues heating. Figures 5.10. and 5.11 show the damage caused by the active heater in the egg white phantom and ex vivo porcine liver. Continuous heating at high therapeutic temperatures does not cause a significant increase in the lethal volume. The cumulative effect of the continuous flow of heat can be clearly seen in the phantom as the egg white turns into a white hard lesion. In the ex vivo liver, as the active heaters keep generating heat, the ex vivo liver tissues close to the active heater get burned and stick to the active heater.

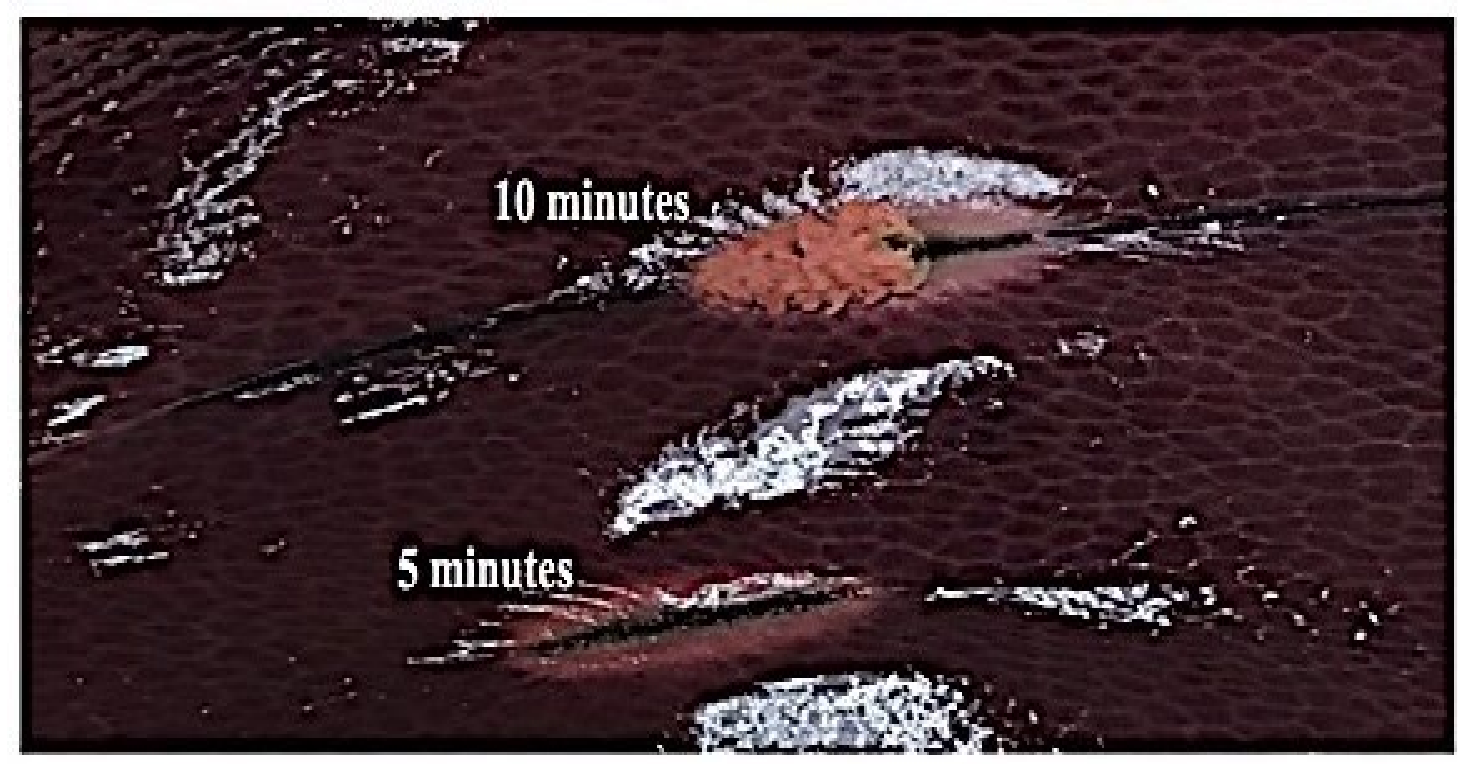

Figure 5.11. The degree of the thermal damage as a function of heating time at a fixed power in ex vivo porcine liver.

These differences in the lesion dimensions between the egg white and the porcine liver are to be expected. The heat generated by the active heater decreases as the distance from the active heater is increased. In other words, the build-up temperature increase at $2 \mathrm{~mm}$ away from the heater is very slow, thereby allowing the natural convection in the egg white to occur. The density of the egg white reduces as the egg white temperature 
increases. Thus, the natural convection disturbs the slow build-up temperature in the egg white at a farther distance from the active heater. On the other hand, at a closer distance to the active heater intense heat is generated in a very small volume for a very short period of time. This causes intense thermal denaturation to the egg white closed to the heat source, an effect which was observed experimentally within 0.23 minutes of starting the heating process at $1.8 \mathrm{~W}$. The extreme dependence of the egg white protein denaturation on the temperature increase is explained well in the literature [219-223]. As stated in the literature, the thermal denaturation of such protein occurs 600 times faster when the protein temperature has been increased by $10^{\circ} \mathrm{C}[220]$.

We also performed qualitative assessment of temperature increase based on visual colour change of the egg white mixed with thermochromic ink (Figure 5.10). Based on the visual colour change of the thermochromic ink from white to pink and magenta, it was possible to estimate two temperatures regions with a radius of $0.620 \mathrm{~mm}$ with $50{ }^{\circ} \mathrm{C}$ and $0.758 \mathrm{~mm}$ with $70{ }^{\circ} \mathrm{C}$, respectively. The temperatures of the colours were determined by comparing the two visible changes in the phantom colours to the vial colours associated with known temperatures as shown in Figure 4.9. The magenta colour, which associated with an approximate temperature of $70^{\circ} \mathrm{C}$, at the active heater surface was not considered because of the ink colour saturation at this temperature. Instead, the Bragg response shift was used to determine the temperature increase of $139^{\circ} \mathrm{C}$ at the active heater surface (Figure 5.10). 


\section{3. 1 Hyperthermia-induced Lesion in Egg White Phantom by the Packaged TFBG-based Active Heater:}

Figures 5.12 and 5.13 show the amount of lesions caused by packaged TFBG-based active heater. These packaged devices consist of TFBG emerged in amorphous carbon pigments. Figure 5.12 illustrates the heating profile and temperature monitoring of continuous heating at $1.4 \mathrm{~W}$ for 10 minutes using the packaged dual-purpose TFBG in the egg white phantom. We also observed localized carbonize layers in the egg white lesion attached to the needle surface. The slow heat flow compared to the speed of heat dissipation in the egg white results in a very high increase, in order of $180^{\circ} \mathrm{C}$, to the temperature of the needle surface.

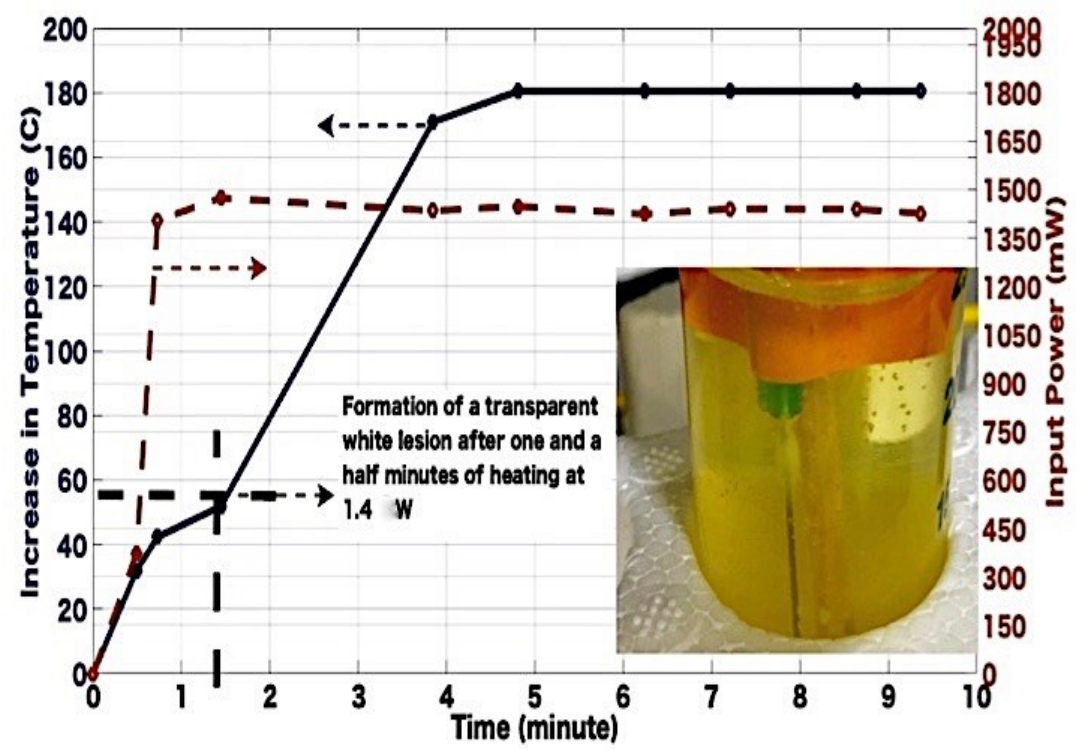

Figure 5.12. TFBG-based active heater enclosed in the needle inside the fresh hen egg white causing a lesion size in order of $7.9 \times 14.5 \mathrm{~mm}$.

Figures 4.4 and 5.13 also show the heating efficiency of bare TFBG and the packaged device. As we have stated that the evaluation of the heating efficiency of our devices are performed in $45 \mathrm{ml}$ water contained in the test tube and placed in the oven with a temperature setting of $22^{\circ} \mathrm{C}$. It seems that the heating efficiency acquired from the 
coated TFBGs is high compared to the heating efficiency obtained from the packaged device.

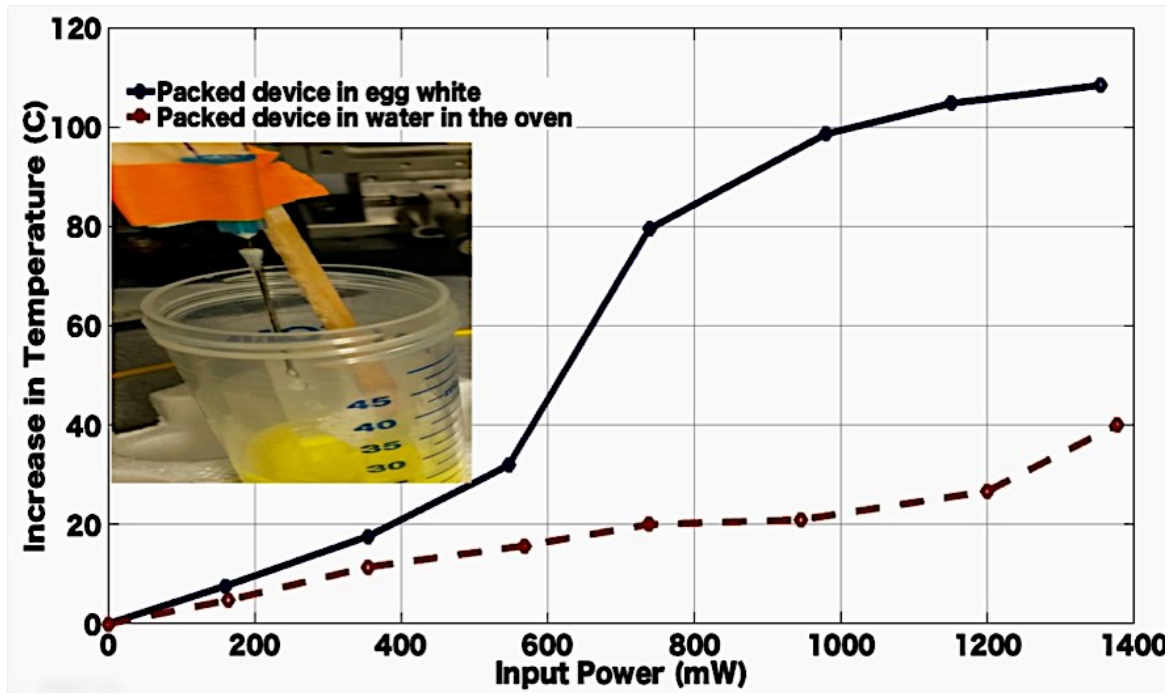

Figure 5.13. Lesion size in order of $7.583 \times 5.24 \mathrm{~mm}$ and the increase in temperature measured by the packaged TFBG-based active heater and the temperature increase in correspondence to Bragg resonance shifts.

\section{4 Thermal Stability of Fs-FBGs:}

Figure 5.14. shows the room temperature transmission spectrum of the first order grating before and after the regeneration process and the transmission spectrum of a third-order grating at room temperature before and after annealing. 

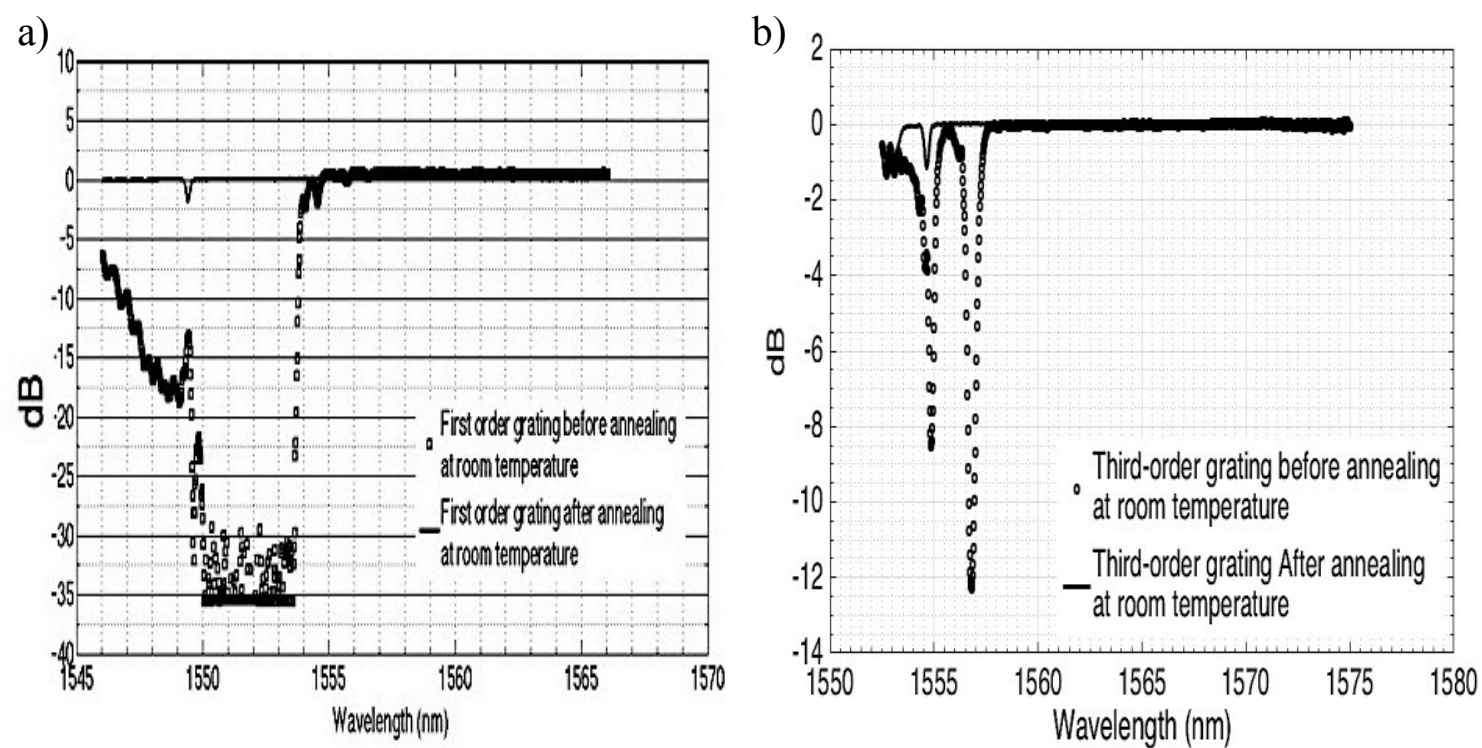

Figure 5.14. Transmission spectrum at room temperature of the (a) first-order grating before and after the regeneration process, and (b) third-order grating before and after annealing

In Figures 5.15 and 5.16, we compare the short- and long-term temperature stability of regenerated fiber Bragg gratings made with a first order phase mask and higher order gratings formed with a third order phase mask. The initial index change of the gratings was determined by matching the spectral bandwidth and transmission loss using Rouard's method [248]. When calculating the induced index change for the $3^{\text {rd }}$ order devices, we assumed a first order resonance and a sinusoidal grating profile. This initial value was then used to normalize the remainder of the data. For the first order devices the spectral response saturated the detector until roughly $750{ }^{\circ} \mathrm{C}$ (as the device is measured in transmission). For this reason, the normalized index change has been set to 1 for these temperatures, even though they would be lower if they were measurable. Over long-term annealing, we observe that the regenerated devices tend to be more stable than their $3^{\text {rd }}$ order counterparts (which are written with similar conditions). 


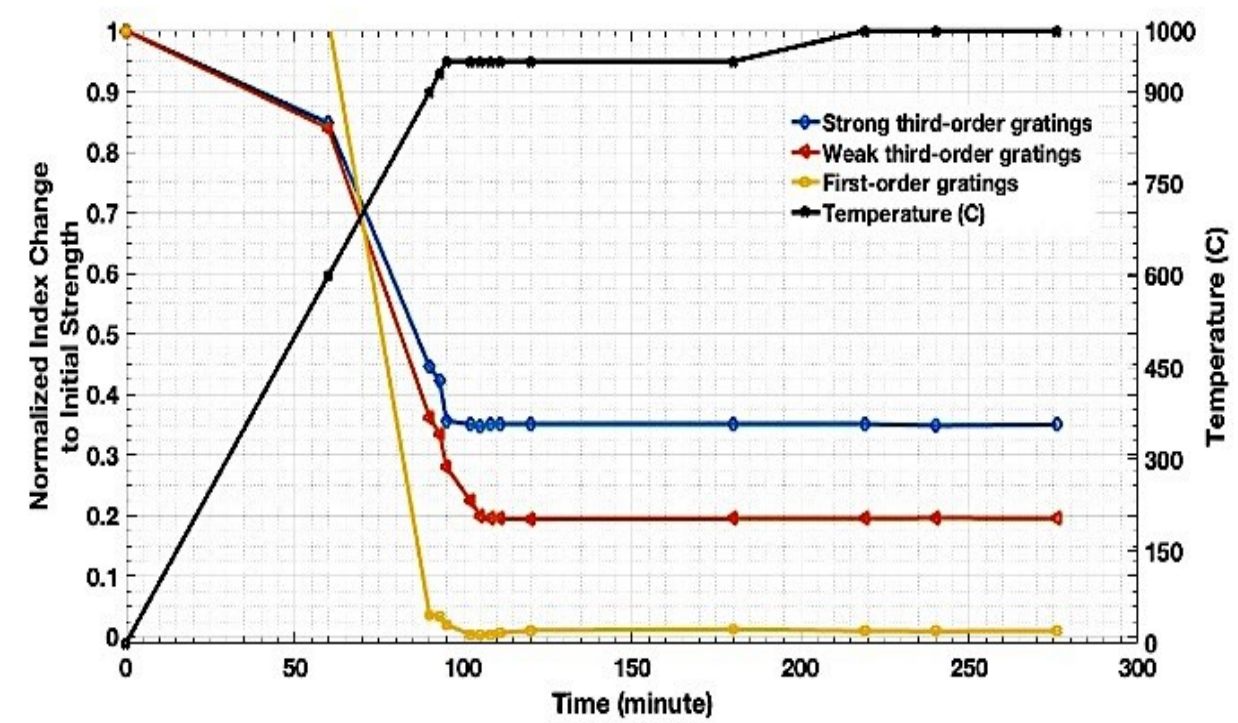

Figure 5.15. Comparison of $1^{\text {st }}$ and $3^{\text {rd }}$ order grating's normalized index change during the short term annealing.

The short- and long-term annealing behaviour is a measure of the evolution of the stressbased grating in the fiber. It is possible for the first order device to appear to anneal out while maintaining a significant signal at the third order that disappears as the device smooth's out at high temperatures. The perceived short-term stability of the third order device could be related to the longer duration over which the third order fourier component of the device disappears at high temperatures. It is likely that the grating is smoothed out and resembles a sinusoidal index profile as the grating anneals, resulting in the elimination of higher order components. 


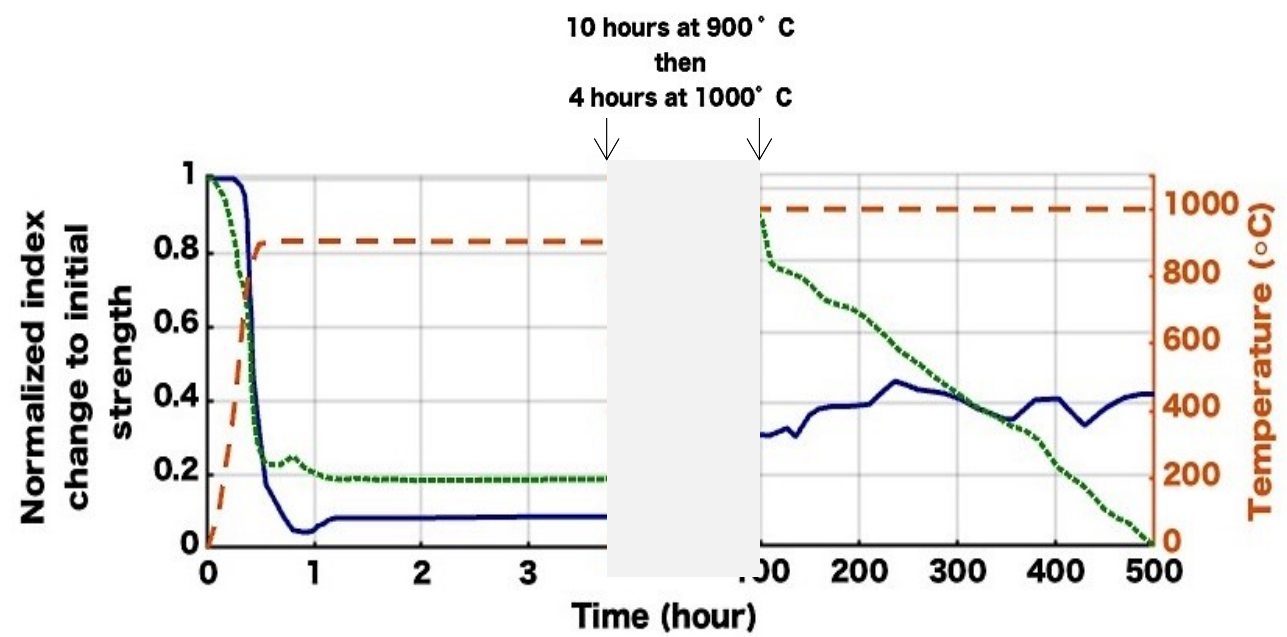

Figure 5.16. Comparison of $1^{\text {st }}$ (blue) and $3^{\text {rd }}$ (green) order grating's normalized index changes during the long-term annealing.

The first and third order devices were inscribed with similar initial conditions, suggesting that the annealing behaviour would be similar in each case. This is clearly not the case from the data shown in Figure 4.12. In previous work we saw that the growth behaviour of $3^{\text {rd }}$ order gratings behaved very differently from the first order devices as well [70, $249,250]$. We concluded that the difference could be attributed to the fact that the $3^{\text {rd }}$ order device behaviour is a measure of the $3^{\text {rd }}$ order fourier component of the grating. 


\section{Chapter 6: Mathematical Modelling}

This chapter describes a numerical simulation we built to enable the estimation of the thermal damage associated with the temperature increase caused by the active heater in the thermal lesion region during hyperthermia by using the reading temperature of the active heater surface. We use the experimental setup (three different powers $1 \mathrm{~W}, 1.2 \mathrm{~W}$ for 5 minutes, and $2 \mathrm{~W}$ for $5 \& 10$ minutes) and observation (at which time we started to see the lesion formation) as a base for the simulation input data to validate our simulation (by matching the lesion dimensions and the temperature increase at the active heater and at different distances). The objective for considering such theoretical evaluation is to model the influence of the TFBG-based heater in the localized thermal therapy under the presence of perfusion rates, hence, determination of the device's ability and limitation.

\section{1 Introduction:}

COMSOL is a computational software used to simply transfer a physical problem into a mathematical model. This helps to comprehend and acknowledge the physical problem and then look for solutions to optimize the system and make it more efficient. Computational software also has a significant impact on demonstrating the potential of new devices through simulating their performance in a controlled medium that is parallel to a medium in which it is difficult to perform experiments such as the human body. This helps to reduce the amount of testing on animals and the effort required in several therapeutic practices including hyperthermia and cryosurgery. Given the fast progress in thermal modelling, computational software has good potential to be used clinically in hyperthermia treatment planning [251-255]. Thermal models may help to overcome the insufficient temperature measurements obtained from only discrete points in the treated 
volume. Thus, providing physicians with the temperature distribution throughout the target volume will allow for better quantification to the delivered thermal dose.

The finite element method (FEM) is the numerical technique used in COMSOL to find approximate solutions to a set of partial differential equations (governing equations) which are subjected to sets of equations describing boundary conditions [256, 257]. The solution of the governing equations is usually divided into a discrete set of sharply defined regions and points known as elements and nodes. These elements are usually created by using a mesh in the software. Within each element, the approximate solution of dependent variables including temperature, velocity and pressure are found as a function of time or space.

In this study, we use COMSOL to simulate the temperature gradient of the heat conduction transfer shape created around the active heater as a function of time. In local hyperthermia cancer treatment, the induced temperature within a tumor of a specific volume decreases in a radial direction away from the applicator $[5,87,126-128]$. The reduction rate of the simulated temperature depends on the type of tumor and the type of heating source. The heat penetration depth is calculated so that the amount of the power or temperature is reduced to $1 / \mathrm{e}$ of that on the surface of the applicator.

We aim to provide a numerical model that is able to predict the degree and the shape of the lethal damage by observing and monitoring the raise in active heater surface temperature. The temperature increase in the three-dimensional domain described by the bioheat transfer equation is solved by the FEM method. The transient temperature distribution caused by the conductive heat transfer is found within: (1) an egg white phantom; and (2) porcine and human liver with or without considering the effect of blood 
perfusion. We then compare the simulated results to the experimental lethal damage obtained in an egg white phantom and in ex vivo porcine liver.

\section{2 Bioheat Transfer Model:}

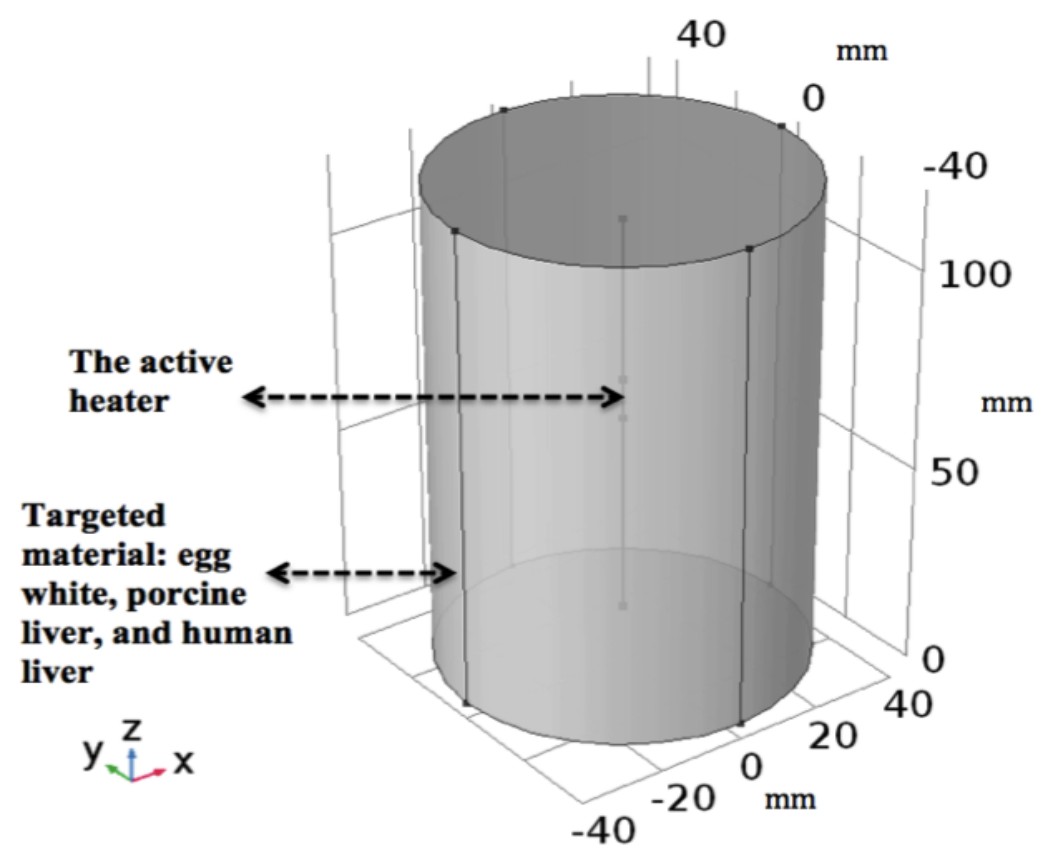

Figure. 6.1. Geometrical shape of the simulated material

Figure 6.1. indicates the geometrical shape used in the model. The geometry of the targeted material was designed as a 3D cylinder with the active heater in the middle. This module consists of three domains: the grating domain, the fiber domain, and the materials domain. In the materials domain, we used three materials in this simulation: egg white, porcine liver, and human liver. We switch between these materials to simulate the heat transfer in each material under similar conditions, such as heating power and time. The active heater is simulated as a hot wire produces heat in a control volume. This heater is in direct contact with the materials domain.

The only mode of heat transfer (heat source) within the materials is caused by heat conduction. The simulation gives a solution for the governing equation to provide a 
transient analysis of the temperature gradients increase caused by the flow of heat from the active heater surface into the heated target. The total process time was around 2 hours and 21 minutes for the egg white and around 7 minutes for both human and porcine livers with/without blood perfusion. The time steps were set in arrangements of $(0$, step by 1

second, 1 minute) and (1-minute, step by 120 seconds, 20 minutes). The selection of time steps and the mesh size were determined by trying different values as the ability of the used PC allowed. The maximum relative error was left in the default setting of the software in order of $10^{-3}$. This relative tolerance error controls the degree of accuracy in the solution. As its value goes tighter, the solution accuracy increases at the expense of the computational time for a given mesh. In our case, this value is good enough because the inputs (material properties) in our model are approximate values [259-269]. The biological tissues properties vary between individuals, due to different age and health status for example.

\section{2. 1 Creating the Geometry:}

COMSOL covers various types of geometries including 1D and 2D axisymmetric, and $1 \mathrm{D}, 2 \mathrm{D}$, and 3D [256]. Our case is a very simple transient heat transfer problem which can be represented by $2 \mathrm{D}$. However, for better compression to the $3 \mathrm{D}$ egg white lesion shape obtained experimentally, we used a 3D computational domain. Two major techniques are available in COMSOL to create the 3D geometries for a physical problem: (1) a bottom-up approach which starts with 2D geometries and uses extrusion or revolution tools to bring the $2 \mathrm{D}$ into $3 \mathrm{D}$ shapes; and (2) a top-down approach in which a 3D shape such as a cylinder, block, or cone is chosen directly. 
In our models, we used the following two approaches to create the two concentric cylinders: the heating material and the active heater (Figure 6.1). The heating material is represented by a cylinder with height and diameter of $12 \times 4 \mathrm{~cm}$. The $10 \mathrm{~mm}$-long active heater shaped as a cylinder with length and radius of $100 \mathrm{~mm}$ and $0.235 \mathrm{~mm}$ was designed in the geometric centre. Table 6.1 shows the steps taken to create the geometry. After creating the geometries, we divide the computational domain (geometries) into subdomains (as shown also in Table 6. 1). Each subdomain has its boundaries which the user can apply to help specify conditions between the subdomains.

\begin{tabular}{|c|c|}
\hline Geometry & $\begin{array}{l}\text { Go to Geometry>>create Work Plane } 1 \text { (wp1): } \\
\text { Choose the plane type (quick). } \\
\text { Go to wp1's plane geometry >>choose Circle 1(c1) and Circle } \\
\text { 2(c2): } \\
\text { Specify the radius of (c1) and (c2) as } 0.235 \mathrm{~mm} \text { and extrude (c1) by } 10 \\
\text { and then by } 50 \mathrm{~mm} \text { and check the pox for unit with input objects and } \\
\text { (c2) by - } 50 \mathrm{~mm} \text {. These are the dimensions of the active heater in the } \\
\text { middle of SMF28 fiber. } \\
\text { Go to geometry >>choose cylinder } 1 \text { (cyl1): } \\
\text { Specify the radius and the height of cyll as } 40 \mathrm{~mm} \text { and the height } 120 \\
\text { mm and choose its axis (z-coordinate). }\end{array}$ \\
\hline Subdor & $\begin{array}{l}\text { Domain } \# \mathbf{1}>>\text { materials domain; its boundary: exterior boundary at } \\
(1-4,15 \text {, and } 22) \\
\text { Domain } \# \mathbf{3}>>\text { grating domain; its boundary at }(8-13,17,18,20 \text {, and } \\
21) \\
\text { Domain } \# \mathbf{2} \mathbf{\&} \mathbf{4}>>\text { fiber domain; their boundary at }(5-7,14,16 \text {, and } 19)\end{array}$ \\
\hline
\end{tabular}

Table 6.1. Geometries and subdomains created in the model.

Figure 6.2 shows the types of meshes and their properties [256]. The meshing was generated in unstructured "Normal" free tetrahedral type, with maximum and minimum element sizes of $0.38 \mathrm{~mm}$ and $0.35 \mathrm{~mm}$, respectively, in the grating domain; and $1.3 \mathrm{~mm}$ and $1.1 \mathrm{~mm}$, respectively, in the rest of the geometric domains. This unstructured mesh type is very flexible with an automatic ability for rapid grading from small to large 


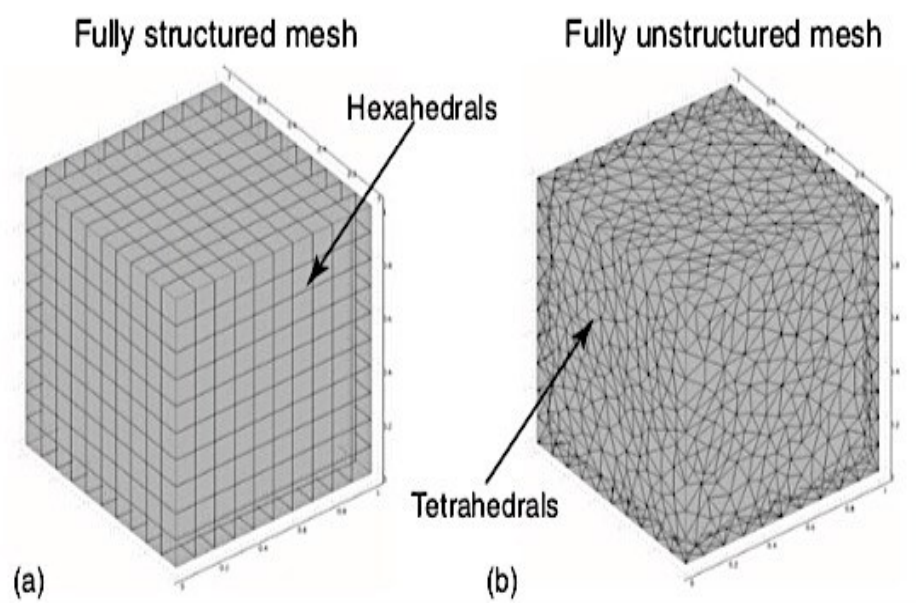

Figure. 6.2. Types of mesh in COMSOL: (a) Structured mesh can be used for simple geometries. Also, the number of nodes and elements has to be equal: (b) Unstructured mesh gives high flexibility in designing complex geometries [256].

elements. Thus, we used this mesh to simulate the volume within which the largest changes occurred with good precision and short computational time. We started meshing with a finer mesh structure at and around the grating domain where the temperature changes at the grating surface happen very fast within a few seconds of starting the heating process based on the experiment observations. The total counts of trigonal elements were 85416 .

\section{2. 2 Governing Equation:}

A mathematical model designed to describe heat transfer in a living biological system must consider the heat transfer within two phases: solid phase and liquid phase [258, 276284]. The living tissue portrays the solid phase which is filled with blood vessels. Blood flow within the tissue, in the liquid phase, has a significant effect on the heat transfer processes. The complexity arises when dealing with the effect of blood on the heat transfer within the tissue from its compound vessels anatomy. Blood flows at different speeds due to size disparities of the blood vessels and with different orientations. Further, the blood which flows in vessels with diameters larger than $500 \mu m$ is not in thermal 
equilibrium with its surrounding tissues as it is usually assumed. The heterogeneity structures of the tissues along with the human's age and health also affect tissues thermal properties. These biological factors make the thermal properties including thermal conductivity and specific heat of tissue vary considerably over short distances.

Accordingly, various models have been developed to describe heat transfer and fluid flow in biological systems. Some of these mathematical models are Pennes' bioheat transfer equation [278, 294, 295], Chen and Holmes (CH) model [279] and Weinbaum, Jiji, and Lemons (WJL) model [280-283]. The Pennes bioheat transfer equation is the first quantitative mathematical formulation that quantifies heat transfer in human tissue by including the blood perfusion effect. Following the Pennes bioheat transfer equation, several alternative representations of heat equations were demonstrated. These models were developed to descibe the blood perfusion effect upon heat transfer in living biological tissue.

\section{2. 2. a Prior to Pennes' Work:}

The interest in the relation between the blood perfusion and heat transfer in tissue started in 1876 [278, 284-296]. Before Pennes' finding, the blood perfusion effect on heat transfer was experimentally recognized and formulated as the following equation [278]:

$$
q_{s}=\omega \rho_{b} c_{b} \delta\left(T_{b}-T_{s}\right)+\frac{k_{t}}{\delta}\left(T_{b}-T_{s}\right)
$$

where $q_{s}$ describes surface heat flux from the body to the environment. $q_{s}$ is influenced by: (1) the depth at which the temperature is measured inside the tissue $(\delta) ;(2)$ the tissue thermal conductivity $\left(k_{t}\right)$; (3) the blood properties including perfusion rate $(\omega)$, density $\left(\rho_{b}\right)$, and specific heat $\left(c_{b}\right)$; and (4) the temperature difference between the deep tissue, 
or body core blood temperature such as the rectal temperature, and the skin temperature $\left(T_{b}-T_{s}\right)$

One of the main issues with this mathematical model comes from considering the measurements of heat transfer between the tissue layer and its surroundings obtained from the maximum temperature difference $\left(T_{b}-T_{s}\right)$ and the minimum blood perfusion rate $[278,296]$. Another inaccurate aspect is that the effect of the temperature difference $\left(T_{b}-T_{s}\right)$ extends only to a depth in order of $2 \mathrm{~cm}$. This was questioned by Pennes who formulated the continuous heat transfer relationship between the temperature and the location of the tissue away from the skin by simply using the conduction heat transfer theory [294]. Figure 6.3. shows the temperature gradients distribution from the surface "skin" down to the axis of the arm tissue, which was assumed to take a shape of a symmetrical cylinder in the process of driving the bioheat transfer equation. It is important to mention here that following Pennes' studies, in 1976 Shitzer and Kleiner performed similar studies and showed similar results indicating that blood perfusion dominates heat transfer in deeper tissues [272].

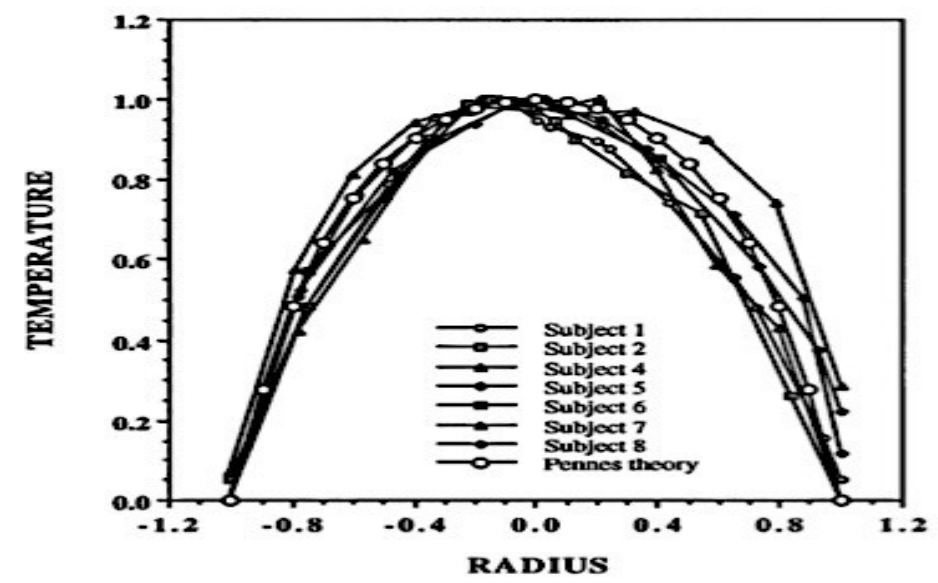

Figure 6.3. Temperature gradients distribution from the surface "skin" down to the axis of the arm tissue: experimental values and the theoretical value [295]. 


\section{2. 2. b Pennes' Bioheat Transfer Equation:}

In 1948, Pennes derived the well-known bioheat transfer equation after measuring the temperatures of the right forearms of human volunteers [278, 294, 295]. The room temperature where the volunteers were placed was kept between $25-27^{\circ} \mathrm{C}$. Also, Pennes used an anemometer to ensure minimal air motion in the room. Skin, deep tissue, and brachial artery temperatures were controlled during the experiment. For the skin temperature measurements, he used a radiometric thermometer and copper-constantan thermocouples. For the deep tissue and brachial artery temperature measurements, thermocouples were inserted through needles. The highest temperatures of the deep tissue were found to be lower than the brachial artery temperatures by an average of $0.16^{\circ} \mathrm{C}$. This finding helped Pennes to formulate his landmark bioheat transfer equation. The bioheat transfer formula is an approximate equation that treats blood perfusion as an isotropic heat source and the main factor responsible for heating the arm tissue [278, 294, 295]. In other words, as the blood at arterial temperature enters the tissue, heat is exchanged between capillary blood and tissue. This behaviour was described by Pennes as the Fick principle which states that the rate of mass transfer between the blood and tissue depends on the rate of blood flow multiplied by the difference between their temperatures. Pennes also assumed that blood flows in the artery with uniform temperatures, in reference to the work of Bazett and McGlone. These two assumptions in the equation leave out the physical theoretical basis by simply treating blood perfusion as a scalar term.

The bioheat transfer equation does not account for the effect of the directionality of blood flow and hence no description of the convective heat transfer mechanism is provided 
[277, 278]. These assumptions have provoked serious contention due to neglecting the physical properties of the blood flowing in human tissue. The main arguments centre on two points: (1) the blood flow is not isotropic; and (2) heat exchange usually happens between counter-current artery-vein pairs. These small vessels not only dissipate heat but also increase heat transfer by conduction through tissues.

This bioheat equation is represented by the following very simple mathematical formula [294]:

$$
\rho c \frac{\partial T_{t}}{\partial t}=\frac{k}{r} \frac{\partial}{\partial r}\left\{r \frac{\partial T_{t}}{\partial r}\right\}+\omega \rho_{b} c_{b}\left(T_{a r t}-T_{t}\right)+Q_{m}
$$

where $\rho, c, k$, and $T_{t}$ are the density, heat capacity, thermal conductivity, and temperature of the tissue. $Q_{m}$ is the rate of metabolic heat generation which was assumed to be uniform. $\omega \rho_{b} c_{b}\left(T_{\text {art }}-T_{t}\right)$ is the blood perfusion term acting either as a heat source or a heat sink depending on its sign in the equation. $\omega$ describes the blood perfusion rate. The values of this term were estimated to be between $1.2-$ and $1.8-\mathrm{ml} \mathrm{blood} / \mathrm{min} / 100 \mathrm{~g}$ tissue in order to fit Pennes' experimental data.

Pennes' work highlights the following important points [278]: (1) as the blood perfusion across the tissue increases, the atrial temperature is the utmost reachable temperature in the tissue (in contrast to the previous overestimation of the tissue temperature by Gagge et al.); and (2) without blood perfusion, the tissue temperature would be higher than its surrounding temperature by 2 to $3{ }^{\circ} \mathrm{C}$ from heat produced during metabolic processes.

\section{2. 2. c Pennes' Bioheat Transfer Equation in Hyperthermia:}

Pennes' bioheat transfer equation is the most common computational model used in studying therapeutic hyperthermia for cancer treatment because it is easy to manipulate and uses common techniques often utilized to solve the heat-conduction equation $[5,87$, 
$122,127,129,130,270,277,278,284,297]$. Furthermore, it provides a realistic estimation of the thermal energy transfer in living tissues. At the capillary level blood flows slowly with a Peclet number smaller than one. Hence, compared to the ratio of heat transfer by conduction, the ratio of convection heat transfer by blood perfusion is insignificant. Thus, conduction is the main mode of transport for the induced heat in the tissues. Table 6. 2 illustrates a general idea of some of the heat transport modes in tissues, bones, blood vessels, and skin. Several successful mathematical models in good agreement with experimental results have applied Pennes' bioheat transfer equation in their investigations $[269,270,292,297-316]$.

\begin{tabular}{llll}
\hline & Conduction & Convection & Radiation \\
\hline Tissues & Significant & Less significant & Insignificant \\
Bones & Significant & Insignificant & Insignificant \\
Blood vessels & Less significant & Significant & Insignificant \\
Skins & Insignificant & Significant & Significant \\
\hline
\end{tabular}

Table 6.2. Heat transport modes in different organs in a living body [251].

Pennes' bioheat transfer equation can also be used to describe heat transfer in cases involving large blood vessels through individual treatment to the effect of blood perfusion in heat transfer [253, 270-316, 311]. For example, Horng et al. described the effect of blood flow in large blood vessels on the induced heat during the hyperthermia treatment of a tumor [316]. They evaluated the temperature gradients' increase in the lesion region by separating the solution for the thermal disruption in the tumor by (1) using Pennes' bioheat transfer equation to describe the temperature increase in the tissues with the effect of microvascular blood perfusion; and (2) using the conventional energy transport equation for blood vessels to describe heat conduction and convection caused by blood flow in large blood vessels. They found that blood with different speed profiles 
including uniform, parabolic, and even pulsatile has no considerable effect on the thermal dose distribution in the target volume. In contrast, a direct influence on the thermal dose efficiency was observed from the blood vessel size and the heating speed on the lesion region. The sensitivity of the thermal therapy to the heating speed was also investigated by Shih et al. who revealed that a long period of hyperthermia treatment suffers more heat loss due to blood flow in large blood vessels in comparison to that of shorter treatment times [296].

In another study, Khanafer et al. concluded the significant effect of blood vessel size on thermal dose [311]. Depending on the treated volume, the blood perfusion effect becomes inconsequential as the size of the blood vessels decreases. Their study also showed that temperature distribution caused by a uniform heating scheme is larger compared to the high temperature increase obtained from a pulsed heating scheme. Thus, a long heating time by the uniform heating technique to achieve a desired therapeutic temperature may cause an overheating volume outside of the target region, thereby, damaging the normal tissue. The authors supported their findings by comparing their numerical model results with the work of others in the literature. The main concern of applying thermal therapy to a tumour that is close to large blood vessels is extending the damage beyond the therapeutic regions, hence injuring the healthy tissues.

\section{2. 2. d Forming the Bioheat Transfer Equation for Our Work:}

We use the active heater to increase a specific volume temperature to therapeutic hyperthermia level. By following Pennes' steps in deriving the bioheat transfer equation along with some modifications to describe our problem, we use the conduction heat transfer theory and treat the blood perfusion as a heat source, "heat sink" [278, 317-321]. 
During hyperthermia, the blood drains the induced heat to balance the tissue temperature. Also, since a large amount of induced heat $(\mathrm{Q})$ is usually applied during hyperthermia, we disregard the heat produced by metabolism.

We consider heat transfer in a control volume along the $\mathrm{x}$-direction [317-321].

Conservation of the thermal energy requires that any increase in the temperature within the control volume should be equal to the amount of the induced heat and the difference between the heat flux coming into and leaving the control volume $\left(q_{x}-q_{x+\Delta x}\right)$. This can be written as:

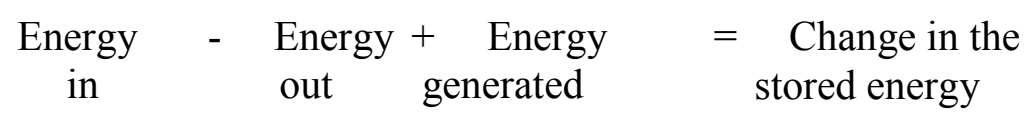

where

Change in the stored energy during time $\Delta t \quad=\Delta x \Delta y \Delta z \rho c \Delta \mathrm{T}$

Energy in during time $\Delta t$ due to conduction $=q_{x} \Delta y \Delta z \Delta t$

Energy out during time $\Delta t$ due to conduction $=q_{x+\Delta x} \Delta y \Delta z \Delta t$

$$
\left(q_{x+\Delta x}\right)
$$

Heat sources; Q energy generated during $=\left[Q-c_{b} W_{b}\left(T-T_{\text {art }}\right)\right] \Delta x \Delta y \Delta z \Delta t \quad$ (6.7) time $\Delta t$ and heat dissipation due to blood perfusion

Thus, generating heat in a control volume at a rate of Q per unit volume causes changes in the stored energy of the heated system $\left(\rho c \frac{\partial T}{\partial t}\right)$ which appear in the volume as temperature rise [317-321]. The energy generation and the change in the volume's stored energy terms are very different. Thermal energy production in the control volume by an external source will cause the temperature to rise in the system. The amount of temperature increase depends on the thermal properties of the heating environment 
including thermal conductivity, density, and heat capacity. These quantities in equations $6.4,6.5,6.6$, and 6.7 are put into equation 6.3 :

$\rho c \Delta \mathrm{T} \Delta x \Delta y \Delta z=q_{x} \Delta y \Delta z \Delta t-q_{x+\Delta x} \Delta y \Delta z \Delta t+\left[Q-c_{b} W_{b}\left(T-T_{\text {art }}\right)\right] \Delta x \Delta y \Delta z \Delta t$

rearranging:

$\rho c \Delta \mathrm{T} \Delta x \Delta y \Delta z=-\left[q_{x+\Delta x}-q_{x}\right] \Delta y \Delta z \Delta t+\left[Q-c_{b} W_{b}\left(T-T_{a r t}\right)\right] \Delta x \Delta y \Delta z \Delta t$

dividing $\Delta x \Delta y \Delta z \Delta \mathrm{t}$

$\rho c \frac{\Delta T}{\Delta t}=\frac{-\left[q_{x+\Delta x}-q_{x}\right]}{\Delta x}+Q-c_{b} W_{b}\left(T-T_{\text {art }}\right)$

letting $\Delta x$ and $\Delta t$ go to zero and using $\lim _{\Delta x \rightarrow 0} \frac{f(x+\Delta x)-f(x)}{\Delta x}=\frac{\partial f}{\partial x}$

$\rho c \frac{\partial T}{\partial t}=-\frac{\partial q_{x}}{\partial x}+Q-c_{b} W_{b}\left(T-T_{a r t}\right)$

The heat flux $q_{x}$ equals to $-k \frac{\partial T}{\partial x}$; hence,

$$
\begin{array}{r}
\rho c \frac{\partial T}{\partial t}=-\frac{\partial}{\partial x}\left(-k \frac{\partial T}{\partial x}\right)+Q-c_{b} W_{b}\left(T-T_{a r t}\right) \\
\rho c \frac{\partial T}{\partial t}=\frac{\partial}{\partial x}\left(k \frac{\partial T}{\partial x}\right)-c_{b} W_{b}\left(T-T_{a r t}\right)+Q
\end{array}
$$

Equation number (6.12) is solved in COMSOL with respect to certain boundary conditions. All of the thermal properties of the livers are constant and remain constant as the temperature changes throughout the materiel domain. In contrast, the thermal properties of the egg white are temperature-dependent up to $85^{\circ} \mathrm{C}$ and then they remain constant. The heat transfer in solids is considered while designing the fiber and the grating domains to restrict the effect of the blood perfusion to the material domain. The initial condition in the simulation is based on considering that the temperature of the material domain including the outer surface is equal to (1) the room temperature in the 
case of the egg white; and (2) the body core temperature in the case of the human and porcine liver.

$$
\begin{aligned}
& T(x, y, z, t=0)=T_{0} \\
& T(x s, y s, z s, t)=T_{s}=T_{0}
\end{aligned}
$$

where $T_{0}$ is the initial temperature, which was specified as $20{ }^{\circ} \mathrm{C}$ for the egg white and 37 ${ }^{\circ} \mathrm{C}$ for the livers.

These equations are solved in COMSOL using the finite element method [256-258, 269, $277,306]$. The approximate solution of equation (6.12) with respect to its boundary conditions is built into the solution process by first dividing the geometry into domains and then into smaller elements and nodes knows as mesh. The value of the variable at each element sharing a node has to be equal. These nodes have specific numbers to each element over which the interpolation functions are solved. The characteristics of individual elements are then defined by a matrix equation. Different techniques can be used to define the matrix equation including direct approach or iterative approach. After building the matrix equation, the software produces and solves the set of linear or nonlinear equations.

Besides the flexibility of the finite element method in solving complex geometric designs, it allows for the finding of various important parameters from the approximate solution of the system equations which is called post processing. For example, the system enables finding the values of heat flux and the degree of tissue damage in the bioheat transfer problem, in which the temperature field is the primary unknown variable. 


\section{3 Heat Source:}

Usually, the absorption power factor is used to indicate how much of the energy applied in a specific zone gets absorbed by tissues $[49,122-124,128]$. In our work, we want to specify the heat source term to be active in the active heater domain which generates constant thermal energy in the material domain. According to our active heater, we can drive the expression to the volumetric heat source $\mathrm{Q}$ by considering a light with intensity I propagating in the coating layer with the absorbance factor of medium $\alpha$ as:

$$
I=I_{0} e^{(-\alpha z)}
$$

where $I$ describes the amount of energy transferred per unit area ${ }^{p} /{ }_{A}\left(W^{-2}\right)$. To convert $I$ to the volumetric heat source $\mathrm{Q}\left(\mathrm{Wm}^{-3}\right)$, we assume that the light propagates from $z$ to $z+\Delta z$, with intensities $I_{z}$ and $I_{z+\Delta z}$. Between these two points the amount of the generated energy $\mathrm{Q}$ within a small distance is equal to:

$$
Q A \Delta z=\left(I_{z}-I_{z+\Delta z}\right) A
$$

where $\mathrm{A}$ is an area perpendicular to the direction of the propagating energy z. Dividing by $A \Delta z$ :

$$
\begin{gathered}
Q=\frac{\left(I_{z}-I_{z+\Delta z}\right)}{\Delta z} \\
Q=-\frac{\left(I_{z+\Delta z}-I_{z}\right)}{\Delta z}
\end{gathered}
$$

letting $\Delta z$ go to zero and using $\lim _{\Delta z \rightarrow 0} \frac{f(z+\Delta z)-f(z)}{\Delta z}=\frac{\partial f}{\partial z}$

$$
Q=-\frac{\partial I}{\partial z}
$$

where $I=I_{0} e^{(-\alpha z)}$ 


$$
\begin{aligned}
& Q=-\frac{\partial}{\partial z}\left(I_{0} e^{(-\alpha z)}\right) \\
& Q=I_{0} \alpha e^{(-\alpha z)}
\end{aligned}
$$

where $\mathrm{z}$ is the grating length $(10 \mathrm{~mm})$, and $\alpha=25\left(\frac{1}{\mathrm{~m}}\right)$ is the absorbance factor of the coated material. This value of the absorbance factor was selected to obtain the maximum possible absorption between the other different trail values since we assumed that all of the light is converted into heat.

Based on the experiment setup, we calculated $I_{0}$ from the applied pumping power as:

$$
I_{0}=p / \pi r^{2}
$$

where $r$ equals $0.0625 \mathrm{~mm}$ which is the radius of the cladding layer. $p$ is the experimentally applied pumping power.

In the experiment setup, we used a coupler which introduces a $10 \%$ loss to the total pumping powers in order of $1 \mathrm{~W}, 1.2 \mathrm{~W}$ and $2 \mathrm{~W}$. Also $84 \%$ of the pump power is sent to the cladding and the coating layer. Thus, a pumping power in order of $0.756 \mathrm{~W}, 0.9072$, and $1.512 \mathrm{~W}$ is used to induce heat in the coating layer. Hence, the maximum initial intensities utilized in the simulation were in order of $61.6 \mathrm{~W} / \mathrm{mm}^{2}, 73.96 \mathrm{~W} / \mathrm{mm}^{2}$, $123.27 \mathrm{~W} / \mathrm{mm}^{2}$

\section{4 Input Parameters:}

Ascertaining the extent of thermal damage and its degree in tissue is required from the heat transfer models of hyperthermia [259-275]. Accurate estimation of these thermal properties is imperative to determine the heat transfer and its effect on a biological system. The amount of the thermal energy increase in the materials domain depends on

their thermal properties, density, thermal conductivity, and thermal capacity. Thus, in our 
model we were very careful in estimating the materials' properties and in considering the experimental setup and data, mainly performed in the egg white phantom.

COMSOL software provides two methods, temperature threshold and energy absorption, to measure the degree of damage caused by the thermal energy in tissue. In our models, we used the temperature threshold to determine the degree of damage caused by the active heater. Determining the degree of heat-induced tissue damage in this method is based on the time period of exposing the tissue to a critical temperature. Upon the experiment data of the heat-induced protein denaturation of the egg white, we define the damage temperature and time as $55^{\circ} \mathrm{C}$ for 13.8 seconds.

\section{4. 1 Egg White:}

Heat is a key parameter which triggers many protein aggregation processes [219-247]. Coagulation accompanied by changes in configuration is the aggregation process of protein in which its presence in the native state is usually dissolved in water. This biological phenomenon, called protein denaturation, is very important in understanding various biological activities and structures such as blood clots.

The denaturation of the egg white protein involves changing from the native to the denatured phase of the protein [219-247]. This includes the breakage of hydrogen bonds, the uncoiling of polypeptide chain, the exposure of reactive groups, and the formation of a stable intermolecular $\beta$-sheet. These conformational changes directly influence the egg white's thermal properties. Thermal analysis of the denatured temperatures of each protein in the egg white has been vastly explored in view of the fact that different and wide usages of the egg white protein can be exemplified as a model to understand the 
formation of specific structure such as fibrils and as an antimicrobial edible film for food packaging [246].

Figure 6.4. shows the schematic diagram of the effect of heat on the egg white [230]. The protein's denaturation usually materializes visually by a fast transition process starting with the transformation of the egg white's transparent liquid into a soft white lesion and then a solid lesion [219-247]. The denaturation temperature range of the egg white protein is identified to be between 55 and $85^{\circ} \mathrm{C}$. The lowest denatured temperature is reportedly between 55 and $61^{\circ} \mathrm{C}$, depending on the $\mathrm{pH}$ of the egg white and the energy source $[231,232,239]$.

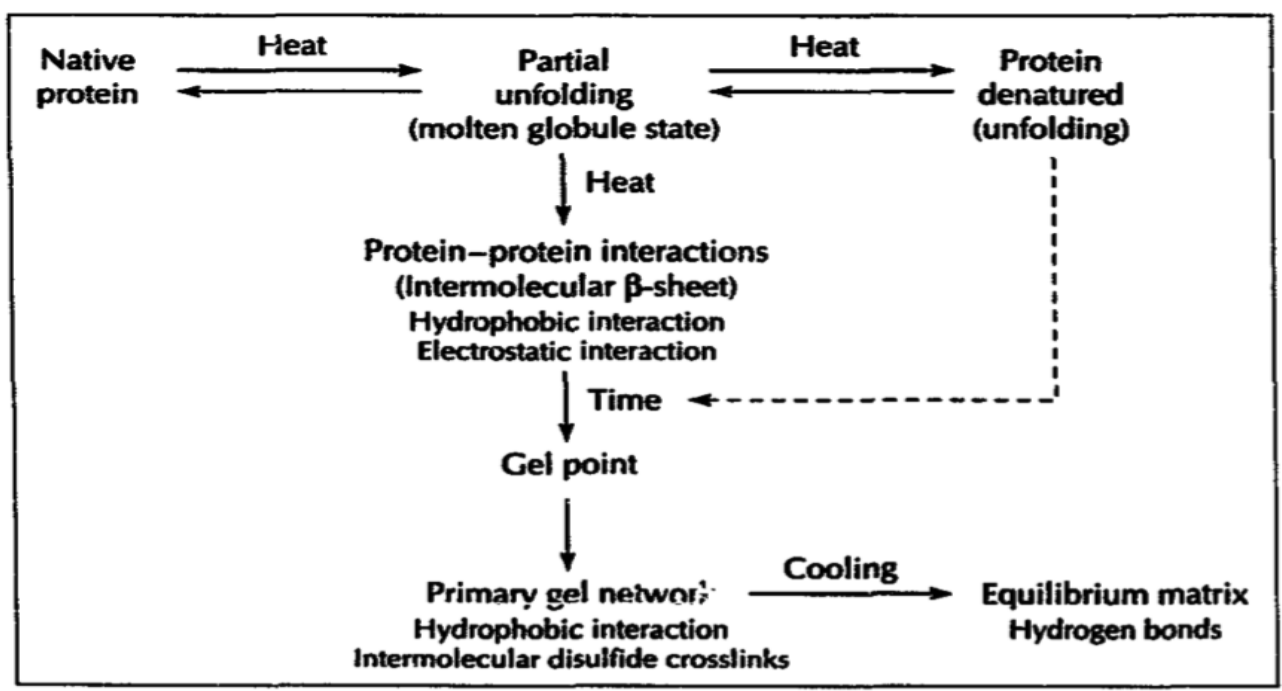

Figure 6.4. Schematic diagram of the egg white denaturation process [230].

Based on the experiment results, we consider $55^{\circ} \mathrm{C}$ to be the transitional denatured temperature. Beyond $85^{\circ} \mathrm{C}$, the egg white's properties are considered constant and not affected by temperature changes. This effect of the coagulation process on the egg white's thermal properties is considered in the simulation through using the following equations [224, 225, 241, 242]:

For the egg white density: 


$$
\rho=\left[\begin{array}{cc}
\sum x_{i} \rho_{i} & 20^{\circ} \mathrm{C} \leq T \leq 85^{\circ} \mathrm{C} \\
1013.8 & T>85^{\circ} \mathrm{C}
\end{array}\right.
$$

where $\rho_{i}$ is the density of each of the egg white components:

$$
\begin{aligned}
& \rho_{\text {water }}=9.9718 \times 10^{2}+3.1439 \times 10^{-3} \mathrm{~T}-3.7574 \times 10^{-3} \mathrm{~T}^{2} \\
& \rho_{\text {protein }}=1.3299 \times 10^{3}-5.1840 \times 10^{-1} \mathrm{~T} \\
& \rho_{\text {fat }}=9.2559 \times 10^{2}-4.1757 \times 10^{-1} \mathrm{~T} \\
& \rho_{\text {carbohydrate }}=1.5991 \times 10^{3}-3.1046 \times 10^{-1} \mathrm{~T} \\
& \rho_{\text {ash }}=2.4238 \times 10^{3}-2.8063 \times 10^{-1} \mathrm{~T}
\end{aligned}
$$

Also $x_{i}$ is the percentage of each of the egg white components:

$$
\begin{aligned}
& x_{\text {water }}=87.3 \% \\
& x_{\text {protein }}=10.25 \% \\
& x_{\text {fat }}=0.05 \% \\
& x_{\text {carbohydrate }}=0.975 \% \\
& x_{\text {ash }}=0.055 \%
\end{aligned}
$$

For the egg white specific heat:

$$
c_{p}=\left[\begin{array}{cc}
\sum x_{i} c_{p i} & 20^{\circ} \mathrm{C} \leq T \leq 85^{\circ} \mathrm{C} \\
3865.2 & T>85^{\circ} \mathrm{C}
\end{array}\right.
$$

where $c_{p i}$ is the specific heat of each of the egg white components:

$$
\begin{aligned}
& c_{\text {pwater }_{(-40 \text { to } 0)^{\circ} \mathrm{C}}}=4.1289-5.3062 \times 10^{-3} \mathrm{~T}+9.9516 \times 10^{-4} \mathrm{~T}^{2} \\
& c_{\text {pwater }_{(0 \text { to } 150)^{\circ} \mathrm{C}}}=4.1289-9.0864 \times 10^{-5} \mathrm{~T}+5.4731 \times 10^{-6} \mathrm{~T}^{2} \\
& c_{\text {pprotein }}=2.0082+1.2089 \times 10^{-3} \mathrm{~T}-1.3129 \times 10^{-6} \mathrm{~T}^{2} \\
& c_{\text {pfat }}=1.9842+1.4733 \times 10^{-3} \mathrm{~T}-4.8008 \times 10^{-6} \mathrm{~T}^{2} \\
& c_{\text {pcarbohydrate }}=1.5488+1.9625 \times 10^{-3} \mathrm{~T}-5.9399 \times 10^{-6} \mathrm{~T}^{2} \\
& c_{\text {pash }}=1.0926+1.8896 \times 10^{-3} \mathrm{~T}-3.6817 \times 10^{-6} \mathrm{~T}^{2}
\end{aligned}
$$

For the egg white thermal conductivity: 


$$
k=\left[\begin{array}{cc}
\sum \frac{1}{\left(e_{i} / k_{i}\right)} & 20^{\circ} \mathrm{C} \leq T \leq 85^{\circ} \mathrm{C} \\
0.5752 & T>85^{\circ} \mathrm{C}
\end{array}\right.
$$

$e_{i}$ equals to:

$$
\begin{aligned}
& e_{\text {water }}=\left(1042.3 \times x_{\text {water }}\right) / \rho_{\text {water }} \\
& e_{\text {protein }}=\left(1042.3 \times x_{\text {protein }}\right) / \rho_{\text {protein }} \\
& e_{\text {fat }}=\left(1042.3 \times x_{\text {fat }}\right) / \rho_{\text {fat }} \\
& e_{\text {carbohydrate }}=\left(1042.3 \times x_{\text {carbohydrate }}\right) / \rho_{\text {carbohydrate }} \\
& e_{\text {ash }}=\left(1042.3 \times x_{\text {ash }}\right) / \rho_{\text {ash }}
\end{aligned}
$$

where $k_{i}$ is the thermal conductivity of each of the egg white components:

$$
\begin{gathered}
k_{\text {water }}=5.7109 \times 10^{-1}+1.7625 \times 10^{-3} \mathrm{~T}-6.7036 \times 10^{-6} \mathrm{~T}^{2} \\
k_{\text {protein }}=1.7881 \times 10^{-1}+1.1958 \times 10^{-3} \mathrm{~T}-2.7178 \times 10^{-6} \mathrm{~T}^{2} \\
k_{\text {fat }}=1.8071 \times 10^{-1}-2.7604 \times 10^{-4} \mathrm{~T}-1.7749 \times 10^{-7} \mathrm{~T}^{2} \\
k_{\text {carbohydrate }}=2.0141 \times 10^{-1}+1.3874 \times 10^{-3} \mathrm{~T}-4.3312 \times 10^{-6} \mathrm{~T}^{2} \\
k_{\text {ash }}=3.2962 \times 10^{-1}+1.4011 \times 10^{-3} \mathrm{~T}-2.9069 \times 10^{-6} \mathrm{~T}^{2}
\end{gathered}
$$

The curves in Figure 6.5. demonstrate the rate of change in the egg white's thermal properties as a function of temperature ranging in Figures $(6.6,6.7$, and 6.8)) or [221, $224,230-236,238]$. 

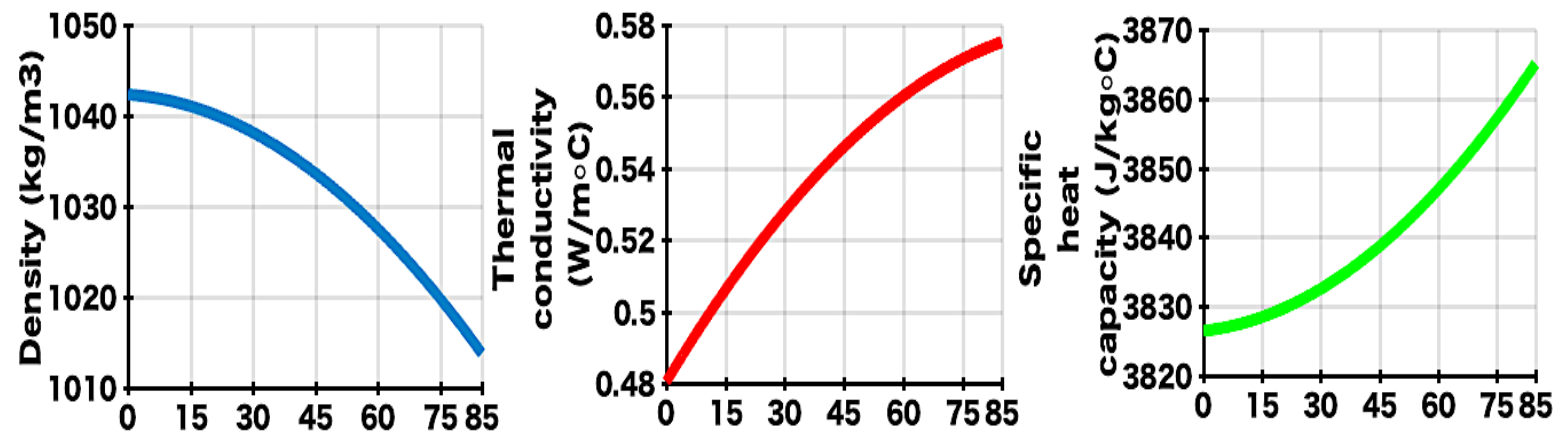

Temperature (०C)

Figure 6.5. Egg white properties as a function of Temperature.

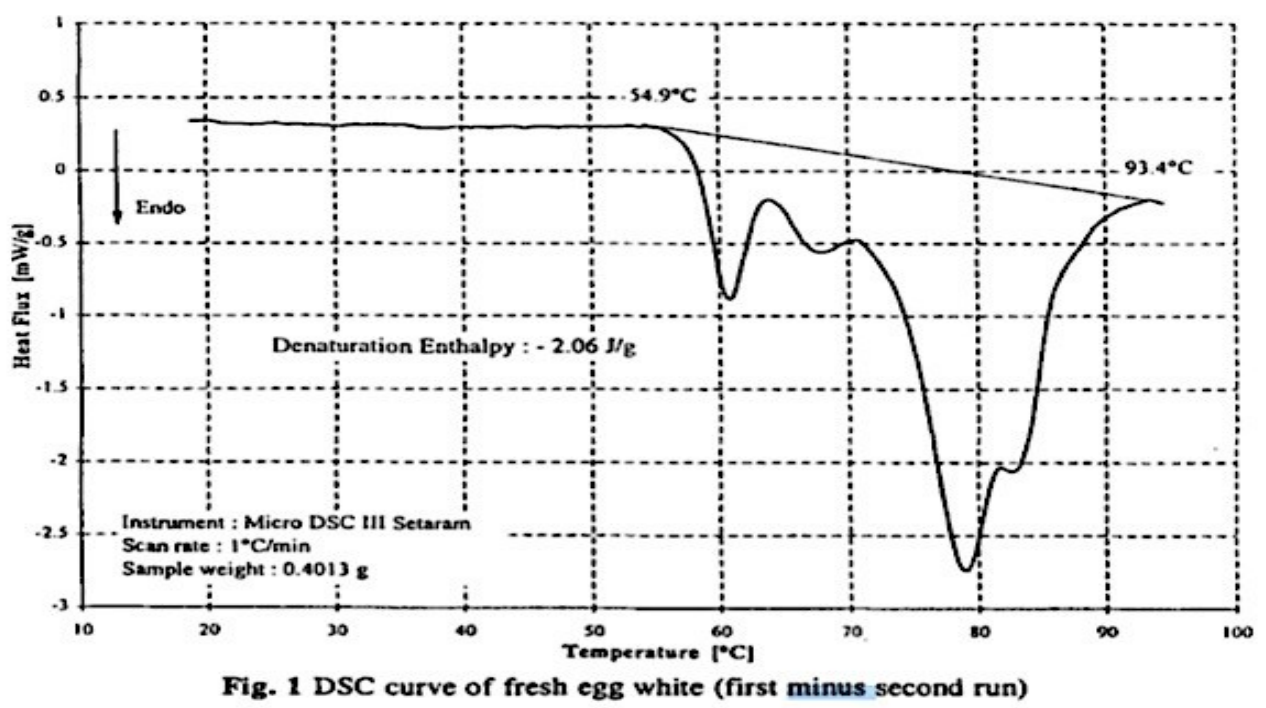

Figure 6.6. Heat flux of the egg white as a function of temperature [226]. 


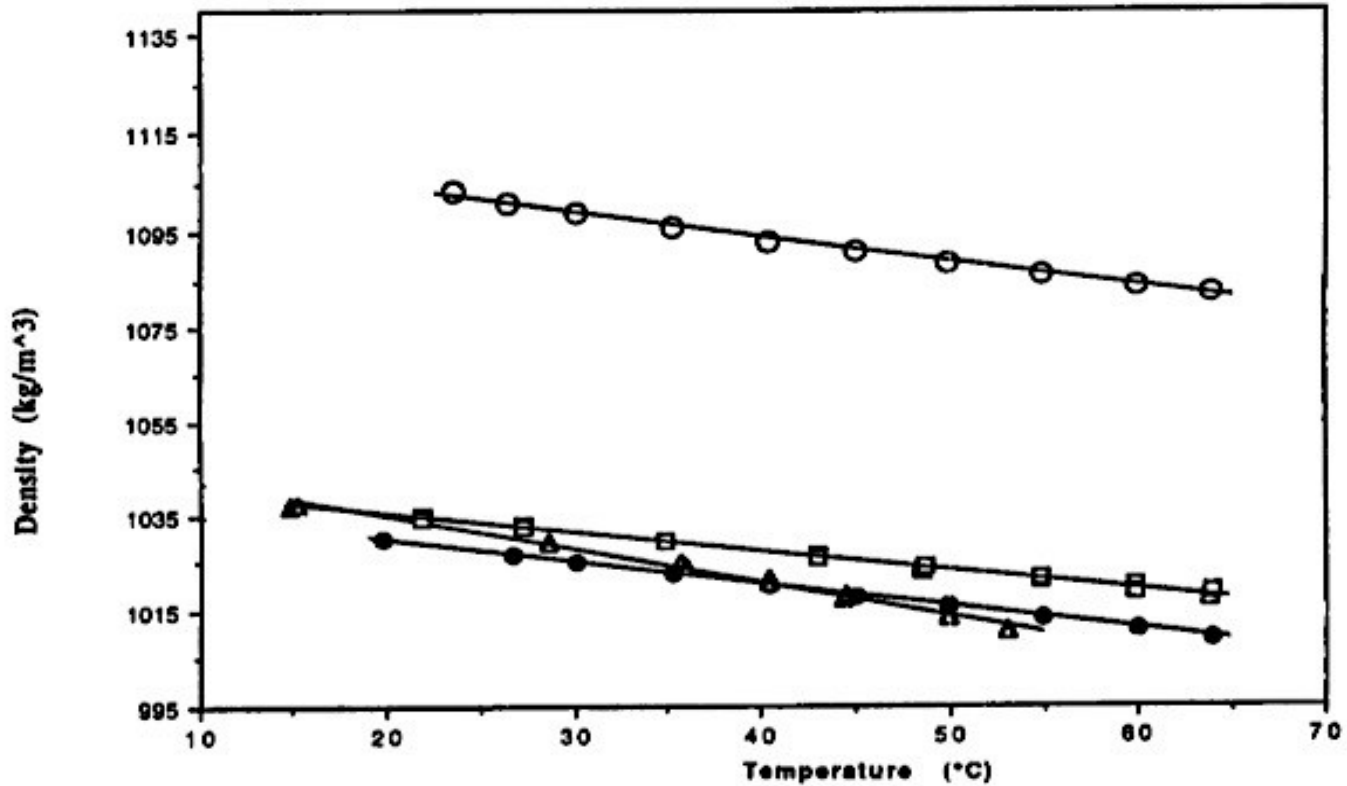

FIG. 1. DENSITY OF DIFFERENT EGG PRODUCTS AS A FUNCTION OF TEMPERATURE (-O-Egg yolk-salted, - $\Delta$ - egg white,- $\square$-whole egg, - - egg yolk)

Figure 6.7 Density of the egg white as a function of temperature [236].

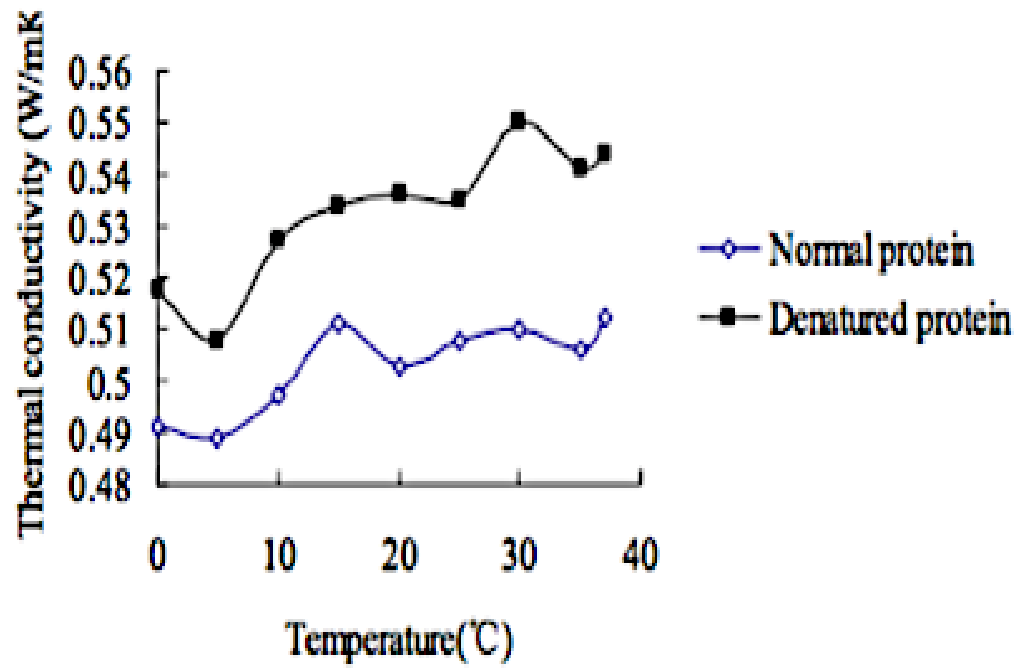

Figure 6.8. Thermal conductivity of a protein as function of temperature [260]. 
For the other domains' properties, we used the silica and carbon dioxide thermal properties to simulate the thermal response in the fiber and the gratings areas [275]. Table 6.3. shows the thermal properties of the fibers and the gratings' domains. Thermal properties of the porcine/human liver and the blood are also shown in Table 6.3. [264, 273].

\begin{tabular}{|c|c|c|c|}
\hline \multirow{2}{*}{ Symbols } & \multicolumn{3}{|c|}{ Definition } \\
\hline & Name & Value & Unit \\
\hline \multirow{5}{*}{$\rho$} & Human liver density & 1079 & \multirow{5}{*}{$\mathrm{Kgm}^{-3}$} \\
\hline & Porcine liver density & 1110 & \\
\hline & Blood density & 1000 & \\
\hline & Amorphous carbon density & 2356 & \\
\hline & Silica density & 2203 & \\
\hline \multirow{5}{*}{$c_{p}$} & Human liver specific heat & 3540 & \multirow{5}{*}{$J K^{-1} K^{-1}$} \\
\hline & Porcine liver specific heat & 3800 & \\
\hline & Blood specific heat & 4180 & \\
\hline & Amorphous carbon specific heat & 800 & \\
\hline & Silica specific heat & 703 & \\
\hline \multirow{4}{*}{$k$} & Human liver thermal conductivity & 0.52 & \multirow{4}{*}{$W m^{-1} K^{-1}$} \\
\hline & Porcine liver thermal conductivity & 0.56 & \\
\hline & $\begin{array}{l}\text { Amorphous carbon thermal } \\
\text { conductivity }\end{array}$ & 10 & \\
\hline & Silica thermal conductivity & 1.38 & \\
\hline$W_{b}$ & Blood perfusion rate & $6.4 e^{-3}$ & $1 / \mathrm{s}$ \\
\hline
\end{tabular}

Table 6.3. Thermal properties of the utilized materials in the simulation. 


\section{Chapter 7: Results of Mathematical Modelling}

In this chapter we show good agreement between the experimental and computational results by comparing two points: (1) the values of the temperature increase at different locations away from the TFBG-based active heater when pumping at different powers; and (2) the dimension of the damaged volumes obtained from pumping at different powers; After we validate our simulation, we illustrate the active heater performance in real environments by considering the blood perfusion effect in liver tissues.

\section{1 Computational Results of Hyperthermia in Egg White Phantom (model validation):}

Besides the similarity in thermal properties of the egg white to those of human tissues (figure 4.8), the transparency of the egg white allows the visualization of the depth, degree, and shape of the thermal damage caused by localized hyperthermia. Thus, we use the egg white phantom in our study to easily visualize the compression of the whole 3D treatment zone geometry between the experimental and computational studies. Ascertaining the extent of thermal damage and its degree in tissue is required from the heat transfer models of hyperthermia. Accurate estimation of these thermal properties is imperative to determine the heat transfer and its effect on a biological system. Thus, we considered the coagulation process of the egg white's thermal properties in the simulation as described in Chapter 6.

Figures 7.1 and 7.2 show the experimental and calculated effect of heating at two pump powers of $1 \mathrm{~W}$ and $1.2 \mathrm{~W}$ for 5 minutes in egg white. As we mentioned before that we use the experimental setup and observation as a base for the simulation input data, thereby, we consider the egg white protein denaturation to occur when the formation of a thin layer of a transparent white lesion starts. This was observed when the temperature 


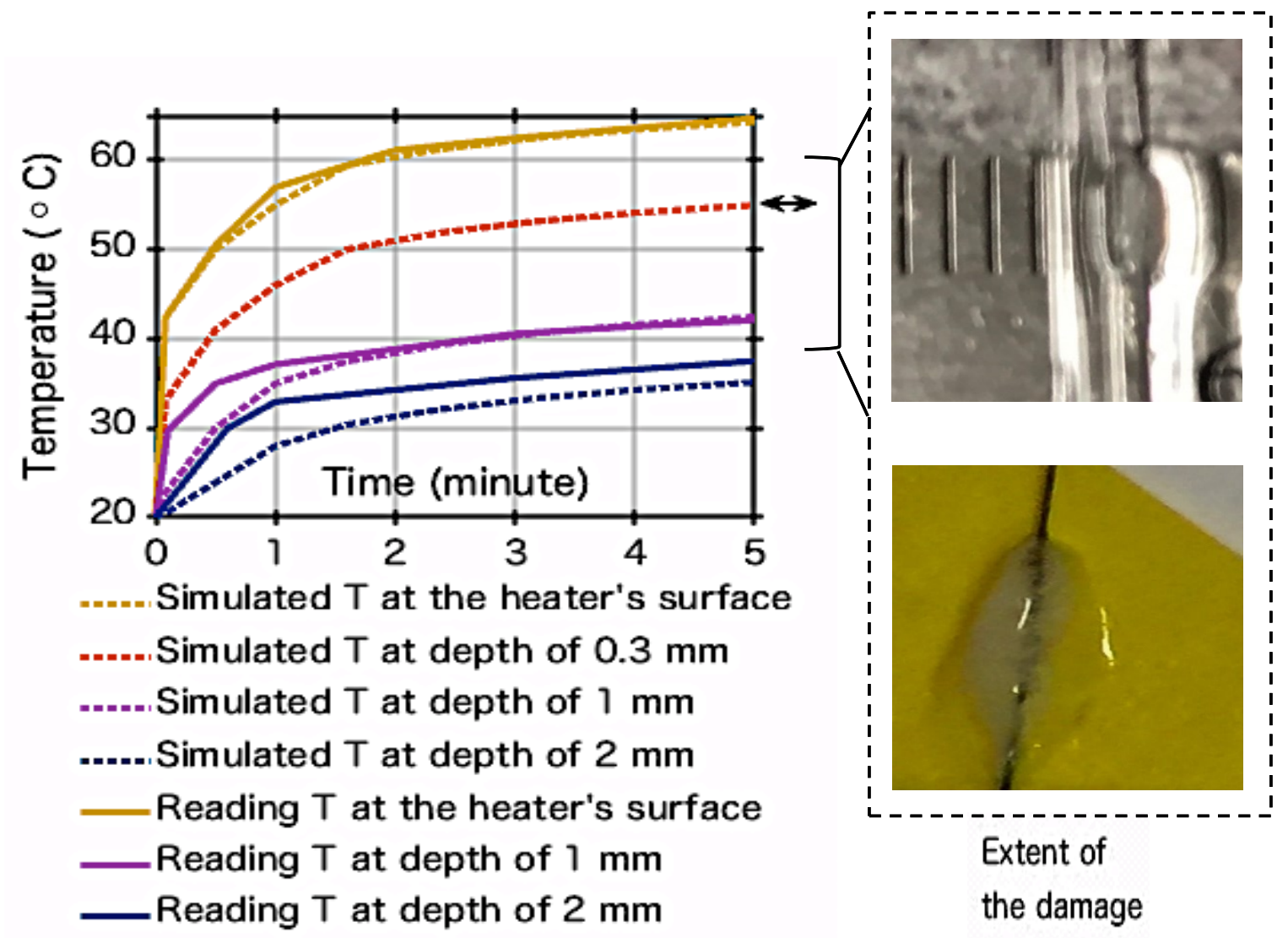

Figure 7.1. The experimental and calculated effect of heating at pump power of $1 \mathrm{~W}$ for 5 minutes in egg white: (a) comparison of the temperature variations at different locations between the simulation (dotted curves) and the experiment (solid curves) results.

reading at the surface of the active heater stated a temperature increase of an approximate $53 \pm 2{ }^{\circ} \mathrm{C}$ at 1.2 minutes and at 0.43 minutes after starting the heating process at $1 \mathrm{~W}$ and $1.2 \mathrm{~W}$ respectively. A temperature increases of $44.303{ }^{\circ} \mathrm{C}$ and $60.19{ }^{\circ} \mathrm{C}$ at the active heater caused a lesion with radius of less than $0.5 \mathrm{~mm}$ and $0.7 \mathrm{~mm}$ experimentally (Figure $7.1 \&$ Figure 7.2) and with calculated radius of $0.34 \mathrm{~mm}$ and $0.7 \mathrm{~mm}$ (Figure 7.1 \& Figure 7.2) when pumping at $1 \mathrm{~W}$ and $1.2 \mathrm{~W}$ respectively. At pump power of $1 \mathrm{~W}$, we could not measure the value of the elevated temperature at $0.3 \mathrm{~mm}$ because the closest FBGs probe to the active heater was at a distance of $1 \mathrm{~mm}$. However, other temperature values at $1 \mathrm{~mm}$ and $2 \mathrm{~mm}$ match well with the simulated values. 

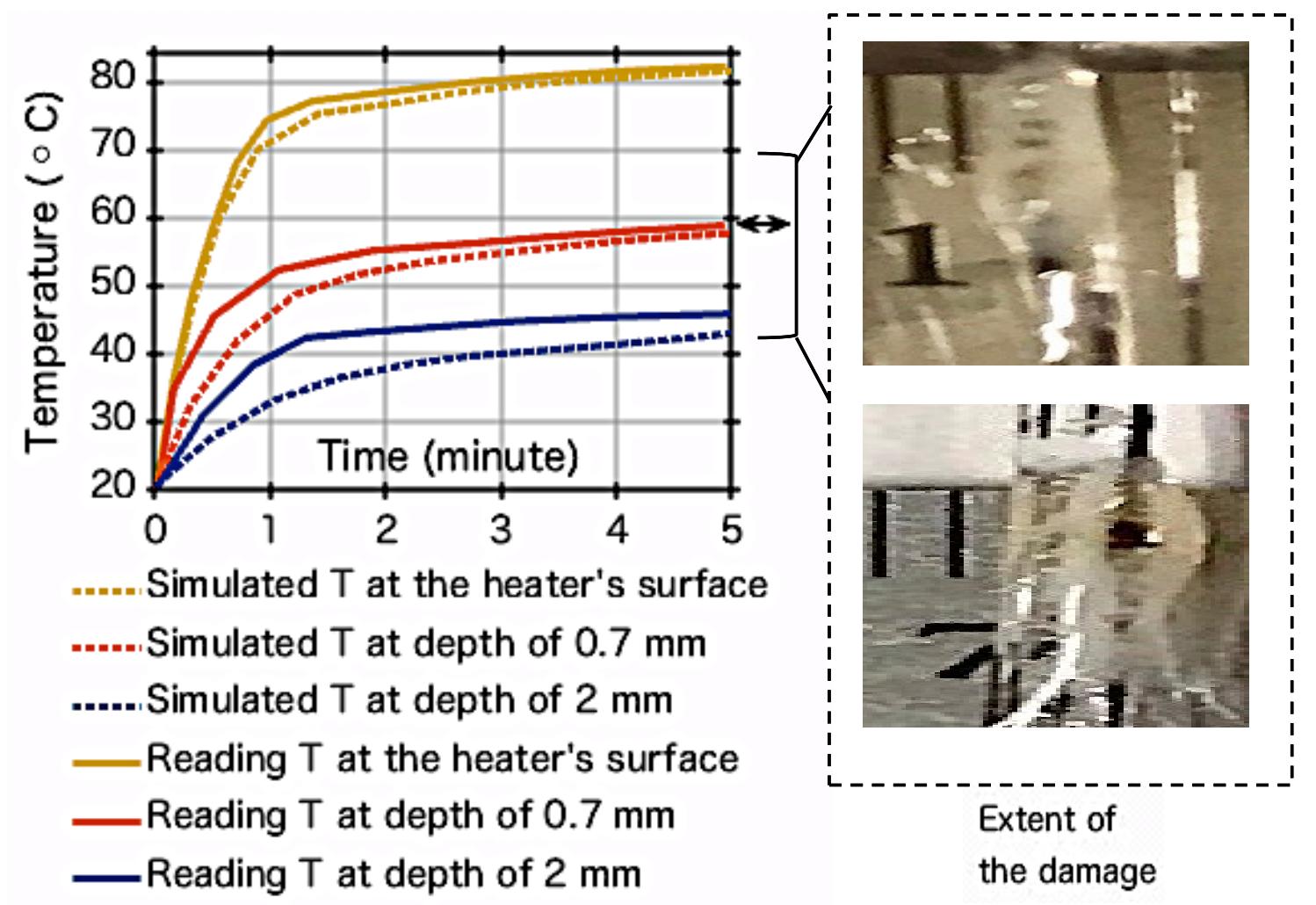

Figure 7.2. The experimental and calculated effect of heating at pump power of $1.2 \mathrm{~W}$ for 5 minutes in egg white: (a) comparison of the temperature variations at different locations between the simulation (dotted curves) and the experiment (solid curves) results.

Figures 7.3 and 7.4. show the dimensions of the lethal damage in the hen egg white and porcine liver without the blood perfusion effect. Irreversible thermal damage of tissue usually occurs in therapeutic temperatures ranging from $55^{\circ} \mathrm{C}$ to $95^{\circ} \mathrm{C}[20,89,95,224$, $240,241,259]$. Based upon that we define the damage temperature as $55^{\circ} \mathrm{C}$ for the porcine liver tissues. Thus, similar lesion dimensions are obtained in hen egg white and porcine liver, with lesions of approximately $5 \times 12 \mathrm{~mm}$ and $7 \times 15 \mathrm{~mm}$ experimentally and with calculated lesions of approximately $4.87 \times 11.6 \mathrm{~mm}$ and $8.8 \times 14.3 \mathrm{~mm}$ in both tissues respectively. 
a)

b)
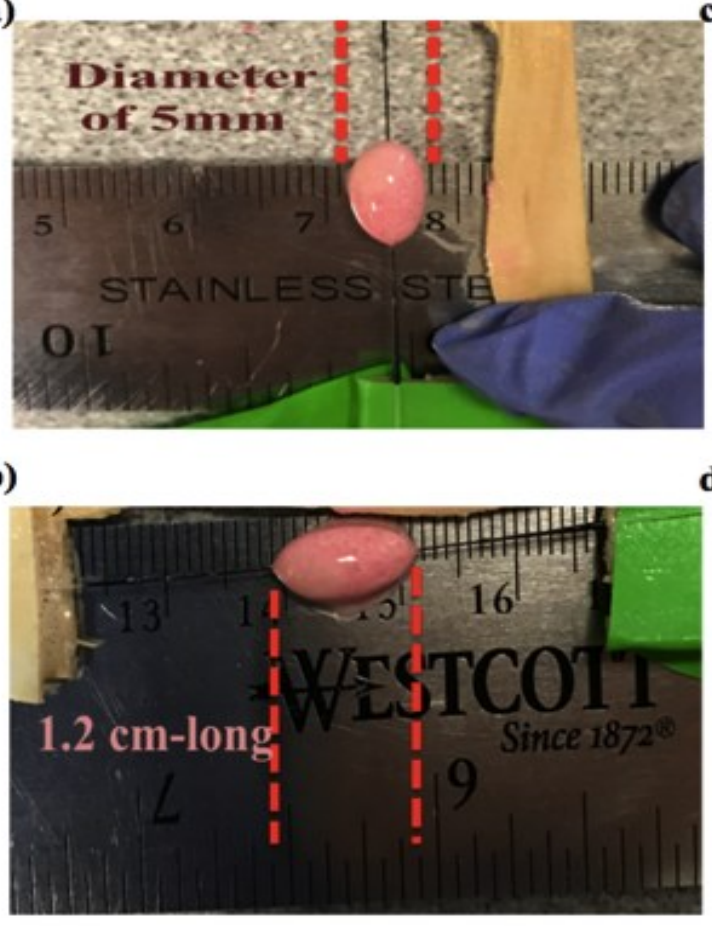

c)

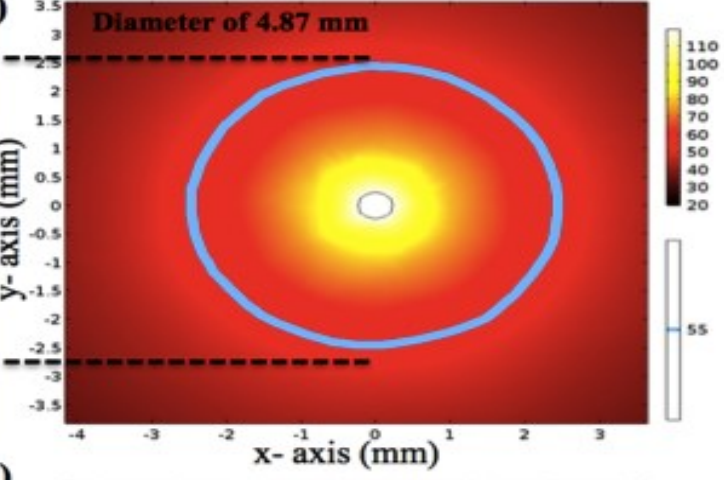

d)

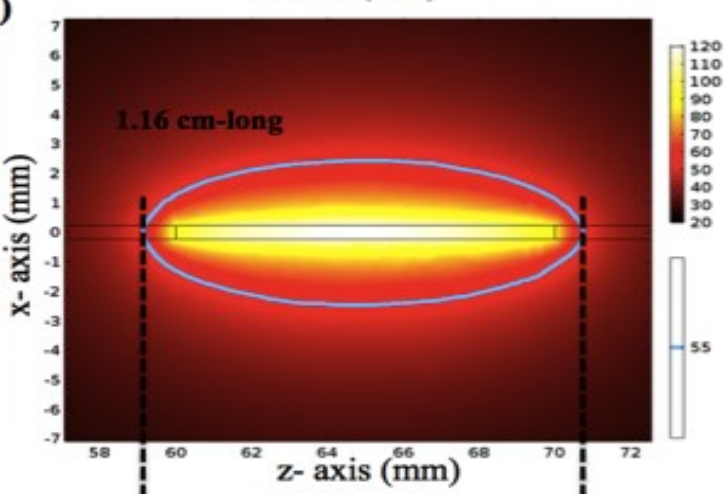

Figure 7.3. The experimental (a \&b) and calculated (c \&d) effect of heating at pump power of $1.8 \mathrm{~W}$ for 10 minutes in egg white: the dimensions of the obtained lesion; based on the experiment results, we consider $55^{\circ} \mathrm{C}$ to be the transitional denatured temperature (represented by the blue circle and ellipse in c \& d). 

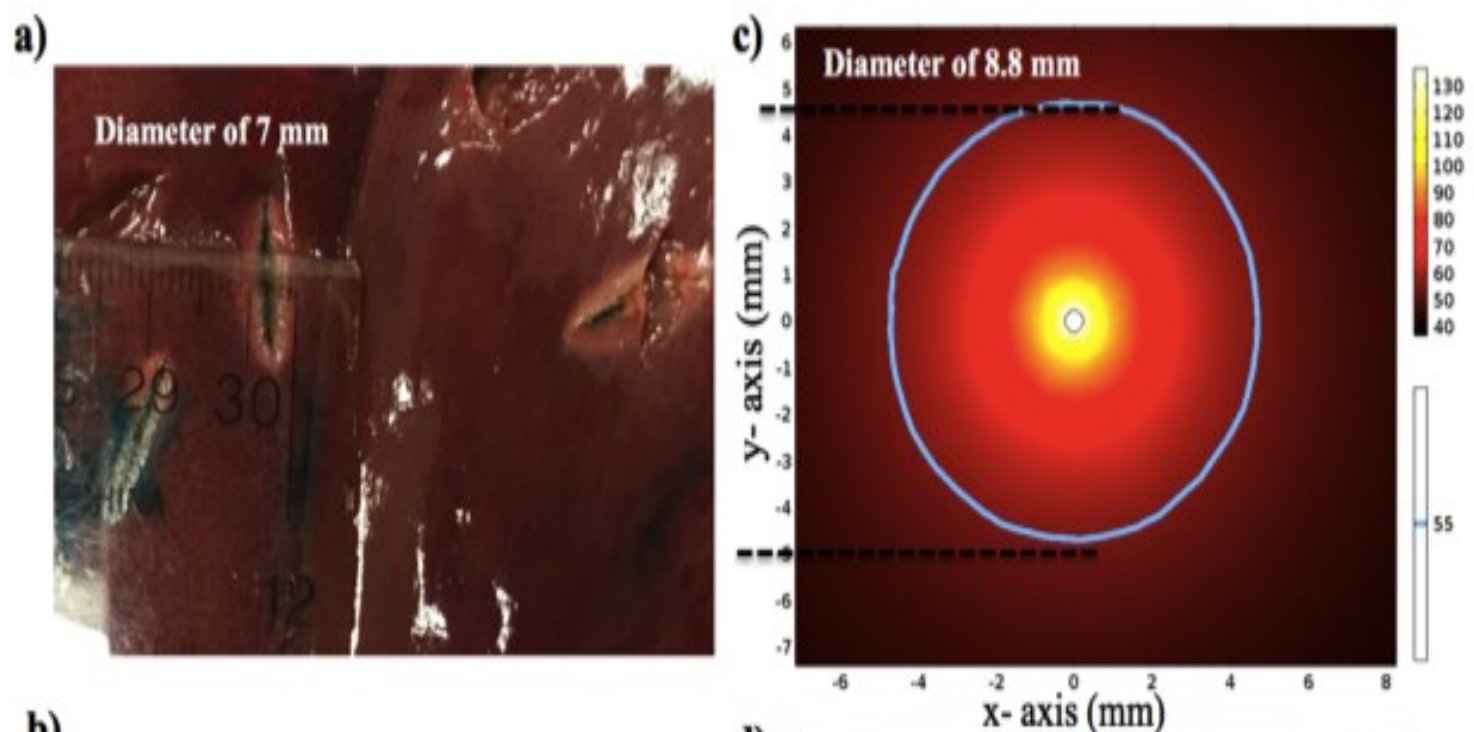

b)

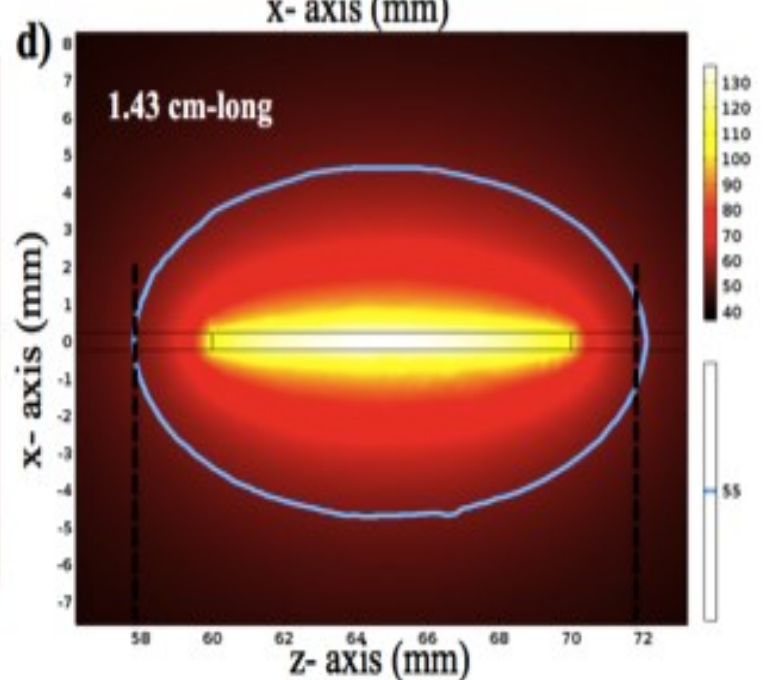

Figure 7.4. The experimental (a \&b) and calculated (c \&d) effect of heating at pump power of $1.8 \mathrm{~W}$ for 10 minutes in porcine liver.

In the heat transfer model, temperature increase reaches a steady state 90 seconds after turning on the heating power. Figure 7.5. shows the temperature increase along the gratings' surface as a function of time. After 5 minutes, the increase in temperature becomes very small with a total additional increase of $4{ }^{\circ} \mathrm{C}$ at 5 minutes and $7{ }^{\circ} \mathrm{C}$ at 10 minutes. Thus, the extent of hyperthermia should be determined within the first 5 minutes of starting the heating process. Any further heating will affect the degree of the thermal damage in the confined volume as can be seen in Figure 5.9. 


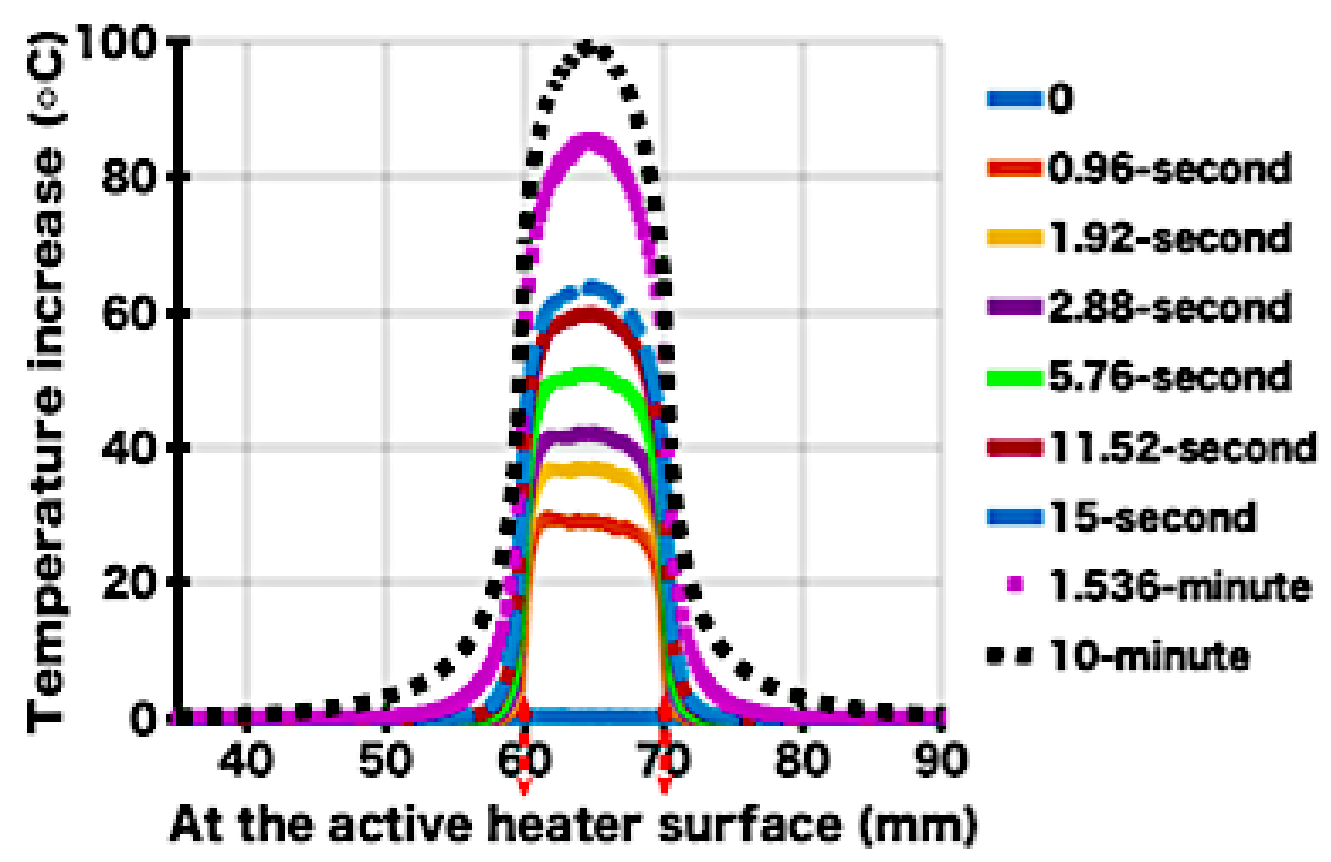

Figure 7.5. Temperature increases along the gratings' surface as a function of time.

\subsection{Computational Results of Hyperthermia in Liver Tissues:}

After validating this heat transfer model with experimental results, we simulate the effect of blood perfusion on the heat-induced damage caused by the active heater in porcine and human livers. Figure 7.6 shows the temperature field at the grating surface and at different distances from the active heater. Heating at $1.8 \mathrm{~W}$ for 10 minutes causes a temperature increase of 121.96 and $127.54{ }^{\circ} \mathrm{C}$ at the active heater surface in porcine liver and human liver, respectively. Under the same heating condition in the porcine and human livers without considering the effect of the blood perfusion on the induced heat, the temperature increase at the active heater surface is 135.93 and $143.46^{\circ} \mathrm{C}$ for both tissues respectively. Thus, the significant heat-induced loss with a reduction in order of $10.27 \%$ and $11.09 \%$ is calculated at the heat source for both tissues respectively. On the other hand, the effect of the blood perfusion on the temperature gradient increase caused 
a)

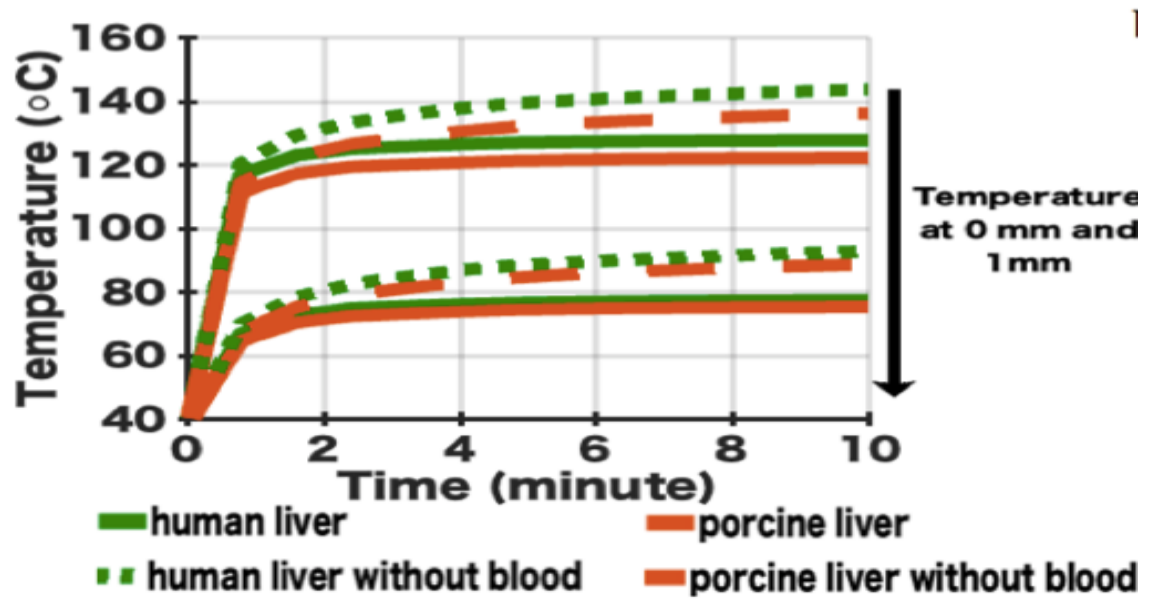

b)

\begin{tabular}{|c|c|c|c|c|}
\hline Temperature ${ }^{\circ} \mathrm{C}$ & $\begin{array}{l}\text { At the } \\
\text { active } \\
\text { heater }\end{array}$ & $1 \mathrm{~mm}$ & $2 \mathrm{~mm}$ & $3 \mathrm{~mm}$ \\
\hline Human liver & 127.54 & 77.15 & 61.89 & 53.48 \\
\hline $\begin{array}{l}\text { Human liver } \\
\text { without blood } \\
\text { perfusion }\end{array}$ & 143.46 & 92.42 & 75.6 & 65.5 \\
\hline Porcine liver & 121.96 & 75.03 & 60.75 & 52.84 \\
\hline $\begin{array}{l}\text { Porcine liver } \\
\text { without blood } \\
\text { perfusion }\end{array}$ & 135.93 & $\mathbf{8 8 . 4 3}$ & 72.85 & 63.46 \\
\hline
\end{tabular}

Figure 7.6. Simulate the effect of blood perfusion on the heat-induced damage caused by the active heater in porcine and human livers.

by the flow of heat from the active heater surface into the heated target was very small in the order of $3.57 \%$ and $3.93 \%$ at a distance of $4 \mathrm{~mm}$ from the heat source for both tissues respectively. 


\section{Chapter 8: Conclusion}

Cancer is a class of diseases which are reportedly the most challenging and life-threating as each healthy cell in the human body can be a tumour's seed. At this point there is no completely effective therapy for cancer; hence, a combination of cancer treatments techniques is usually utilized to treat a malignant growth $[1-3,15,17]$. Targeted treatment for certain types of tumours and being less harmful overall to the body comprise the attractive features of localized hyperthermia therapy. For this thermal therapy technique that is used to treat different sizes, types, and stages of tumours, scientists are working on improving the current heating system and developing new techniques. For example, the radiofrequency ablation technique has been designed to treat isolated small tumours less than $5 \mathrm{~cm}$ in diameter, while the microwave localized hyperthermia has been developed to potentially cause deep destruction to the targeted tumours with size of an approximate of $10 \mathrm{~cm}$ in diameter.

In this thesis work, the thermal damage in the tumour tissues is caused by the thermal conduction from the active heater surface into the target material. The single heater produces a lethal volume with length and diameter in order of $7 \times 15 \mathrm{~mm}$. This active heater provides the following advantages over the other localized thermal therapy techniques:

1) The temperature increase within the tumour tissue does not depend on the amount of ions in the tissue, as in the case of radiofrequency ablation; or on the amount of water in the tissue, for example in microwave localized hyperthermia. The only heat source within the material is heat conduction, which depends only on the material thermal properties; therefore, control lethal volume can be produced 
(Figure 5.5, when we carried out the experiments in living cells, we got a sharp boundary between the stained (dead) cells and the clear cells (living))

2) The small produced lesion provides a reliable real-time reading of the temperature increase. As indicated in the literature, the flow of heat effect can be estimated within a distance in order of $4 \mathrm{~mm}$ from the heating device simply by knowing the applicator's surface temperature.

3) The TFBGs' surface temperature can be controlled and determined with high accuracy and fast response time by observing the shift in Bragg wavelength. Thus, this active heater provides the additional advantage of eliminating the need for an additional external thermometer, which is a common requirement in localized thermal therapy.

In this thesis work, we show a good control of the heating profile of the TFBGs' active heater in three different cell lines — two healthy cells (HEK 293 and MCF-10A) and a cancer cell (MCF7) - and in tissue-like material. We also simulate the heat profile in liver tissue, which has been supported by the ex vivo studies in pig liver [323]. The calculated effect of blood circulation in the conductive heat profile of the TFBGs' active heater in living tissue is insignificant due to the small lethal volume.

\section{1 Future Works:}

\section{1. 1 Packaging the Device:}

Biocompatibility and sensor performance must be considered in the packaging design of this TFBGs-based active heater to be potentially used for medical procedures [44- 48]. Materials must be chosen carefully to avoid reduction in the heating efficiency and the thermal sensitivity of the device, in other words the response time of the device. 
Biocompatibility requires the device to be non-toxic, non-allergenic, non-irritating, nongenetic toxic, and non-carcinogenic $[44,45]$. Since this device will be in direct contact with human tissues under extreme conditions in addition to the non-predictable biochemistry reaction in the organism, more in vitro studies are necessary before applying the heater in vivo in animals.

\section{1.2 Design Multiple Localized Heating Points:}

For the TFBG-based active heater to be effectively used, enlargement of the heating zone is necessary. This enlargement can be achieved by writing a number of gratings into a single fiber. This would require multiple localized heating points with built-in thermometers. However, appropriate design must be developed in order for each heating zone to receive enough pump power, hence delivering efficient treatment. Fortunately, the feasible design of TFBGs will allow for this requirement to become obtainable in the near future. 


\section{Appendix}

\section{1. Alternative bioheat transfer models:}

In 1974, Wulff questioned the theoretical basis of Pennes' work and presented a modified version of the bioheat transfer equation by just considering the effect of directional blood perfusion on heat transfer. Chen and Holmes in 1980 developed a more advanced heat transfer concept with a physically and physiologically solid consistent therotical basis known as the $\mathrm{CH}$ model [279]. This model can provide a very accurate solution to heat transfer in living tissues. Their eqaution is similar to Pennes' bioheat transfer equation with the following modifications: (1) the blood perfusion term is specified for only the smallest blood vessels because including large blood vessels, such as $1 \mathrm{~mm}$ blood vessels, violates the continuum assumption in the bioheat transfer equation that "the dimension of the individual blood vessels is very small compared to the length scale of the changes in the tissue temperature"; (2) the term $\nabla \cdot\left(K_{t} \nabla T\right)$ is modified in the bioheat equation to $\nabla \cdot\left(k+k_{p}\right) \nabla T$ to consider the boost in heat transfer within the tissue due to blood perfusion conductivty $\left(k_{p}\right)$; and (3) the conductive heat transfer term $\left(-c_{b} \rho_{b} u\right.$. $\nabla T)$ is added to the equation to describe the thermal interaction between blood vessels and tissues.

Later in 1984, Weinbaum, Jiji, and Lemons added a significant contribution to the bioheat transfer field through a series of studies which resulted in two models: WJ and WJL [280-283]. The authors conducted various studies on the vascular network of rabbit thighs. Their conclusion states that the primary cause of heat transfer in tissues is the blood vessels in countercurrent pairs. Based on blood perfusion of healthy tissues, they formulated their mathematical model. Thus, some modifications have to be made on their 
mathematical model in order to be able to describe the effect of blood perfusion on heat transfer in tumors.

These are some of the mathematical models which have been developed to describe heat transfer within biological systems. The blood perfusion is the focal factor in all of these models, since blood perfusion is the primary factor responsible for draining the induced heat in the treated volume $[251,254,284,300]$. In many cases it is very diffuclt to implement these models because they require extenssive knowledge of the blood flow pattern and the vascular anatomy in addition to specific information about the countercurrent vessels' anatomy and their blood flow velocities and orientations. Thus, the ease in applying and modifying bioheat transfer equation along with very good results make Pennes' equation the common technique used to simulate hyperthermia.

\section{1. 1 Specific Absorption Rate (SAR):}

Usually, the absorption power factor (SAR) is used to indicate how much of the energy applied in a specific zone absorbed by tissues, hence, to estimate the temperature increase in the target zone with some considerations $[49,122-124,128]$. The high absorption rate of the applied energy is not definitely correlated to high temperature increase in local hyperthermia due to blood perfusion. The SAR factor gets averaged over any $10 \mathrm{~g}$ of body tissues in such localized thermal therapy. This is not an accurate assessment of the induced temperature, yet it is applicable in localized hyperthermia therapy.

Kok et al. have shown an analytical technique aimed at estimating the depth of a treatment during interstitial or intracavitary hyperthermia through estimating the radial heat flow [87]. The researchers attempted to limit the number of thermometers by referring the temperature distribution to the measured temperature at the surface of the 
applicator, which is the only temperature measurements that can be obtained noninvasively. The researchers found less than $10 \%$ difference between the induced and the calculated maximum temperature and offset in order of tenths of a millimetre in its location. This analytical technique is only applicable at a small distance from the applicator in range of $4 \mathrm{~mm}$. After considering the cylindrical coordinates of equation number (2) in Chapter 7 and applying boundary conditions to heat transfer coefficient; the heat transfer coefficient of the water used for their cooling system $\left(H_{a}\right)$; and the heat transfer coefficient to inhomogeneity's neighbouring tissues $\left(H_{b}\right)$; the temperature distribution $T(r)$ at radial distance $r$ away from the applicator is:

$$
\begin{gathered}
\rho_{t} \times c_{t} \times \frac{\partial T}{\partial t}=K_{t}\left(\frac{\partial^{2} T}{\partial r^{2}}+\frac{1}{r} \frac{\partial T}{\partial r}+\frac{1}{r^{2}} \frac{\partial^{2} T}{\partial \theta^{2}}+\frac{\partial^{2} T}{\partial z^{2}}\right)-c_{b} W_{b}\left(T-T_{a r t}\right)+S A R \\
K_{t} \frac{\partial T}{\partial n}+H_{a}\left(T(a)-T_{\text {water }}\right)=0 \quad \text { when } \mathrm{r}=\mathrm{a} \\
K_{t} \frac{\partial T}{\partial n}+H_{b}\left(T(b)-T_{\text {art }}\right)=0 \quad \text { when } \mathrm{r}=\mathrm{b}
\end{gathered}
$$

where the power $P$ or the $S A R$ is heat flow out of the applicator;

$$
p(r)=\frac{a}{r} p_{0} \exp \left(-\frac{(r-a)}{\mu}\right)
$$

Thus, the temperature distribution $T(r)$ at radial distance $r$ away from the applicator is:

$$
\begin{gathered}
T(r)=T(a)+a \frac{H_{a}}{K_{t}}\left(T(a)-T_{w a t e r}\right) \ln (r / a)-\frac{a \mu P_{0}}{K_{t}} \ln (r / a)+\frac{a \mu P_{0} e^{a / \mu}}{K_{t}} \ln (r / a)+ \\
\frac{a \mu P_{0} e^{a / \mu}}{K_{t}} \ln (r / a) \sum_{n=1}^{\infty} \frac{(-r / \mu)^{n}-(-a / \mu)^{n}}{n \cdot n !}
\end{gathered}
$$

where $\mu$ equals to:

$$
\mu=d\left(\ln \left(\frac{a}{a+d}\right)+1\right)^{-1}
$$


Equation number (1) is established based on the fact that there is no previous clinical knowledge of the heat transfer coefficient $H_{b}$. The transfer coefficient $H_{b}$ has been related to the applicator surface measured temperature $T(a)$ by equation (1. a). Thus, the researchers find $H_{b}$ by solving the above equation in $T(a)$ to identify the radial heat flow as a function of the radial distance $T(r)$ based upon the assumption that there is no blood flow. After that the researchers used the obtained parameter to evaluate the validity of their assumption by estimating values for the volumetric perfusion rates $W_{b}$. The estimated values are parallel to relative clinical values of 0 to $10 \mathrm{Kg} \mathrm{m}^{-3} \mathrm{~s}^{-1}$. Hence, Kok et al. have proven that the heat flow up to limited distance (in order of $\mathbf{4} \mathbf{~ m m}$ ) from the heating device can be estimated simply by knowing the applicator surface temperature. Temperature distribution and the heat transfer rate within a specific time are the element of a very large field called the thermal dosimetry. Clinical thermal doses which have been designed to provide a therapeutic protocol of clinical hyperthermia are currently out of scope of the current work in this thesis, some details can be found in references $1,19,87$, and 122 to 128 .

\section{2. Heat transfer by conduction:}

Conduction heat transfer is usually portrayed by the temperature distribution within a target volume [317-321]. Once the temperature distribution is measured, the heat transfer rates between any points within the volume can be easily determined. In a conduction heat transfer problem, Fourier's law is usually applied to find the heat transfer rate. Fourier's law is described as the following:

$$
q_{x}=-k \frac{\partial T}{\partial x}
$$


where $q_{x}$ is the heat flux in the $\mathrm{x}$-direction and $k$ is the thermal conductivity. The negative sign indicates that heat is being transported in the direction of decreasing temperature. Thus, the thermal conductivity describes the ease of heat flow in the volume.

Then the principle of conservation of energy controls the temperature distribution in the volume [317-321]. Material properties including state of matter, density, and specific heat are very important factors in specifying the amount of energy generated at each point within the volume. In solid material, for example, heat transfers by molecular activity "exchanging the kinetic and vibrational energies between neighbouring molecules". Here, materials with high-density $\rho$ and specific heat $c$ require more energy to change the temperature by one degree. 


\section{Appendix}

\subsection{Journal and peer reviewed conference publications:}

S. A. Alqarni, William Willmore, J. Albert, and C. W. Smelser, "Self-monitored and optically powered fiber optic device for localized hyperthermia and controlled cell death in vitro," the journal of bio photonics, 2018 (in the submission process)

S. A. Alqarni, J. Habel, M. Bernier, J. Albert and C. W. Smelser," Long-term Annealing Study of Ultrafast Laser Induced Regenerated and Third Order Fiber Bragg Gratings," Applied Physics 2018 (in the submission process)

S. A. Alqarni, J. Albert, and C. W. Smelser, "Fiber-grating-based Hyperthermal Therapeutic Device for mm-sized ex vivo Lethal Volume," in Advanced Photonics 2018 (BGPP, IPR, NP, NOMA, Sensors, Networks, SPPCom, SOF), OSA Technical Digest (online) (Optical Society of America, 2018), paper SeM2E.4.

S. A. Alqarni, M. Bernier, and C. W. Smelser," Annealing of High-Temperature Stable Hydrogen Loaded Fiber Bragg Gratings,IEEE Photonics Technol. Lett. 2016, 28, 939-942.

S. A. Alqarni, J. Habel, M. Bernier, and C. W. Smelser, "Comparison of first and third-order high temperature stable $\mathrm{H} 2$ loaded ultrafast induce FBG's," in Photonics and Fiber Technology 2016 (ACOFT, BGPP, NP), OSA Technical Digest (online) (Optical Society of America, 2016), paper BTh3B.

Abbas AS, Alqarni S, Shokouhi BB, Yavuz M, and Cui B, "Water soluble and metalcontaining electron beam resist poly(sodium 4-styrenesulfonate)", Mater. Res. Express, 1, 045102 (2014).

Hailu D. M., Alqarni S, Cui B and Saeedkia D, "Terahertz Surface Plasmon Resonance Sensor And Bull's Eye Structure For Material Sensing," The International Conference on Infrared, Millimeter, and Terahertz Waves (IRMMW-THz), Mainz, Germany, September 2013.

\subsubsection{Conference publications:}

A. S. Abbas, S. Alqarni, B. B. Shokouhi, A. S. Abbas, M. Yavuz and B. Cui, "Metal and organic nanostructure fabrication by electron beam lithography and dry liftoff," 14th IEEE International Conference on Nanotechnology, Toronto, ON, 2014, pp. 392-395.

Daniel M. Hailu, Sondos Alqarni, Bo Cui, Daryoosh Saeedkia, "Terahertz surface plasmon resonance sensor for material sensing," Proc. SPIE 8915, Photonics North 2013, 89151G.

\subsubsection{Conference presentations:}

Sondos A. Alqarni, Jacques Albert, and Christopher W. Smelser," Fiber-grating-based Hyperthermal Therapeutic Device for mm-sized ex vivo Lethal Volume", Biomedical Sensors I, SeM2E.4, Advanced Photonics Congress 2018, Zurich, Switzerland. (Oral Presentation) 
S. A. Alqarni, J. Albert, and C. W. Smelser, "Fiber-based Hyperthermal Therapeutic Device: ex vivo studied and mathematical modelling", POSTER-19, in the Photonics North 2018 Conference, Montréal, QC, Canada. (Poster)

S. A. Alqarni, J. Albert, and C. W. Smelser, "Thermal Modeling of the Lethal Localized Volume Induced by a Fiber-based Hyperthermal Therapeutic Device", Biophotonics and Sensors - Bloc 2, in the Photonics North 2017 Conference, Ottawa, ON, Canada. (Oral Presentation)

S. A. Alqarni, J. Habel, M. Bernier, J. Albert and C. W. Smelser, "Comparison of the Thermal Stability of Ultraviolet Regenerated and Third-Order Fiber Bragg Gratings", BIOPHOTONICS-05, in the Photonics North 2017 Conference, Ottawa, ON, Canada. (Poster)

Sondos A. Alqarni, Jacques Albert, and Christopher W. Smelser," Dual-Purpose Tilted Fiber Bragg Grating for Simultaneous Inducement of Localised Hyperthermia and Temperature Profiling, in life sciences day 2017 at Carleton University, Ottawa, ON, Canada. (Poster)

Joé Habel, Sondos Alqarni, Christopher W. Smelser and Martin Bernier, "High temperature stable type I femtosecond fiber Bragg gratings, in the Photonics North 2016 Conference.

(Oral Presentation)

Sondos A. Alqarni, Hubert Jean-Ruel, Jacques Albert, and Christopher W. Smelser,'DualPurpose Tilted Fiber Bragg Grating for Simultaneous Inducement of Localised Hyperthermia and Temperature Profiling," in the Photonics North 2016 Conference. (Oral Presentation)

Sondos A. Alqarni, Joé Habel, Martin Bernier, and Christopher W. Smelser" Comparison of first and third-order high temperature stable $\mathrm{H}_{2}$ loaded ultrafast induce FBG's, in the BGPP 2016 Conference. (Oral Presentation)

Sondos A. Alqarni, Martin Bernier, and Christopher W. Smelser," Annealing of HighTemperature Stable HydrogenLoaded Fiber Bragg Gratings", in the Photonics North 2015 Conference. (Poster)

\subsection{Awards}

The Best Student Paper Award (first place) at Photonics North 2016. May 2016 - Distinction Award from the Saudi Cultural Bureau in Canada July 2016 - Distinction Award from the Saudi Cultural Bureau in Canada March 2015 - Distinction Award from the Saudi Cultural Bureau in Canada March 2013 - Distinction Award from the Saudi Cultural Bureau in Canada 


\section{Bibliography}

1. Pang C. L. K," Hyperthermia in Oncology," translated by Lee K, CRC press, Taylor and Francis group, 2016.

2. Wang S. S," Scientists Study Whether Hyperthermia Treatment Makes Chemo More Effective," http:/www.wsj.com/articles/scientists-study-whether-hyperthermia-treatment-makes-chemomore-effective-1450117462, 2015.

3. Szasz A, et al. "Hyperthermia Results and Challenges," in Oncothermia: Principles and Practices, London, New York: Springer, pp. 17-88, 2011.

4. Habash W. R, et all," Thermal Therapy, Part 2: Hyperthermia Techniques, Critical Reviews in Biomedical Engineering, Vol. 34, pp. 491-542, 2006.

5. Baronzio G. F,'Hyperthermia In Cancer Treatment: A Primer,' Landes Bioscience and Spring Science+Business Media, LLC, 2006.

6. Bower K. S, et al.," Overview of Refractive Surgery," American Family Physician, Vol. 64, pp. 1183-1190, 2001.

7. Saal J. A, et al.," Intradiscal Electrothermal Therapy for the Treatment of Chronic Discogenic Low Back Pain," Clinical Sports Medicine, Vol. 21, pp. 167-187, 2002.

8. Zlotta A.R, et al," Minimally Invasive Therapies for Benign Prostatic Hyperplasia in the New Millennium: Long-Term Data, Current Opinions in Urology, Vol. 12, pp. 7-14, 2002.

9. Larson T. R," Rationale and Assessment of Minimally Invasive Approaches to Benign Prostatic Hyperplasia Therapy," Urology, Vol. 59, pp.12-16, 2002

10. Ruiz-Esparza J. et al.," The Medical Face Lift: a Non-invasive Nonsurgical Approach to Tissue Tightening in Facial Skin Using Nonablative Radiofrequency," Dermatology \& Surgery, Vol. 29, pp. 325-332, 2003.

11. Goldberg D. J," Lasers for Facial Rejuvenation,” American Journal of Clinical Dermatology, Vol. 4, pp. 325-332, 2003.

12. Noonan T. J, et al," Laser-Assisted Thermal Capsulorrhaphy," Arthroscopy, Vol. 19, PP. 815-819, 2003.

13. Enad J. G, et al," Electrothermal Capsulorrhaphy in Glenohumeral Instability Without Bankart Tear," Arthroscopy, Vol. 19, pp. 740-745, 2003.

14. Ito F. et al.," Immune Adjuvant Activity of Pre-Resectional Radiofrequency Ablation Protects against Local and Systemic Recurrence in Aggressive Murine Colorectal Cancer," Plos One, DOI: 10.1371, pp.1-23, 2015.

15. Chicheł A, et al," Hyperthermia - description of a method and a review of clinical applications," Review Paper, Rep Pract Oncol Radiother, Vol. 12, pp. 267-275, 2007.

16. Katrina F, et al," Thermal ablation of tumours: biological mechanisms and advances in therapy," Nature Reviews Cancer, Vol. 14, pp. 199-208, 2014.

17. Diederich J. C, Thermal Ablation and High-Tempreture Thermal Therapy: Overview of Technology and Clinical Implementation," INT. J. Hyperthermia, Vol. 21, pp. 745-753, 2005.

18. Ahmed K. et al," Hyperthermia: an effective strategy to induce apoptosis in cancer cells," Apoptosis, Vol. 20, pp. 1411-1419, 2015.

19. Rao W, et al." A Review of Hyperthermia Combined With Radiotherapy/Chemotherapy on Malignant Tumors," Critical Review in Biomedical Engineering, vol. 38, pp. 101-116, 2010.

20. Wust P, et al." Hyperthermia in combined treatment of cancer," The Lancet Oncologyhttp://oncology.thelancet.com-, vol. 3, pp.487-497, 2002.

21. Wiitjes A. J, et al," Intravesical Hyperthermia and Mitomycin-C for Carcinoma in Situ of the Urinary Bladder: Experience of the European Synergo Working Party," World J Urol, Vol. 27, pp. 319-324, 2009.

22. Kim Y-P, et al," Conventional cancer treatment alone or with regional hyperthermia for pain relief in lung cancer: A case — control study," Complementary Therapies in Medical, Vol. 23, pp. 381$387,2015$.

23. Habib C, et al," Hyperthermia in Oncology," The Journal of IHP, www.ihpmagazine.com, pp. 4952, 2012.

24. Myerson J. R, et al.," Components of a hyperthermia clinic: Recommendations for staffing, equipment, and treatment monitoring," Review article, International Journal of Hyperthermia, vol. 30, pp. 1-5, 2014. 
25. Luyen H, et al," High Frequency Microwave Ablation for Targeted Minimally Invasive Cancer Treatment," The 8th European Conference on Antennas and Propagation, Vol.14, pp. 1478-182, 2014.

26. Roemer B. R, Engineering Aspects of Hyperthermia Therapy Annu. Rev. Biomed. Eng vol. 01. pp. 347-376,1999.

27. Saccomandi $\mathrm{P}$, et al. " Techniques for temperature monitoring during laser-induced thermotherapy: An overview,” Int J Hyperthermia, vol. 29, pp. 609-619, 2013.

28. Taffoni F, et al." Optical Fiber-Based MR-Compatible Sensors for Medical Applications: An Overview," Sensors, vol. 13, pp. 14105-14120, 2013.

29. Udd E, et al."Chapter 16- Fiber Optic Biosensors," in Fiber Optic Sensors: An Introduction for Engineers and Scientists, Second Edition John Wiley \& Sons, 2011.

30. Strohbehn W. J et al," Hyperthermia and Cancer Therapy: A Review of Biomedical Engineering Contributions and Challenges," IEEE Transactions on Biomedical Engineering, Vol. BME-13, pp. 779-785, 1984.

31. Katzir A, et al," Infrared Fibers for Radiometer Thermomety in Hypothermia and Hyperthermia Treatment," IEEE Transaction on Biomedical Engineering, Vol. 36, pp. 634-637, 1989.

32. Tosi D, et. al," Monitoring of radiofrequency thermal ablation in liver tissue through fibre Bragg grating sensors array," Electronics Letters, 2014.

33. Nau H. W, et al," MRI-guided interstitial ultrasound thermal therapy of the prostate: A feasibility study in the canine model," Am. Assoc. Phys. Med., Vol. 32, pp. 733-743, 2005.

34. Mensel B, et al,." Laser-Induced Thermotherapy," Recent Results in Cancer Research, Vol.167, pp. 69-75, 2006.

35. Sato Y. et al, "Image Guidance of Breast Cancer Surgery Using 3-D Ultrasound Images and Augmented Reality Visualization,” IEEE Transactions on Medical Imaging, Vol. 17, pp. 681-693, 1988.

36. Arthur M. R et al, 3-D In Vitro Estimation of Temperature Using the Change in Backscattered Ultrasonic Energy," IEEE Transactions on Ultrasonics, Ferroelectrics, and Frequency Control, Vol. 57, pp. 1724-1733, 2010.

37. Cote L. G, et al.," Emerging Biomedical Sensing Technologies and Their Applications,” IEEE Sensors Journal, vol. 3, pp. 251-266, 2003.

38. Loke W-F et al," Magnetic Tracking System for Radiation Therapy," IEEE Transactions on Medical Circuits and Systems, Vol. 4, pp. 223-231, 2010.

39. Kok HP, et al," Current State of the Art of Regional Hyperthermia Treatment Planning: A Review," Radiation Oncology, Vol. 10, pp. 1-14, 2015.

40. Rebecca L, et al," Cancer Statistics," CA CANCER J CLIN, Vol. 68, pp.7-30, 2018.

41. Shenfeld O, et al.," Silver Halide Fiber Optic Radiometry for Temperature Monitoring and Control of Tissues Heated by Microwave," Optical Engineering, Vol. 32, pp. 216-221, 1993.

42. Corr J. S et al," A New Imaging Platform for Visualizing Biological Effects of Non-Invasive Radiofrequency Electric-Field Cancer Hyperthermia,'Plos One, DOI:10.1371, pp. 1-16, 2015.

43. Ihara I, et al," Ultrasonic Thermometry for Temperature Profiling of Heated Materials," in Advancement in Sensing Technology, Eds. Mukhopadhyay S. C et al, pp. 211-236, SpringerVerlag Berlin Heidelberg 2013.

44. Mukhopadhyay Ch. S," Wearable Sensors for Human Activity Monitoring: A Revie," IEEE Sensors Journal, vol. 15, pp. 1321-1330, 2015.

45. Polito D., et. Al," A Needlelike Probe for Temperature Monitoring During Laser Ablation Based on Fiber Bragg Grating: Manufacturing and Characterization," Journal of Medical Devices, Vol. 9, 2015.

46. Wang P et al., "Chapter 2. Basics of Sensors and Measurement," in Biomedical Sensors and Measurement , Zhejiang University Press, Hangzhou and Springer-Verlag Berlin Heidelberg 2011.

47. Tosi D, et al.," Fiber-Optic Temperature and Pressure Sensors Applied to Radiofrequency Thermal Ablation in Liver Phantom: Methodology and Experimental Measurements," Journal of Sensors, vol. 2015, pp. 1-22, 2014.

48. Samset E et al," Temperature Measurement in Soft Tissue Using a Distributed Fiber BraggGratings Sensor System,” Min Invas Ther \& Allied Technol, Vol. 10, pp. 89-93, 2001. 
49. Sapareto A. S., et al," Thermal Dose Determination in Cancer Therapy,” Int. J. Radiation Oncology Biol. Phys., Vol. 10, pp. 787-800, 1984.

50. Li Z, et al.," Interstitial Photoacoustic Sensor for the Measurement of Tissue Temperature during Interstitial Laser Phototherapy," Sensors, vol. 15, pp. 5583-5593, 2015.

51. Cravioto-Villanueva A, et al," Cytoreductive surgery with hyperthermic intraperitoneal chemotherapy (HIPEC) delivered via a modified perfusion system for peritoneal carcinomatosis of colorectal origin," Surg Today, Vol. 46, pp. 979-984, 2016.

52. Utsav M," Literature Survey on Carbon Nanotubes and Their Potential Applications in Cancer Treatment," Proceedings of the 14th IEEE International Conference on Nanotechnology, pp. 678-681, 2014.

53. Sazgarnia A. et al," Investigation of Thermal Distribution for Pulsed Laser Radiation in Cancer Treatment with Nanoparticle-Mediated Hyperthermia," Journal of Thermal Biology, Vol. 47, pp. 32-41, 2015.

54. Xie B. et al," Heat Localization for Targeted Tumor Treatment with Nanoscale Near-Infrared Radiation Absorbers," Phys Med Biol, Vol. 57, pp. 5765-5775, 2012.

55. Nabil M. et al," Modelling Mass and Heat Transfer in Nano-Based Cancer Hyperthermia," Royal Society Open Science, Vol. 2, pp. 1-17, 2015.

56. Chatterjee K. D, et al," Nanoparticle-mediated hyperthermia in cancer therapy," Ther Deliv., Vol.2, pp. 1001-1014, 2011.

57. Zhang Z. J," Biomedical Applications of Shape-Controlled Plasmonic Nanostructures: A Case Study of Hollow Gold Nanospheres for Photothermal Ablation Therapy of Cancer," J. Phys. Chem. Lett. Vol. 1, pp. 686-695, 2010.

58. Kim TH. et al," Size-dependent cellular toxicity of silver nanoparticles," J Biomed Mater Res., Vol. 100, pp. 1033-43, 2012.

59. Lu W. et al," Targeted Photothermal Ablation of Murine Melanomas with Melanocyte-Stimulating Hormone Analog ^ Conjugated Hollow Gold Nanospheres "Clin Cancer Res, Vol. 15, pp. 876886, 2009.

60. Cheng FY. et al," Comparative efficiencies of photothermal destruction of malignant cells using antibody-coated silica-Au nanoshells, hollow Au/Ag nanospheres and Au nanorods," Nanotechnology., Vol 20, pp.1-9, 2009.

61. Thompson E, et al," Differential response of MCF7, MDA-MB-231, and MCF 10A cells to hyperthermia, silver nanoparticles and silver nanoparticle-induced photothermal therapy,"Int J Hyperthermia, vol. 30, pp. 312-323, 2014.

62. Burke A, et al," Long-term survival following a single treatment of kidney tumors with multiwalled carbon nanotubes and near-infrared radiation," www.pnas.org cgi doi 10.1073 pnas.0905195106, Vol. 106, pp. 12897-12902, 2009.

63. Fisher W. J. et al," Photothermal Response of Human and Murine Cancer Cells to Multiwalled Carbon Nanotubes after Laser Irradiation," Cancer Res, Vol. 70, pp. 9855-9864, 2010.

64. Wang M. et al," An Overview of Cancer Treatment by Terahertz Radiation," IEEE MTT-S International Microwave Workshop Series on RF and Wireless Technologies for Biomedical and Healthcare Applications (IMWS-BIO), 2013.

65. Wang R et al," Fabrication of high-temperature tilted fiber Bragg gratings using a femtosecond laser," Optics Express, Vol. 25, pp. 23684-23689, 2017.

66. Mihailov S. J. et al.", "Induced Bragg gratings in optical fibers and waveguides using ultrafast infrared laser and a phase mask," Laser Chem.,Vol. 2008, pp. 1-20, 2008.

67. Cotillard R. et al," Regeneration of tilted fiber Bragg gratings," Proc. of SPIE, Vol. 9157, pp. 91572S-1 to 91572S-4, 2014.

68. Ertorer E et al," Femtosecond laser filaments for rapid and flexible writing of fiber Bragg grating," Optics Express, Vol. 26, pp. 9323-9331, 2018.

69. Barrera D. et al," Tilted fiber Bragg gratings in multicore optical fibers for optical sensing," Optics Letters, Vol. 42, pp. 1460-1463, 2017.

70. Smelser C, et al. "High-Reflectivity Thermally Stable Ultrafast Induced Fiber Bragg Gratings in H2 -Loaded SMF-28 Fiber," IEEE Photonics Technology Letters, vol. 21, pp. 682-684, 2009.

71. Diederich J. C, et al," Ultrasound Applicators with Integrated Catheter-Cooling for Interstitial Hyperthermia: Theory and Preliminary Experiments," ," INT. J. Hyperthermia, Vol. 12, pp. 279297, 1996. 
72. Pearce A. J et al, "Chapter 11; Temperature Measurements," in Optical-Thermal Response of Laser-Irradiated Tissue, 2nd ed, written by Welch J. A et al, DOI 10.1007/978-90-481-8831-4-11, pp. 399-453, 2011.

73. Nau H. W. et al.," Directional Power Deposistion From Direct-Coupled and Catheter-Cooled Interstiitial UltraSound Applicators," INT. J. Hyperthermia, Vol. 16, pp. 129-144, 2000.

74. Chaichanyut M, et al," Experimental Studies on Power Control Microwave Ablation in Vitro Animal Tissues with Microwave Percutaneous Coagulator," Asia-Pacific Microwave Conference Proceedings, pp. 830-832, 2013.

75. Freudenberg S, et al., "Local application of hyperthermia in the esophagus with a heatable malleable thermoplastic stent," Eur Surg Res, Vol. 38, PP. 42-27, 2006.

76. Bowman R. R," A Probe for Measuring Temperature in Radio-Frequency-Heated Material, IEEE Microwave Theory and Techniques Socity, vol. 24, pp. 43-45, 1976.

77. Rao J. Y," In-Fiber Bragg-Grating Temperature Sensor System for Medical Applications," Journal of LightWave Technology, vol. 15. pp. 779-785, 1997.

78. Rao J. Y," Recent progress in applications of fibre Bragg grating sensors,"Optics and Lasers in Engineering, vol. 31, pp.297-324, 1999.

79. Christensen A. D," Thermal Dosimetry and Temperature Measurements," American Association for Cancer Research, vol. 39, pp. 2325-2327, 1979.

80. Vaguine A. V., et al," Multiple Sensor Optical Thermometry System for Application in Clinical Hyperthermia," IEEE Transactions on Biomedical Engineering, Vol. BME-31, pp. 168-172, 1984.

81. Ersen. A, et al, "Temperature elevation profile inside the rat brain induced by a laser beam," Journal of Biomedical Optics, vol.19, pp. 01500-1-01500-7, 2014.

82. Pinet E et al," Temperature Fiber-Optic Point Sensors: Commercial Technologies and Industrial Applications," 46th MIDEM conference, ISBN 978-961-92933-0-0, pp. 31-43, 2010.

83. Wickersheim A. K et al," Fiberoptic Thermometry and its Applications," J. Microwave Power, Vol. 22, pp. 85-94, 1987.

84. Korolyov A. V, et al," Biomedical Fiber Optic Temperature and Pressure Sensors," Biomdical Engineering, vol. 46, pp. 38-42, 2012.

85. Xianhui M. et al," Fiber Bragg Gratings Based Sensor for Measuring Temperature of in Vivo Lesion," Proc. Of SPIE, Vol. 7997, pp. 799720-1 to 6, 2011.

86. Patterson A. B et al," In Vivo Quasi-Distributed Temperature Sensing with Fiber Bragg Gratings," CLEO, PP. 402-403, 2001.

87. Kok P. H. et al," On estimation of the temperature maximum in intraluminal or intracavitary hyperthermia," Int. J. Hyperthermia, Vol. 21, pp. 287-304, 2005.

88. Rao J. Y, et al.” In-situ temperature monitoring in NMR machines with a prototype in-fibre Bragg grating sensor system," Conf Proc OSA Optical Fibre Sensors, pp. 646-9, 1997.

89. Webb J. D,et. al., "First In Vivo Trials of a Fiber Bragg Grating Based Temperature Profiling System," J. Biomed. Opt, Vol. 5, pp. 45-50, 2000.

90. Atkins CG, et al.'Instrumentation for interrogating many-element fiber Bragg grating sensors," Proc SPIE, Vol. 2444, pp. 257-266, 1995.

91. Kersey D. A, et al." Fiber-optic Bragg grating strain sensor with drift-compensated high-resolution interferometric wavelength-shift detection," Optics Letters, Vol. 18, 1993.

92. Rao J. Y, et al." Optical in-Fiber Bragg Grating Sensor Systems For Medical Applications," Journal of Biomedical Optics, vol. 3, pp. 38-44, 1998.

93. Saccomandi P, et al. " Theoretical Analysis and Experimental Evaluation of Laser Induced Interstitial Thermotherapy in Ex Vivo Porcine Pancreas,” IEEE Trans Biomed Eng, Vol.59, pp. 2958-2964, 2012.

94. Saccomandi $\mathrm{P}$, et al. "Temperature monitoring and lesion volume estimation during doubleapplicator laser-induced thermotherapy in ex vivo swine pancreas: a preliminary study," Lasers Med Sci, vol. 29, pp. 607-614, 2014.

95. Tosi D, et. al," Monitoring of radiofrequency thermal ablation in liver tissue through fiber Bragg grating sensors array," Electronics Letters, vol, 50, pp. 981-983, 2014.

96. Schneider J. C. et al," Performance Evaluation of Annular Arrays in Practice: The Measurement of Phase and Amplitude Patterns of Radio- Frequency Deep Body Applicators ," Medical Physics, Vol.22, pp. 755-765, 1995. 
97. DeWerd A. L et al, " The Phantoms of Medical and Health Physics: Devices for Research and Development," Springer New York Heidelberg Dordrecht London, 2014.

98. Minton A. J. et al," Improving the Homogeneity of Tissue-Mimicking Cryogel Phantoms for Medical Imaging," Medical Physics, Vol. 39, pp. 6796-6807, 2012.

99. Prakash P," Measurement of the Specific Heat Capacity of Liver Phantom," Physiological Measurement, Vol. 27, pp. N41-N46, 2006.

100.Datla V. N. et al," Polyacrylamide Phantom for Self-Actuating Needle-Tissue Interaction Studies," Medical Engineering and Physics, Vol. 36, pp. 140-145, 2014.

101.Leonard B. J. et al," Thermal Properties of Tissue Equivalent Phantom Materials," IEEE Transactions on Biomedical Engineering, Vol. bme-31, pp. 533-536,1984.

102. Cortela G. et al," Evaluation of Acoustic and Thermal Properties of Gellan-Gum Phantom to Mimic Biological Tissue," Pan American Health Care Exchanges (Pahce). Conference, Workshops, and Exhibits, ISBN: 978-1-4673-6968-8, 2015.

103.Bude O. R, et al," An Easily Made, Low-Cost, Tissue-Like Ultrasound Phantom Material," Journal of Clinical Ultrasound, Vol. 23, pp. 271-273, 1995.

104.Fiang C-P," Vessel Phantom Fabrication Using Rapid Prototyping Technique for Investigating Thermal Dosage Profile in HIFU Surgery," Rapid Prototyping Journal, Vol. 16, pp. 417-423.

105.Pardo D. et al," Learning Temperature Dynamics on Agar-Based Phantom Tissue Surface During Single Point CO2 Laser Exposure," Neural Process Letter, Vol. 42, pp. 55-70, 2015.

106.Dabbagh A. et al," Tissue-Mimicking Gel Phantoms for Thermal Therapy Studies," Ultrasonic Imaging, Vol. 36, pp. 291-316, 2014.

107.Lazebnik M. et al," Tissue-Mimicking Phantom Materials for Narrowband and Ultra-wide-band Microwave Applications," Physics in Medicine and Biology, Vol. 50, pp. 4245-4258, 2005.

108. Schneider C. et al," Visualization by a Matrix of Light-Emitting Diodes of Interference Effects from a Radiative Four-Applicator Hyperthermia System," International Journal of Hyperthermia," Vol. 7, pp. 355-366, 1991.

109.Choi J. M. et al," A Tissue Mimicking Polyacrylamide Hydrogel Phantom for Visualizing Thermal Lesions Generated by High Intensity Focused Ultrasound," Ultrasound in Medicine and Biology, Vol. 39, pp. 439-448, 2013.

110.Graham J. S. et al," Analysis of Changes in MR Properties of Tissues After Heat Treatment," Magnetic Resonance in Medicine, Vol. 42, pp. 1061-1071, 1999.

111.Takegami K. et al," Polyacrylamide gel containing egg white as new model for irradiation experiments using focused ultrasound," Ultrasound in Medicine and Biology, Vol. 30, pp. 1419$1422,2004$.

112.Liu Y. et al," Egg White as a Blood Coagulation Surrogate," Journal Acoustical Society of America, Vol. 128. pp. 480-489, 2010.

113.Haemmerich D. et al," In Vitro Measurements of Temperature-Dependent Specific Heat of Liver Tissue," Medical Engineering and Physics, Vol. 28, pp. 194-197, 2006.

114. Haemmerich D, et al," Measurement of Temperature-Dependent Specific Heat of Biological Tissues," Physiological Measurement, Vol. 26, pp. 59-67, 2005.

115. Andrew S, et al," Evaluation of a Tissue-Mimicking Thermochromic Phantom for Radiofrequency Ablation ,"Med. Phys., Vol. 43, pp. 4304-4311, 2016.

116.Park K. S. et al,” Reusable Ultrasonic Tissue Mimicking Hydrogels Containing Nonionic SurfaceActive Agents for Visualizing Thermal Lesions ,"IEEE Transactions on Biomedical Engineering, Vol. 57, pp. 194-202, 2010.

117.Davidson H. R. S. et al," Measurement of the Thermal Conductivity of Polyacrylamide TissueEquivalent Material," International Journal of Hyperthermia, Vol. 19, pp. 551-562, 2003.

118. Wust P. et al, " Development and Testing of SAR-Visualizing Phantoms for Quality Control in RF Hyperthermia Quality Control in RF Hyperthermia, International Journal of Hyperthermia, Vol. 10, pp. 127-142, 1994.

119. Yuan Y. et al," A Heterogeneous Human Tissue Mimicking Phantom for RF Heating and MRI Thermal Monitoring Verification," Physics in Medicine and Biology, Vol. 57, pp. 2021-2037, 2012.

120.Divkovic W. G. et al," Thermal Properties and Changes of Acoustic Parameters in an Egg White Phantom During Heating and Coagulation by High Intensity Focused Ultrasound," Ultrasound in Medicine \& Biology, Vol. 33, pp. 981-986, 2007. 
121.Browne E. J, et al," Assessment of the Acoustic Properties of Common Tissue-Mimicking Test Phantoms," Ultrasound in Medicine and Biology, Vol. 29, pp. 1053-1060, 2003.

122.Rhoon C. G. et al," CEM $43^{\circ} \mathrm{C}$ Thermal Dose Thresholds: A Potential Guide for Magnetic Resonance Radiofrequency Exposure Levels?," European Society of Radiology, Vol. 23, pp. 2215-2227, 2013.

123.Dewhirst W. M. et al," Basic Principles of Thermal Dosimetry and Thermal Thresholds for Tissue Damage from Hyperthermia," International Journal of Hyperthermia, Vol. 19, pp. 267-294, 2003.

124. Yarmolenko S. P," Thresholds for Thermal Damage to Normal Tissues: An Update, International Journal of Hyperthermia, Vol 26, pp. 1-26, 2011.

125.Landry J. et al," Hyperthermia-induced Cell Death, Thermotolerance, and Heat Shock Proteins in Normal, Respiration-deficient, and Glycolysis-deficient Chinese Hamster Cells," Cancer Research, Vol. 46, pp. 324-327.

126.Deardorff L. D. et al," Axial Control of Thermal Coagulation Using a Multi-Element Interstitial Ultrasound Applicator with Internal Cooling," IEEE Transactions on Ultrasonics, Ferroelectrics, and Frequency Control, Vol. 47, pp. 170- 178, 2000.

127.Trucu D. et al," Inverse Temperature-Dependent Perfusion Coefficient Reconstruction," International Journal of Non-Linear Mechanics, Vol. 45, pp. 542-549, 2010.

128. Saito K.,"Estimation of SAR Distribution of a Tip-Split Array Applicator for Microwave Coagulation Therapy Using the Finite Element Method," IECE Trans. Electron, Vol. E84-c, pp. 948-954, 2001.

129. Maggelakis A. S. et al," Heat Transfer in Tissue Containing a Prevascular Tumor," Appl. Math Lett, Vol. 8, pp. 7-10, 1995.

130. Gupta K. P," A Numerical Study on Heat Transfer in Tissues During Hyperthermia," Mathematical and Computer Modelling, Vol. 57, pp. 1018-1037, 2013.

131.Zujie F, et al." Chapter 4 Fiber Gratings and Related Devices," in Fundamentals of Optical Fiber Sensors, Wiley Series in Microwave and Optical Engineering Somerset, US: Wiley, 2012.

132.Canning J, "Fibre gratings and devices for sensors and lasers," Laser \& Photon. Rev. 2, No. 4, Wiley, USA, 2008.

133. Rajan G," Optical Fiber Sensors: Advanced Techniques and Applications," CRC Press 2015.

134. Webb D." Chapter 17 Fiber Bragg Gratings Sensors," in Handbook of Optical Sensors, edited by Luis J. et al, CRC Press 2014.

135.Cusano A. et al," Fiber Bragg Gratings Sensors: Recent Advancements, Industrial Applications and Market Exploitation," Bentham Since Publisher, 2011.

136. Echevarria J, et al, "Fiber Bragg Gratings First-and Second Order Diffraction Wavelengths based Transducer Optimized Design," Smart Structures and Materials: Smart Sensor Technology and Measurements Systems, Proc. SPIE, Vol. 4694, pp. 168-174, 2002.

137. Osuch T. et al," Development of Fiber Bragg Gratings Technology and their Complex Structures for Sensing, Telecommunications and Microwave Photonics Applications," Bulletin of the Polish Academy of Sciences Technical Sciences, Vol. 62, pp. 627-633, 2014.

138. Roriz P. et al," Review of Fiber-Optic Pressure Sensors for Biomedical and Biomechanical Applications," Journal of Biomedical Optics, Vol. 18, pp. 050903-1- 050903-18, 2013.

139.Ponmmozhi J. et al," Smart Sensors/Actuators for Biomedical Applications: Review," Measurement, Vol. 45, pp. 1675-1688, 2012.

140. Kinet D. et al," Fiber Bragg Grating Sensors toward Structural Health Monitoring in Composite Materials: Challenges and Solutions," Sensors, Vol. 14, pp. 7394-7419, 2014.

141.Guo J. et al," Ultrasonic Imaging of Seismic Physical Models Using a Phase-Shifted Fiber Bragg Grating," Optics Express, Vol. 22, pp. 19573- 19580, 2014.

142.Quintero M. M. S. et al," A Magnetostrictive Composite-Fiber Bragg Grating Sensor," Sensors, Vol. 10, pp. 8119-8128, 2010.

143.Lamberti A. et al," Dynamic Strain Measurements on Automotive and Aeronautic Composite Components by Means of Embedded Fiber Bragg Grating Sensors," Sensors, Vol. 15, pp. 27174$27200,2015$.

144. Sridevi S. et al," Optical Bio-Sensing Devices Based on Etched Fiber Bragg Gratings Coated with Carbon Nanotubes and Graphene Oxide Along with a Specific Dendrimer," Sensors and Actuators, Vol. 195, pp. 150-155, 2014. 
145.Roriz P. et al," From Conventional Sensors to Fibre Optic Sensors for Strain and Force Measurements in Biomechanics Applications: A Review," Journal of Biomechanics, Vol. 47, pp. 1251-1261, 2014.

146.Li H. et al," Wearable Sensors in Intelligent Clothing for Measuring Human Body Temperature Based on Optical Fiber Bragg Grating," Optics Express, Vol. 20, pp. 11740- 11752, 2012.

147. Baldini F. et al," Optical Fibre Gratings as Tools for Chemical and Biochemical Sensing," Anal Bioanal Chem, Vol. 402, pp. 109-116, 2012.

148.Johnson D.," Novel Optical Fibers: Draw-tower process creates high-quality FBG arrays," http:/www.laserfocusworld.com/articles/print/volume-48/issue-10/features/novel-optical-fibersdraw-tower-process-creates-high-quality-fbg-arrays.html, 2012.

149. Wu M-C. et al," Fabrication of Extremely Short Length Fiber Bragg Gratings for Sensor Applications," Proceeding of IEEE, Vol. 1, pp. 49-55, 2002.

150.Todd D. M. et al," Bragg Grating-Based Fibre Optic Sensors in Structural Health Monitoring," Phil. Trans. R. Soc., Vol. 365, pp. 317-343, 2007.

151.Lindner. E et al, “ Post-Hydrogen Loaded Draw Tower Fiber Bragg Gratings and their Thermal Regeneration,” Applied Optics, Vol. 50, pp. 2519-2522, 2011.

152.Albert J, et al." Tilted Fiber Bragg Grating Sensors," Laser Photon. Rev., Vol. 7, pp. 83-108, 2013.

153.Erdogan T, et al.” Tilted fiber phase gratings,” J. Opt. Soc., Vol. 13, pp. 296-313, 1996.

154.Dong X, et al.” Tilted Fiber Bragg Gratings: Principle and Sensing Applications," Review Photonic Sensors, Vol. 1, pp. 6-30, 2011.

155. Shik K, et al." Transmissive Tilted Gratings for LP01 -to-LP11 Mode Coupling,” IEEE Photonics Technology Letters, Vol. 11, pp. 1286-1288, 1999.

156. Brilland L. et al," Slanted Gratings UV-Written in Photosensitive Cladding Fiber," Electronics Letters, Vol. 35, pp. 234-236, 1999.

157.Lee S. K," Mode Coupling in Tilted Planar Waveguide Gratings,"Applied Optics, Vol. 39, pp. 6144- 6149, 2000.

158.Hernaez M. et al," Optical Fiber Sensors Based on Lossy Mode Resonances," Advancement in Sensing Technology, Eds. Mukhopadhyay C. S. et al., Springer-Verlag Berlin Heidelberg 2013.

159.Bouzid A, et al." Scattering Analysis of Slanted Fiber Gratings," Applied Optics, Vol. 36, pp. 558$562,1997$.

160.Zhao Y. et al," Characteristics and Applications of Tilted Fiber Bragg Gratings," Journal of Optoelectronics and Advanced Materials, Vol. 12, pp. 2343-2354, 2010.

161.Lee S. K. et al," Transmissive Tilted Gratings for LP01 -to-LP11 Mode Coupling," IEEE Photonics Technology letters, Vol. 11, pp. 1286-1288, 1999.

162.Erdogan T. et al," Radiation-Mode Coupling Loss in Tilted Fiber Phase Gratings," Optics Letters, Vol. 20, pp. 1838-1840.

163.Li Y. et al," Volume Current Method for Analysis of Tilted Fiber Gratings," Journal of Lightwave Technology, Vol. 19, pp. 1580-1591, 2001.

164.Jauregui C. et al," Near-Field Theoretical Model of Radiation From a Uniform-Tilted Fiber-Bragg Grating," Microwave and Optical Technology Letters, Vol. 37, pp. 124- 127, 2003.

165. Walker B. R," Shaping the Radiation Field of Tilted Fiber Bragg Gratings," Journal OF Optical Society of America, Vol. 22, pp. 962-975, 2005.

166.Li Y. et al," Radiation Modes and Tilted Fiber Gratings," Journal OF Optical Society of America, Vol. 23, pp. 1544-1555, 2006.

167.Erdogan T," Cladding-Mode Resonances in Short- and Long- Period Fiber Grating Filters," Journal of Optical Society of America, Vol. 14, pp. 1760- 1773.

168.Lobo E. A. et al," Gain-Flattening Filter Design Using Rotationally Symmetric Crossed Gratings," Journal of Lightwave Technology, Vol. 21, pp. 2083-2088, 2003.

169. Maguis S. et al," Biofunctionalized Tilted Fiber Bragg Gratings for Label-Free Immunosensing," Optics Express, Vol. 16, pp. 19049-19062, 2008.

170.Huy P. C. M. et al," Tilted Fiber Bragg Grating Photowritten in Microstructured Optical Fiber for Improved Refractive Index Measurement," Optics Express, Vol. 14, pp. 10359- 1370, 2006.

171.Liu F. et al," Wideband-Adjustable Reflection-Suppressed Rejection Filters Using Chirped and Tilted Fiber Gratings," Optics Express, Vol. 22, pp. 24430-24438, 2014. 
172. Gao Y, at al." Demonstration of simultaneous mode conversion and demultiplexing for mode and wavelength division multiplexing systems based on tilted few-mode fiber Bragg gratings," Optics Express, Vol. 23, pp. 9959-9967, 2015.

173.Zhou K, et al," Side Detection of Strong Radiation-Mode Out-Coupling From Blazed FBGs in Single-Mode and Multimode Fibers" IEEE Photonics Technology Letters, VOL. 15, pp. 936-938, 2003.

174.Zhou K. et al," Fiber Bragg Grating Sensor Interrogation System Using a CCD Side Detection Method With Superimposed Blazed Gratings," IEEE Photonics Technology letters, Vol. 16, pp. 1549-1551, 2004.

175.Simpson G. A. et al," Optical Sensor Interrogation with a Blazed Fiber Bragg Grating and a Charge-Coupled Device Linear Array," Applied Optics, Vol. 43, pp. 33-40, 2004.

176. Cusano A. et al," Single and Multiple Phase Shifts Tilted Fiber Bragg Gratings," Research Letters in Optics, Vol. 2009, pp. 1-4, 2009.

177. Chen C. et al," The Sensitivity Characteristics of Tilted Fibre Bragg Grating Sensors with Different Cladding Thicknesses," Measurement Science and Technology, Vol. 18, pp. 3117-3122, 2007.

178.Zhou B. et al," Cladding-Mode-Recoupling-Based Tilted Fiber Bragg Grating Sensor With a Core-Diameter-Mismatched Fiber Section," IEEE Photonics Journal, Vol. 2, pp. 152-157, 2010.

179.Xie W. X, et al," Second order diffraction efficiency of Bragg gratings written within germanosilicate fibres," Optics Communications, Vol. 101, pp.85-91, 1993.

180.Hongzhi J, et al," "First- and second-order Diffraction Characteristics of Fiber Bragg Gratings," Optics Communications, Vol. 178, pp. 339-343, 2000.

181.Yang L, et al," The Effect of Radiation Coupling in Higher Order Fiber Bragg Gratings," Piers Online, Vol. 3, pp. 462-466, 2007.

182.Sun N-H, et al.” Second-Order Fiber Bragg Gratings," 14th OptoElectronics and Communications Conference, pp. 1-2, 2009.

183.Masood T," Single-Frequency 1310-nm AlGaInAs-InP Grating-Outcoupled Surface-Emitting Lasers," IEEE Photonics Technology Letters, Vol. 16, pp. 726-728, 2004.

184.Ye J. et al," Radiation Resistance of Single-Frequency 1310-nm AlGaInAs-InP GratingOutcoupled Surface-Emitting Lasers," IEEE Photonics Technology Letters, Vol. 18, pp. 148$150,2006$.

185. Mihailov S, "Fiber Bragg Grating Sensors for Harsh Environments," Sensors, Vol. 12, pp. 1898 1918, 2012.

186.Lindner E, et al. "Thermal Regeneration of Fiber Bragg Gratings in Photosensitive Fibers," Optics express, Vol. 17, pp. 12523-12531, 2009.

187. Aslund M. et al, " Annealing Properties of Gratings Written into UV-Presensitized HydrogenOutdiffused Optical Fiber," Optics Letters, Vol. 25, pp. 692-694, 2000.

188. Bandyopadhyay S. et al," Ultrahigh-Temperature Regenerated Gratings in Boron-Codoped Germanosilicate Optical Fiber Using 193 nm,” Optics Letters. Vol. 33, pp. 1917-1919, 2008.

189.Zhang B. et al, "High-Temperature Resistance Fiber Bragg Grating Temperature Sensor Fabrication," IEEE Sensors Journal, Vol. 7, pp. 586-591, 2007.

190. Yang H-Z. et al, " 1.3 and $1.55 \mu \mathrm{m}$ Thermally Regenerated Gratings in Hydrogenated Boron/Germanium Co-Doped Photosensitivity Fiber," IEEE Sensors Journal, Vol. 14, pp. 1352 1356, 2014.

191.Canning J. et al," Extreme Silica Optical Fiber Gratings," Sensors, Vol. 8, pp. 6448-6452, 2008.

192. Yang H. Z. et al," Thermal Regenerated Grating Operation at Temperatures up to $1400^{\circ} \mathrm{C}$ Using New Class of Multimaterial Glass-Based Photosensitive Fiber," Optics Letters, Vol. 39, pp. 64386441, 2014.

193.Canning J. et al," Regenerated Fibre Bragg Gratings," Frontiers in Guided Wave Optics and Optoelectronics, Available from: http://www.intechopen.com/books/frontiers-in- guided- waveoptics-and- optoelectronics/regenerated-fibre-bragg-gratings, 2010.

194.Bandyopadhyay S. et al." A study of Regenerated Gratings Produced in Germanosilicate Fibers by High Temperature Annealing," Optics Express Vol. 19, pp. 1198-1206, 2011.

195.Polz L. et al," Fiber Bragg Gratings in Hydrogen-Loaded Photosensitive Fiber with Two Regeneration Regimes,” Opt. Commun, Vol. 313, pp. 128-133, 2014. 
196.Lai H. M. et al,“ Thermal Stress Modification in Regenerated Fiber Bragg Grating via Manipulation of Glass Transition Temperature Based on CO2- Laser Annealing," Opt. Lett., Vol. 40, pp. 748-751, 2015.

197.Lim S. K. et al, "Axial contraction in etched optical fiber due to internal stress reduction," Opt. Express, Vol. 21, pp. 2551-2562, 2013.

198. Bueno A. et al," Fast thermal regeneration of fiber Bragg gratings," Opt. Lett., Vol. 38, pp. 4178-4181, 2013.

199.Coradin K. F. et al, "Long-Term Stability Decay of Standard and Regenerated Bragg Gratings Tailored for High Temperature Operation," J. Microw. Optoelectron. Electromagn. Appl, Vol. 12, pp. 719-729, 2013.

200.Celikin M. et al," Development of Regenerated Fiber Bragg Grating Sensors with Long-Term Stability," Optics Express, Vol. 24, pp. 21897-21909, 2016.

201.Hill O. K. et al," Bragg Gratings Fabricated in Monomode Photosensitive Optical Fiber by UV Exposure Through a Phase Mask," Applied Physics Letters, Vol. 62, pp. 1035-1037, 1993.

202. Melo M. et al," Fabrication of Tailored Bragg Gratings by the Phase Mask Dithering/Moving Technique," Photonic Sensors Vol. 3, pp. 81-96, 2013.

203. Statkiewwicz-Barabach G. et al," Fabrication of Multiple Bragg Gratings in Microstructured Polymer Fibers Using a Phase Mask with Several Diffraction Orders," Optics Express, Vol. 21, pp. 8521-8534, 2013.

204.Mihailov J. S. et al," Fabrication of Tilted Fibre-Grating Polarisation-Dependent Loss Equaliser," Electronics Letters, Vol. 37, pp. 284-286, 2001.

205. Voisin V. et al," Self-Referenced Photon Counting OTDR Technique for Quasi-Distributed Fiber Bragg Gratings Sensors,” IEEE Sensors Journal, Vol. 12, pp. 118- 123, 2012.

206.Xu Y. et al," Optical Fiber Random Grating-Based Multiparameter Sensor,” Optics Letters, Vol. 40, pp. 5514-5517, 2015.

207. Voorthuyzen A. J. et al," Investigations of the Surface Conductivity of Silicon Dioxide and Methods to Reduce it," Surface Science, Vol. 187, pp. 201-211, 1987.

208. Wang X. et al," Hot-Wire Anemometer Based on Silver-Coated Fiber Bragg Grating Assisted by No-Core Fiber," IEEE Photonics Technology letters," Vol. 25, pp. 2458-2461, 2013.

209.Li Ying. Et al,” Microfluidic Flowmeter Based on Micro "Hot- Wire” Sandwiched Fabry-Perot Interferometer," Optics Express, Vol. 23, pp. 9483-9493, 2015.

210.Caldas P. et al," Fibre Optic Hot-Wire Flowmeter Based on a Metallic Coated Hybrid long Period Grating/Fiber Bragg Grating Structure,” Appl Opt. Vol. 17, pp. 2738-2743, 2011.

211.https://netl.doe.gov/File\%20Library/Research/Coal/cross-cutting\%20research/CTR-Sensors-andControls-Project-Portfolio-2015.pdf

212.Bernier M, et al. "High mechanical strength fiber Bragg gratings made with infrared femtosecond pulses and a phase mask," Opt. Lett., vol. 39, pp. 3646-3649, 2014.

213. Smelser C, et al. "Formation of Type I-IR and Type II-IR gratings with an ultrafast IR laser and a phase mask," Opt. Express, vol. 13, pp. 5377-5386, 2005.

214.Cook K, et al. "Regenerated femtosecond fibre gratings," Proc. SPIE 8351, pp. 835111-1-8351116,2012 .

215. Roti Roti L. J.," Review, Cellular responses to hyperthermia (40-46 $\left.{ }^{\circ} \mathrm{C}\right)$ : Cell killing and molecular events," Int. J. Hyperthermia, Vol. 24, pp.3-15, 2008.

216.Kase R. K, et al," Comparison of some response to hyperthermia by normal human diploid cells and neoplastic cells from the same origin,” Europ. J. Cancer, Vol. 12, pp. 481-491, 1976.

217.Curley A. S, et all," The Effects of Non-Invasive Radiofrequency Treatment and Hyperthermia on Malignant and Nonmalignant Cells," Int. J. Environ. Res. Public Health, Vol. 11, pp. 9142-9153, 2014.

218. Madlener S et all," Short 428C heat shock induces phosphorylation and degradation of Cdc25A which depends on p38MAPK, Chk2 and 14.3.3,"Human Molecular Genetics, Vol. 18, pp. 19902000, 2009.

219.Fevold H," Egg Proteins," Advances in protein chemistry ,'Vol. 6, pp. 187-252, 1951.

220.Anson M. L.," Protein Denaturation and the Properties of Protein Groups," Advances in protein chemistry, Vol. 2C, pp. 361-386, 1945.

221. Mallamace F. et al," Energy landscape in protein folding and unfolding," PNAS, Vol. 113, pp. 3159-3163, 2016. 
222.Almquist J. H. et al," Determination of Total Solid Matter and Density of Egg White by Refractometer," Ind. Eng. Chem. Anal. Ed., Vol.4, pp 305-306, 1932.

223.Mine Y, et al.” Thermally Induced Changes in Egg White Proteins," J. Agric. Food Chem., Vol (38), pp. 2122-2125, 1990.

224.Abbasnezhad B. et al," Thermophysical and rheological properties of liquid egg white and yolk during thermal pasteurization of intact eggs," Food Measure, Vol.8, pp. 259-269, 2014.

225.Pham T. Q.," Food Freezing and Thawing Calculations," Springer Briefs in Food, Health, and Nutrition, 2014.

226.Ferreira M. et al," A calorimetric study of egg white proteins," Journal of Thermal Analysis, Vol. 48, pp. 683-690, 1997.

227.Kato Y. et al," Effect of the Maillard Reaction on the Attributes of Egg White Proteins," Agric. Bioi. Chem.Vol (42), pp. 2233-2237, 1978.

228. Weijers M, et al," Heat-induced denaturation and aggregation of ovalbumin at neutral $\mathrm{pH}$ described by irreversible first-order kinetics," Protein Science, Vol. 12, pp. 2693-2703, 2003.

229. Chen S. S. et al," heat-induced changes in the Mechanics of a collagenous Tissue: Isothermla Free Shrinkage, Journal of Biomechanical Engineering, Vol. 119, pp. 372-378, 1997.

230.Mine Y.," Recent advances in the understanding of egg white protein functionality," Trens in Food Science \& Technology, Vol. 6, pp. 225-232, 1995.

231.Plancken I. VD, et al,." Kinetic Study on the Changes in the Susceptibility of Egg White Proteins to Enzymatic Hydrolysis Induced by Heat and High Hydrostatic Pressure Pretreatment," J. Agric. Food Chem. Vol. 52, pp. 5621-5626, 2004.

232.Plancken I. VD, et al,.” Heat-Induced Changes in the Susceptibility of Egg White Proteins to Enzymatic Hydrolysis: a Kinetic Study,” J. Agric. Food Chem. Vol. 51, pp. 3819-3823, 2003.

233.Atılgan M. R. et al," Rheological Properties of Liquid Egg Products (LEPS),'International Journal of Food Propertie, Vol. 11, pp. 296-309, 2008.

234.Plancken I. VD, et al,." Combined effect of high pressure and temperature on selected properties of egg white proteins," Innovative Food Science and Emerging Technologies, Vol (6), pp. 11-20, 2005.

235.Zhang H. et al," An improved hot probe for measuring thermal conductivity of liquids," Meas. Sci. Technol. Vol. 16, pp. 1430-1435, 2005.

236.Punidadas P. et al," Selected physical properties of liquid egg products at pasteurization temperature," Journal of Food Processing and Preservation , Vol.23, pp. 153-168, 1998.

237.Kauzmann W," Some Factors in the Interpretation of Protein Denaturation," Advances in protein chemistry, Vol. 14, pp. 1-57, 1959.

238.Coimbra S. R. J.et al," Density, heat capacity and thermal conductivity of liquid egg products," Journal of food engineering, Vol. 74, pp. 186-190, 2006.

239.Parkinson T.L.," The effect of heat and other factors on whole egg and its constituents," Journal of the science of food and agriculture," Vol. 17, pp. 233-236, 1966.

240.Cunningham F. E. et al," Stabilization of Egg-White Proteins to Pasteurizing Temperature Above 60 C," Food technology, Vol. 19, pp. 136-141, 1965.

241.ASHRAE Handbook - Refrigeration (I-P Edition). American Society of Heating, Refrigerating and Air-Conditioning Engineers, Inc. "Chapter 9: Thermal Properties of Foods", 2006.

242.Hart L. F. et al," Modern food analysis," Springer Berlin Heidelberg 1971.

243.Huopalahti R. et al," Bioactive egg compounds," springer 2007.

244.Denys S. et all," Combined CFD and Experimental Approach for Determination of the Surface Heat Transfer Coefficient During Thermal Processing of Eggs," journal of food science, Vol. 68. pp. 943-951, 2003.

245.Matt F. C. et al," Heat Conduction in Two-Phase Composite Materials with Three-Dimensional Microstructures and Interfacial Thermal Resistance," Springer-Verlag Berlin Heidelberg :Heat Transfer in Multi-Phase Materials, pp. 63-97, 2010.

246. Michalski J. et al, An Analysis of Heat Conduction with Phase Change during the Solidification of Copper," Excerpt from the Proceedings of the COMSOL Conference 2009 Boston.

247.Abbasnezhad B. et al," Numerical modeling of heat transfer and pasteurizing value during thermal processing of intact egg," Food Science \& Nutrition published, Vol. 4, pp. 42-29, 2016.

248. Smelser C, et al. "Rouard's method modeling of type I-IR fiber Bragg gratings made using an ultrafast IR laser and a phase mask," JOSA., vol. 23, pp. 2011-2017 2006. 
249.Alqarni S, et al., "Annealing of High-Temperature Stable Hydrogen Loaded Fiber Bragg Gratings," IEEE Photonics Technology Letters, vol. 28, pp. 939-942, 2016.

250. Alqarni S, et al," Comparison of First and Third-Order High Temperature Stable H2 Loaded Ultrafast induce FBG's," in Photonics and Fiber Technology 2016 (ACOFT, BGPP, NP), OSA.

251.Huang H-W et al.," Chapter 1: Bioheat Transfer and Thermal Heating for Tumor Treatment," Becker M. S. et al," Heat Transfer and Fluid Flow in Biological Processes," Elsevier Inc, 2015.

252. Shafirstein G. et al," The role of mathematical modelling in thermal medicine," Int J Hyperthermia, Vol 29, pp. 259-261 , 2013.

253.Khanafer K. et al," Chapter 1: Synthesis of Mathematical Models Representing Bioheat Transport ," in Advances in Numerical Heat Transfer, Volume 3 by Minkowycz J. W. et al, CRC Press, 2009.

254.Kok P. H. et al," Planning optimisation and evaluation of hyperthermia treatments," International journal of hyperthermia, Vol. 33, pp. 593-607, 2017.

255. Schooneveldt G. et al," Thermal dosimetry for bladder hyperthermia treatment. An overview.," Department of Radiation Oncology Faculty Papers. Paper 87. h p://jdc.je erson.edu/radoncfp/87, 2016.

256.Datta A et al," An Introduction to Modeling of Transport Processes: Applications of Biomedical Systems," Cambridge University Press, 2010.

257.Diersch H-J," FEFLOW Finite Element Medeling of Flow Mass and Heat Transport in Porous and Fractured Media," Springer-Verlag Berlin Heidelberg, 2014.

258.Datta K. A," Biological and Bioenvironmental Heat and Mass Transfer," Boca Raton: CRC Press, 2002.

259. Rossmann Ch. Et al," Review of temperature dependence of thermal properties, dielectric properties, and perfusion of biological tissues at hyperthermic and ablation temperatures," Crit Rev Biomed Eng. Vol. 42, pp. 467-492, 2014.

260.Zhang M. et al," Determination of Thermal Conductivities of Biological Tissue Protein," 3rd International Conference on Biomedical Engineering and Informatics, pp. 1664-1667, 2010.

261.Yue K. et al," Thermal Conductivity Measurement of Anisotropic Biological Tissue In Vitro," Int J Thermophys, Vol. 38, pp. 1-12, 2017.

262.Elder S. J.," Thermocouples for Temperature Measurements on Skin Surfaces and in Living Tissue," Onderstepoort Journal of Veterinary Science and Animal Industry, Vol. 17, pp. 141-163, 1941.

263.Zhang H. et al," A dual-thermistor probe for absolute measurement of thermal diffusivity and thermal conductivity by the heat pulse method," Measurement Science and Technology, Vol. 14, pp. 1396-1401, 2003.

264. Valvano W. J. et al," Thermal Conductivity and Diffusivity of Biomaterials Measured with SelfHeated Thermistors," International Journal of Thermophysics, Vol. 6, pp. 301-311, 1985.

265. Bill A.", Studies of the Heated Thermocouple Principle for Determinations of Blood Flow in Tissues," Acta physiol. scand , Vol. 55, pp. 111-126, 1962.

266.Zhang H. et al," Approaches to extract thermal properties from dual-thermistor heat pulse experimental data," Measurement Science and Technology, Vol. 15, pp. 221-226, 2004.

267.Balasubramanniam A.T. et al," Thermal Conductivity and Thermal Diffusivity of Biomaterials: I Simultaneous measurement Technique," Journal of Biomechanicai Engineering, pp. 148-154, 1977.

268. Bhattacharya A. et al.," Temperature dependence of thermal conductivity of biological tissues", Physiological Measurement, Vol. 24, pp. 769-783, 2003.

269.Marqa M-F, et al," Focal Laser Ablation of Prostate Cancer: Numerical Simulation of Temperature and Damage Distribution," BioMedical Engineering, Vol. 10, pp. 1-12, 2011.

270.Bravo E. M. et al," Heat Transfer in Biological Tissues," J. Klapp et al., "Selected Topics of Computational and Experimental Fluid Mechanics, "Springer International Publishing Switzerland 2015.

271. Smalcerz A. et al," Impact of Electromagnetic Field upon Temperature Measurement of Induction Heated Charges," Int J Thermophys, Vol. 34, pp. 667-679, 2013.

272. Shitzer A. et al," Thermal behavior of biological tissues-A general analysis," Bltn Mathcal Biology, Vol. 38, pp. 369-386, 1976. 
273.Zhai F. et al," Comparative experiments on phantom and ex vivo liver tissue in microwave ablation," Electromagn Biol Med, Vol. 34, pp. 29-36, 2015.

274. Sarkar S. et al," Measurement of the Thermal Conductivity of Carbon Nanotube-Tissue Phantom Composites with the Hot Wire Probe Method," Annals of Biomedical Engineering, Vol. 39, pp. 1745-1758, 2011.

275. Silva R.," Properties of Amorphous Carbon," nstitution of Engineering and Technology. 2003, Online version available at: https://app.knovel.com/hotlink/toc/id:kpPAC00002/properties-

276.Lagendijk W. J. J, "The influence of bloodflow in large vessels on the temperature distribution in hyperthermia," Phys. Med. Biol. Vol. 27, pp. 17-23 1982.

277.Becker M. S. et al," Heat Transfer and Fluid Flow in Biological Processes," Elsevier Inc, 2015.

278.Charny C. K.," Mathematical models of bioheat transfer, advances in heat transfer," Academic Press, New York, Vol 22. pp 19-155 ,1992.

279.Chen M. M. et al," Microvascular Contributions in Tissue Heat Transfer," Annals N.Y. Acad. Sci., vol. 335, pp. 137-150, 1980.

280. Weinbaum S. et al," A Two Phase Theory for the Influence of Circulation on the Heat Transfer in Surface Tissue," in M. K. Wells, Editor, Advances in Bioengineering, pp. 179-182, 1979.

281. Weinbaum S. et al,"A New Simplified Equation for the Effect of Blood Flow on Local Average Tissue Temperature," J. Biomech. Engin. Vol. 107, pp. 131-139, 1985.

282. Weinbaum S. et al," Effect of Vascular Microstructure on Surface Tissue Heat Transfer: Part I: Anatomical Foundation and Model Conceptualization, J. Biomech. Engin. Vol. 106, pp. 321-330, 1984.

283.Jiji M. L. et al," Theory and Experiment for the Effect of Vascular Microstructure on Surface Tissue Heat Transfer: Part II: Model Formulation and Solution, J. Biomech. Engin. Vol. 106, pp. 331-341, 1984.

284.Chato C. J., "Heat transfer to blood vessels," J. Biomech. Eng. 102, 110-118 1980.

285. Shrivastava D. et al," A Generic Bioheat Transfer Thermal Model for a Perfused Tissue," J Biomech Eng. Vol. 131, pp. 1-12, 2009.

286.Kotte A. et al, "A description of discrete vessel segments in thermal modeling of tissues," Phys. Med. Biol. Vol. 41, pp. 865-884, 1996.

287. Grayson J. et al," Internal calorimetry- assessment of myocardial blood flow and heat production," Journal of applied physiology, Vol. 30, pp.251-257, 1971.

288. Kandala K. S. et al,” SIMULATION OF DISCRETE BLOOD VESSEL EFFECTS ON THE THERMAL SIGNATURE OF A MELANOMA LESION," Int Mech Eng Congress Expo, Vol. 3B, pp.1-16, 2014.

289. Crezee J. et al, "Temperature uniformity during hyperthermia: the impact of large vessels," Phys. Med. Biol. Vol. 37, pp. 1321-1337, 1992.

290.Kolios C. M., et al., "Blood flow cooling and ultrasonic lesion formation," Med. Phys. 23, 12871298, 1996.

291.Grangsjo G. et all," Theory of the Heated Thermocouple Principle,"Acta physiol. scand. Vol. 66, pp. 366-373, 1966.

292.Chang A. I. et al," Thermal modeling of lesion growth with radiofrequency ablation devices," BioMedical Engineering OnLine, Vol. 3, pp. 1-19, 2004.

293. Bernardi RJ. et al,'Immunonanoshells for targeted photothermal ablation in medulloblastoma and glioma: an in vitro evaluation using human cell lines," J Neurooncol, Vol. 86, pp. 165-172, 2008.

294.Pennes H. H.," Analysis of Tissue and Arterial Blood Temperatures in the Resting Human Forearm," Journal of Applied Physiology, Vol. 1, pp. 93-122, 1948.

295. Wissler H. E.," Pennes' 1948 paper revisited,” the American Physiological Society, pp. 35-41, 1998.

296. Shih T-C. et al," Numerical analysis of coupled effects of pulsatile blood flow and thermal relaxation time during thermal therapy," International journal of heat and mass transfer, Vol. 55, pp. 3763-3773, 2012.

297. Gupta K. P. et al," A numerical study on heat transfer in tissues during hyperthermia," Mathematical and Computer Modelling, Vol. 57, pp. 1018-1037, 2013.

298. Kok HP. et al,’Thermal modelling using discrete vasculature for thermal therapy: A review," Int J Hyperthermia, Vol. 29, pp. 36-45, 2013. 
299. Khachfe A. R. et al," Determination of heat sources and heat transfer coefficient for twodimensional heat flow - numerical and experimental study," International Journal of Heat and Mass Transfer, Vol. 44, pp. 1309-1322, 2001.

300.Chang W. et al," Implication of Blood Flow in Hyperthermic Treatment of Tumors," IEEE transactions on biomedical engineering, Vol. BME-31, pp.9-16, 1984.

301. Nakayama A. et al," A rigorous derivation of the bioheat equation for local tissue heat transfer based on a volume averaging theory," Heat Mass Transfer, Vol. 46, pp. 739-746, 2010.

302.Xiong G. et al," Simulation of blood flow in deformable vessels using subject- specific geometry and spatially varying wall properties," Int j numer method biomed eng. Vol. 27, pp. 1000-1016, 2011.

303. Shibib S. K. et al," The effect of laser power, blood perfusion, thermal and optical properties of human liver tissue on thermal damage in LITT," Lasers Med Sci, Vol. 32, pp. 2039-2046.

304.Guntur R. S. et al," TEMPERATURE-DEPENDENT THERMAL PROPERTIES OF EX VIVO LIVER UNDERGOING THERMAL ABLATION," Ultrasound in Medicine and Biology, Vol. 39, pp. 1771-1784, 2013.

305.Tungjitkusolmun S. et al," Three-Dimensional Finite-Element Analyses for Radio-Frequency Hepatic Tumor Ablation," IEEE Transactions on biomedical engineering, Vol. 49, pp. 3- 9, 2002.

306. Singh J. et al," Solution of fractional bioheat equations by finite difference method and HPM," Mathematical and Computer Modelling, Vol. 54, pp. 2316-2325, 2011.

307. Shen W. et al," Modeling and Numerical Simulation of Bioheat Transfer and Biomechanics in Soft Tissue," Mathematical and Computer Modelling, Vol.41, pp. 1251-1265, 2005.

308.Cuplov V. et al," Simulation of nanoparticle-mediated near-infrared thermal therapy using GATE," Biomedical optics express, Vol. 8, pp. 1665- 1681, 2017.

309.Elkayal A. H. et al," Microwaves for breast cancer treatments,"Alexandria Engineering Journal, Vol. 54, pp. 1105-1113, 2015.

310.Hassan M. O. et al," Modeling of Ultrasound Hyperthermia Treatment of Breast Tumors," 26thNational radio science conference, NRSC' 2009.

311. Khanafer K. et al," Influence of pulsatile blood flow and heating scheme on the temperature distribution during hyperthermia treatment," International Journal of Heat and Mass Transfer, Vol. 50, pp. 4883-4890, 2007.

312.Beacco C. M. et al," Development and Experimental In Vivo Validation of Mathematical Modeling of Laser Coagulation," Lasers in Surgery and Medicine, Vol. 14, pp. 362-373, 1994.

313.Zhang C. et al," Numerical Study on the Multi-Region Bio-Heat Equation to Model Magnetic Fluid Hyperthermia (MFH) Using Low Curie Temperature Nanoparticles," IEEE Transactions on Nanobioscience, Vol. 7, pp. 267-275, 2008.

314.Olayiwola O. R. et al," Modeling and Simulation of Hyperthermia as Cancer Treatment," International Journal of Science and Technology, Vol. 2, pp. 590-595, 2012.

315.Chiang J. et al," Computational Modelling of Microwave Tumour Ablations," Int J Hyperthermia, Vol. 29, pp. 308-317, 2013.

316. Horng T-L et al," Effects of pulsatile blood flow in large vessels on thermal dose distribution during thermal therapy," Med. Phys., Vol (34), pp. 1312-1320, 2007.

317.Wang L. et al," Heat Conduction: Mathematical Models an Analytical Solutions," Springer-Verlag Berlin Heidelberg, 2008.

318. Hartnett P. J. et al," Handbook of Heat Transfer. (3rd ed.)," McGraw-Hill Companies, Inc.1998.

319. Bejan A. et al," Heat Transfer Handbook, Volume 1," John Wiley \& Sons, Inc. 2003.

320.Lienhard H. J. et al," A Heat Transfer Textbook, third edition," Cambridge, Massachusetts, U.S.A. 2001.

321.Jiji M. L.,” Heat Conduction: Third Edition,” Springer-Verlag Berlin Heidelberg, 2009.

322. Smelser C. W. et al, "The impact of index change saturation on the growth behavior of Type I ultrafast induced fiber Bragg gratings," Journal of the Optical Society of America B, vol. 25, pp. 877-883, 2008.

323. S. A. Alqarni, J. Albert, and C. W. Smelser, "Fiber-grating-based Hyperthermal Therapeutic Device for mm-sized ex vivo Lethal Volume," in Advanced Photonics 2018 (BGPP, IPR, NP, NOMA, Sensors, Networks, SPPCom, SOF), OSA Technical Digest (online) (Optical Society of America, 2018), paper SeM2E.4. 
